\title{
Inequalities in the use of reproductive health care in Sub-Saharan Africa
}

Citation for published version (APA):

Ogundele, O. (2020). Inequalities in the use of reproductive health care in Sub-Saharan Africa: evidence from Ghana and Nigeria. [Doctoral Thesis, Maastricht University]. Maastricht University. https://doi.org/10.26481/dis.2020110500

Document status and date:

Published: 01/01/2020

DOI:

10.26481/dis.2020110500

Document Version:

Publisher's PDF, also known as Version of record

\section{Please check the document version of this publication:}

- A submitted manuscript is the version of the article upon submission and before peer-review. There can be important differences between the submitted version and the official published version of record.

People interested in the research are advised to contact the author for the final version of the publication, or visit the DOI to the publisher's website.

- The final author version and the galley proof are versions of the publication after peer review.

- The final published version features the final layout of the paper including the volume, issue and page numbers.

Link to publication

\footnotetext{
General rights rights.

- You may freely distribute the URL identifying the publication in the public portal. please follow below link for the End User Agreement:

www.umlib.nl/taverne-license

Take down policy

If you believe that this document breaches copyright please contact us at:

repository@maastrichtuniversity.nl

providing details and we will investigate your claim.
}

Copyright and moral rights for the publications made accessible in the public portal are retained by the authors and/or other copyright owners and it is a condition of accessing publications that users recognise and abide by the legal requirements associated with these

- Users may download and print one copy of any publication from the public portal for the purpose of private study or research.

- You may not further distribute the material or use it for any profit-making activity or commercial gain

If the publication is distributed under the terms of Article $25 \mathrm{fa}$ of the Dutch Copyright Act, indicated by the "Taverne" license above, 


\section{INEQUALITIES IN THE USE OF REPRODUCTIVE HEALTH CARE IN SUB-SAHARAN AFRICA: EVIDENCE FROM GHANA AND NIGERIA}

Oluwasegun J. Ogundele 
ISBN: 978-94-6419-044-1

Cover concept: Nuella Amonoo

Design cover and layout: Ilse Modder | www.ilsemodder.nl

Printed by: Gildeprint Enschede | www.gildeprint.nl

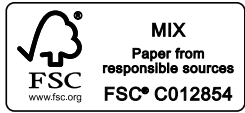

(C) Copyright 2020 Oluwasegun J. Ogundele

All rights reserved. No part of this book may be reproduced, stored in a retrieval system or transmitted in any form or by any means, without prior permission of the author. 
INEQUALITIES IN THE USE OF REPRODUCTIVE HEALTH CARE IN SUB-SAHARAN AFRICA: EVIDENCE FROM GHANA AND NIGERIA

\author{
Dissertation \\ to obtain the degree of Doctor at Maastricht University, \\ on the authority of the Rector Magnificus, \\ Prof. Dr. Rianne M. Letschert \\ in accordance with the decision of the Board of Deans, \\ to be defended in public \\ on 05 November 2020, at 10:0ohours
}

by

Oluwasegun J. Ogundele 
Supervisors:

Prof. Dr. Wim Groot

Prof. Dr. Milena Pavlova

Assessment Committee:

Prof. Dr. Hans Bosma (chairman)

Prof. Dr. Angela Brand

Prof. Dr. Franziska Gassmann

Prof. Dr. Philip Baba Adongo (University of Ghana)

Prof. Dr. Federica Angeli (University of York)

\section{Acknowledgment:}

The research presented in this dissertation was conducted at CAPHRI Care and Public Health Research Institute, Department Health Services Research of Maastricht University. CAPHRI participates in the Netherlands School of Public Health and Care Research CaRe. 


\section{CONTENTS}

List of Abbreviations $\quad 9$

Chapter 1. General Introduction $\quad 11$

Chapter 2. Inequalities in Reproductive Health Care Services across 39

Sub-Saharan Africa. A Systematic Review and Meta-Analysis

Chapter 3. Patterns of Access to Reproductive Health care services in

Ghana and Nigeria: Results of a Cluster Analysis

Chapter 4. Examining Trends in Inequality in the Use of Reproductive Health Care Services in Ghana and Nigeria

Chapter 5. Inequalities in Reproductive Health Care Use in Five West African Countries: Decomposition Analysis of the Wealth-Based Gaps in Burkina Faso, Ghana Niger, Nigeria, Senegal

Chapter 6. General Discussion

References.

Appendix. Appendix A Additional information for Chapter 2

Valorization Addendum

Acknowledgments

Curriculum Vitae

Publications 



\section{LIST OF ABBREVIATIONS}

$\begin{array}{ll}95 \% \mathrm{Cl} & 95 \% \text { Confidence Interval } \\ \text { ANC } & \text { Antenatal care } \\ \text { C-sections } & \text { Cesarean sections } \\ \text { DHS } & \text { Demographic and Health Surveys } \\ \text { EPHPP } & \text { Effective Public Health Practice Project } \\ \text { ES } & \text { Effect Size } \\ \text { GDP } & \text { Gross Domestic Product } \\ \text { GHS } & \text { Ghana Health Service } \\ \text { IMF } & \text { International Monetary Fund } \\ \text { LL } & \text { Lower Limit } \\ \text { MDGs } & \text { Millennium Development Goals } \\ \text { MPOA } & \text { Maputo Plan of Action } \\ \text { NHIS } & \text { National Health Insurance Scheme } \\ \text { PECO } & \text { Population, Exposure, Comparator, Outcome } \\ \text { PRISMA } & \text { Preferred Reporting Items for Systematic Reviews and } \\ & \text { Meta-Analyses } \\ \text { S.e. } & \text { Standard Error } \\ \text { SDGs } & \text { Sustainable Development Goals } \\ \text { SURE-P/MCH } & \text { Subsidy Reinvestment and Empowerment Programme / Maternal } \\ \text { UL } & \text { and Child Health } \\ \text { UNAIDS } & \text { Upper Limit } \\ \text { UNFPA } & \text { United Nations Programme on HIV/AIDS } \\ \text { UNICEF } & \text { United Nations Populations Fund } \\ \text { USAID } & \end{array}$





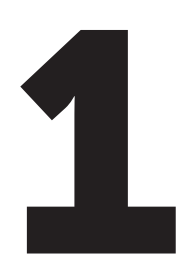

GENERAL INTRODUCTION 


\subsection{THE SCOPE OF THE DISSERTATION}

Within- and between-country inequities in health care exist irrespective of the health care system and the amount of money spent on it. Countries, including those in the Sub-Saharan African region, aim to provide equal access to health care services with adequate quality without exposing the user to financial hardship in the process (African Union, 2018; World Bank, 2016). The key actionable strategy to achieve this aim is a people-centered focus that targets the determinants of health as well as the expansion of service delivery to marginalized groups (World Bank, 2016). It is well recognized that health care provision and the distribution of the health infrastructure (supply-side factors) as well as the social determinants of health (demand-side factors) influence access (CSDH, 2008). But the question of how to assure equitable access to and use of good quality health care services lingers. Recently, countries in Sub-Saharan Africa have been experimenting with policy reforms that purportedly foster equality in access by addressing finance-related factors on the health care supply-side (African Union, 2018; Gilson \& McIntyre, 2005; Hercot, Meessen, Ridde, \& Gilson, 2011; World Bank, 2016). These reforms have included, among others, interventions to improve access to good quality reproductive health care services (African Union, 2018). There are concerns however that resources for these interventions might be depleted before reaching the marginalized groups and efforts might end up widening the disparities in health (Gilson \& Mclntyre, 2005; Rodney \& Hill, 2014). Sources of inequities as well as the interventions to address them may vary across countries. Progress on reducing inequities can be measured and monitored using inequality metrics, next to metrics such as overall utilization rates. Monitoring and evaluating interventions are valuable for both the design and implementation of health interventions. This dissertation addresses this matter by identifying socioeconomic and service-related factors that sustain barriers to the use of reproductive health care.

Reproductive health care is an essential element of the policy target to provide health care for all (male or female, adolescents, or older population) (CESCR, 2016; ICPD, 2014). Still, reproductive health care services are not always available or accessible to all, creating undesirable outcomes including reproductive tract infections, sexually transmitted diseases, unintended pregnancies, unsafe abortions, and other reproductive morbidities across population groups (CESCR, 2016; Ezeh et al., 2016; Singh-Manoux et al., 2008). Patterns of reproductive health within a population reflect country policies as well as differences in determinants of health. These factors not only influence the reproductive choices of individuals but also their ability to act on their needs for health (CESCR, 2016; CSDH, 2008). National laws and policies, as well 
as societal expectations, are central to the outcomes and distribution of reproductive health across groups. As suggested in the literature, "women are not only subject to specific inequalities but that they are also subject to pervasive forms of discrimination that are woven into the political, cultural and religious fabric of societies" (Cook, 1993, p. 77).

Reproductive health of women is important especially during the conceptive years since the health of both mother and newborn is a function of the mother's health, nutrition status, and access to health care (The Partnership for Maternal, 2011). In addition, although their life expectancy is generally higher, women have higher rates of morbidity than men and use more health care services (Bertakis, Azari, Helms, Callahan, \& Robbins, 2000; Singh-Manoux et al., 2008). This is attributed to differences in biological risks, in risks acquired through societal roles and cultural expectations as well as differences in health perceptions and experience of symptoms and illnesses, and in differential health care access (Singh-Manoux et al., 2008). Consequently, women experience multifaceted forms of inequity, gender inequality, disempowerment, violence, and inability to control their own fertility (Cook, 1993; Glasier, Gulmezoglu, Schmid, Moreno, \& Van Look, 2006). The distributions of reproductive ill-health are unfairly skewed to disfavor women. It is for this reason that this dissertation specifically focuses on the inequalities in reproductive health care access among women.

Due to the nature of the health care system and demand-side factors, the access to the health care system is not distributed according to needs, and disadvantaged groups are doing far worse (Gwatkin, Bhuiya, \& Victora, 2004). Women in the Sub-Saharan African region are further disadvantaged because, as indicated by the WHO, crucial services are performed at the lowest levels, and so far have higher indices of adverse outcomes of reproductive health (WHO, 2018). These unfavorable health outcomes are indicative of poor access (availability, adequacy, accessibility, acceptability, and affordability) to reproductive health care services and barriers to utilization (Anderson, 1973; CSDH, 2008). In response, member states of the African Union have become cosigners to declarations, including the Maputo Plan of Action (MPOA), that specifically addresses access to comprehensive sexual and reproductive health care (African Union, 2018). The MPoA policy framework is aligned with the global Sustainable Development Goals (SDGs) and includes the commitment to universal access to sexual and reproductive health care services, including family planning (SDGs 3.7) and reduce inequality within and among countries (SDGs 10) (UNDP, 2019). It is expected that these programs if successfully implemented, will significantly improve women's health. Major improvements in the utilization of reproductive health care services across individuals 
are mandatory to reach the African Union's aspirations to end preventable maternal deaths, and developmental goals set out in the SDGs.

This dissertation is motivated by the fact that commitments to improve the availability of reproductive health care services and to achieve gender and health equality, will remain fantasies if end-user access is not improved. There is a need for more knowledge on within- and cross-country inequalities in health care services. Demand-side factors, coupled with socioeconomic status, influence the use of effective reproductive health interventions, and within- and between-country health inequalities remain significant (Mellor \& Milyo, 2001). The cross-country comparison of nationally representative populations, offered in this dissertation, is to produce evidence on the socioeconomic inequities in access to reproductive health care services. Policies to narrow inequality for specific health indicators may be informed by policies in countries in which the gaps are different for user groups. This dissertation adds to our knowledge and insight on how differences in inequalities across countries reflect differences in health care systems, and to what extent they can be explained by other differences, such as socioeconomic differences. It might be useful for policies to improve the current health care service system and for them to become more equitable. Therefore, this dissertation focuses on the socioeconomic inequalities in reproductive health care as well as on the contribution of individual health determinants and wealth-based disparity in service use among women in reproductive ages.

Ghana and Nigeria are selected as case studies in this dissertation. The two countries show similarities in trends in health outcomes. At the same time, the countries' health care systems differ. For example, Ghana has established a national health insurance system, while there is no such well-established system in Nigeria (Dixon, Tenkorang, \& Luginaah, 2013). Ghana and Nigeria introduced a minimal user fee in the early 1970s, which was later abandoned in both countries due to cash crunches (Ibiwoye \& Adeleke, 2008; Nguyen, Rajkotia, \& Wang, 2011). Ghana introduced free health care for all (including free-of-charge maternal care) and the insurance scheme in the country was reported to cover $65 \%$ of the population. This reduced the private out-of-pocket health expenditure to $66 \%$ in 2010 from $80 \%$ in 2000 and increased access to health care (Odeyemi $\&$ Nixon, 2013). The same cannot be said for Nigeria. Specifically, in a comparative analysis, Odeyemi and Nixon (2013) noted that the insurance coverage in Nigeria is only $3.5 \%$ of the population and private out-of-pocket health expenditure rather increased to $95 \%$ in 2010 from 93\% in 2000. These differences can be expected to result in differences in access to reproductive health care services, which we examine in this dissertation. The two countries are also compared to other countries in West Africa. 


\subsection{KEY CONCEPTS}

To frame the dissertation and forthcoming analyses, a brief explanation of the key concepts used in the dissertation is presented below. The purpose of this is to clarify how these concepts are defined and operationalized, which is important since they are viewed differently in different studies.

\subsubsection{Equity, equity gap, and coverage gap of health care services}

Equity is often described as an ethical notion that has no fixed definition. This is because policymakers and academics have conceptualized equity to describe prevailing issues of social injustice from their own perspective. Equity in health has been used to imply the balances in the amount and quality of health of different population groups. Equity in health care has a similar notion but refers to the provision and financing of health care services.

Equity in health is used to describe the absence of systematic differences in physical and mental wellbeing, disease, or in the social determinants of health between social groups (Braveman \& Gruskin, 2003). Another related and similarly vaguely used concept is health inequality, which is defined by the WHO as "differences in health status or in the distribution of health determinants between different population groups" (WHO, 2013a). Another explanation why health inequality is used interchangeably with health disparity are the avoidable differences in health associated with belonging to a disadvantaged group, that can be reduced by policies and health care system reforms (Braveman, 2006).

Equity in health care refers to the absence of systematic differences in the use and payments for health care. Health care differs from health as the former embodies the use of health services and also the allocation, financing, and quality of health care services while the latter is an indicator of wellbeing. A widely accepted definition of equity in health care by Whitehead is "[...] equal access to available care for equal need, equal utilization for equal need, equal quality of care for all" (Whitehead, 1992, p. 222). This implies that access to health care is determined by health status and payments for health care reflect the ability to pay. Equal access to health care services is tantamount to equitable health.

Narrowing health inequalities between population groups is an important public health objective that requires monitoring through comparisons. Inequality is a useful indicator for comparisons between populations, in particular, the comparison between the 
magnitude, direction, and rate of change of health care inequalities. Two distinctions are usually made with respect to equity in health status and access to health care: absolute versus relative equality, and equality versus equity.

Absolute inequality means the unequal treatment of equals. This refers to, for example, the degree to which equals with respect to health needs have equal access to health care. The other, relative inequality, entails unequal treatment of the unequal, for example, the degree to which people with different economic status, differ with respect to their access to health care. The measurement of health care inequalities can be done in absolute or relative terms. Absolute inequality points to the systematic difference in rates or means between socioeconomic groups for a certain outcome (percentage points) while relative inequality implies the ratio of rates for a certain outcome across socioeconomic groups (percentages) (Barros \& Victora, 2013). The distinction between percentage points and percentages is essential to distinguish absolute from relative differences. Whether to use relative or absolute measures of inequality is an important choice that is made with thoughtfulness since the choice of outcome measure influences the results. Relative inequalities can lead to conclusions about large inequalities while absolute inequality measures usually result in smaller magnitudes of inequalities (Barros \& Victora, 2013; Houweling, Kunst, Huisman, \& Mackenbach, 2007).

Equality means that everyone benefits the same while equity implies that everyone benefits according to the needs. Whether referring to equity or equality, both concepts can have a horizontal aspect: when individuals/groups with equal health care needs have equal access to health care. However, equity also has a vertical aspect: when individuals/groups with different health care needs have appropriately different levels of health care access (Gulliford et al., 2002). This suggests that it is fair to provide extra support to disadvantaged groups.

The disadvantaged groups include individuals with lower socioeconomic status, measured by income or education. In order to define and assess the range of factors that characterize disadvantaged groups and influence health opportunities, the recent literature has identified group-stratifiers such as place of residence, race/ ethnicity/culture/language, occupation, education, gender/sex, religion, income and other elements of socioeconomic status (Braveman, 2003; Marmot, 2005; O'Neill et al., 2014; Solar \& Irwin, 2010). These indicators are useful in monitoring policies and interventions to reduce inequalities in health between groups within a population. Drawing from the descriptions by O'Neill et al. (2014), each of these socially stratifying 
factors can contribute to differences in health status that are avoidable, unjust, and unfair. For example, a person's residence may determine the distance to service or quality of service used, which can be remedied if the necessary infrastructure is in place and health care services with good quality are easy to reach. Race, ethnicity, culture, and language are usually intertwined and could refer to a group of individuals who have distinctive characteristics. Such groups can be at a disadvantage when it comes to health care services use due to cultural practices or language barriers when communicating with health care workers. There can be differences by gender/sex in the exposure to health hazards, discrimination in access, sex-based violence and limited or no autonomy to decide when and where to use health care. Religion could lead to inequity if it denies access to a subgroup of the population because of affiliation (or lack of it) or if the choice of health care services use is refused to the individual because of the religious doctrine. More so, when a choice is enforced for a group of people, then, it is not an individual choice. Education increases knowledge about health and preventive health. It also determines the type of employment one can obtain and the occupational choices made, which in turn is linked with differences in job-related health risks, employee benefits, employer-funded insurance systems, and income. Income and socioeconomic status influence living conditions and can lead to inequity through differences in health fostering opportunities and overall health, for example through health fostering behaviors and the likelihood to access health care services.

Analogous concepts are equity gaps and coverage gaps. To put it simply, the equity gap refers to socially unfair differences in health as well as health care inequalities across population groups (Bhutta, 2005; CSDH, 2008). More importantly, the absence of an equity gap in a population is not indicative of adequate access to care within that population. Alongside is the health care service coverage gap. Coverage in itself implies the chance of someone who needs health care actually obtaining it. Effective coverage is the chance that an individual needing health care services, acquire the possible benefit realizable from it. The coverage gap then represents an estimate of the increase in coverage desirable to achieve total/universal coverage for the service needed (Bhutta et al., 2010; Countdown Equity Analysis et al., 2008; Hosseinpoor, Victora, Bergen, Barros, \& Boerma, 2011).

It might appear that the line between the various equity concepts and health can be blurred. Notwithstanding, equity concepts can help to target action to reduce disparities when measured and compared across populations. In particular, measures of inequality have been instrumental in linking the unequal distribution of wealth to health care utilization or status. 


\subsubsection{Utilization of reproductive health care service}

Access to good quality health care services is essential to the effectiveness of the health care system and to improve health. The conceptualization of access differs between scholars and there is no unanimity on its dimensions and its determinants. From the health service perspective, access to health care has been theorized as the population's ability to obtain care (Frenk, 1985), as a link between health services provider characteristics and user characteristics (Penchansky \& Thomas, 1981), and as characteristics of health care mediating between the available services and their use (Donabedian, 1973). Aday \& Andersen (1974) framework of access to medical care conceptualizes that the health care system and the population at risk are important to be considered when providing health care services. By recognizing that resources and organization of the health care system influence access, the framework of access to medical care allows the related determinants to be studied from the population-at-risk perspective. This enables the identification of health determinants by observing the characteristics inherent to health care service delivery, as well as the characteristics of the population at risk. The framework provides a platform, which defines how factors inhibiting access to medical care can be measured through the process of entry into the health services system and derived outcome from utilization. Health care use or non-use is conceptualized as a measure of access since it reflects if the needs of the population at risk are met and it also reflects socio-cultural and economic diversity of a population (Aday, 1975; Aday \& Andersen, 1974). In recent research, relevant to the population heterogeneity are various dimensions of access including (1) acceptability, which refers to social and cultural factors mediating the populations consent to seek provided care; (2) accessibility, which refers to the geographical location and physical barriers to service and if it can be reached through a suitable means; (3) affordability, which refers to the cost and time implications for the people relevant to appropriate services; (4) availability, which refers the supply of health care services in relation to services needed; (5) adequacy, which refers to the provision of quality care services (Gulliford et al., 2002; Levesque, Harris, \& Russell, 2013). Taking these dimensions into account, utilization i.e. realized access, can be measured, rather than potential access. The service delivery aspect can be thus observed by utilization. Going by the accepted definition, health care utilization "is the quantification or description of the use of services by persons for the purpose of preventing and curing health problems, promoting maintenance of health and well-being, or obtaining information about one's health status and prognosis" (Orbell et al., 2013). The use of health care is contingent not only on supply-side factors, considering that people cannot use health care services that are unavailable or inaccessible, but also on demand-side factors that are imperative to gain access when these services are available (Aday \& Andersen, 1974; Gulliford 
et al., 2002). Supply-side factors include health care system characteristics such as the health service policy, service structure, and resources, while demand-side factors are broadly categorized as predisposing, need, and enabling factors and are mainly individual user characteristics (Andersen, 1995; Gulliford et al., 2002). Information on a demand-side indicator - utilization proportion - catalogs information about demand-side factors and can inform on supply-side factors as well. Demand-side factors provide empirical evidence of possible systematic variation between or among population groups (Ensor \& Cooper, 2004; Gulliford et al., 2002). These factors are user characteristics like residence, ethnicity, occupation, gender, religion, and education, and socioeconomic status. They influence the opportunity to use services and can be useful in understanding the existing systematic differences in accessibility/utilization (O'Neill et al., 2014). In this way, utilization can be an objective indicator of access since it captures acceptability, accessibility, affordability, availability, and adequacy of health care services.

Utilization adjusted for need can help to measure the performance of a health care system and identify the consequences for equity. The utilization of health care services reflects the relation between the determinants of health, individual user's needs and provided health services (Gulliford et al., 2002; Irwin et al., 2006; Marmot, 2005). Health care need is often defined as the requirement to use health care services to remain functional and alive, unlike demand which indicates the willingness and the ability to pay for health care. Inequity in access refers to unequal use for equal need. It also refers to unequal coverage of services, thus, lessening the opportunities of receiving health care (Shengelia, Murray, \& Adams, 2003). The advantaged group usually has better use (more than the disadvantaged groups) to health care services, which creates a coverage gap (Barros et al., 2012; Hosseinpoor et al., 2011). In other words, the coverage gap in health care is a form of disparity. It is the difference in the utilization of available health care services across groups, which produces inequalities. In this dissertation, we look at the use of health care services adjusted for needs.

\subsubsection{Reproductive health care services and maternal health}

Reproductive health is crucial to a country's population health. It is an important component of general health as it could be a precondition for social, economic and human development (Starbird, Norton, \& Marcus, 2016). Reproductive health rights are fundamental to promote reproductive health. Men and women should be able to access family planning of their choice lawfully and should be able to freely decide on the number and spacing of their children. Reproductive health rights also endeavor to assure that women have access to appropriate health care services allowing women 
to be safe before, during, and after childbirth, and to have a healthy infant (CESCR, 2016; ICPD, 2014). The reproductive health and human rights scope are broadened to combine sexual health services in the definition. Reproductive health care is "[t]he constellation of methods, techniques, and services that contribute to reproductive health and well-being through preventing and solving reproductive health problems. It also includes sexual health, the purpose of which is the enhancement of life and personal relations, and not merely counseling and care related to reproduction and sexually transmitted diseases." (ICPD, 2014, p. 59). As detailed by Stover et al. (2016), maternal (and child) health stands out as a key motivation for family planning to reduce maternal mortality (Stover, Hardee, Ganatra, Garcia Moreno, \& Horton, 2016). Other rationales mentioned by the authors are human rights and equity, sustainable development, along with population and development. Reproductive health care services are important for reproductive health.

There are various interpretations of what reproductive health care and related services for women entail. Within the literature, reproductive health care includes policies, information, attitudes, practices, interventions, and commodities vital for women during their childbearing years for fertility and fertility control, health care during pregnancy and childbirth. They are necessary for improved health outcomes (Allsworth, 2017; Kerber et al., 2007). Reproductive health care is delivered through a variety of programs and services. Clarity is provided in the continuum of care for reproductive, maternal, newborn and child health on crucial services (Kerber et al., 2007; WHO, 2011). Specific to women's health, reproductive health care services include clinical care, outpatient and outreach services, and family and community care services delivered through adolescence and pre-pregnancy, pregnancy, childbirth, and postnatal periods. These services also include contraceptive and family planning services to improve the health of the mother (and child) through the prevention of unwanted pregnancies, the timing, and spacing of birth. Antenatal care (ANC) for women during pregnancy, is necessary to improve health through preventive measures such as prompt detection and management of possible pregnancy complications. Skilled childbirth services are vital for normal or emergency childbirth. Accessibility to emergency obstetric care is essential for the mothers (and newborn) during complications. Postnatal care is imperative to identify complications among mothers (and newborns) through routine visits after birth, and to facilitate referral in high-risk situations (Kerber et al., 2007; Stover et al., 2016; WHO, 2011). 


\subsection{BACKGROUND: REPRODUCTIVE HEALTH CARE IN SUB-SAHARAN AFRICA}

Generally, reproductive health is trailing in Sub-Saharan African countries, and essential services are characterized by poor access for potential beneficiaries. There is a need for policies and leadership to be effectively focused on increasing the probability of having more services in order to steer the health care system towards more equity, quality, and efficiency. In the earlier years, the structural adjustments reforms mandated reductions in public health expenditure, or privatization of health care, and eliminated free medical services in favor of cost-recovery efforts. These acts plummeted the volume and quality of health services provided within the countries (Ekwempu, Maine, Olorukoba, Essien, \& Kisseka, 1990). The reforms also introduced user fees for health care services, as well as full or partial payments for medicines. Marking the beginning of user charges, the out-of-pocket payment system made payments for health care service use a prerequisite at the point of service delivery (Ekwempu et al., 1990; Sahn \& Bernier, 1995). User charges for health were proposed by the World Bank to address inequity in the distribution of health and inefficiency by eliminating the surplus of health facility. Shortly after the user fee introduction, the use of preventive and curative services, including maternal care, declined in Sub-Saharan African countries - the region where most of the reform was implemented (Ekwempu et al., 1990; Thomson, Kentikelenis, \& Stubbs, 2017). Reforms in Sub-Saharan African countries have so far affected the quality, quantity, and availability of health care, particularly in reproductive health care, and access to care services (Ekwempu et al., 1990; Sahn \& Bernier, 1995; Thomson et al., 2017).

In almost all of Africa, inequalities increased and worsened through the 1960s until the early 1980 s resulting in wide disparities in access to reproductive health care between income groups as well as regions (Ekwempu et al., 1990). Reports have pointed to differences in use by age, geographical location and education (UNFPA, 2016; Wang, Alva, Wang, \& Fort, 2011). This situation is yet to be significantly improved. The trend suggests that wealth-related differences in four or more ANC visits (see Figure 1.1 left graph) are smaller than differences in facility-based childbirth (see Figure 1.1 right graph). It also suggests unique contextual factors per country. A closer observation indicates that in Ghana, the richest-poorest wealth-gap in facility-based childbirth is about 70 percentage points and the gap for four or more ANC visits is about 30 percentage points. In Nigeria, the richest-poorest wealth-gap in facility-based childbirth and four or more ANC visits appears to be over 65 percentage points. On the one hand, some countries in West Africa, like Cameroun, Kenya, Senegal, follow 
the high wealth-gap in facility-based childbirth and low wealth gap in four or more ANC visits, which is a pattern observed in Ghana. Other countries follow the pattern observed in Nigeria, that is, a low wealth-gap in facility-based childbirth and high wealth gap in four or more ANC visits, while a few countries, like Benin and Ethiopia, have a higher wealth gap in four or more ANC visits (see Figure 1.1).

To monitor the performance of family planning and reproductive health program, there is demand for contraception satisfied indicator, calculated based on unmet need and contraceptive prevalence rate. The proportion of demand for contraception satisfied is the proportion of women who are currently practicing contraception, among married women of reproductive age who wish to avoid pregnancy for two years or more. It offers a summary measure of the overall effectiveness of family planning services (Fabic et al., 2015). The proportion of demand for contraception satisfied indicates a difference between the poorest and richest groups of over 40 percentage points in Burkina Faso, Cameroon, Kenya, Ethiopia, Nigeria, and Uganda (see Figure 1.2). Lower gaps (less than 15 percentage points) between the poorest-richest wealth groups in demand for contraception satisfied are observable in countries like Chad, Malawi, Sierra Leone, Zambia, and Zimbabwe (see Figure 1.2). However, taking the coverage gaps between the wealth groups within and between countries could be misleading. Closer observation shows the lower demand satisfaction in Chad where just $21 \%$ of women in the highest wealth quintiles' are able to obtain contraceptives compared to $71 \%$ of the highest wealth quintile in Malawi. Delivery of family planning services has not been sufficient to improve access to and use of contraceptives among the poor populations due to deficiencies in the health care system. Across West Africa and the developing countries at large, there is a reliance on the private sector for the provision of contraceptives and other family planning commodities (Hotchkiss, Godha, \& Do, 2011). If these services are not scaled up, they will eventually leave groups in the population behind.

The gravity of inadequate reproductive health care services is evident from the high morbidity and mortality rates. The estimates of maternal deaths reported indicate that the maternal mortality ratio in Sub-Saharan Africa region was 987 per 100,000 live births in 1990 which reduced to 546 per 100,000 live births by 2015 (WHO, 2015b). The trends of maternal deaths indicate that countries in Western Africa had higher morbidity and mortality rates between 1990 and 2010 than the rest of Sub-Saharan Africa (WHO, 2015b). The lifetime risk of maternal death in West and Central Africa alone is 1 in 27. Underneath the regional figures are stark variations across and within countries. For example, the probability that a woman of reproductive age will die eventually due to a 
maternal cause (lifetime risk of maternal death) is 1 in 48 in Burkina Faso, 74 in Ghana, 23 in Niger, 22 in Nigeria, and 61 in Senegal (WHO, 2015b). Similarly, during the past decades, inefficiencies in the delivery of services have created coverage gaps across African countries. Hosseinpoor et al. (2011) examined the coverage of family planning, maternal and newborn care, immunization, and treatment of sick children in 28 SubSaharan African countries, and showed that in West and Central Africa, the poorest to wealthiest ratio is 1.5 in Ghana and 2.4 in Nigeria. Across the region, the Gambia and Sierra Leone had the poorest to wealthiest ratio lower than Ghana, 1.3, and 1.4 respectively while Côte d'Ivoire at 2.6 had a greater ratio than Nigeria. These statistics are exacerbated by low opportunities to gain access to reproductive health care services and differences in use. The median coverage gap (the difference between the poorest and wealthiest quintiles) in skilled birth attendance was 53\% in Sub-Saharan Africa, while one or more ANC visits pointed to a coverage gap of $13 \%$ (Hosseinpoor et al., 2011).

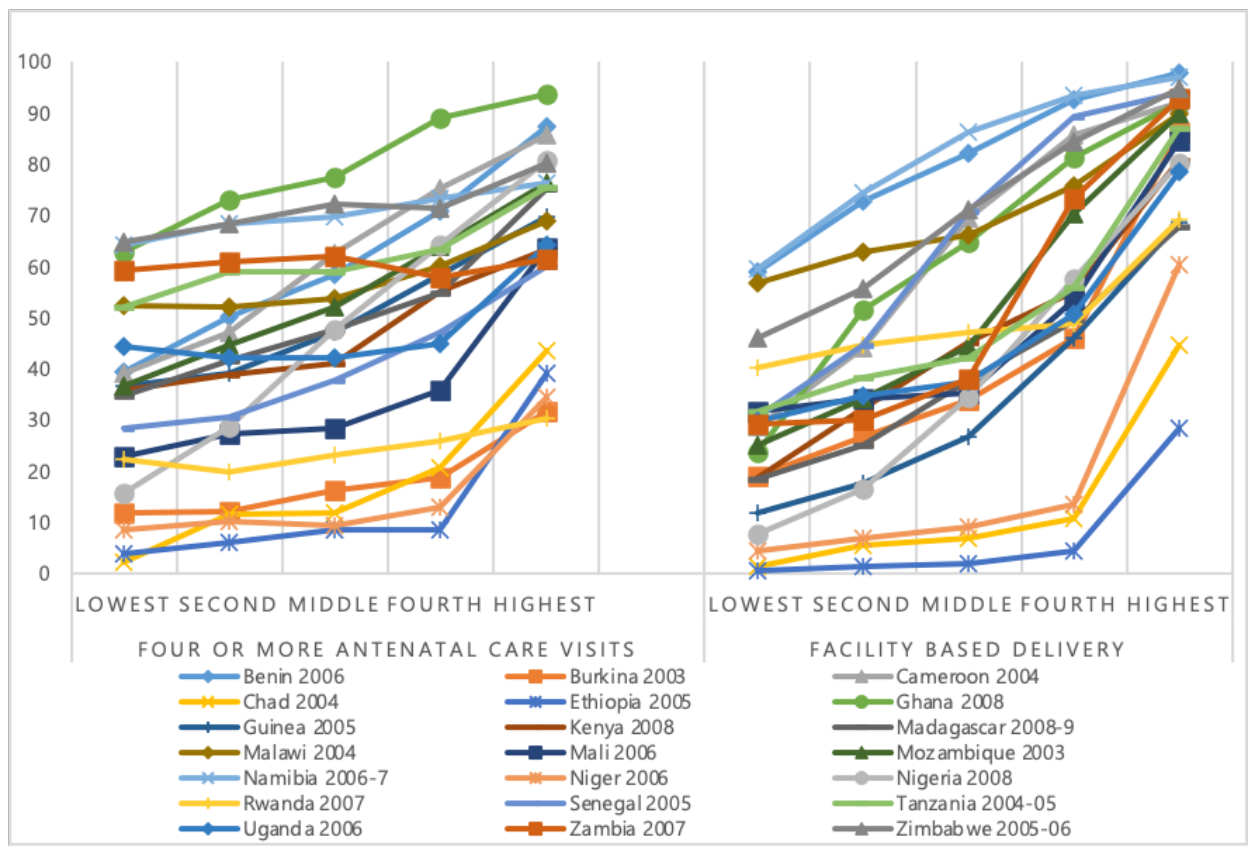

Figure 1.1: Percentage of women who received antenatal care from a skilled health provider (left), who had childbirth in a health facility (right) by wealth status, 1990-2009 (quintile). Source Wang, Alva, Wang, and Fort (2011) 


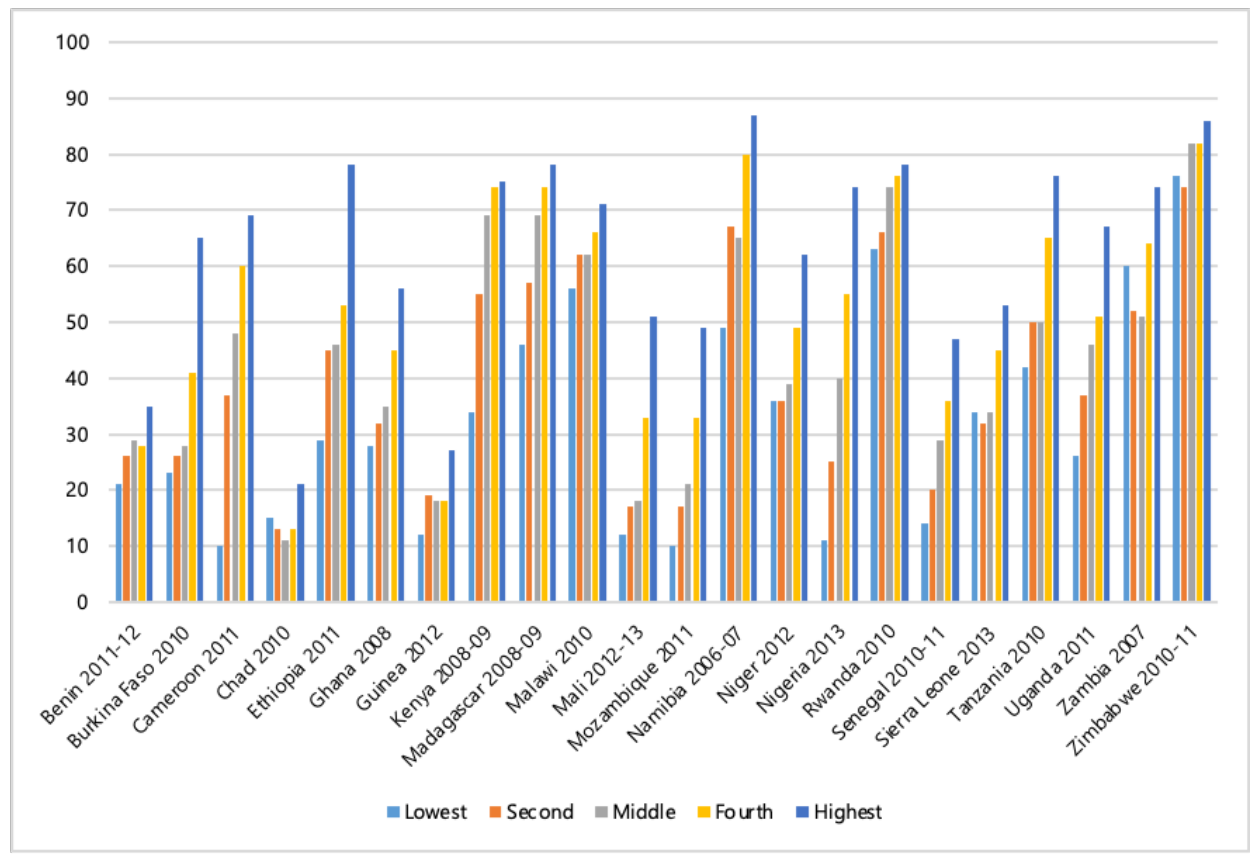

Figure 1.2: Differences in the proportion of demand for contraception satisfied among women aged 15-49, married or in a union, by wealth status (quintile). Source UNFPA (2016)

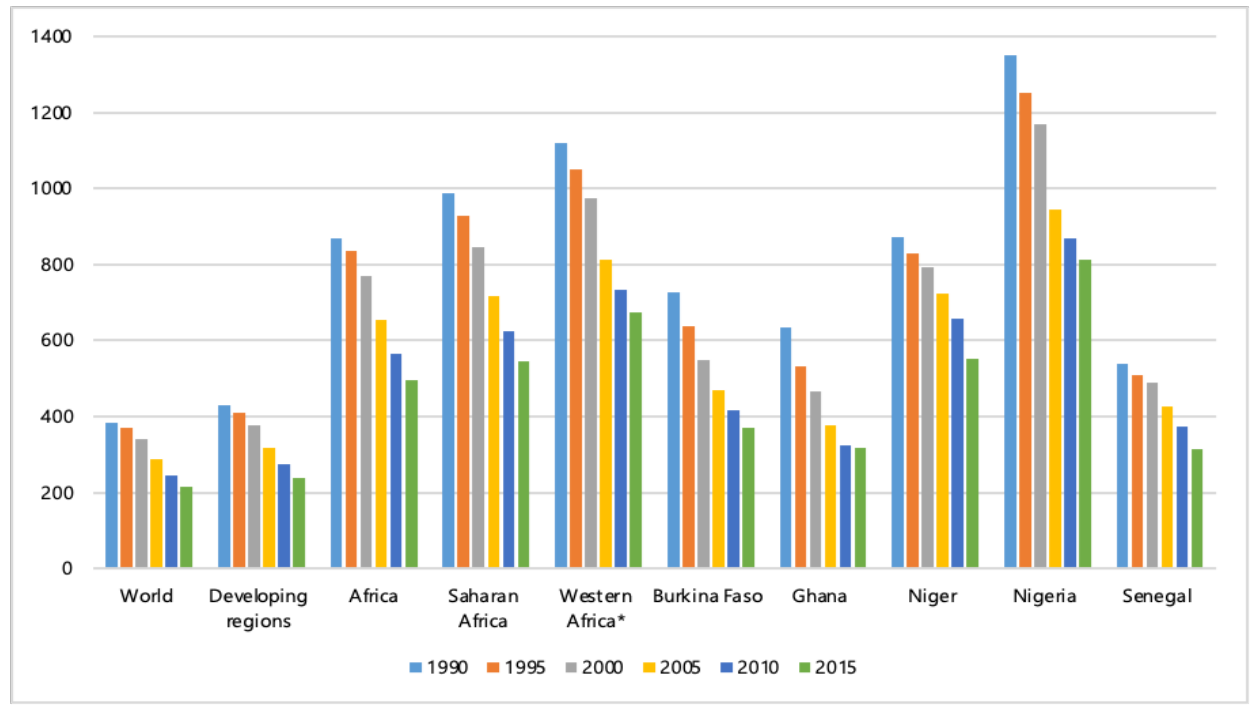

Figure 1.3: Trend and the proportion of maternal mortality in selected countries. Source WHO (2015) 
In the last two decades, countries have been deliberate in their efforts to address the barriers to reproductive health care. More recently, pro-poor programs have been introduced especially across the Sub-Saharan African region to reduce or remove user charges at point of service use following the path to universal health coverage. Despite the fact that the pro-poor initiatives, such as ones that abolish user fees, are often faced with the realizations of the lack of funding, the unavailability of drugs at health facilities, etc. that further exposes the poor to poverty (Ridde \& Diarra, 2009). Decision-makers expect that new initiatives will improve access to care and provide financial risk protection against the cost of health care services to galvanize use. Evidence relates to the various public health programs, either in place or in a process of implementation, like the implementation of national health insurance schemes and the removal of user fees for reproductive health care services. Such programs are for example the exemptions of user fees for ANC, partial exemptions for Cesarean sections (C-sections) and deliveries in Burkina Faso; exemptions for ANC, childbirth, postnatal care; hospital fee waivers for the poor in Ethiopia; user fee exemptions for facility-based childbirth services in Ghana, Senegal and Sierra Leone (De Allegri et al., 2011; McKinnon, Harper, \& Kaufman, 2015; Witter, Arhinful, Kusi, \& Zakariah-Akoto, 2007). There are also sporadic user fees exemptions in Nigeria, such as exemptions for ANC, childbirth, emergency obstetric care in River State, Kano State (Edu, Agan, Monjok, \& Makowiecka, 2017; Galadanci, Idris, Sadauki, \& Yakasai, 2010; Odeyemi \& Nixon, 2013) and exemptions for C-sections in Mali (El-Khoury, Hatt, \& Gandaho, 2012). Niger, through an NGO fund, implemented a waiver for pregnant women and under-five children while Senegal implemented exemptions for childbirth care and C-sections (Witter, Dieng, Mbengue, Moreira, \& De Brouwere, 2010). The implementation of waivers and exemptions from fees are known to be limited by administrative incapacity, the attitude of the health workers and a lack of knowledge of the guidelines, discrepancies in granting of exemptions and identification of eligible poor (Mohammed \& Dong, 2012; Olakunde, 2012; Russell \& Gilson, 1997).

Sustainable reductions in inequalities in the use of reproductive health care services, not only in Ghana and Nigeria but across the Sub-Saharan African region, can start by improving access to the health care system for all social groups. However, the availability of reproductive health care services does not guarantee that the services will be utilized as intended by the population in need. The available research on reproductive health care services in Sub-Saharan Africa has largely provided information on the probability of use of services (Amoako Johnson, Padmadas, \& Matthews, 2013; Fagbamigbe \& Idemudia, 2017; Idowu, Olowookere, Abiola, Akinwumi, \& Adegbenro, 2017), besides coverage between wealth groups (Hosseinpoor et al., 2011; Hounton et 
al., 2015). Plenty of literature is available on maternal mortality (Anya, Hydara, \& Jaiteh, 2008; Decker \& Constantine, 2011; Okonofua, Ntoimo, \& Ogu, 2018; Onah, Ikeako, \& Iloabachie, 2006; Shimamoto \& Gipson, 2017) and even more on user fees as a barrier to reproductive health care services (Asante, Chikwama, Daniels, \& Armar-Klemesu, 2007; Odeyemi \& Nixon, 2013; Onarheim, Taddesse, Norheim, Abdullah, \& Miljeteig, 2015; Perkins et al., 2009). Only limited evidence is available on the scale of inequality in the use of reproductive health care services for fecund women. However, policies are neither based on evidence about the magnitude or trend of inequality nor based on the variations among women with equal needs. There is missing information on how the actual socioeconomic and demographic characteristics sustain the inequalities in the use of reproductive health care services. Such information is needed to provide evidence on the unequal use of reproductive health care services.

\subsection{STUDY CONTEXT: HEALTH CARE IN GHANA AND NIGERIA}

Ghana and Nigeria are similar as well as different in terms of general country characteristics, the structure of the health care system, and reproductive health care policies. The key country characteristics are outlined below.

\subsubsection{General characteristics of Ghana and Nigeria}

The Republic of Ghana is a Western African country situated on the Gulf of Guinea. Ghana has a land area of $238,537 \mathrm{~km}^{2}$ and is bordered by three French-speaking countries: Togo on the east, Ivory Coast (Côte d'Ivoire) in the west and Burkina Faso in the north. Until recently, there were ten administrative regions: Western, Central, Volta, Eastern, Ashanti, Brong Ahafo, Northern, Upper East, and Upper West and Greater Accra region, with Accra as the capital. Six new regions have recently been created; Bono East, North East, Ahafo region, Western North, Savannah, and Oti regions. Table 1.1 summarizes selected macro indicators based on the World Bank data. Specifically, the GDP growth was $2.8 \%$ in 2014 . The 2019 population estimate stood at 30,417,856, of which $49.3 \%$ were female. The urban population was $51 \%$ in 2010 with annual growth rate of $3.8 \%$ (World Bank, n.d.). The largest contributor to the Ghanaian economy is the service and industry sectors, previously the agriculture sector. Although $41 \%$ of the workforce provides services, $45 \%$ of the working individuals are agrarians and are engaged in the informal sector (GSS, GHS, \& ICF, 2015). About 31.9\% of the population in 2005 was estimated to live below the national poverty line and this share declined to $24.2 \%$ in 2012. Ghana's total expenditure on health as a percentage of Gross Domestic 
Product (GDP) was $4.62 \%$ in 2009 and $3.26 \%$ in 2017.

The Ghana Health System has a Ministry of Health which is in charge of the general coordination, oversight of the health care system, and other functions like policymaking, regulation, planning, coordination, and other central-level activities related to public health (Couttolenc, 2012). Directly below is the Ghana Health Service (GHS), formed under the Ghana Health Service and Teaching Hospitals Act 525. The GHS is almost a self-governing agency with the mandate to "ensure access to health services at the community, sub-district, district, and regional levels." (GHS, 2017). The GHS also has the responsibility for managing and operating nearly all public facilities and is authorized to provide comprehensive and accessible health service throughout the regions. It has a district-level structure and facilities. The GHS has regional health administration offices that provide secondary hospital care through regional hospitals, and coordinate the districts' health activities and planning (Couttolenc, 2012). There are also local governments that are charged with the provision of support for the Ministry of Health and the GHS for some public health services through districts (health centers and other primary care facilities) and sub-districts (community-based health planning and services). Another feature is the non-government sector such as the Christian Health Association of Ghana, which has its own health facilities and health training institutions run through the Church. The traditional methods, including traditional medicine providers, alternative medicine, and faith healers, are also represented in the health arena of Ghana. Other health institutions, which do not cater to the general public, are available for the military and other armed forces, as well as selected universities. The private sector is an important sub-system that is widely available in the country especially for the provision of maternity care.

Nigeria is also a Western Africa country occupying a land area of 923,768 square kilometers. The country is bounded on the North by Niger and Chad, in the East by Cameroon, and the Republic of Benin on the west. Nigeria is the most populous African country with a total population estimate of 200,963,599 in 2019 (World Bank, n.d.). World Bank data summarized in Table 1 show that about $49 \%$ of the total population is female. The annual urban population growth rate as of 2013 was about $4.5 \%$ in Nigeria. The annual GDP growth was 0.58\% in 1999 and 6.67\% in 2013 (World Bank, n.d.). The country has 37 states including the Federal Capital Territory, Abuja. These are grouped into six geopolitical zones: North Central, North East, North West, South East, South South, and South West. There are 774 constitutionally recognized local government areas. The main source of revenue of Nigeria's economy is from its oil and gas, contributing $14 \%$ to the GDP, while the industry sector contributes $18 \%$, the 
services sector contributes $20 \%$ and agriculture contributes $39 \%$. Other contributing sectors are finance and insurance 3.4\%, communications $7.1 \%$, transportation $2.7 \%$, and utility components 2.9\% (NPC \& ICF, 2014). Going by the World Bank data, the national poverty rate, i.e. the proportion living on less than the value of the national poverty line, was $48.4 \%$ in 2003 and declined slightly to $46 \%$ by 2009 . As of 2017 , Nigeria's total expenditure on health as a percentage of GDP was 3.7\%, a slight change from $3.58 \%$ in 2009 . As of $2012,31 \%$ of the total health expenditure came from general government expenditure, 69\% from private sources (WHO, 2014). Household out-ofpocket expenditure remains the largest source of health expenditure in Nigeria.

Table 1.1: Comparison of case study countries characteristics

\begin{tabular}{|c|c|c|}
\hline Characteristic & Ghana & Nigeria \\
\hline Land area $\left(\mathrm{km}^{2}\right)$ & 238,537 & 923,768 \\
\hline \multicolumn{3}{|c|}{ GDP growth (annual \%): } \\
\hline 1999 & 4.40 & 0.58 \\
\hline 2003 & 5.30 & 7.37 \\
\hline 2008 & 9.15 & 6.70 \\
\hline 2013 & 7.30 & 6.67 \\
\hline 2016 & 3.40 & -1.67 \\
\hline \multicolumn{3}{|l|}{ Total population: } \\
\hline 1999 & $18,812,359$ & $119,260,063$ \\
\hline 2019 & $30,417,856$ & $200,963,599$ \\
\hline \multicolumn{3}{|c|}{ \% Female population: } \\
\hline 1999 & 49.50 & 49.60 \\
\hline 2019 & 49.30 & 49.30 \\
\hline \multicolumn{3}{|c|}{ Rural growth rate: } \\
\hline \multicolumn{3}{|c|}{ Urban growth rate: } \\
\hline \multicolumn{3}{|c|}{ Poverty headcount ratio (\% of population) } \\
\hline 2003 & & 48.40 \\
\hline 2005 & 31.90 & \\
\hline 2009 & & 46.00 \\
\hline 2012 & 24.20 & \\
\hline \multicolumn{3}{|c|}{ Total health expenditure (\% of GDP) } \\
\hline 2009 & 4.62 & 3.58 \\
\hline 2017 & 3.26 & 3.75 \\
\hline
\end{tabular}

Note: Data extracted from World Bank website https://data.worldbank.org/?locations=NG-GH (World Bank, n.d.)

The Nigerian health care system has tertiary, secondary and primary levels. The Federal Ministry of Health is in charge of policy development, regulation, and the framework for the delivery of services by federal health institutions, parastatals, and health programs 
at the national level. The State governments provide secondary health care to the population and the local governments are saddled with delivering primary health care. The private sector is controlled by the government, although most private health facilities are suspected to function without the appropriate authorization from the States' Ministries of Health. The informal health sector includes traditional medicine/ healers, midwives, and individual medicine sellers, existing alongside modern medicine in the provision of health care to the population.

\subsubsection{Overview of policies for reproductive health care services in Ghana and Nigeria}

Progress in the use of reproductive health care services across all social strata could greatly avert diseases and death especially among disadvantaged women in SubSaharan Africa. Ghana and Nigeria continue to develop interventions to improve maternal health. There have been a series of policies and programs to address this issue (Koduah, Agyepong, \& van Dijk, 2016; Onwujekwe, Obi, \& Uzochukwu, 2016).

In Ghana, free ANC in government facilities was first introduced in October 1963 (Koduah, van Dijk, \& Agyepong, 2015). More recently, there is a national user fee exemption for maternal care. This was initially implemented in the four poorest regions of Ghana - Northern, Upper East, Upper West, and Central. It was scaled up in 2005 to include all women, covering free childbirth and C-section births. The scale-up took place together with the establishment of the National Health Insurance Scheme (NHIS), which became operational in 2005. The NHIS is mainly financed through national tax revenues, social security taxes for formal sector workers and individual insurance premiums. The scheme experienced funding difficulties, as well as loopholes in ownership of insurance and the limited application of exemption clauses particularly affects women. This led to the establishment of the Maternal Health Care Program in 2008. This program exempts all pregnant women from user fees after registration with the NHIS. Specific for women, the program covers six ANC visits, childbirth care, and two postnatal care visits within six weeks after childbirth (Singh et al., 2015).

In Nigeria likewise, the National Reproductive Health Policy of 2001, approved in 2010, emphasizes the availability and access to good quality reproductive health including family planning information and maternal care services (FMoH, 2009). The policy also targets halving maternal mortality to about 400 deaths per 100,000 live births and doubling access to blood transfusion as well as reproductive health information and services. The NHIS in Nigeria became operational in 2004 through the NHIS Decree No. 35 of 1999. Under the decree, the health facilities registered under the insurance scheme are able to render the capitation on payment or charge the users for services rendered. Alluding to reproductive health care in Nigeria, the decree provides family 
planning, ANC, postnatal care and maternity care for up to four live births. There has been a lag in the expansion of the NHIS to achieve considerable coverage since it became operational. Only the formal sector benefits from a mandatory membership ( $\mathrm{FMoH}, 2012)$. Since the NHIS became operational, it is reported that about $97 \%$ of fecund women were not covered by the scheme (Okebukola \& Brieger, 2016; Okpani \& Abimbola, 2015). To achieve improved reproductive health, in 2008, the federal government implemented a National Health Insurance-Millennium development Goal Maternal and Child Health Project in selected states (NHIS, 2012). This project contained an exemption program for pregnant women, covering basic antenatal services but ended in 2015

In 2000, Ghana fully launched the Community-Based Health Planning and Services Program which has improved access to health care services as its core. This program was based on the results of the 1994 Navrongo Community Health and Family Planning Project piloted in the northern part of the country to illuminate how cultural resources can be used in interventions to promote family planning in the context of African culture (Nyonator, Awoonor-Williams, Phillips, Jones, \& Miller, 2005; Phillips, Bawah, \& Binka, 2005). The Community-Based Health Planning and Services Program recruits, trains and deploys community health officers to fill the differences in access to health care services between rural and urban locations. The community health workers support the health sector in providing supervised childbirth, antenatal and postnatal care. The program also focuses on the provision of family planning using a door-todoor strategy to publicize information targeting both genders. The labels community health volunteers, traditional birth attendants, village health volunteers, community nurses, community-based health workers, amongst others, are broadly used to refer to community health workers in Ghana. They can be community representatives with little association with the formal health care system or trained supporters who are recognized by the authorities or non-governmental organizations (Phillips et al., 2005). The community health workers contribute to augmenting reproductive health care services in Ghana (Phillips et al., 2005). However, in addition to the low motivation among community health workers, the scope and service provided are unclear due to the shortage of health workers in Ghana (Baatiema, Sumah, Tang, \& Ganle, 2016)

Likewise in Nigeria, the Midwives Service Scheme, established in 2009, is an attempt to increase the coverage of skilled birth attendants, especially in rural areas where the newly trained midwives are deployed (Abimbola, Okoli, Olubajo, Abdullahi, \& Pate, 2012). The scheme was set up in Nigeria to boost the available human resources for health with a focus on primary care in rural communities. This is based on the 
evidence that midwives contribute to improvements in the utilization of reproductive health care services (Campbell, Graham, \& group., 2006). The midwives, who are either retired, unemployed or recent graduates, are briefly trained prior to being posted to various primary health centers in rural communities. Usually, these primary health centers are in remote areas with a population of over 10,000 people. Similar to Ghana's Community-Based Health Planning and Services Program, the Midwives Service Scheme in Nigeria focuses on rural and hard-to-reach geographical areas. However, the scheme was implemented without a pilot and faces a number of challenges including the availability of qualified midwives and retaining their services (Abimbola et al., 2012).

Though there has been an improvement in the reproductive health care sector in both Ghana and Nigeria (Figures 1.1-1.3), it appears that the government in Nigeria compared with that in Ghana funds reproductive health interventions and programs spasmodically. At present, there is no national user fee exemption for reproductive health care services in Nigeria. Family planning and maternal care interventions are provided at the discretion of the State governments. The State administration of the day may well decide to provide subsidized or entirely free reproductive health care services through the community primary health care, although such initiatives usually cover selected services and a limited time period. Some states include registration and consultation costs, antenatal, childbirth and postnatal care in the fee exemption list and others select services along the continuum of care for reproductive health care services. Therefore, women in Nigeria pay differently for reproductive health care depending on the State (Edu et al., 2017; Fabamwo \& Okonofua, 2010).

Moreover, policy decisions in Nigeria are not always based on evidence. For instance, the Subsidy Reinvestment and Empowerment Programme (SURE-P) implemented in 2012, was funded from the revenues of fuel subsidy reduction. The program included maternal and child health (SURE-P/MCH) and engaged community health workers such as community health extension workers and volunteer village health workers working in remote areas to improve access to quality health services (Mirzoev et al., 2016). The program has been evaluated as being unstructured in defining and identifying the poor and vulnerable groups (Amakom, 2013).

Several isolated interventions exist to address the issue of family planning and maternal health care services, mostly addressing the supply-side, especially in Nigeria more than in Ghana. Such inconsistencies would have equity implications and create uneven access for women as a result of differences in the ability to obtain reproductive 
health care. Initiatives to effectively deliver maternal health improvement strategies are questionable due to inappropriate or total absence of strategies that ensure safe motherhood across board (Ijadunola, Ijadunola, Esimai, \& Abiona, 2010).

\subsection{AIM AND OBJECTIVES OF THE DISSERTATION}

The existence of a steep wealth-gradient in the use of health care services across SubSaharan Africa obscures the significance of other user-related health determinants that are responsible for the disparities in health outcomes observed. The lack of comparative studies for resource-constrained settings makes this topic particularly important for research. Cross-country evidence is needed to understand the ways a group position determines service use. This is key to identifying barriers to equitable care, which vary with health care system characteristics, social context, social groups, and type of services. Furthermore, an understanding of the determinants of service use gives decision-makers scientific evidence for policy formulation. In particular for reproductive health care, identifying society-wide inter-group inequalities is crucial for designing health and social policies that have the potential to improve maternal health outcomes. As outlined in the previous sections, such evidence is largely lacking in Sub-Saharan Africa.

Therefore, the central aim of this dissertation is to increase our knowledge and understanding of the determinants of inequalities in reproductive health care in Sub-Saharan Africa, taking Ghana and Nigeria as case studies. More precisely, the dissertation aims to explore the association between inequalities in the utilization of reproductive health care and society-wide inter-group inequalities among women in Ghana and Nigeria. The comparison between the two countries and to other countries in the region is also a part of the dissertation aim.

Given the dissertation aim, the following research questions are addressed:

1. What evidence is there on the inequalities in the utilization of reproductive health care services in Sub-Saharan Africa and what is their association with socioeconomic factors?

This research question is related to socioeconomic inequality in reproductive health care use and the identification of contextual differences across SubSaharan African countries. It is important to reflect on changes in policies and service coverage, which may focus on one or more reproductive health care services, increasing the timely use at the expense of other care services (Hercot 
et al., 2011; McKinnon et al., 2015; Richard et al., 2013; Witter et al., 2007). Also, there is a need to outline the recent evidence that illuminates the gravity of wealth inequality in the use of reproductive health care services. Previous studies have shown positive, negative, or no associations between wealth inequality and the use of reproductive health care service. The goal is to systematically review and analyze the evidence and to identify inequalities in various aspects of reproductive health care services. Thus, a review can outline the overall scale of inequalities in reproductive health care service use. In addition, cross-national differences in the extent of the inequality across different reproductive health care service categories in Sub-Saharan African countries can explain the magnitude and variation in disparity in the use of reproductive health care. Meta-analyses were performed to calculate the effect sizes of outcome measures. Meta-regression was performed to model the source of heterogeneity and to identify cross-national differences in magnitudes across service categories. The evidence produced informs of how the magnitude of inequality in reproductive health care use varies with the type of service across Sub-Saharan Africa.

2. What are the patterns of reproductive health care service use in Ghana and Nigeria and what are the differences and similarities between the countries?

The use of preventive and medical reproductive health care differs between women. For women, these services are obtainable from traditional or untrained providers, or they can be administered by clinicians and professionals at hospitals and other medical health services centers (Darroch \& Singh, 2013). Therefore, there are differences in efficacy. Multiple studies have analyzed the probabilities of the use of family planning services and maternal health care services within a population (Adamu \& Salihu, 2002; Johnson, Frempong-Ainguah, \& Padmadas, 2016; Johnson, Padmadas, \& Brown, 2009; Onah et al., 2006). Our goal is to provide a better understanding of the types of reproductive health care services accessed by women with different sociodemographic and economic characteristics. This would depict the disparities in the adequacy of the services provided to and received by population sub-groups. Therefore, the use of family planning and maternal care by women in Ghana and Nigeria is to be examined in this study. For this, population-level data and two-step cluster analyses identify key user groups and the patterns of reproductive health care use among women of reproductive ages in both countries. Multinomial logistic regression is subsequently used for the analysis of factors associated with the cluster membership identified. The evidence generated clarifies the connection between reproductive health care service utilization by type and socioeconomic differences in two seemingly similar 
environments.

3. How did the inequalities in reproductive health care service use in Ghana and Nigeria change over time?

It is suggested that evidence of an association of wealth inequalities exists in a single year's cross-section and also that the income inequality hypothesis is at best over-rated (Hatt, Makinen, Madhavan, \& Conlon, 2013). Previous research suggests that new health policies are beneficial to the wealthy population only, consequently widening inequalities (Victora et al., 2018). Health interventions focusing on reproductive health care services in Ghana and Nigeria have been ramped up in recent times. Therefore, our aim is to study changes over time in wealth inequalities in the use of reproductive health care services to assess whether any progress is being made in reducing inequities. This study addresses the inverse equity hypothesis. In particular, the investigation explores if health interventions to reduce inequality in access to reproductive health care services, benefit the entire population across the wealth-gradient, or only sub-groups. Using concentration curves and concentration indices, our goal is to provide insight into horizontal inequalities by investigating the changes in inequalities in using reproductive health care services over time in Ghana and Nigeria. Cross-sectional data going back almost 20 years are examined. Analysis of inequality trends over time helps to indicate variation in the prevalence of horizontal inequality in access to health service and to draw a conclusion on the intervention impact on different subpopulation groups within the countries.

4. How do the inequalities in reproductive health care service use in Ghana and Nigeria compare to those in other countries in the West Africa region?

In this dissertation, attention is paid to inequalities that enable other inequalities. User fees are one of the factors that create a disparity in access to reproductive health care services in Sub-Saharan Africa. The objective is to investigate how much each determinant of inequality contributes to the inequality caused by wealth and compare the contribution of each inequality within- and across countries. The hypothesis is that in addition to variation in use due to wealth, other determinants of use are germane. The gap in reproductive health care use in Burkina Faso, Ghana, Niger, Nigeria, and Senegal is the focus of this study. Not only do these West African countries have a relatively high maternal mortality rate, but a variety of interventions to address reproductive health care utilization rates have been designed and implemented in these countries (Meessen et al., 2009; Richard et al., 2013; Ridde \& Diarra, 2009). Using nationwide survey data for Burkina Faso, Niger, 
Nigeria, Ghana, and Senegal, decomposition analysis is performed to quantify the contribution of sociodemographic characteristics to observed disparities in reproductive health care service use. In particular, decomposition analysis quantifies the contribution of sociodemographic characteristics to disparities in the utilization of reproductive health care services. When analyzing the effects of other determinants on-top of wealth-inequality, we also examine if the country has a form of user fee exemption policy.

\subsection{DATASET}

In this dissertation, we use secondary data from the Demographic and Health Surveys (DHS). The DHS Program is one of the principal sources of nationally representative data on population health and the provision of health care services. DHS are crosssectional, comparable and representative population-based surveys that gather a variety of information on reproductive health, nutrition and other demographics of respondents from low-middle income countries (DHS, 2018). The surveys are conducted under an international program implemented by ICF International and funded by the USAID with contributions from UNICEF, UNFPA, WHO, and UNAIDS (Demographic Health Survey, 2016). The DHS adopts a multi-stage cluster design. Samples selected for enumeration are ensured to be representative and comparative across countries. The DHS involves a two-stage cluster and systematic sampling design with households selected at random. DHS questionnaires and techniques for data collection are publicly available (DHS, 2018).

The dissertation used the DHS surveys available for Ghana (2003, 2008, and 2014) and Nigeria (2003, 2008, and 2013). In the additional cross-country analysis study, we include DHS surveys from Burkina Faso (2010), Niger (2012), and Senegal (2016 DHS-VII). All five DHS included in this dissertation are household-based samples. The samples were selected in a similar manner. In particular, the DHS adopted a stratified two-stage cluster design and samples selected for enumeration were ensured to be representative of the countries. The households involved were drawn from a simplified list of households (Agence Nationale de la Statistique et de la Démographie - ANSD/Sénégal \& ICF, 2017; GSS et al., 2015; Institut National de la Statistique et de la Démographie - INSD/Burkina Faso \& ICF International, 2012; Institut National de la Statistique/Niger, UNICEF/Niger, \& ICF International, 2013; NPC \& ICF, 2014).

The sampling accounted for differences in population distribution regionally as well 
as for the urban-rural spread. Designated households were enumerated without allowance for a change or replacement to prevent bias. Respondents were selected on the basis of being female (for the female survey) or male (for the male survey), aged 15-49, and whether the respondent was a usual member of the household or having spent the night prior to the survey in the household. All eligible women aged 15-49 were interviewed with the Women's Questionnaire. The Women's Questionnaire was used to collect respondent's individual characteristics including age, marital status, occupation, residence as well as other information on topics including; reproductive history; contraceptive knowledge and use; antenatal, childbirth and postnatal care; marriage; attitudes about family planning. We used data for women who have had at least one birth in the 5 years prior to the survey.

Wealth index is readily available in all DHS datasets. It is constructed based on household assets and utility services, i.e. the possession of some goods and affordability of some services that have been associated with wealth including country-specific items. A wealth scale is then computed using ownership or non-ownership of such goods and selected assets (Rutstein \& Johnson, 2004). The DHS constructs a wealth index score and computes the household wealth (in quintiles) as a socioeconomic indicator based on the possession of assets or other variables including type of flooring, wall and roofing materials, electricity, toilet, water source, possession of radio, television, refrigerator, bicycle, motorcycle, car or truck, and mobile phone. This has been described as the local perception of wealth approach.

\subsection{OUTLINE OF THE DISSERTATION}

After this introductory chapter, Chapter 2 presents a review of existing evidence on the extent to which the unequal distribution of income and wealth fosters inequities in accessing and using reproductive health care services. Specifically, based on the method of meta-analysis, the chapter illuminates the gravity of wealth inequality in the use of reproductive health care services in Sub-Saharan African countries. The next two chapters focus on Ghana and Nigeria specifically. Chapter 3 examines the differences in the use of reproductive health care services by women in Ghana and Nigeria and their variation across sociodemographic and economic groups. The patterns of use of family planning and maternal care by women in Ghana and Nigeria are examined. For this purpose, the method of cluster-analysis is used to identify access-related patterns. In particular, we use population-level data and twostep cluster analyses and multinomial logistic regression. Chapter $\mathbf{4}$ investigates 
horizontal inequalities in the use of reproductive health care services in Ghana and Nigeria by examining the changes in the determinants of inequalities over the years. Concentration indices are estimated per service, country and year to examine trends. This chapter uses concentration curves and concentration indices to measure the magnitude of socioeconomic inequalities and horizontal inequalities in the use of reproductive health care services. Chapter 5 again takes a cross-country perspective in order to compare the situation in Ghana and Nigeria to that in other countries in the West African region. Specifically, by applying the method of decomposition analysis, the chapter investigates relevant contributors to disparities in reproductive health care utilization in five West African countries. We performed decomposition analyses on countrywide survey data for Burkina Faso, Niger, Nigeria, Ghana, and Senegal to quantify the contribution of sociodemographic characteristics to the disparities in reproductive health care services use. Chapter 6 outlines and discussed the main findings of the dissertation, as well as study implications for policy and research. 



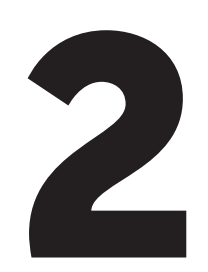

\section{INEQUALITIES IN REPRODUCTIVE HEALTH CARE SERVICES ACROSS SUB-SAHARAN AFRICA. A SYSTEMATIC REVIEW AND META-ANALYSIS}

\section{Draws upon:}

Ogundele, O. J., Pavlova, M., \& Groot, W. (2020). Socioeconomic inequalities in reproductive health care services across Sub-Saharan Africa. A systematic review and meta-analysis. Sexual \& Reproductive Healthcare, 25, 100536 


\section{ABSTRACT}

\section{Background}

Women in Sub-Saharan African experience socioeconomic barriers in the use of reproductive health care services. This chapter analyzes the evidence on socioeconomic inequalities in reproductive health care utilization in Sub-Saharan Africa and identifies the variance in the estimates of these inequalities.

\section{Methods}

We performed a systematic review and meta-analysis of studies on socioeconomic inequalities in the use of reproductive health care services published in English between January 2008 and June 2019. We used meta-regression to identify sources of heterogeneity in reproductive care services use.

\section{Results}

Twenty-two studies were included in the meta-analysis and they reported 305 estimates of the concentration index for different countries and different reproductive health care services. We grouped the services into ten categories of reproductive health care services. Results show that socioeconomic status was associated with inequality in reproductive health care use and was on average high, with a pro-wealthy inequality magnitude of the concentration index of 0.202 . The meta-analysis indicated that pro-wealthy inequality was highest for skilled childbirth services with an average concentration index of 0.343 . Family planning and contraceptive services had an average concentration index of 0.268 and 0.203 respectively. The overall concentration index for antenatal services and the components of antenatal care was 0.164 and 0.142 respectively. Random-effects meta-regression showed that the heterogeneity in reproductive health care use was explained by contextual differences between countries. Cross-national differences in the magnitude of inequality in reproductive health care services were largest in Cameroon and lowest in Namibia.

\section{Conclusions}

Our findings show that the magnitude of inequality in reproductive health care use varies with the type of service and that the focus on skilled childbirth services through user fees removal appears to have fostered inequality. The one-size-fits-all approach to reproductive health care initiatives has ignored differences in reproductive health care needs and the ability to overcome use barriers by women in various socioeconomic groups. Context-specific interventions that are needs-based and address socioeconomic inequalities are needed. 


\subsection{BACKGROUND}

The Millennium Development Goals (MDGs) did not substantially address the within and between-country inequalities in access to reproductive health care services, which the SDGs now aim to tackle (WHO, 2015a). The MDGs, however, highlighted socioeconomic disparities in the utilization of maternal health care. They also brought the focus on unfair distribution of reproductive health care services, particularly on the low utilization of skilled childbirth services in Sub-Saharan Africa where the lowest improvement in coverage was recorded and the highest rates of maternal mortality occur (WHO, 2005a). Consequently, the agenda remains unfinished; inequalities within populations and between populations persist. The SDG agenda now includes, as a priority, targets to curb rising inequalities. Target 5.6 calls for ensuring universal access to sexual and reproductive health and reproductive rights (UN, 2015).

International and national efforts are being directed to improve women's access to reproductive health care services in Sub-Saharan Africa. International stakeholders complement the efforts of national governments through monetary and material assistance for policy execution (Hsu, Berman, \& Mills, 2013), as well as by establishing population-based surveillance for understanding the trends and distribution of reproductive ill-health (AbouZahr \& Vaughan, 2000). What is missing is research on socioeconomic inequalities that explains variations in the availability and quality of reproductive health care (Kendall \& Langer, 2015), as also explained in Chapter 1. Given the SDGs, to address rising inequalities in health, evidence on disparities in reproductive health care is essential. Such evidence could foster a more efficient allocation of scarce resources and a more equitable distribution of reproductive health care services. Despite the absence of conclusive evidence on equity in the distribution of health care, some Sub-Saharan African countries have begun to either reduce or waive user fees for all or specific reproductive health care services. The objective is to diminish the burden of out-of-pocket costs across socioeconomic gradients and to increase access to health care services (Aikins, Aryeetey, Dako-Gyeke, Adongo, \& McGough, 2015; Hatt et al., 2013; Richard et al., 2013). In addition, the objective is to scale-up other programs, like community health workers schemes, midwife schemes, and skilled attendance at birth (Baatiema et al., 2016; Filippi et al., 2006; Onwujekwe et al., 2016). However, equity in the use of the health care services provided, varies especially when the services relate to the poor (McKinnon et al., 2015; Ravit et al., 2018). The overall equity effects of efforts to improve family planning and maternal health services, mainly facility-based childbirth, are unclear. Particularly, their effectiveness in removing the financial burden as a barrier to maternal care access is largely unknown. 
There is also no consensus on the scale of the inequalities in service use. Studies have reported various magnitudes of inequality in the utilization of reproductive health care services taking account of recent changes in policies (De Allegri et al., 2011; McKinnon et al., 2015; Ravit et al., 2018; Ridde et al., 2015). Further, studies on the association of socioeconomic status and the use of various reproductive health care services have mostly found persisting pro-rich inequity (Johnson et al., 2016; Makoka, 2009; Mezmur, Navaneetham, Letamo, \& Bariagaber, 2017; Nghargbu \& Olaniyan, 2017). Other studies have argued that pro-poor reproductive health care initiatives do not benefit women with greater needs due to inefficient targeting (Victora et al., 2018; Zere, Moeti, Kirigia, Mwase, \& Kataika, 2007) and arbitrary prioritization of services (Witter, 2009). For efficient and sustainable inequality-reducing strategies, policymakers need the best evidence currently available.

Despite the evidence on the various barriers to using reproductive health care services (Simkhada, Teijlingen, Porter, \& Simkhada, 2008) and the burden of reproductive illhealth (Ezeh et al., 2016), there remains a significant gap in our knowledge about the equity effects of prioritizing reproductive health care services across Sub-Saharan African countries. It has been theorized, that user fee waiver policies will benefit all women, especially socioeconomically disadvantaged women, and will promote the use of reproductive health care services (Ridde, Kouanda, Bado, Bado, \& Haddad, 2012; Ridde $\&$ Morestin, 2011). It is yet not clear whether this holds true across different countries and different reproductive health care services (McKinnon et al., 2015; Nyonator \& Kutzin, 1999). It is also not clear whether there are socioeconomic disparities in the use of reproductive health care services that remain outside the fee waiver policies. What is known is that policy choices may result in unfair differences in access to health care services and health outcomes among women (Bonfrer, van de Poel, Grimm, \& Van Doorslaer, 2014; Fleurbaey \& Schokkaert, 2009), in particular, among women who need reproductive health care services, which are exempted from user fees, and women who need other reproductive health care services, which are not prioritized for exemption. Therefore, it is crucial to determine the equity effects of policies, such as exemption policies, across the continuum of reproductive health care services within a country in the context of the prevailing health system. Given this knowledge gap, this chapter focuses on providing insights into which reproductive health care services in SubSaharan African countries are associated with socioeconomic inequalities.

The study contributes to our knowledge by outlining the association between socioeconomic inequality and reproductive health care services utilization based on the empirical evidence available for Sub-Saharan African countries. The services 
provision could be improved based on evidence on where reproductive health care is most needed and which population group is most vulnerable. However, priority setting solely based on information on the burden of disease or chances of reproductive health care services use could be misleading for the prioritization and provision of services. Evidence on socioeconomic inequality is also needed for adequate prioritization in the area of reproductive health care.

Therefore, it is vital to elucidate the socioeconomic inequality in reproductive health care services among women in Sub-Saharan African countries. Most policies have focused on specific maternal health services rather than on the whole of the continuum of care for maternal, newborn and child health (Hatt et al., 2013). To effectively and efficiently improve reproductive health outcomes, equity in the provision of prepregnancy, pregnancy, emergency obstetrics, and postpartum services is required, not just equity in childbirth services, which have been the main focus of reproductive health care policies (De Allegri et al., 2012; Hatt et al., 2013; Witter et al., 2010). The measurement of socioeconomic inequalities is imperative to explain the variation in quality, availability, and use of reproductive health care services. Thus, we contribute to the existing knowledge by comparing the patterns of reproductive health care services inequalities within and across countries with and without national fee waiver policies. These track the progress across countries on reducing equity gaps. Achieving equity in the provision of adequate reproductive health care is important to reduce maternal and child mortality especially among the poor. Therefore, we conducted a systematic review followed by a meta-analysis on inequalities in reproductive health care use.

The aim is to analyze the evidence on socioeconomic inequalities in reproductive health care utilization in Sub-Saharan Africa and to identify the variance in these inequalities. We identify the size and differences in inequality in different aspects of reproductive health care use. This enables us to indicate where interventions should target to improve equity in reproductive health care.

\subsection{METHODS}

This systematic review and meta-analysis were performed according to the Preferred Reporting Items for Systematic Reviews and Meta-Analyses (PRISMA) guidelines (Moher, Liberati, Tetzlaff, \& Altman, 2009). 


\subsubsection{Search strategy and selection criteria}

We searched PubMed, POPLINE and JSTOR databases for studies that tested the association of socioeconomic position with access/use of reproductive health care services among women living in any Sub-Saharan African country. A population, exposure, comparator, outcome (PECO) statement was used to define the research question and to develop the search terms, as well as inclusion and exclusion criteria for the systematic review. The PECO statement was defined as follows: women of reproductive ages in Sub-Saharan Africa $(P)$, reproductive health care services $(E)$, the (level of) use/access/exposure (C), and inequality levels measured by concentration indices (O). The search terms consisted of four blocks: "inequalities", "access or use", "reproductive health", and "Sub-Saharan Africa". Each block combined various synonyms related to that block. The exact search string is detailed in Box 2.1. The search strategy was discussed with a librarian. The three journal databases were searched for titles and abstracts containing these terms. We considered articles published between January 1st, 2008, and June 6th, 2019 (the date of the final search) in all peer-reviewed journals included in the databases. The search was limited to articles in the English language. Research published before January $1^{\text {st }}, 2008$ was excluded because of its insignificance to current inequalities in reproductive health care.

The objective was to select articles reporting quantitative studies that measure access or use of reproductive health care service as well as the concentration index (conindex) as a measure of inequality at the country level. Our inclusion criteria were: 1) the study must have been peer-reviewed; 2) the study must include an analysis of the access and/or use of reproductive health care services using concentration indices; 3) quantitative data should be available or could be derived from the published data or referenced source; 4) the study was published after 2008 in the English language; 5) the study must include women of reproductive ages in a Sub-Saharan African country and should be at the regional or nationally representative level, irrespective of the design of the study. Publications that examine the use of reproductive health care services among women with morbidities, such as HIV, were also excluded. Publications that were not peer-reviewed, like conference proceedings, case reports, dissertations; qualitative studies; journal cover pages, were excluded. 
Box 2.1: Search terms per thematic search block

\begin{tabular}{ll}
\hline \multicolumn{1}{c}{ Search strategy: PubMed } \\
\hline Inequalities & (Healthcare Disparities[MeSH] OR "Health Status Disparities"[Mesh]) OR ((Healthcare[tiab] OR \\
block & Health care[tiab] OR Health service*[tiab] OR Care[tiab]) AND (Barrier[tiab] OR Barriers[tiab] \\
& OR Disparity[tiab] OR Disparities[tiab] OR Equality[tiab] OR Inequalities[tiab] OR Equity[tiab] \\
& OR Equities[tiab] OR Inequality[tiab] OR Inequalities[tiab] OR Inequity[tiab] OR Inequities[tiab] \\
& OR Disadvantage[tiab] OR Disadvantages[tiab] OR Variation[tiab] OR Variations[tiab] OR \\
& Determinant[tiab] OR Determinants[tiab] OR "Socioeconomic Factors"[Mesh]))
\end{tabular}
AND

Access and/ ("Health Services Accessibility"[Mesh] OR Access OR Accessib*[tiab] OR Use[tiab] OR or Use Usage[tiab] OR Utilization[tiab] OR Utilisation[tiab] OR Uptake[tiab] OR Use[tiab] OR Usage[tiab] OR Nonuse[tiab] OR Nonusage[tiab] OR Distribution[tiab])

AND

Reproductive ("Reproductive Health Services"[Mesh] OR Reproductive OR Antenatal OR Prenatal OR Health Maternal OR "Family planning"[tiab] OR "Delivery care" [tiab] OR TBA OR "Traditional birth" [tiab] OR "Family Planning Service"[tiab] OR Contraception[tiab] OR Contraceptive[tiab] OR C-section[tiab] OR "Caesarean section"[tiab] OR "cesarean section"[tiab] OR "Unmet needs" [tiab] OR "unmet need" OR Obstetric[tiab] OR "* birth assistance" [tiab] OR SBA[tiab] OR Skilled delivery[tiab] OR "Maternal health care" [tiab] OR Pregnancy[tiab] OR Abortion[tiab] OR Delivery [tiab] OR "Birth control" [tiab] OR "Postpartum care" [tiab] OR "birth*"[tiab] OR "labour" [tiab] OR "labor" [tiab])

AND

Sub-Saharan ("Africa South of the Sahara"[Mesh] OR "Africa, Central"[Mesh] OR Cameroon [Mesh] OR Africa Central African Republic[Mesh] OR Chad [Mesh] OR Congo [Mesh] OR "Democratic Republic of the Congo"[Mesh] OR "Equatorial Guinea" [Mesh] OR Gabon [Mesh] OR "Sao Tome and Principe"[Mesh] OR "Africa, Eastern"[Mesh] OR Burundi [Mesh] OR Djibouti [Mesh] OR Eritrea [Mesh] OR Ethiopia [Mesh] OR Kenya [Mesh] OR Rwanda [Mesh] OR Somalia [Mesh] OR "South Sudan"[Mesh] OR Sudan [Mesh] OR Tanzania [Mesh] OR Uganda [Mesh] OR "Africa, Southern"[Mesh] OR Angola [Mesh] OR Botswana [Mesh] OR Lesotho [Mesh] OR Malaw [Mesh] OR Mozambique [Mesh] OR Namibia [Mesh] OR "South Africa"[Mesh] OR Swaziland [Mesh] OR Zambia [Mesh] OR Zimbabwe [Mesh] OR "Africa South of the Sahara"[tiab] OR Cameroon [tiab] OR "Central African Republic"[tiab] OR Chad [tiab] OR Congo [tiab] OR "Democratic Republic of the Congo"[tiab] OR "Equatorial Guinea"[tiab] OR Gabon [tiab] OR "Sao Tome and Principe"[tiab] OR Burundi [tiab] OR Djibouti [tiab] OR Eritrea [tiab] OR Ethiopia [tiab] OR Kenya [tiab] OR Rwanda [tiab] OR Somalia [tiab] OR "South Sudan"[tiab] OR Sudan [tiab] OR Tanzania [tiab] OR Uganda[tiab] OR Angola [tiab] OR Botswana [tiab] OR Lesotho [tiab] OR Malawi [tiab] OR Mozambique [tiab] OR Namibia [tiab] OR "South Africa"[tiab] OR Swaziland [tiab] OR Zambia [tiab] OR Zimbabwe[tiab] OR "Africa, Western"[Mesh] OR Nigeria[tiab] OR Niger [tiab] OR Burkina Faso[tiab] OR Senegal[tiab] OR Benin[tiab] OR Cabo Verde[tiab] OR Côte d'Ivoire[tiab] OR Ivory Coast[tiab] OR Gambia[tiab] OR Guinea[tiab] OR Guinea-Bissau[tiab] OR Liberia[tiab] OR Mali[tiab] OR Sierra Leone[tiab] OR Togo[tiab] OR Nigeria[Mesh] OR Niger [Mesh] OR Burkina Faso[Mesh] OR Senegal[Mesh] OR Benin[Mesh] OR Cabo Verde[Mesh] OR Côte d'Ivoire[Mesh] OR Ivory Coast[Mesh] OR Gambia[Mesh] OR Guinea[Mesh] OR Guinea-Bissau[Mesh] OR Liberia[Mesh] OR Mali[Mesh] OR Sierra Leone[Mesh] OR Togo[Mesh])

\section{Search strategy: POPLINE}

Search term: maternal care

Filter by: (1) Research report, (2) Socioeconomic status and, (3) Region: (3.1) Africa, and (3.2) Africa sub-Saharan

\section{Search strategy: JSTOR}

Search term: (( ((ti: (Maternal OR obstetric OR family planning OR contraceptive) AND (care OR services)) AND (utilization OR access OR use)) AND (socioeconomic OR economic)) AND (africa)) Filter by: From 2008 
During the initial search, titles and abstracts identified in the search were independently screened by the lead researcher and one of the supervisors to exclude publications that were irrelevant based on the above inclusion and exclusion criteria. The two researchers did this screening and the results were compared. Full-text versions were obtained for all titles that remained after the initial screening. Obtained full-text articles were read and those not satisfying the inclusion criteria were subsequently removed. The remaining articles were included in the systematic review. The reference lists of all included articles were hand囚searched for additional relevant publications. The selection process was regularly discussed with all contributing researchers.

Relevant information was extracted from the articles and was stored on a customized data extraction sheet by the lead researcher with contribution from the two supervisors. All steps of the data extraction and the related output were discussed with the other two contributors to clarify questions and unclarities. Extracted data included study country, year of publication, study design, sample size, sample year, population attributes, outcome magnitude, outcome direction, point estimates and precision measures.

The outcome measure was the estimate of the concentration index as a measure of inequality in the access or use of reproductive health care services. The concentration index measures inequality in one outcome variable over the distribution of another usually wealth-related variable in the range of -1 to +1 . The concentration index is zero in the absence of socioeconomic-related inequality, negative when the outcome is more observed among the poor and positive when the outcome is more observed among the rich (Kakwani, Wagstaff, \& vanDoorslaer, 1997). Some articles included in the review reported on more than one outcome measure and therefore several outcomes per article were recorded. Studies that analyzed the same data source but use different samples and/or reproductive health care services were included. Studies that overlapped in data source, year, sample, and reproductive health care services were identified. Thus, the studies that had the most complete data or a larger sample size were kept. We excluded results from studies that provided magnitudes of socioeconomic inequality in quartiles or another sub-group categorization.

We included studies that examined absolute and relative concentration indices. Both indices take into account the level of utilization of reproductive health care services within the population measured. As described in Chapter 1, the absolute concentration index captures both information on the level and the distribution of a variable, unlike the relative concentration indices which only takes into consideration how much 
the level of utilization varies within the population (Clarke, Gerdtham, Johannesson, Bingefors, \& Smith, 2002)

\subsubsection{Statistical analyses}

The scientific quality of each included study was assessed by the lead researcher and one of the supervisors using the Effective Public Health Practice Project's (EPHPP) quality assessment tool for quantitative studies (Effective Public Health Practice Project, 1998). The EPHPP tool rates eight study components, including selection bias, study design, confounders, blinding, data collection methods, withdrawals and dropouts, intervention integrity and analysis. Each component is rated as either good, fair or poor (Effective Public Health Practice Project, 1998). Thus, studies included in this review were given an overall methodological rating of high, moderate, or low, based on the aggregate of the ratings of the individual components.

Articles reported on a variety of reproductive health care services. Drawing from the works of Kerber et al. (2007), we distinguished 10 reproductive health care services. These were family planning services; HIV services; contraceptive services; ANC services; four or more ANC visits; adequate components of ANC; inadequate components of ANC; skilled childbirth services; unskilled childbirth services; and postnatal care services. The reproductive health care services that made up each category can be found in Appendix A1.

To calculate the effect size and carry out the meta-analysis, we followed a method used in a previous correlational meta-analysis (Rosenblad, 2009). In particular, the method requires to transform the correlation coefficients reported in different studies to Fisher's $z$ values in order to generate effect sizes (Gentes \& Ruscio, 2011). Thus, we applied the following steps.

First, we transformed the concentration indices reported in the different studies we reviewed, from their values in the range from -1 to +1 , to Fisher's $z$ values (Clarke et al., 2002; Rosenblad, 2009). We applied the transformation procedure described in previous studies (Gentes \& Ruscio, 2011; Rosenblad, 2009). The transformation to Fisher's z scores and the backward transformations to concentration indices are available as indicated in Equations (2.1 - 2.4) below. Equation (2.1) is the transformation formula from sampling distribution of correlation $r$ to Fisher's $z$. The variance of $z$ is calculated using equation 2.2. And the standard error is derived from equation (2.3). Lastly, equation (2.4) provides the back transformation formula from Fisher's $z$ to sampling distribution of correlation $r$. Our findings with- and without the Fisher's $z$ transformation were similar, therefore 
reported findings are based on the transformed data only.

$$
\begin{aligned}
& z=0.5[\ln (1+r)-\ln (1-r)] \\
& V z=\frac{1}{n-3} \\
& S E z=(V z)^{0.5} \\
& r=E X P^{2 z}-\frac{1}{F . X P^{22}}+1
\end{aligned}
$$

Second, the transformed Fisher's $z$ values were pooled to generate the mean difference of the inequality estimates reported in the included studies. Initially, an overall pooled reproductive health inequality effect size was calculated, then we calculated this for the respective categories of reproductive health care services described above.

Third, based on DerSimonian and Laird's (DerSimonian \& Laird, 1986) random-effects method, meta-analysis was performed using Fisher's $z$ values (transformed concentration index values) as the outcome variable. We undertook the random-effects analysis using a simple linear model. The random-effects meta-analysis is estimated because included studies assess several different but related outcome measures (concentration indices) and this type of meta-regression account for within-study and between-study variations (DerSimonian \& Laird, 1986; Knapp \& Hartung, 2003).

Fourth, we used $I^{2}$ and the $Q$ test of heterogeneity to examine variation among studies included in the meta-analysis. $I^{2}$ describes the proportion of total variation in study effect sizes that is due to heterogeneity and not to sampling error (with $25 \%, 50 \%$, and $75 \%$ indicating low, moderate, and high heterogeneity respectively) (Higgins, Thompson, Deeks, \& Altman, 2003).

Fifth, the results of the meta-analysis were in Fisher's $z$. Therefore, we then converted them back to concentration indices to report the average concentration values and 95\% $\mathrm{Cl}$. For the backward transformation, we again applied the procedure described in previous studies (Gentes \& Ruscio, 2011; Rosenblad, 2009), as also explained in Supplementary A4. The summary effects are thus presented both in Fisher's $z$ scale and in concentration index values.

Finally, we assessed publication bias with funnel plots where standard errors of Fisher's $z$ units were plotted against the effect estimates. We tested the hypothesis of no linear association between the summary effect and its standard error and examined the effects of small studies using Egger's method (Egger, Davey Smith, Schneider, \& Minder, 1997). In addition to the meta-analysis, we also performed random-effect linear meta- 
regression to model the source of heterogeneity with restricted maximum likelihood estimation and the improved variance estimator of Knapp and Hartung (Knapp \& Hartung, 2003). We used the summary effects of the reproductive health care service categories in Fisher's $z$ units as the dependent variable in three random-effects multivariate meta-regression models. Three models were estimated. Each model built on the previous and included an additional set of study-level covariates. Model 1 controlled for categories of reproductive health care services only. Study characteristics (publication year, sample year, sample source, and study quality) were then added to estimate Model 2. In the final model, Model 3, country covariates were added.

The above data analyses were conducted using the metan and metareg packages for meta-analyses and meta-regression respectively, and metabias and metafunnel packages for assessment of publication bias in STATA version 15 (Stata Corp, Lakeway, College Station, Texas, USA) (StataCorp., 2017).

Based on Model 3, we perform post-estimation in EXCEL to identify cross-national differences in magnitudes across service categories. Keeping all other covariates at their reference categories (skilled childbirth services, the publication year 2008 2010, the sample year 2011 - 2016, sample size $>8000$, DHS data or non-DHS, study quality: high, and Ethiopia), we estimated the coefficients of each reproductive health care category per country. Specifically, holding all other variables constant in Model 3, we calculate the expected effect size in each reproductive health care category, i.e. the change in the pooled reproductive health care inequality effect size given a unit change in each reproductive health care category. Subsequently, we calculate the expected effect size in each country variable for each respective reproductive health care category i.e. the change in the pooled reproductive health care inequality effect size given a unit change in each country variable and each reproductive health care category. These estimations are then transformed into concentration index (conindex) values to describe the effects in average magnitudes of inequality. We accommodate for statistically significant predictors of inequality in reproductive health use identified in the meta-regression.

\subsection{RESULTS}

Searches in the databases using the search string produced 4,714 articles (Figure 2.1 shows the flow diagram of study selection for the analysis). Subsequent screening of titles and abstracts resulted in the exclusion of 4,683 , as they were irrelevant or did 
not measure inequality in the outcome of interest. In total, 31 articles were of interest and their full-texts were retrieved and assessed for eligibility using the inclusion and exclusion criteria. After the full-text screening, 9 articles were removed as they did not contain quantitative data, did not estimate the association between socioeconomic inequality and reproductive health care service use with the concentration index, or met the exclusion criteria. A study that did not contain adequate information to match survey year and reproductive health care service analyzed, was also excluded. The remaining 22 articles were included in our quantitative analysis. The 22 studies contain 305 estimates of the concentration index for different countries, different years, and different reproductive health care services. The effect size that we investigate in the quantitative analysis is the inequality in the access or use of reproductive health care services quantified by the Fisher's $z$ transformed concentration coefficient (see Methods section). Details about the publications reviewed can be found in Appendix A2.

\subsubsection{Study characteristics}

Table 2.1 presents the overall study characteristics and reproductive health care service grouping information from the 22 articles that were included in the review. Of the studies included, 90\% were published after the 2010 millennium development goal summit, and $42 \%$ analyzed data collected after the summit year. The studies covered 14 countries, 12 of the studies included four Eastern African countries, 8 studies were from five Southern Africa countries, 7 studies were from four Western Africa, and 1 study was from one from Central Africa. Many studies, $77 \%$, examined inequality in reproductive health care services using national-level cross-sectional survey data from the DHS. Other studies used data from cross-sectional surveys from representative communities in Ethiopia data gathered over 2 years (Karim, Tamire, Medhanyie, \& Betemariam, 2015); the Malawi Second Integrated Household Survey (IHS2) of 20042005 (Makoka, 2009); a cross-sectional household survey carried out in 24 districts in Burkina Faso (Mwase et al., 2018); the South Africa national cross-sectional survey conducted in 2012 (Ngandu, Van Malderen, Goga, \& Speybroeck, 2017); the third (2008) and fourth (2012) South African National HIV Prevalence, Incidence, Behaviour, and Communication Surveys (Wabiri et al., 2016). Socioeconomic inequality was measured using the concentration index in all included studies. Using the EPHPP assessment tool, 14 studies were rated as having high quality, 3 as having moderate quality, and 5 as having low quality.

\subsubsection{Reproductive health care service utilization}

Of the 10 categories of reproductive health care services that we distinguished, five categories were relatively large and contributed more than 30 concentration index 
values (see Table 2.2). These five categories were family planning services, contraceptive services, skilled ANC services, components of ANC, and skilled childbirth services. Other categories were unskilled childbirth, four or more ANC visits, inadequate ANC component, postnatal care services, and HIV services.
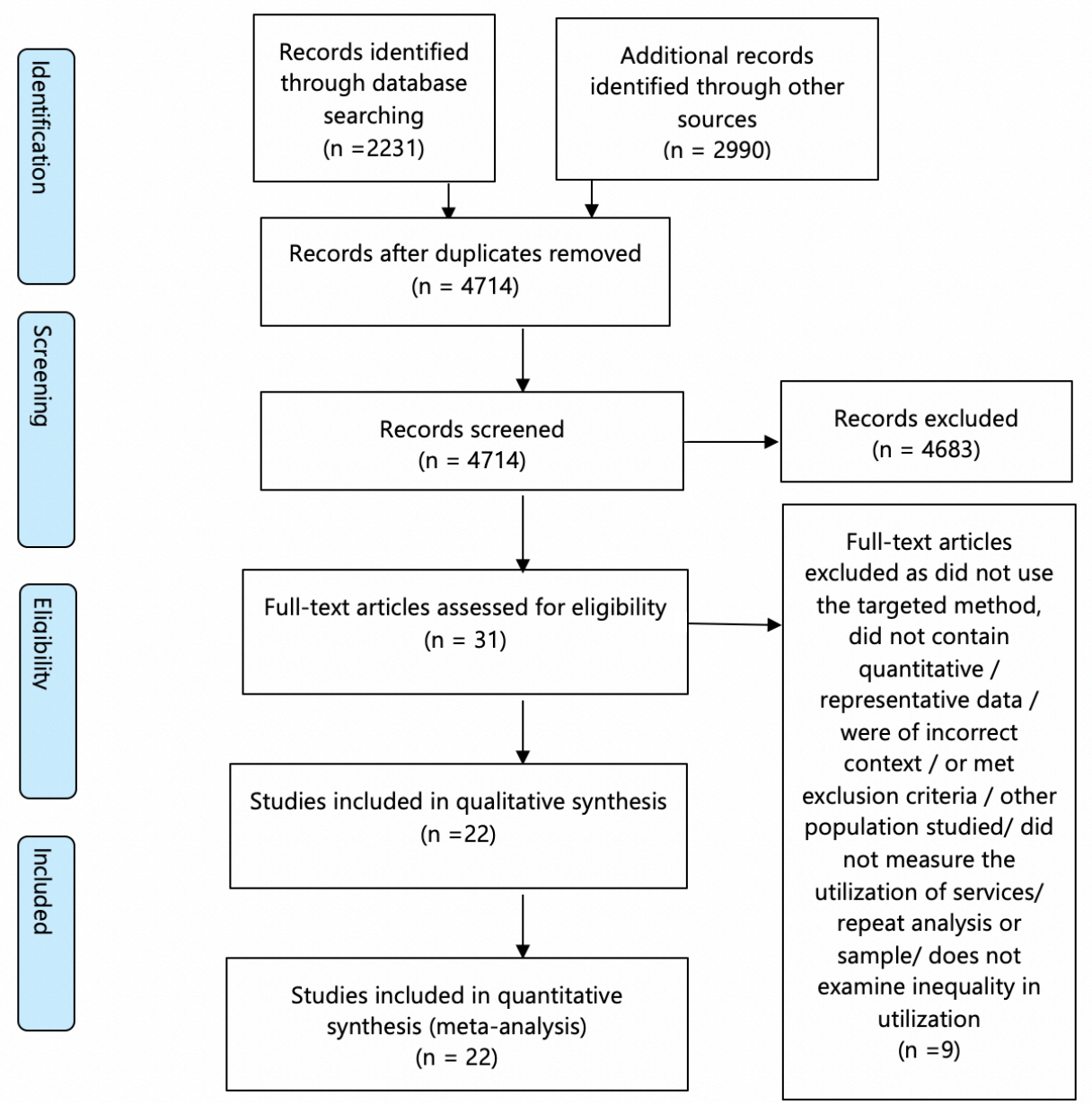

Figure 2.1: PRISMA flow diagram for the systematic review of socioeconomic inequality and reproductive health care services utilization 
Table 2.1: Characteristics of the studies included in the systematic review

\begin{tabular}{|c|c|c|}
\hline $\begin{array}{l}\text { Study } \\
\text { characteristics }\end{array}$ & $\mathrm{N}(\%)$ & Study \\
\hline \multicolumn{3}{|l|}{ Publication year } \\
\hline $2008-2009$ & $2(9.09)$ & Agha \& Do, 2008; Makoka, 2009 \\
\hline $2010-2018$ & 20 (90.91) & $\begin{array}{l}\text { Adeyanju, Tubeuf, \& Ensor, 2017; Alam, Hajizadeh, Dumont, \& Fournier, 2015; } \\
\text { Ambel et al., 2017; Bobo, Yesuf, \& Woldie, 2017; Do, Soelaeman, \& Hotchkiss, } \\
\text { 2015; Gebre, Worku, \& Bukola, 2018; Goli, Nawal, Rammohan, Sekher, \& Singh, } \\
\text { 2018; Jalloh et al., 2019; Karim et al., 2015; Makate \& Makate, 2017; Memirie, } \\
\text { Verguet, Norheim, Levin, \& Johansson, 2016; Mezmur et al., 2017; Mwase et al., } \\
\text { 2018; Ngandu et al., 2017; Obiyan \& Kumar, 2015; Ogundele, Pavlova, \& Groot, } \\
\text { 2018; Onarheim et al., 2015; Van Malderen et al., 2013; Wabiri et al., 2016; Zere } \\
\text { et al., } 2010\end{array}$ \\
\hline
\end{tabular}

Sample year $<=2010$

2011-2016 14 (41.93)

Sample size 0-4000

4001-8000

12 (54.54) Agha \& Do, 2008; Alam et al., 2015; Bobo et al., 2017; Goli et al., 2018; Jalloh et al., 2019; Makate \& Makate, 2017; Makoka, 2009; Mezmur et al., 2017; Mwase et al., 2018; Obiyan \& Kumar, 2015; Ogundele et al., 2018; Onarheim et al., 2015

$>8000$

10 (45.45) Adeyanju et al., 2017; Alam et al., 2015; Gebre et al., 2018; Goli et al., 2018; Jalloh et al., 2019; Memirie et al., 2016; Ngandu et al., 2017; Obiyan \& Kumar, 2015; Ogundele et al., 2018; Onarheim et al., 2015

Data used

DHS $17(77.27)$

Non-DHS
Study quality
high

high

14 (63.64) Adeyanju et al., 2017; Agha \& Do, 2008; Alam et al., 2015; Bobo et al., 2017; Do et al., 2015; Gebre et al., 2018; Goli et al., 2018; Jalloh et al., 2019; Makate \& Makate, 2017; Mezmur et al., 2017; Mwase et al., 2018; Obiyan \& Kumar, 2015; Ogundele et al., 2018; Onarheim et al., 2015

moderate $\quad 3$ (13.64) Memirie et al., 2016; Wabiri et al., 2016; Zere et al., 2010

low $\quad 5(22.73)$

Region $^{1}$

Central Africa 1 (4.55)

Western Africa 7 (31.82)

Southern Africa 8 (36.36)

Eastern Africa

$12(54.55)$ Malderen et al., 2013 et al., 2019. Makate \& Makate, 2017: Memirie et al, 2016: Mezmur et al., 2017. Obiyan \& Kumar, 2015; Ogundele et al., 2018; Onarheim et al., 2015; Van Malderen et al., 2013; Zere et al., 2010

Karim et al., 2015; Makoka, 2009; Mwase et al., 2018; Ngandu et al., 2017; Wabiri et al., 2016

\section{Alam et al., 2015}

Adeyanju et al., 2017; Agha \& Do, 2008; Do et al., 2015; Jalloh et al., 2019;

Mwase et al., 2018; Obiyan \& Kumar, 2015; Ogundele et al., 2018

Alam et al., 2015; Makate \& Makate, 2017; Makoka, 2009; Ngandu et al., 2017;

Wabiri et al., 2016; Zere et al., 2010

al., 2015; Gebre et al., 2018; Goli et al., 2018; Karim et al., 2015; Memirie et al., 2016; Mezmur et al., 2017; Onarheim et al., 2015; Van Malderen et al., 2013

${ }^{1}$ Note that some studies included more than one country and therefore the count $(n=29)$ is greater than the number of studies included $(n=22)$ 


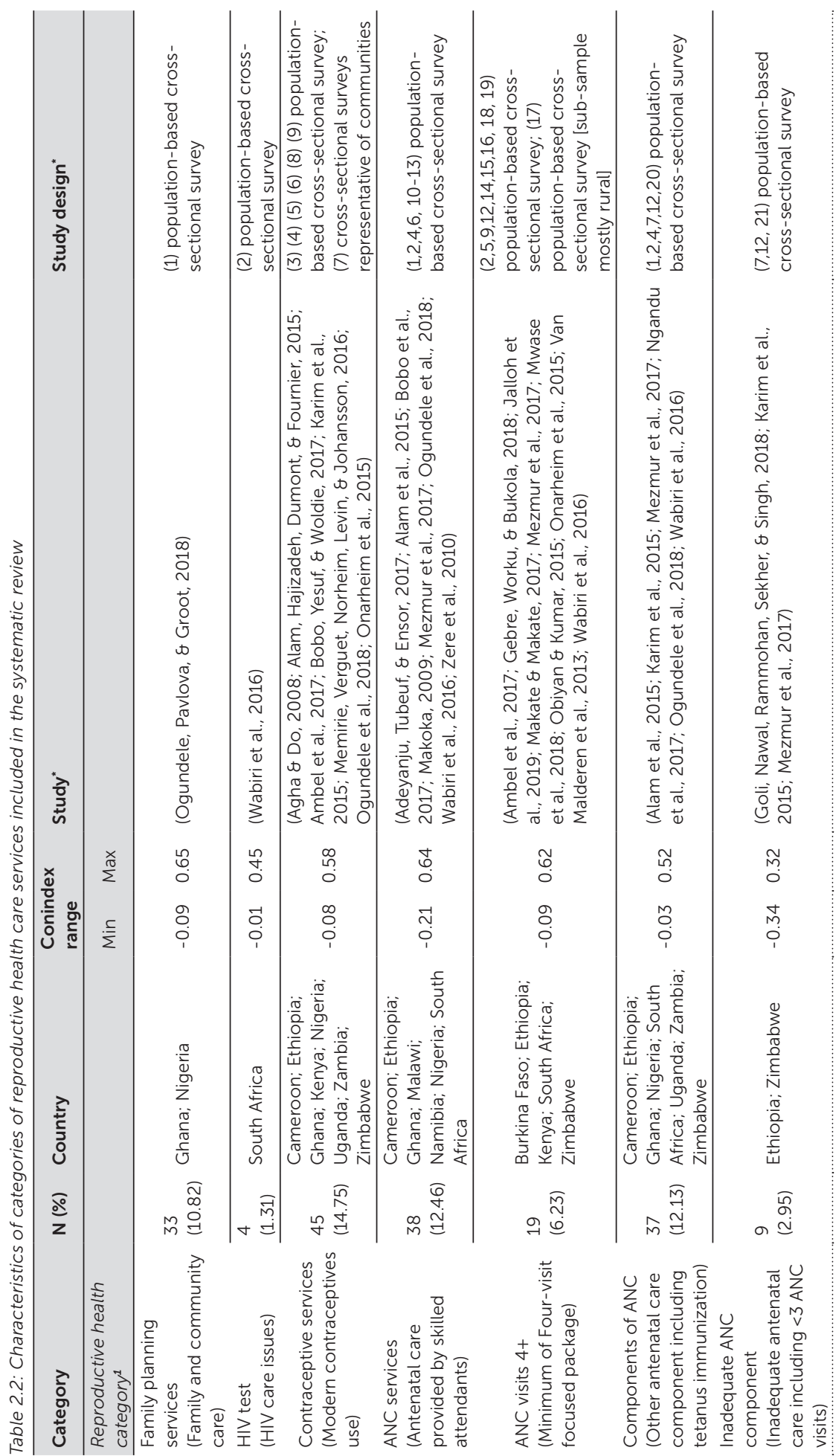




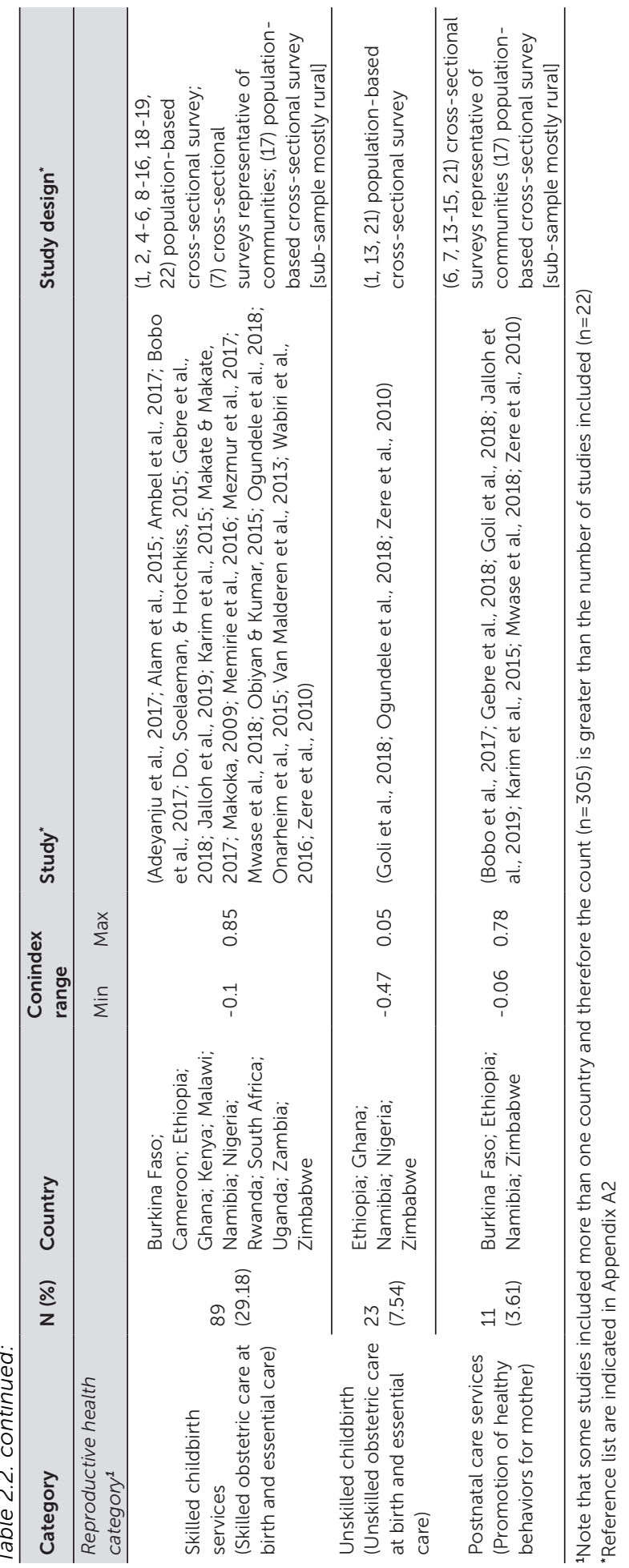




\subsubsection{Meta-analysis}

The 22 studies included in the review contained a total of 305 estimates of the concentration index. The effect size that we report in Table 2.3 is the concentration index back-transformed from Fisher's $z$ unit (see Methods section). Table 2.3 presents further descriptive statistics of the metadata. Specifically, next to the back-transformed concentration indices, Table 2.3 shows a summary of the results in Fisher's $z$ units. The size of the metadata included in each reproductive health category differs. The forest plots of Fisher's $z$ units for socioeconomic inequality and the categories of reproductive health care services are shown in Appendix A3.

Table 2.3: Results of a meta-analysis of reproductive health care services

\begin{tabular}{|c|c|c|c|c|c|c|c|c|c|}
\hline \multirow[b]{2}{*}{ Reproductive health group } & \multirow[b]{2}{*}{$N$} & \multirow{2}{*}{$\begin{array}{l}\text { Effect size } \\
\text { (Conindex) }\end{array}$} & \multirow{2}{*}{$\begin{array}{l}\text { Effect size } \\
\text { (Fisher's z) }\end{array}$} & \multicolumn{2}{|l|}{$95 \% \mathrm{Cl}$} & \multirow{2}{*}{$1^{2} \%$} & \multirow{2}{*}{$\mathrm{Tau}^{2}$} & \multicolumn{2}{|c|}{ Test of $E S=0$} \\
\hline & & & & $\mathrm{LL}$ & UL & & & $z$ & $p$-value \\
\hline All reproductive care services & 305 & 0.202 & 0.205 & 0.174 & 0.236 & 99.80 & 0.075 & 13.010 & 0.000 \\
\hline Family planning services & 30 & 0.268 & 0.275 & 0.178 & 0.373 & 99.80 & 0.074 & 5.540 & 0.000 \\
\hline Contraceptive services & 45 & 0.203 & 0.206 & 0.150 & 0.261 & 99.50 & 0.036 & 7.270 & 0.000 \\
\hline Components of ANC & 37 & 0.142 & 0.143 & 0.089 & 0.197 & 98.50 & 0.009 & 5.220 & 0.000 \\
\hline ANC services & 38 & 0.164 & 0.166 & 0.107 & 0.224 & 99.60 & 0.034 & 5.550 & 0.000 \\
\hline Skilled childbirth services & 89 & 0.343 & 0.358 & 0.295 & 0.422 & 99.80 & 0.093 & 11.040 & 0.000 \\
\hline HIV services & 4 & 0.119 & 0.120 & -0.134 & 0.374 & 98.90 & 0.066 & 0.920 & 0.355 \\
\hline ANC visits $4+$ & 19 & 0.195 & 0.198 & 0.071 & 0.324 & 99.80 & 0.080 & 3.060 & 0.002 \\
\hline Inadequate ANC component & 9 & 0.056 & 0.056 & -0.021 & 0.133 & 99.40 & 0.025 & 0.840 & 0.399 \\
\hline Unskilled childbirth & 23 & -0.201 & -0.204 & -0.245 & -0.164 & 98.50 & 0.009 & 9.900 & 0.000 \\
\hline Postnatal care services & 11 & 0.140 & 0.141 & 0.035 & 0.248 & 99.60 & 0.032 & 2.590 & 0.009 \\
\hline
\end{tabular}

A key observation is the wide range of the summary effect size estimates, as shown in Table 2.3. The average concentration index for family planning services was 0.268 and for contraceptive services it was 0.203 . Both demonstrated inequality detrimental to the population with lower socioeconomic status. For components of ANC and adequate ANC services, the average effect sizes were 0.142 and 0.164 respectively, comparatively lower than the estimates for the other reproductive health care service categories. Particularly, for skilled childbirth services, the observed average concentration index was 0.343 . The pooled concentration index size of all categories, that is, for all reproductive health care services, was 0.202 using 305 observations. All average effect sizes were statistically significant $(p<0.05)$ and suggested that inequalities in the use of reproductive health care services are to the detriment of the poorer population.

Observed $p$-values of associated $Q$ statistics of the reproductive health categories were highly significant and indicated that the true effect differs across studies. Further, 
observed heterogeneity between studies was high in all service categories $\left(I^{2} \geqslant 98.50 \%\right)$. Estimates of between-study variance (T2) were between 0.009 (components of ANC) and 0.096 (skilled childbirth services). Explanations for the observed heterogeneity included potential publication bias, the use of absolute and relative measures of the concentration indices, and the diversity in the outcome measures.

\section{Publication bias}

We explore publication bias in the reproductive health care services that have more than 30 concentration index values, i.e. family planning services, contraceptive services, skilled ANC services, components of ANC, and skilled childbirth services. The funnel plots of the reproductive health care services were asymmetrical, indicating that publication bias was likely. It should be noted however that studies displayed in the plots did not always estimate the same outcome. Specifically, ANC care components included studies that measured if women received any ANC, had an early uptake of HIV testing; inadequate ANC component included less than three ANC, a minimum of one ANC, professional childbirth care included use of institutional childbirth service, medically attended deliveries and skilled birth attendance; contraceptives services also included if a woman currently uses any contraceptive method, family planning method, the level of current modern method. Results from the Egger tests for asymmetry indicated that only the components of ANC category were significant ( $p=$ 0.014), which also indicated possible publication bias. The estimate of all reproductive health care services was however insignificant ( $p=0.104)$.

\section{Meta-regression}

We used random-effects meta-regression to account for the heterogeneity in the estimates of the outcome. Specifically, we used the pooled summary statistic of the reproductive health care services, namely the concentration index in Fisher's $z$ units, as the dependent variable, and various characteristics as independent (explanatory) variables. We estimated three random-effects regression models by adding studylevel covariates for reproductive health categories, study characteristics and countries respectively. Table 2.4 shows the results of the meta-regression for Models 1, 2 and 3 (Fisher's $z$ transformed). The proportion of the explained heterogeneity, accounted for by the covariates of reproductive health care categories included (see adjusted R-squared), was $23.30 \%$ in Model 1. In Model 2, where study characteristics were added, the explained between-study variance increased to $27.87 \%$, and in Model 3 , which included country covariates, it increased to $37.38 \%$. These values indicate that $23.30 \%$ of study heterogeneity may be attributed to the variation in services type, i.e. inequality in utilization varied, depending on the reproductive health care services. 
Also, about $9.51 \%(37.38 \%-27.87 \%)$ of the heterogeneity may be attributed to the contextual differences among countries included. The remaining between-study variance explained by the study characteristics is small at $4.7 \%(27.87-23.30 \%)$ ). 


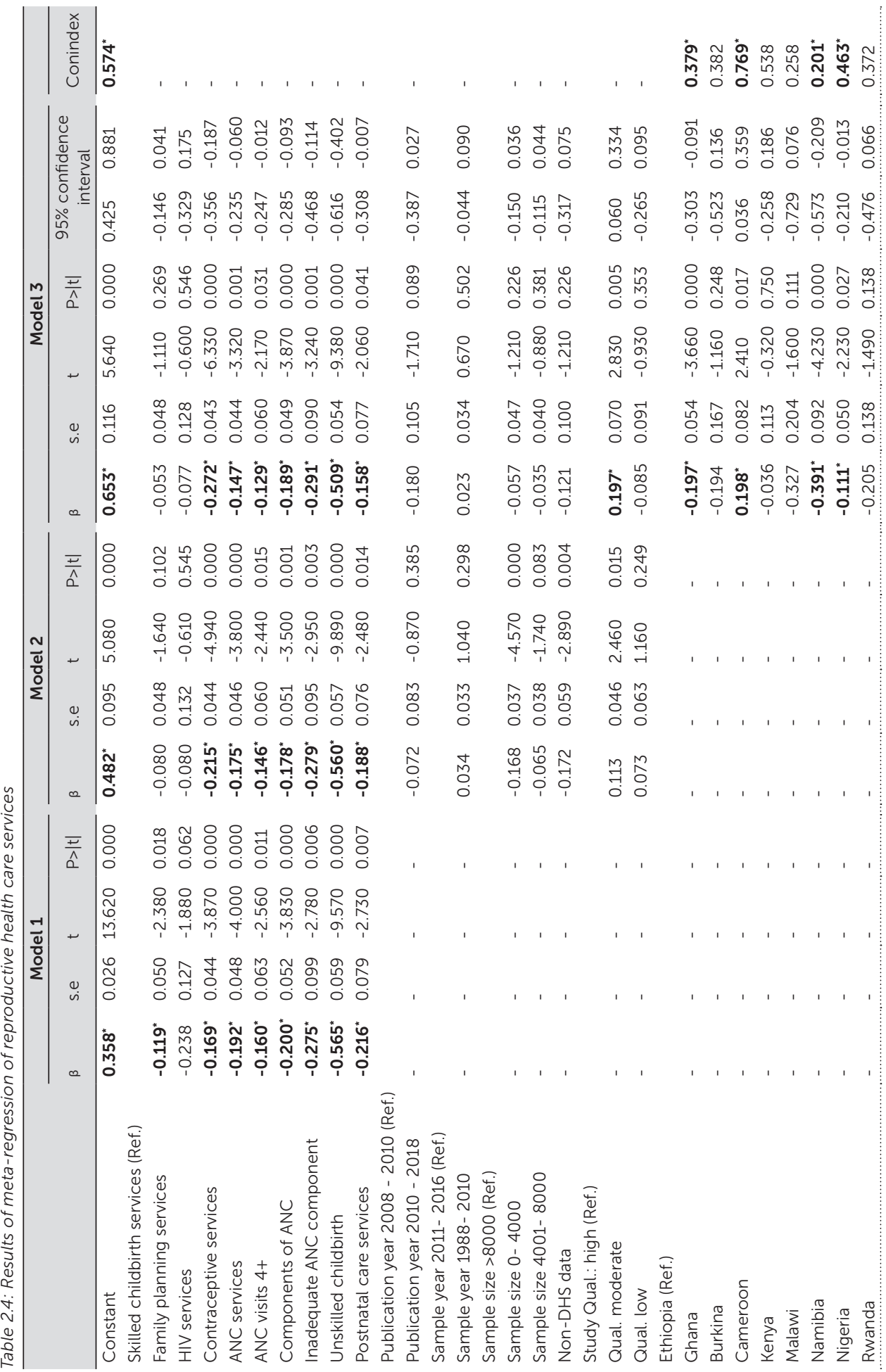




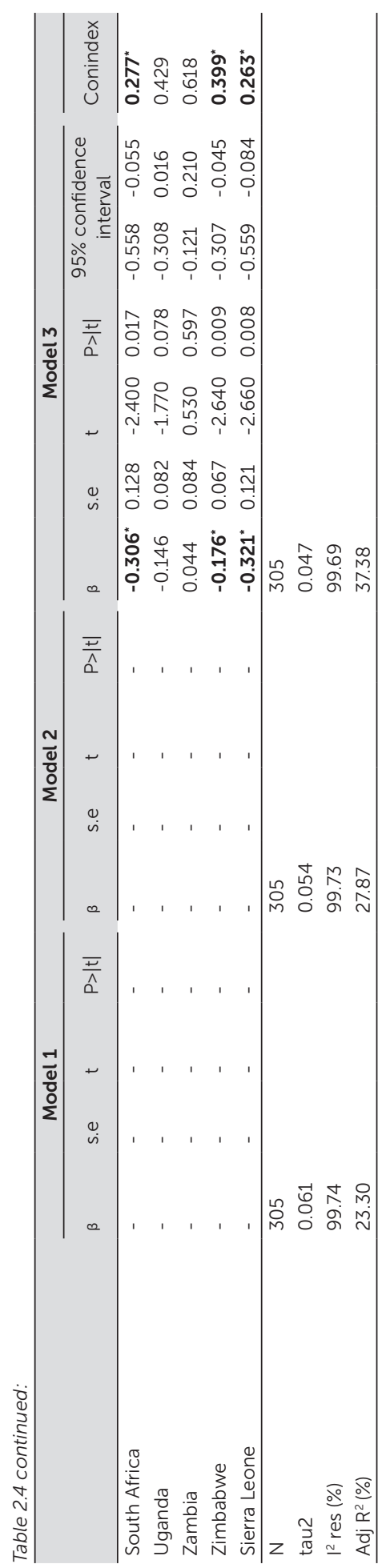


In Table 2.5, Model 3, we controlled for reproductive health categories, study characteristics, and countries. The slope of the model, in Fisher's $z$ unit, was statistically significant and positive $(\beta($ s.e. $)=0.653(0.116), 95 \% \mathrm{Cl}$ : 0.425 to 0.881$)$. This underlines that reproductive health care use increases with socioeconomic status. Statistically significant reproductive health care services categories are contraceptive services, ANC services, four or more ANC visits, components of ANC, and postnatal care. Besides, the strength of the association of inadequate ANC component and unskilled childbirth varied markedly but were both negative $(\beta($ s.e. $)=-0.291(0.090), 95 \% \mathrm{Cl}$ : -0.468 to -0.114$)$ and $(\beta($ s.e. $)=-0.509(0.054), 95 \% \mathrm{Cl}$ : -0.616 to -0.402$)$ respectively, indicating predominance among the poor. The result for moderate quality studies was significant only in Model 3 revealing a weak association. Further, the country dummies of Cameroon, Nigeria, Zimbabwe, Ghana, South Africa, and Namibia were statistically significant and have negative effects on reproductive health care service use compared to Ethiopia except for Cameroon ( $\beta$ (s.e.) $=0.198$ (0.082), 95\% Cl: 0.036 to 0.359). This implies that these countries have less inequality than Ethiopia but not Cameroon.

Post-estimation results based on the results of Model 3 (Table 2.5) show the crossnational differences in the magnitude of inequality across service categories in the concentration index values. For skilled childbirth services across countries, the largest average inequality was 0.692 in Cameroon and the lowest was 0.256 in Namibia. Cameroon, Ethiopia, Nigeria, Ghana, and Zimbabwe have similarly high average inequality in reproductive health care service categories. While South Africa, Sierra Leone, and Namibia have lower pro-wealthy inequality - the latter being the lowest. The high average observed in Cameroon and low average in Namibia is observed in the data for all reproductive health care categories. Namibia has the largest average pro-poor inequality in unskilled childbirth; other countries with high inequality are Zimbabwe, Ghana, and South Africa. Across services, inadequate ANC was concentrated among the poor only in Namibia. Unskilled childbirth is concentrated among the poor in Zimbabwe, Ghana, South Africa, Sierra Leone, and Namibia. 
Table 2.5: Post-estimation of meta-regression in concentration indices

\begin{tabular}{|c|c|c|c|c|c|c|c|c|c|c|}
\hline & 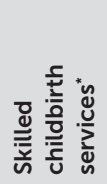 & 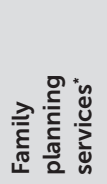 & 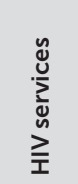 & 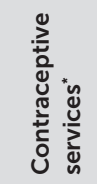 & 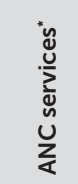 & $\begin{array}{l}+ \\
+ \\
+ \\
\frac{n}{n} \\
\frac{n}{5} \\
u \\
z \\
<\end{array}$ & 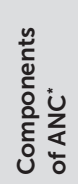 & 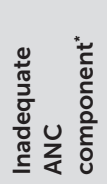 & 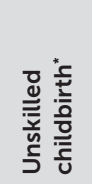 & 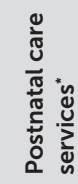 \\
\hline Ethiopia* & 0.574 & 0.537 & 0.52 & 0.364 & 0.467 & 0.481 & 0.433 & 0.347 & 0.143 & 0.458 \\
\hline Ghana* & 0.427 & 0.383 & 0.362 & 0.182 & 0.300 & 0.316 & 0.261 & 0.164 & -0.053 & 0.289 \\
\hline Burkina & 0.429 & 0.385 & 0.364 & 0.185 & 0.302 & 0.319 & 0.264 & 0.166 & -0.050 & 0.292 \\
\hline Cameroon* & 0.692 & 0.663 & 0.649 & 0.522 & 0.607 & 0.618 & 0.580 & 0.508 & 0.329 & 0.600 \\
\hline Kenya & 0.549 & 0.511 & 0.493 & 0.332 & 0.438 & 0.453 & 0.404 & 0.315 & 0.108 & 0.429 \\
\hline Malawi & 0.315 & 0.266 & 0.244 & 0.054 & 0.177 & 0.194 & 0.136 & 0.035 & -0.181 & 0.166 \\
\hline Namibia* & 0.256 & 0.206 & 0.183 & -0.010 & 0.114 & 0.132 & 0.073 & -0.029 & -0.242 & 0.104 \\
\hline Nigeria* & 0.495 & 0.453 & 0.434 & 0.264 & 0.376 & 0.391 & 0.339 & 0.246 & 0.033 & 0.366 \\
\hline Rwanda & 0.420 & 0.376 & 0.355 & 0.174 & 0.292 & 0.309 & 0.253 & 0.156 & -0.061 & 0.282 \\
\hline South Africa* & 0.334 & 0.286 & 0.264 & 0.075 & 0.197 & 0.215 & 0.157 & 0.056 & -0.161 & 0.187 \\
\hline Uganda & 0.468 & 0.425 & 0.405 & 0.231 & 0.345 & 0.361 & 0.308 & 0.213 & -0.002 & 0.335 \\
\hline Zambia & 0.602 & 0.568 & 0.551 & 0.401 & 0.501 & 0.514 & 0.468 & 0.385 & 0.186 & 0.492 \\
\hline Zimbabwe* $^{*}$ & 0.444 & 0.400 & 0.380 & 0.202 & 0.319 & 0.335 & 0.280 & 0.184 & -0.032 & 0.309 \\
\hline Sierra Leone* & 0.320 & 0.272 & 0.250 & 0.060 & 0.183 & 0.200 & 0.142 & 0.041 & -0.175 & 0.172 \\
\hline
\end{tabular}

*Significant covariates from meta-regression

\subsection{DISCUSSION}

The purpose of this study was to systematically review the evidence on the degree of socioeconomic inequality of reproductive health care services utilization in SubSaharan Africa and identify the variance in these inequalities. The results of the metaanalysis indicated that socioeconomic inequality in the use of reproductive health care services is present to the detriment of poorer women. The overall average inequality in reproductive health care services utilization was 0.202 . This aggregated magnitude was much lower compared to some service-specific values, e.g. for family planning services, contraceptive services, skilled ANC services, components of ANC, and skilled childbirth services. Other categories with inequality less than average are HIV services, four or more ANC visits, and postnatal care services. Nevertheless, the presence of an association of socioeconomic inequality with reproductive health care utilization, which is pro-wealthy, is confirmed. This implies that the use of reproductive health care services is unequally distributed to the disadvantage of women in households with low socioeconomic status.

The most inequitable service, compared to other categories, was the utilization of skilled childbirth services, in favor of women with better socioeconomic status - 
usually wealthier households. This could be a result of the emphasis on reducing the high rates of maternal mortality by focusing interventions on access to skilled care during childbirth. These, interventions that mostly address the removal of user fees for facility childbirth services, have not been scaled-up to eliminate out of pocket expenditure for pre-pregnancy, pregnancy and post-natal care (Richard et al., 2013; Ridde et al., 2012). These policies have not eliminated catastrophic costs associated with delivery service use (Ridde et al., 2015). Moreover, interventions in Sub-Saharan Africa have primarily focused on supply-side factors like increasing the coverage of medical doctor, nurse or midwife-assisted childbirth services and their ability to provide obstetric care in health care facilities (Koblinsky et al., 2006; Lassi, Salam, Das, \& Bhutta, 2014). However, improved coverage does not necessarily imply increased utilization, or vice versa, and could increase inequality. The relatively medium coverage in Ghana (Odeyemi \& Nixon, 2013), and the larger coverage in Namibia, of skilled attendance at birth and delivery in a health facility (Zere, Kirigia, Duale, \& Akazili, 2012) resulted in pro-wealthy inequality. This is confirmed by a study that showed that women in the poorest wealth group remain unlikely to have facility childbirths despite increased coverage brought about by the fee exemption policies in Ghana, Senegal and Sierra Leone (McKinnon et al., 2015). Removing user fees may increase utilization but will not eliminate inequality in utilization if the coverage of services is disproportionately spread to the disadvantage of less wealthy women.

The average size of the pro-wealthy inequality was higher for family planning services, contraceptive services compared with skilled ANC services, and adequate components of ANC. The provision of modern family planning services, sometimes marginally subsidized, mostly relies on public sector funding and provision in most African countries. The government initiatives are rarely available, inconsistent, and are often poorly applied with limited effects. Donor funding for family planning as a proportion of other reproductive health aid is meager even though access significantly reduces maternal mortality (Hsu et al., 2013). This could partly account for inequalities in resources available for family planning services. Besides, another study showed that free health care policies in rural Burkina Faso have little impact on women's contraception use, in part, due to economic barriers and limited autonomy (Samb \& Ridde, 2018). Hosseinpoor et al. (2011) investigated the inequalities in maternal and child health services in selected Sub-Saharan African countries. The authors showed that the national coverage gaps and absolute inequality (percentage points) in family planning were, respectively, 64\% and 27 in Burkina Faso, 51\% and 17 in Ghana, 59\% and 18 in Cameroon, 73\% and 19 in Senegal, 77\% and 20 in Sierra Leone, 17\% and 8 in Zimbabwe. The findings suggest that low coverage can be evidence of higher pro- 
wealthy inequality. Similarly, in countries including Ethiopia, Nigeria, Kenya, Ghana and Zimbabwe, identified as priority countries for maternal, newborn and child survival initiatives, little improvements in the coverage gap of family planning are observed (Barros et al., 2012; Countdown Equity Analysis et al., 2008). This is indicative of the low coverage of family planning services among socioeconomically disadvantaged women. Nonetheless, increased coverage of services does not preclude inequalities though it can lessen it.

Fostering access and utilization among specific socioeconomic groups could help reduce inequality and increase access to family planning services. A study noted that although increased availability of modern contraceptives contributed to more women in Ghana being able to afford modern contraceptives which increased use, this improvement was unequally spread across socioeconomic groups (Asamoah, Agardh, \& Ostergren, 2013). In a multi-country study, the payment for modern contraceptives was not a barrier to utilization among women in Kenya (Asaolu, Nuno, Ernst, Taren, \& Ehiri, 2019). Overall, the supply-side interventions do not appear to appropriately capture the differentials in care needs, or in the ability to overcome use barriers.

Comparatively, national initiatives exist to provide basic maternal and child health services using insurance schemes, community-based outreach services (Abimbola et al., 2012; Nyonator et al., 2005; Warenius et al., 2006) and primary health care centers (Onwujekwe et al., 2016). These interventions also promote the uptake of ANC, routine immunization, and iron/folic acid supplementation. Other obstetrics services exist in some countries and benefit from fairly better governmental support. However, these policies have significant shortcomings - they are not usually nationwide implemented, or where payments exclusion are in place, do not target vulnerable groups, thus, benefiting the better-off population. For example, Namibia appears to have moderately fair and increasing coverage (Bonfrer et al., 2014). More involvement of the public sector and the scale-up of family planning services could adequately reduce inequalities by better reaching vulnerable populations.

In addition, socioeconomically disadvantaged women have used pre-pregnancy services such as HIV testing, family planning information and modern contraceptives even less. Our results are consistent with previous studies that pre-pregnancy and pregnancy care services constitute a larger burden for women in all socioeconomic status, but are better accessed by women in wealthier households (Arthur, 2012; Fagbamigbe \& Idemudia, 2015; Johnson et al., 2016). 
Although the average size of inequality is lower for skilled ANC services and adequate components of ANC, it does not suggest that the coverage of these services is higher. Barros et al. (2012) in an analysis of equity in maternal health interventions in high maternal or child mortality countries noted that moderate levels of inequality in the use of ANC with a skilled provider exist despite higher coverage observed. Few of the fee exemptions for maternal care policies in Sub-Saharan African countries include coverage from pre-pregnancy through pregnancy care and fewer have eligibility criteria to address individual social determinants of health (Hatt et al., 2013; Richard et al., 2013). What is clear is the low coverage and inequality in family planning and other reproductive health care services. Moreover, these inequalities are higher in countries without any form of user fee exemption - Cameroon, Nigeria, and highest where the coverage of maternity services is extremely low - Ethiopia. A resolution to this issue would be the provision of user fees exemptions and a scale-up in countries where sexual and reproductive health care needs are not covered or the poorest are not targeted.

Evidence of considerable between-study heterogeneity was found in our meta-analysis. Heterogeneity between studies is not unexpected given the diversity in the sample design, outcome variables and populations examined in the analysis. The heterogeneity explained increased when covariates for reproductive health care categories, study characteristics, and countries were included. Partially, the categories family planning services, contraceptive services, skilled antenatal services, ANC visits 4+, components of ANC, inadequate antenatal component, unskilled childbirth, and postnatal care services explained the heterogeneity found. Moderate quality studies explained part of the heterogeneity. This means that moderate-quality cross-sectional studies might limit the conclusion, but not studies with a low-quality score index. Ghana, Cameroon, Namibia, Nigeria, South Africa, and Zimbabwe further contributed to the differences across countries. Estimates were higher in Cameroon, Nigeria, Zimbabwe, and Ghana and lower in Namibia, Sierra Leone, and South Africa. Our findings may reflect a true trend in inequality in reproductive health care across countries (Hosseinpoor et al., 2011). Alternatively, this maybe is due to the study methodology. The generalizability of the findings of this study should be done with care as a result of potential publication bias arising from the absence of publications from the French and Portuguesespeaking countries in Sub-Saharan Africa. The amount of data varied across countries, which may have reduced the representativeness of some samples (Simon, Goldberg, Von Korff, \& Ustun, 2002). It may otherwise be due to measurement bias that we were unable to control for. We recommend that future studies explicitly report whether absolute and relative measures of inequality are used. 


\section{Study limitation}

The studies included in the meta-analysis reported absolute and relative measures of inequality. It could be expected that the types of measures used in the studies have had a weighted effect on the summary estimates (Clarke et al., 2002). In addition, we only include published work from three databases, which could have introduced publication bias in the review. We also only cover English-language publications, which

means that we might have missed relevant evidence reported in another language. Absolute, compared with relative concentration indices report lower magnitudes of inequality (Clarke et al., 2002). As a result, outcomes in the meta-analysis reporting more relative concentration indices would have higher magnitudes of inequality. However, the statistical methods employed in the studies reviewed are similar, which facilitates an overall cross-study comparison of inequalities in reproductive health care use (Rosenblad, 2009). This, in addition to using the most representative findings from primary studies, systematic quality assessment of primary studies, and the adjusted measures of health inequalities, improved the validity of our meta-analysis (FinfgeldConnett, 2010). We acknowledge that the coverage gap that reflects the availability of needed health care services is important to reflect the utilization pattern. However, about half of the studies reviewed do not contain data on coverage of reproductive health care services. Though redundant data were carefully screened for, and removed as much as possible, data from different studies may overlap since different publications use the same dataset but construct the reproductive health outcomes differently. Lastly, our meta-analysis demonstrated high heterogeneity related to the grouping of various reproductive health care services that could otherwise be categorized differently. Future research that examines socioeconomic inequality and reproductive health can include studies from more journals and perform subgroup analyses with studies that employ similar designs and samples in order to address heterogeneity aptly.

\subsection{CONCLUSION}

A clear deduction of this review is that the focus on skilled childbirth services through user fees removal has led to persisting inequality. In Sub-Saharan African countries, skilled childbirth services are better used in countries where there are user fee policies that reduce or eliminate the financial burden for professional childbirth services. This shows that fee-waiver policies improve the use of services and with better targeting, such policies have the potential to address inequalities. Without appropriate resource allocation, women with low socioeconomic status who have persistently low use of all reproductive health care services due to peculiar characteristics and indirect costs will 
continue to patronize unskilled reproductive providers for health care. Nevertheless, implementing universal health coverage measures should in tandem address all dimensions of the continuum of care: pregnancy, childbirth, and the postnatal periods. Furthermore, examining the magnitude of inequality in parallel with coverage information on specific reproductive health care services will better indicate where context-specific interventions are of importance. User fee policies for reproductive health care need to be broadened to accommodate pre-pregnancy and pregnancy services not yet covered. Lastly, cross-national differences in reproductive health care services, indicate countries where inequalities are most pronounced in use. Although there has been an increase in the interest in the quantitative measurement of inequalities after the MDGs, less than $50 \%$ are empirical studies.

We were able to identify gaps in the literature that need further consideration. There were very few alternative data sources and very few country-based studies. Additional research on inequalities in family planning and maternal care services is needed across Sub-Saharan Africa countries. This should provide adequate sample-sizes for further analysis. We were also unable to assess the effect of the country on inequality across reproductive health care. More cross-national studies and divergent datasets will be useful for stronger conclusions. Further investigation of these limitations is required for a clearer understanding of context-specific inequalities in reproductive health care services across Sub-Saharan Africa. 
INEQUALITIES IN REPRODUCTIVE HEALTH CARE SERVICES ACROSS SUB-SAHARAN AFRICA. A SYSTEMATIC REVIEW AND META-ANALYSIS 



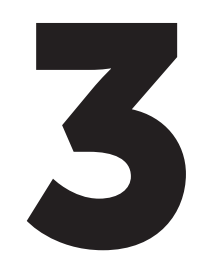

\section{PATTERNS OF ACCESS TO REPRODUCTIVE HEALTH CARE SERVICES IN GHANA AND NIGERIA: RESULTS OF A CLUSTER ANALYSIS}

\section{Draws upon:}

Ogundele, O. J., Pavlova, M., \& Groot, W. (2020). Patterns of Access to Reproductive Health care services in Ghana and Nigeria: Results of a Cluster Analysis. BMC Public Health, 20(1), 549. 


\section{ABSTRACT}

\section{Background}

Inequalities in access to health care result in systematic health differences between social groups. Interventions to improve health do not always consider these inequalities. To examine differences in access to reproductive health care services, the use of family planning and maternal care by women in Ghana and Nigeria is explored.

\section{Methods}

We used population-level data from the Ghana and Nigeria Demographic Health Surveys of 2014 and 2013 respectively. We applied a two-step cluster analysis followed by multinomial logistic regression analysis.

\section{Results}

The initial two-step cluster analyses related to family planning identified three clusters of women in Ghana and Nigeria: women with high, medium and poor access to family planning services. The subsequent two-step cluster analyses related to maternal care identified five distinct clusters: higher, high, medium, low and poor access to maternal health services in Ghana and Nigeria. Multinomial logistic regression showed that compared to women with secondary/higher education, women without education have higher odds of poor access to family planning services in Nigeria (OR=2.54, 95\% $\mathrm{Cl}$ : 1.90-3.39) and in Ghana (OR=1.257, 95\% Cl: 0.77-2.03). Compared to white-collar workers, women who are not working have increased odds of poor access to maternal health services in Nigeria (OR=1.579, 95\% Cl: 1.081-2.307, $p \leq 0.01)$. This association is not observed for Ghana. Household wealth is strongly associated with access to family planning services and maternal health care services in Nigeria. Not having insurance in Ghana is associated with low access to family planning services, while this is not the case in Nigeria. In both countries, the absence of insurance is associated with poor access to maternal health services.

\section{Conclusion}

These differences confirm the importance of a focused context-specific approach towards reproductive health care services, particularly to reduce inequality in access resulting from socio-economic status. Interventions should be focused on the categorization of services and population groups into priority classes based on needs assessment. In this way, they can help expand coverage of quality services bottom up to improve access among these vulnerable groups. 


\subsection{BACKGROUND}

Inequalities in access to health care can result in health differences between social groups. Interventions to create universal access to health care and to improve health outcomes do not always consider these inequalities. Women are exposed to unequal access to health care services globally (Cook, 1993). This is particularly the case for reproductive health care services (Braveman \& Tarimo, 2002), which include contraceptives, maternal health services, and services related to sexual health (Canning \& Schultz, 2012; Gavin et al., 2014; Koblinsky, Campbell, \& Heichelheim, 1999). Targets have been set to improve these services. For example, the Sustainable Development Goal 3 aims to ensure universal access to reproductive health care services (United Nations, 2015).

As outlined in Chapter 1, factors that determine access to reproductive health care services are related to both demand and supply and can be divided into social and economic factors (Gulliford et al., 2002). Education, occupation, wealth and possession of insurance among others are significant predictors of inequality in access to reproductive health care services in Sub Saharan Africa (Ayanore, Pavlova, \& Groot, 2016b; Darroch \& Singh, 2013; Dong, Kouyate, Cairns, \& Sauerborn, 2005; Kabir, Iliyasu, Abubakar, \& Asani, 2005; Overbosch, Nsowah-Nuamah, van den Boom, \& Damnyag, 2004; Solar \& Irwin, 2010). However, previous studies have mostly focused on the determinants of service use in a country or region (Addai, 2000; Babalola \& Fatusi, 2009; Overbosch et al., 2004). There is a need for cross-country comparisons to shed light on similarities and/or dissimilarities between groups of users of reproductive health care services in Sub-Saharan African countries.

This study examines access to reproductive health care services among women of reproductive age in Ghana and Nigeria. We use data from the DHS of Ghana carried out in 2014 and that of Nigeria carried out in 2013. The two countries are selected for this study based on the similarities in trends, health outcomes as well as data availability (see Chapter 1). At the same time, their health care systems are different. For example, Ghana has an established national health insurance system, while there is no such well-established system in Nigeria (Dixon et al., 2013). Ghana and Nigeria introduced a minimal user fee in the early 1970s which was later abandoned in both countries due to cash crunches (Ibiwoye \& Adeleke, 2008). Ghana offers free-of-charge maternal care and the health insurance scheme in the country is reported to cover $65 \%$ of the population which reduces the out-of-pocket health expenditure $(66 \%$ of total health spending) (Odeyemi \& Nixon, 2013). One study using a cluster analysis method has 
shown that there are differences in the adequacy of maternal care available in Ghana and that there are disparities in the sociodemographic characteristics that determine access (Ayanore et al., 2016b). Insurance in Nigeria covers $3.5 \%$ of the population with out-of-pocket health expenditure amounting to over $90 \%$ of total health spending (Ibiwoye \& Adeleke, 2008; Odeyemi \& Nixon, 2013). These differences are expected to result in differences in access to reproductive health care services, which we investigate in this chapter.

\subsection{METHODS}

The DHS are nationally representative cross-sectional surveys carried out in low- and middle-income countries periodically (Ghana Statistical Service, Ghana Health Service, \& ICF International, 2014; NPC \& ICF, 2014). The DHS survey method was described in detail in Chapter 1.

We only used data for women of reproductive age (15-49 years) in Ghana and Nigeria who had given birth during the last 5 years before the survey and were able to provide information on the use of reproductive health care services. The study included 4,142 women from the DHS of Ghana and 7,725 women from the DHS of Nigeria.

We first performed two-step cluster analyses, which provided insight into the patterns of reproductive health care services use among women of reproductive age in both countries. The method of cluster analysis is identified as a favorable way to quantify similarities or dissimilarities based on respondents' data, and to classify respondents into groups based on the available respondents' data (Romesburg, 2004; Schuetz, 2011). The rationale behind the application of cluster analysis method in this study is that women of reproductive ages (15-49) with the same factors influencing their reproductive health services utilization, will report similar pattern which will be a basis for creating cluster groups and group membership. Two-step cluster analysis was preferred over other methods due to the methods scalability, which is more appropriate for the large datasets used in the study (Chiu, Fang, Chen, Wang, \& Jeris, 2001). Also, in contrast to principal component analysis method, a data reduction method, cluster analysis is a group discovery method. Four cluster analyses were carried out, namely one for family planning services and another one for maternal health services for each of the two countries. In particular, the two-step clustering procedure uses the Schwarz's Bayesian Information Criterion (BIC) method to determine the number of clusters. Different clustering solutions are compared and the clustering solution with 
the lowest BIC is selected by the procedure. We inspected this clustering and accept it as adequate. The stability and reliability of the cluster analyses were confirmed by repeating the clustering procedure no less than 10 times. The repeated analysis resulted in the same cluster quality. The two-step cluster analysis procedure specifies the clustering quality based on the Silhouette Index (SI). The SI indicates how well each subject/object lies within its cluster, and thus, it validates the clustering outcomes. The SI ranges from -1 to 1 . SI greater or equal to 0.5 indicates good clustering quality.

We titled the clusters based on the quality and adequacy of medical care used by women in each cluster compared to what is usually provided in government-licensed medical facilities. Thus, in the poor access cluster, on average, women reported using less and lower quality care than the care usually provided at government-licensed facilities, and, in the high access cluster, women reported using more and better care. Details about the cluster composition and structure are presented in Appendix B1.

Multinomial logistic regression was used to identify factors associated with the cluster membership determined during the cluster analyses. A total of four regression analyses were conducted. The cluster membership generated in each cluster analysis was the dependent variable in the multinomial logistic regression analyses. The explanatory variables consisted of women's background characteristics that were found to be associated with the use of family planning services and maternal health services in previous studies and were available in our dataset. Sample weights were applied for the multinomial logistic regression. Software package SPSS version 23 was used for all data analyses.

\subsection{RESULTS}

Descriptive statistics on the socioeconomic characteristics of the two samples and primary results of the two-steps cluster analyses can be found in Appendix B2. Below, we present the key findings of the cluster analyses as well as the results of the regression analyses.

\subsubsection{Cluster analysis}

The two-step cluster analysis of family planning service use in Ghana automatically produced 3 distinct clusters. In the two-step cluster analysis of family planning service use in Nigeria, the number of clusters ( 3 clusters) was fixed in advance to be able to produce meaningful clusters. Figures 3.1 and 3.2 present the quality results of the 
cluster analyses for access to family planning. The two-step cluster analysis for Ghana automatically produced 3 distinct clusters with goodness-of-fit from fair to good (greater or equal 0.5).

Figures 3.3 and 3.4 present the results of the quality of cluster analyses for access to maternal care. Each cluster analysis for Nigeria and Ghana produced a silhouette measure of cohesion and separation from fair to good (figures $6 \& 7$ ). Both two-step cluster analysis for access to maternal health care services automatically produced 5 clusters of women of reproductive ages in Nigeria and Ghana.

The clusters are presented in Table 3.1. The clusters were inspected and labeled as high, medium, and poor access to family planning services based on the services used by women in each cluster (see Methods section).

Model Summary

\begin{tabular}{|l|l|}
\hline Algorithm & TwoStep \\
\hline Inputs & 5 \\
\hline Clusters & 3 \\
\hline
\end{tabular}

Cluster Quality

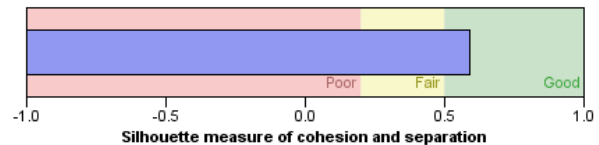

Figure 3.1: Goodness-of-fit of access to family planning services in Nigeria

Model Summary

\begin{tabular}{|l|l|}
\hline Algorithm & TwoStep \\
\hline Inputs 13 \\
\hline Clusters & 5 \\
\hline
\end{tabular}

Cluster Quality

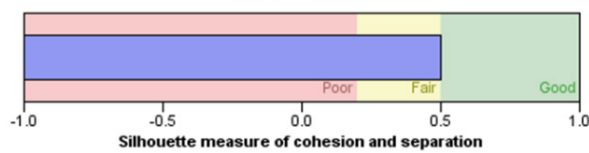

Figure 3.3: Goodness-of-fit of access maternal health care services in Nigeria

\section{Model Summary}

\begin{tabular}{|l|l|}
\hline Algorithm & TwoStep \\
\hline Inputs & 6 \\
\hline Clusters & 3 \\
\hline
\end{tabular}

Cluster Quality

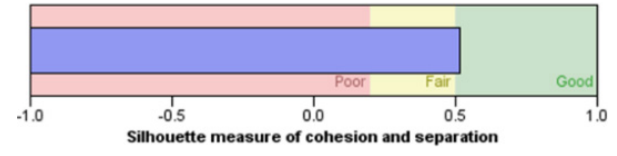

Figure 3.2: Goodness-of-fit of access to family planning services in Ghana

Model Summary

\begin{tabular}{|l|l|}
\hline Algorithm & TwoStep \\
\hline Inputs & 10 \\
\hline Clusters & 5 \\
\hline
\end{tabular}

Cluster Quality

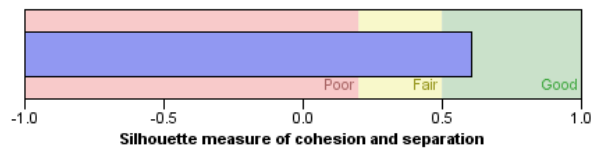

Figure 3.4: Goodness-of-fit of access maternal health care services in Ghana 
Table 3.1: Frequency distribution of cluster membership

\begin{tabular}{|c|c|c|c|c|}
\hline \multirow[b]{3}{*}{ Cluster group } & \multicolumn{4}{|c|}{ Family planning services } \\
\hline & \multicolumn{2}{|c|}{ Ghana } & \multicolumn{2}{|c|}{ Nigeria } \\
\hline & $\mathrm{N}$ & $\%$ & $\mathrm{~N}$ & $\%$ \\
\hline Poor-access & 2755 & 64.2 & 5638 & 71.5 \\
\hline Medium Access & 918 & 14.4 & 1507 & 9.3 \\
\hline \multirow[t]{4}{*}{ High-Access } & 619 & 21.4 & 736 & 19.1 \\
\hline & \multicolumn{4}{|c|}{ Maternal health service } \\
\hline & \multicolumn{2}{|c|}{ Ghana } & \multicolumn{2}{|c|}{ Nigeria } \\
\hline & $\mathrm{N}$ & $\%$ & $\mathrm{~N}$ & $\%$ \\
\hline Low-access & 293 & 7.1 & 346 & 4.4 \\
\hline Poor-Access & 1053 & 25.4 & 1452 & 18.5 \\
\hline Medium-Access & 756 & 18.2 & 2027 & 25.9 \\
\hline High-Access & 952 & 23.0 & 1693 & 21.6 \\
\hline Higher-access & 1092 & 26.3 & 2315 & 29.6 \\
\hline
\end{tabular}

Note: The total percentage of Family planning in Nigeria does not add up to $100 \%$ due to approximation.

In the two-step cluster analyses for Ghana and Nigeria (Table 3.1) regarding family planning services, three distinct clusters are identified for access to these services in each country. The cluster with high access to family planning services captures $19.1 \%$ and $21.4 \%$ of women in Nigeria's and Ghana's sample respectively. The other extreme is the third cluster that consists of women whose access can be described as poor; $71.5 \%$ of women in Nigeria's sample belong to this cluster and $64.2 \%$ of women in Ghana's sample. We did not predefine the number of clusters for maternal health services. For both countries, the two-step cluster analyses of maternal health services resulted in five clusters, which we inspected and labeled as higher, high, medium, low and poor access to maternal health services. The higher-access cluster captures $29.6 \%$ of women in the Nigeria's sample and $26.3 \%$ of the women in Ghana's sample. Relative to the other four clusters, a larger proportion of members of this cluster report that they accessed government hospitals for ANC and used institutional maternal care more. The high-access cluster consists of $21.6 \%$ of women in the Nigeria's sample and 23.0 \% in Ghana's sample. For both countries, this cluster has a lower proportion of women who accessed government health centers for ANC or got assistance from physicians during childbirth. Members of the medium-access cluster in both countries used private facilities for ANC as well as for childbirth. This cluster of women makes up $25.9 \%$ of Nigeria's sample and $18.2 \%$ of the Ghana sample. Members of the lowaccess cluster in both countries mostly are women who report that they accessed government health posts/dispensaries for ANC but did not have skilled assistance during childbirth. In Nigeria's sample, $4.4 \%$ of women fall into this cluster and in the Ghana's sample, this share is $7.1 \%$. Lastly, $18.5 \%$ and $25.4 \%$ of women from the Nigeria's 
and Ghana's sample respectively are members of the poor-access cluster. Members of this cluster mostly did not receive institutionalized maternal care. For both countries, the poor-access cluster has a high proportion of members who had home childbirth and used traditional birth attendants during childbirth.

\subsubsection{Regression analysis}

The dependent variables in the four multinomial logistic regressions were the four cluster membership variables generated in the cluster analyses. Tables 3.2 and 3.3 present the odds ratios for the four regressions. Information about the independent variables used and the full results of the regression analyses can be found in Appendix B3.

For family planning services, the results in Table 3.2 show that in both countries, women with no education, compared to women with secondary or higher education, have higher odds to belong to the poor-access family planning cluster (in Nigeria $\mathrm{OR}=2.544,95 \% \mathrm{Cl}: 1.907-3.395, p \leq 0.01$ and in Ghana $\mathrm{OR}=1.527,95 \% \mathrm{Cl}: 1.173-1.988$, $p \leq 0.01$ ). Increased odds of having poor-access to family planning services are found for women in Ghana who do not belong to white-collar workers but not among women who live in rural areas, and also not among women in any of the wealth quintiles. Higher odds of poor-access to family planning services are also found for women in Nigeria who belong to the service-occupational category (OR=1.283, 95\% $\mathrm{Cl}: 1.002-1.642, p \leq 0.05)$, compared with white-collar workers. The odds of pooraccess are as much as three times higher among the poorest quintile $(O R=3.417,95 \%$ $\mathrm{Cl}: 1.825-6.396, p \leq 0.01)$ than the richest quintile; and among those who have no insurance $(\mathrm{OR}=1.374,95 \% \mathrm{Cl}: 1.011-1.867, p \leq 0.05)$ compared to those with insurance.

Table 3.2: Odds ratio of family planning services. Nigeria and Ghana (multinomial logistic regression)

\begin{tabular}{|c|c|c|c|c|}
\hline \multirow{4}{*}{$\begin{array}{l}\text { Background } \\
\text { characteristics }\end{array}$} & \multicolumn{2}{|l|}{ Nigeria } & \multicolumn{2}{|l|}{ Ghana } \\
\hline & Medium-access & Poor-access & Medium-access & Poor-access \\
\hline & \multicolumn{2}{|c|}{ Reference category: High access } & \multicolumn{2}{|c|}{ Reference category: High access } \\
\hline & Exp B $(95 \% \mathrm{Cl})$ & $\operatorname{Exp} \mathrm{B}(95 \% \mathrm{Cl})$ & Exp B $(95 \% \mathrm{Cl})$ & $\operatorname{Exp} B(95 \% \mathrm{Cl})$ \\
\hline Maternal age & $1.018^{a}(0.998-1.038)$ & $1.015^{\mathrm{b}}(1.001-1.029)$ & $1.047^{c}(1.023-1.072)$ & $1.037^{c}(1.019-1.055)$ \\
\hline $\begin{array}{l}\text { Number of children } \\
\text { alive }\end{array}$ & $0.940^{a}(0.878-1.007)$ & $0.774^{c}(0.738-0.813)$ & $0.851^{\mathrm{c}}(0.777-0.931)$ & $0.849^{c}(0.795-0.908)$ \\
\hline \multicolumn{5}{|l|}{ Marital status } \\
\hline Married (ref) & 1 & 1 & 1 & 1 \\
\hline Never married & $0.338^{c}(0.185-0.619)$ & $0.550^{c}(0.395-0.767)$ & $1.068(0.705-1.617)$ & $1.359^{b}(1.008-1.833)$ \\
\hline $\begin{array}{l}\text { Widowed/separated/ } \\
\text { divorced }\end{array}$ & $1.038(0.606-1.777)$ & $1.531^{\mathrm{b}}(1.052-2.228)$ & $1.004(0.656-1.535)$ & $0.935(0.695-1.258)$ \\
\hline \multicolumn{5}{|l|}{ Maternal Education } \\
\hline Secondary/ Higher (ref) & 1 & 1 & 1 & 1 \\
\hline No education & $1.257(0.776-2.037)$ & $2.544^{c}(1.907-3.395)$ & $1.350(0.941-1.938)$ & $1.527^{c}(1.173-1.988)$ \\
\hline Primary & $0.825(0.642-1.061)$ & $1.111(0.939-1.314)$ & $0.817(0.601-1.112)$ & $0.961(0.774-1.192)$ \\
\hline
\end{tabular}


Table 3.2 continued:

\begin{tabular}{|c|c|c|c|c|}
\hline \multirow{4}{*}{$\begin{array}{l}\text { Background } \\
\text { characteristics }\end{array}$} & \multicolumn{2}{|l|}{ Nigeria } & \multicolumn{2}{|l|}{ Ghana } \\
\hline & Medium-access & Poor-access & Medium-access & Poor-access \\
\hline & \multicolumn{2}{|c|}{ Reference category: High access } & \multicolumn{2}{|c|}{ Reference category: High access } \\
\hline & Exp B (95\% Cl) & Exp B $(95 \% \mathrm{Cl})$ & Exp B $(95 \% \mathrm{Cl})$ & Exp B $(95 \% \mathrm{Cl})$ \\
\hline \multicolumn{5}{|l|}{ Maternal Occupation } \\
\hline White collar (ref) & 1 & 1 & 1 & 1 \\
\hline Not working & $1.006(0.706-1.435)$ & $1.135(0.882-1.459)$ & $1.732^{\mathrm{b}}(1.027-2.921)$ & $2.194^{c}(1.447-3.325)$ \\
\hline Services and manual & $1.134(0.806-1.594)$ & $1.283^{\mathrm{b}}(1.002-1.642)$ & $1.227(0.723-2.081)$ & $1.727^{c}(1.137-2.622)$ \\
\hline Sales & $0.930(0.687-1.258)$ & $0.987(0.793-1.228)$ & $1.238(0.771-1.987)$ & $1.686^{c}(1.152-2.466)$ \\
\hline Agriculture & $0.805(0.503-1.291)$ & $1.194(0.867-1.646)$ & $1.378(0.785-2.421)$ & $1.801^{\mathrm{c}}(1.164-2.786)$ \\
\hline \multicolumn{5}{|l|}{ Household Wealth } \\
\hline Richest (ref) & 1 & 1 & 1 & 1 \\
\hline Poorest & $0.762(0.220-2.633)$ & $3.417^{c}(1.825-6.396)$ & $0.947(0.519-1.731)$ & $1.403(0.895-2.200)$ \\
\hline Poorer & $1.026(0.621-1.694)$ & $2.282^{c}(1.669-3.120)$ & $0.781(0.469-1.302)$ & $1.148(0.786-1.677)$ \\
\hline Middle & $1.241(0.899-1.714)$ & $1.979^{c}(1.583-2.475)$ & $0.775(0.508-1.183)$ & $0.997(0.723-1.375)$ \\
\hline Richer & $1.232^{\mathrm{a}}(0.977-1.553)$ & $1.704^{c}(1.448-2.006)$ & $0.928(0.652-1.32)$ & $0.968(0.734-1.277)$ \\
\hline \multicolumn{5}{|l|}{ Residence } \\
\hline Urban (ref) & 1 & 1 & 1 & 1 \\
\hline Rural & $1.092(0.864-1.381)$ & $0.927(0.785-1.093)$ & $0.748^{a}(0.556-1.006)$ & $0.779^{b}(0.624-0.974)$ \\
\hline \multicolumn{5}{|l|}{ Has health insurance } \\
\hline Yes (ref) & 1 & 1 & 1 & 1 \\
\hline No & $0.909(0.602-1.373)$ & $1.374^{\mathrm{b}}(1.011-1.867)$ & $0.320^{c}(0.246-0.417)$ & $0.829^{b}(0.699-0.983)$ \\
\hline \multicolumn{5}{|l|}{ Religion } \\
\hline Other Christian (ref) & 1 & 1 & 1 & 1 \\
\hline Catholic & $1.078(0.811-1.433)$ & $1.048(0.849-1.295)$ & $0.824(0.57-1.19)$ & $0.891(0.676-1.173)$ \\
\hline Traditionalist/ none & $1.324(0.254-6.898)$ & $2.043(0.625-6.678)$ & $1.128(0.75-1.695)$ & $1.137(0.840-1.538)$ \\
\hline Islam & $1.188(0.915-1.542)$ & $1.474^{c}(1.240-1.753)$ & $0.527^{b}(0.297-0.932)$ & $1.109(0.781-1.575)$ \\
\hline \multicolumn{5}{|c|}{ Need permission for medical help } \\
\hline Not a big problem (ref) & 1 & 1 & 1 & 1 \\
\hline Big problem & $1.247(0.771-2.016)$ & $1.130(0.792-1.611)$ & $1.036(0.64-1.678)$ & $1.015(0.705-1.462)$ \\
\hline \multicolumn{5}{|c|}{ Need money for medical help } \\
\hline Not a big problem (ref) & 1 & 1 & 1 & 1 \\
\hline Big problem & $1.041(0.835-1.297)$ & $0.812^{c}(0.697-0.947)$ & $1.297^{\mathrm{a}}(0.999-1.682)$ & $1.051(0.868-1.273)$ \\
\hline \multicolumn{5}{|l|}{$\begin{array}{l}\text { Distance to health } \\
\text { facility }\end{array}$} \\
\hline Not a big problem (ref) & 1 & 1 & 1 & 1 \\
\hline Big problem & $0.771^{a}(0.571-1.042)$ & $1.109(0.904-1.360)$ & $1.237(0.908-1.685)$ & $1.164(0.923-1.466)$ \\
\hline \multicolumn{5}{|c|}{ Do not want to visit health facility alone } \\
\hline Not a big problem (ref) & 1 & 1 & 1 & 1 \\
\hline Big problem & $1.728^{\mathrm{b}}(1.112-2.685)$ & $1.407^{b}(1.012-1.958)$ & $1.017(0.705-1.465)$ & $1.020(0.771-1.349)$ \\
\hline \multicolumn{5}{|c|}{ Heard family planning on radio last few months } \\
\hline Yes (ref) & 1 & 1 & 1 & 1 \\
\hline No & $0.885(0.648-1.208)$ & $0.800^{\mathrm{b}}(0.643-0.997)$ & $1.099(0.848-1.424)$ & $1.023(0.846-1.236)$ \\
\hline \multicolumn{5}{|c|}{ Heard family planning on TV last few months } \\
\hline Yes (ref) & 1 & 1 & 1 & 1 \\
\hline No & $0.790^{\mathrm{a}}(0.624-1.001)$ & $1.090(0.926-1.283)$ & $0.827(0.623-1.098)$ & $1.012(0.819-1.249)$ \\
\hline \multicolumn{5}{|c|}{ Heard family planning in print last few months } \\
\hline Yes (ref) & 1 & 1 & 1 & 1 \\
\hline No & $1.354^{\mathrm{b}}(1.066-1.720)$ & $1.105(0.934-1.308)$ & $0.552^{\mathrm{b}}(0.327-0.933)$ & $0.710(0.458-1.103)$ \\
\hline \multicolumn{5}{|l|}{ Region (Nigeria) } \\
\hline South West (ref) & 1 & 1 & - & - \\
\hline North Central & $0.462^{\complement}(0.320-0.666)$ & $0.962(0.765-1.209)$ & - & - \\
\hline
\end{tabular}


Table 3.2 continued:

\begin{tabular}{|c|c|c|c|c|}
\hline \multirow{4}{*}{$\begin{array}{l}\text { Background } \\
\text { characteristics }\end{array}$} & \multicolumn{2}{|l|}{ Nigeria } & \multicolumn{2}{|l|}{ Ghana } \\
\hline & Medium-access & Poor-access & Medium-access & Poor-access \\
\hline & \multicolumn{2}{|c|}{ Reference category: High access } & \multicolumn{2}{|c|}{ Reference category: High access } \\
\hline & Exp B $(95 \%$ Cl) & Exp B $(95 \% \mathrm{Cl})$ & Exp B $(95 \% \mathrm{Cl})$ & Exp B $(95 \% \mathrm{Cl})$ \\
\hline \multicolumn{5}{|l|}{ Region (Nigeria) } \\
\hline North East & $0.162^{c}(0.070-0.376)$ & $1.574^{b}(1.101-2.250)$ & - & - \\
\hline North West & $0.038^{c}(0.016-0.086)$ & $0.425^{\complement}(0.321-0.563)$ & - & - \\
\hline South East & $1.523^{b}(1.022-2.270)$ & $1.390^{\mathrm{b}}(1.01-01.913)$ & - & - \\
\hline South South & $1.100(0.780-1.550)$ & $0.965(0.755-1.233)$ & - & - \\
\hline \multicolumn{5}{|l|}{ Region } \\
\hline Greater Accra (ref) & - & - & 1 & 1 \\
\hline Western & - & - & $1.742^{\mathrm{b}}(1.097-2.765)$ & $0.868(0.610-1.234)$ \\
\hline Central & - & - & $0.754(0.463-1.229)$ & $0.691^{b}(0.494-0.966)$ \\
\hline Volta & - & - & $3.191^{\complement}(1.890-5.389)$ & $0.499^{c}(0.326-0.764)$ \\
\hline Eastern & - & - & $0.474^{c}(0.273-0.825)$ & $0.868(0.617-1.223)$ \\
\hline Ashanti & - & - & $1.912^{c}(1.264-2.893)$ & $1.103(0.808-1.505)$ \\
\hline Brong Ahafo & - & - & $1.826^{b}(1.123-2.969)$ & $0.658^{b}(0.451-0.960)$ \\
\hline Northern & - & - & $1.623(0.835-3.155)$ & $1.805^{b}(1.083-3.006)$ \\
\hline Upper East & - & - & $0.146^{c}(0.045-0.473)$ & 0.709 (0.424- 1.185) \\
\hline Upper West & - & - & $0.262^{b}(0.090-0.769)$ & $0.728(0.398-1.332)$ \\
\hline \multicolumn{5}{|l|}{ Ethnicity (Nigeria) } \\
\hline Yoruba (ref) & 1 & 1 & - & - \\
\hline Other minorities & $1.338^{\mathrm{a}}(0.961-1.864)$ & $1.953^{c}(1.566-2.436)$ & - & - \\
\hline Fulani & $1.961(0.436-8.812)$ & $3.352^{c}(1.699-6.612)$ & - & - \\
\hline Igbo & $2.134^{c}(1.471-3.096)$ & $1.922^{c}(1.448-2.551)$ & - & - \\
\hline Hausa & $4.820^{\circ}(2.139-10.861)$ & $11.842^{c}(7.766-18.059)$ & - & - \\
\hline \multicolumn{5}{|l|}{ Ethnicity (Ghana) } \\
\hline Akan (ref) & - & - & 1 & 1 \\
\hline Ga/Dangme & - & - & $1.294(0.775-2.159)$ & $1.142(0.790-1.650)$ \\
\hline Ewe & - & - & $1.044(0.687-1.584)$ & $1.059(0.780-1.437)$ \\
\hline Guan & - & - & $1.631(0.783-3.396)$ & $0.883(0.469-1.664)$ \\
\hline Mole-Dagbani & - & - & $1.184(0.754-1.860)$ & $1.061(0.754-1.493)$ \\
\hline Grusi & - & - & $0.942(0.490-1.811)$ & $0.682(0.422-1.103)$ \\
\hline Gurma & - & - & $0.948(0.523-1.720)$ & $0.883(0.563-1.385)$ \\
\hline Mande & - & - & $0.561(0.266-1.181)$ & $0.741(0.446-1.230)$ \\
\hline \multicolumn{5}{|c|}{ Attitude of the health workers } \\
\hline Not a big problem (ref) & 1 & 1 & - & - \\
\hline Big problem & $0.900(0.684-1.184)$ & $1.147(0.940-1.401)$ & - & - \\
\hline
\end{tabular}

${ }^{\mathrm{c}} p \leq 0.01 ;{ }^{\mathrm{b}} p \leq 0.05 ;{ }^{\mathrm{a}} p \leq 0.10$ (two-tailed test of significance) 
Table 3.3 shows the regression results on access to maternal health services in Nigeria and Ghana. In Nigeria's sample, women with primary or no education have higher odds to have poor-access $(\mathrm{OR}=1.387,95 \% \mathrm{Cl}: 1.140-1.687, p \leq 0.01)$ or low-access $(\mathrm{OR}=$ 1.786, 95\% Cl: 1.247- 2.557, $p \leq 0.01$ ) to maternal health services. In Nigeria's sample, women who are not working have higher odds to belong to the cluster of poor-access maternal health services only $(\mathrm{OR}=1.579,95 \% \mathrm{Cl} 1.081-2.307, p \leq 0.01)$. Compared to women in the white-collar occupational group, women in other occupational categories in Nigeria also have higher odds to belong to the poor-access cluster. Women in other occupational categories in Nigeria also have higher odds to belong to the poor-access cluster. Women in all household wealth quintiles have higher odds to have high- or poor-access to maternal health services; women without insurance have higher odds to have high or poor-access to maternal health services. Results for Ghana show that women with only primary $(\mathrm{OR}=1.38,95 \% \mathrm{Cl}: 1.036-1.838, p \leq 0.05)$ or no education $(\mathrm{OR}=1.542,95 \% \mathrm{Cl}$ : $1.115-2.132, p \leq 0.01)$ have higher odds of pooraccess to maternal health services. Only women in the agriculture occupational group have higher odds of high-access to maternal health services compared to women in the white-collar sector. Women without health insurance have higher odds of access to maternal health care services. 
Table 3.3: Odds ratio maternal health service: Nigeria and Ghana: Ref: Higher access (multinomial logistic regression)

\begin{tabular}{|c|c|c|c|}
\hline \multirow{4}{*}{ Background characteristics } & \multicolumn{3}{|l|}{ Nigeria } \\
\hline & High-access & Medium-access & Low-access \\
\hline & \multicolumn{3}{|c|}{ Reference category: Higher access } \\
\hline & Exp B $(95 \% \mathrm{Cl})$ & $\operatorname{Exp} B(95 \% \mathrm{Cl})$ & Exp B $(95 \% \mathrm{Cl})$ \\
\hline Maternal age & $0.977^{c}(0.963-0.992)$ & $0.980^{c}(0.967-0.994)$ & $0.956^{c}(0.930-0.982)$ \\
\hline Number of children alive & $1.101^{c}(1.045-1.16)$ & $1.087^{c}(1.033-1.145)$ & $1.113^{b}(1.016-1.220)$ \\
\hline \multicolumn{4}{|l|}{ Marital status } \\
\hline Married (ref) & 1 & 1 & 1 \\
\hline Never married & $1.081(0.722-1.619)$ & $0.894(0.600-1.332)$ & $1.550(0.676-3.551)$ \\
\hline Widowed/separated/ divorced & $1.252(0.871-1.800)$ & $1.055(0.729-1.527)$ & $1.248(0.634-2.459)$ \\
\hline \multicolumn{4}{|l|}{ Maternal Education } \\
\hline Secondary/ Higher (ref) & 1 & 1 & 1 \\
\hline No education & $1.145(0.902-1.454)$ & $0.690^{c}(0.524-0.909)$ & $1.849^{c}(1.247-2.742)$ \\
\hline Primary & $1.034(0.855-1.251)$ & $0.921(0.763-1.111)$ & $1.786^{c}(1.247-2.557)$ \\
\hline \multicolumn{4}{|l|}{ Maternal Occupation } \\
\hline White collar (ref) & 1 & 1 & 1 \\
\hline Not working & $1.052(0.779-1.421)$ & $1.097(0.847-1.422)$ & $1.754(0.752-4.093)$ \\
\hline Services and manual & $1.492^{c}(1.109-2.006)$ & $1.215(0.940-1.570)$ & $2.422^{b}(1.032-5.686)$ \\
\hline Sales & $1.289^{a}(0.979-1.698)$ & $1.300^{\mathrm{b}}(1.032-1.638)$ & $2.729^{b}(1.198-6.219)$ \\
\hline Agriculture & $1.697^{c}(1.187-2.428)$ & $1.298(0.922-1.826)$ & $4.253^{c}(1.741-10.392)$ \\
\hline \multicolumn{4}{|l|}{ Household Wealth } \\
\hline Richest (ref) & 1 & 1 & 1 \\
\hline Poorest & $4.726^{c}(2.982-7.489)$ & $1.531(0.855-2.740)$ & $3.230^{\circ}(1.668-6.255)$ \\
\hline Poorer & $2.750^{\circ}(2.018-3.748)$ & $1.104(0.796-1.533)$ & $1.580^{a}(0.921-2.711)$ \\
\hline Middle & $1.829^{c}(1.433-2.334)$ & $0.832(0.657-1.054)$ & $1.268(0.795-2.023)$ \\
\hline Richer & $1.476^{c}(1.221-1.785)$ & $0.733 c(0.616-0.873)$ & $0.665^{\mathrm{a}}(0.433-1.022)$ \\
\hline \multicolumn{4}{|l|}{ Residence } \\
\hline Urban (ref) & 1 & 1 & 1 \\
\hline Rural & $0.654^{c}(0.550-0.779)$ & $1.086(0.914-1.290)$ & $0.539^{c}(0.384-0.757)$ \\
\hline \multicolumn{4}{|l|}{ Has health insurance } \\
\hline Yes (ref) & 1 & 1 & 1 \\
\hline No & $1.167(0.770-1.770)$ & $0.789(0.574-1.085)$ & $0.563(0.274-1.156)$ \\
\hline \multicolumn{4}{|l|}{ Religion } \\
\hline Other Christian (ref) & 1 & 1 & 1 \\
\hline Catholic & $1.244^{\mathrm{a}}(0.970-1.595)$ & $1.582^{\mathrm{a}}(1.269-1.973)$ & $1.355(0.847-2.166)$ \\
\hline Traditionalist/ none & $0.643(0.249-1.661)$ & $0.470(0.165-1.340)$ & $0.379(0.034-4.263)$ \\
\hline Islam & $0.992(0.810-1.214)$ & $0.916(0.760-1.104)$ & $1.429^{a}(0.971-2.105)$ \\
\hline \multicolumn{4}{|l|}{ Need permission for medical help } \\
\hline Not a big problem (ref) & 1 & 1 & 1 \\
\hline Big problem & $0.990(0.709-1.384)$ & $0.781(0.540-1.128)$ & $0.620(0.312-1.232)$ \\
\hline \multicolumn{4}{|l|}{ Need money for medical help } \\
\hline Not a big problem (ref) & 1 & 1 & 1 \\
\hline Big problem & $0.996(0.843-1.178)$ & $0.879(0.744-1.037)$ & $1.033(0.768-1.389)$ \\
\hline \multicolumn{4}{|l|}{ Distance to health facility } \\
\hline Not a big problem (ref) & 1 & 1 & 1 \\
\hline Big problem & $1.211^{\mathrm{a}}(0.974-1.506)$ & $1.652^{\mathrm{c}}(1.327-2.057)$ & $1.109(0.760-1.620)$ \\
\hline \multicolumn{4}{|c|}{ Do not want to visit health facility alone } \\
\hline Not a big problem (ref) & 1 & 1 & 1 \\
\hline Big problem & $0.869(0.632-1.196)$ & $0.982(0.714-1.351)$ & $0.859(0.471-1.565)$ \\
\hline \multicolumn{4}{|c|}{ Heard family planning on radio last few months } \\
\hline Yes (ref) & 1 & 1 & 1 \\
\hline No & $1.008(0.797-1.275)$ & $0.776^{b}(0.612-0.983)$ & $0.418^{c}(0.240-0.730)$ \\
\hline
\end{tabular}




\begin{tabular}{|c|c|c|c|c|}
\hline \multirow[b]{2}{*}{ Poor-access } & \multicolumn{4}{|l|}{ Ghana } \\
\hline & High-access & Medium-access & Low-access & Poor-access \\
\hline & \multicolumn{4}{|c|}{ Reference category: Higher access } \\
\hline $\operatorname{Exp} B(95 \% \mathrm{Cl})$ & $\operatorname{Exp} B(95 \% \mathrm{Cl})$ & $\operatorname{Exp~B~}(95 \% \mathrm{Cl})$ & $\operatorname{Exp~B~}(95 \% \mathrm{Cl})$ & Exp B $(95 \% \mathrm{Cl})$ \\
\hline $0.965^{c}(0.950-0.980)$ & $0.977^{b}(0.957-0.997)$ & $0.984^{a}(0.963-1.005)$ & $1.020(0.995-1.047)$ & $0.961^{c}(0.940-0.983)$ \\
\hline $1.126^{c}(1.067-1.188)$ & $1.105^{\mathrm{b}}(1.016-1.201)$ & $1.088^{a}(0.998-1.185)$ & $1.000(0.899-1.113)$ & $1.239^{c}(1.134-1.353)$ \\
\hline 1 & 1 & 1 & 1 & 1 \\
\hline $1.382(0.922-2.072)$ & $0.981(0.699-1.377)$ & $0.713^{a}(0.495-1.026)$ & $0.970(0.628-1.498)$ & $0.880(0.604-1.281)$ \\
\hline $1.084(0.740-1.588)$ & $0.978(0.676-1.413)$ & $0.869(0.59-1.278)$ & $0.803(0.477-1.350)$ & $0.880(0.591-1.310)$ \\
\hline 1 & 1 & 1 & 1 & 1 \\
\hline $1.431^{c}(1.132-1.809)$ & $0.961(0.702-1.316)$ & $0.962(0.691-1.341)$ & $0.680^{a}(0.43-1.076)$ & $1.542^{c}(1.115-2.132)$ \\
\hline $1.387^{\circ}(1.140-1.687)$ & $0.975(0.742-1.280)$ & $1.202(0.914-1.580)$ & $0.594^{\mathrm{b}}(0.398-0.886)$ & $1.380^{\mathrm{b}}(1.036-1.838)$ \\
\hline 1 & 1 & 1 & 1 & 1 \\
\hline $1.579^{b}(1.081-2.307)$ & $1.074(0.644-1.79)$ & $0.771(0.474-1.254)$ & $0.889(0.535-1.476)$ & $1.076(0.500-2.313)$ \\
\hline $1.719^{c}(1.172-2.522)$ & 1.168 (0.699- 1.952) & $0.782(0.479-1.276)$ & $0.437^{c}(0.252-0.757)$ & $1.141(0.527-2.468)$ \\
\hline $2.001^{\complement}(1.395-2.871)$ & $0.824(0.511-1.329)$ & $0.576^{b}(0.368-0.903)$ & $0.628^{b}(0.4-0.987)$ & $0.779(0.371-1.636)$ \\
\hline $1.667^{\mathrm{b}}(1.076-2.584)$ & $1.781^{b}(1.022-3.104)$ & $0.735(0.426-1.266)$ & $0.539^{a}(0.277-1.05)$ & $1.226(0.561-2.682)$ \\
\hline 1 & 1 & 1 & 1 & 1 \\
\hline $6.592^{\mathrm{c}}(4.247-10.233)$ & $3.732^{\mathrm{c}}(2.171-6.415)$ & $3.826^{c}(2.16-6.775)$ & $1.889(0.863-4.135)$ & $20.631^{c}(10.086-42.199)$ \\
\hline $2.408^{c}(1.75-3.312)$ & $2.514^{c}(1.605-3.938)$ & $2.351^{\mathrm{c}}(1.479-3.737)$ & $1.679^{a}(0.949-2.97)$ & $10.228^{c}(5.352-19.544)$ \\
\hline $1.810^{\complement}(1.401-2.338)$ & $1.967^{c}(1.363-2.838)$ & $1.684^{c}(1.153-2.459)$ & $1.077(0.705-1.645)$ & $6.376^{c}(3.508-11.587)$ \\
\hline $1.237^{\mathrm{b}}(1.006-1.522)$ & $1.263(0.926-1.723)$ & $1.354^{a}(0.991-1.851)$ & $0.627^{\circ}(0.447-0.881)$ & $1.328(0.713-2.474)$ \\
\hline 1 & 1 & 1 & 1 & 1 \\
\hline $0.817^{b}(0.678-0.985)$ & 1.149 (0.889- 1.483) & $1.303^{a}(0.999-1.699)$ & $0.646^{\mathrm{b}}(0.453-0.921)$ & $2.139^{c}(1.600-2.86)$ \\
\hline 1 & 1 & 1 & 1 & 1 \\
\hline $1.570^{a}(0.926-2.661)$ & $1.146(0.923-1.422)$ & $1.260^{\mathrm{b}}(1.009-1.573)$ & 1.209 (0.919- 1.590) & $1.888^{\mathrm{c}}(1.500-2.376)$ \\
\hline 1 & 1 & 1 & 1 & 1 \\
\hline $0.952(0.709-1.278)$ & $0.710^{\mathrm{b}}(0.505-0.998)$ & $0.698^{\mathrm{b}}(0.492-0.99)$ & $0.496^{c}(0.300-0.820)$ & $0.679^{b}(0.470-0.980)$ \\
\hline 0.470 (0.154- 1.432) & $1.031(0.719-1.478)$ & $0.763(0.524-1.113)$ & $0.825(0.500-1.363)$ & $0.781(0.528-1.154)$ \\
\hline $0.976(0.783-1.215)$ & $1.112(0.644-1.921)$ & $1.294(0.724-2.314)$ & $2.472^{\mathrm{c}}(1.263-4.837)$ & $2.244^{\mathrm{c}}(1.351-3.726)$ \\
\hline 1 & 1 & 1 & 1 & 1 \\
\hline $1.016(0.730-1.415)$ & 1.097 (0.711- 1.691) & $0.816(0.525-1.267)$ & $0.478^{b}(0.23-0.993)$ & $0.840(0.532-1.328)$ \\
\hline 1 & 1 & 1 & 1 & 1 \\
\hline $1.304^{c}(1.100-1.547)$ & $0.999(0.794-1.256)$ & $1.239^{a}(0.977-1.572)$ & $0.668^{\mathrm{b}}(0.487-0.916)$ & 0.889 (0.693- 1.14) \\
\hline 1 & 1 & 1 & 1 & 1 \\
\hline $1.510^{c}(1.216-1.876)$ & $0.756^{a}(0.569-1.005)$ & 0.971 (0.729- 1.292) & $1.315(0.899-1.924)$ & 1.099 (0.827- 1.461) \\
\hline 1 & 1 & 1 & 1 & 1 \\
\hline $0.791(0.566-1.106)$ & $1.020(0.728-1.427)$ & $0.932(0.658-1.321)$ & $0.930(0.592-1.462)$ & $1.431^{b}(1.02-2.008)$ \\
\hline 1 & 1 & 1 & 1 & 1 \\
\hline $0.546^{c}(0.419-0.712)$ & $1.180(0.937-1.487)$ & $1.515^{c}(1.193-1.925)$ & $1.314^{a}(0.968-1.784)$ & $1.212(0.946-1.554)$ \\
\hline
\end{tabular}


Table 3.3 continued:

\begin{tabular}{|c|c|c|c|}
\hline \multirow{4}{*}{ Background characteristics } & \multicolumn{3}{|l|}{ Nigeria } \\
\hline & High-access & Medium-access & Low-access \\
\hline & \multicolumn{3}{|c|}{ Reference category: Higher access } \\
\hline & Exp B $(95 \% \mathrm{Cl})$ & $\operatorname{Exp~B~}(95 \% \mathrm{Cl})$ & $\operatorname{Exp~B~}(95 \% \mathrm{Cl})$ \\
\hline \multicolumn{4}{|c|}{ Heard family planning on TV last few months } \\
\hline Yes (ref) & 1 & 1 & 1 \\
\hline No & $0.917(0.768-1.095)$ & $0.934(0.785-1.112)$ & $0.928(0.670-1.286)$ \\
\hline \multicolumn{4}{|c|}{ Heard family planning in print last few months } \\
\hline Yes (ref) & 1 & 1 & 1 \\
\hline No & $0.861(0.708-1.047)$ & $0.877(0.729-1.053)$ & $1.384(0.885-2.165)$ \\
\hline \multicolumn{4}{|l|}{ Region (Nigeria) } \\
\hline South West (ref) & 1 & 1 & 1 \\
\hline North Central & $0.377^{c}(0.286-0.499)$ & $0.335^{c}(0.264-0.425)$ & $0.666(0.381-1.166)$ \\
\hline North East & $0.427^{c}(0.302-0.603)$ & $0.061^{c}(0.039-0.096)$ & $1.162(0.632-2.136)$ \\
\hline North West & $0.206^{c}(0.145-0.292)$ & $0.030^{c}(0.020-0.046)$ & $0.344^{c}(0.178-0.665)$ \\
\hline South East & $1.112(0.736-1.681)$ & 0.771 (0.554- 1.073) & $1.712(0.635-4.618)$ \\
\hline South South & $1.031(0.767-1.387)$ & $0.319^{c}(0.244-0.417)$ & $0.248^{a}(0.107-0.577)$ \\
\hline \multicolumn{4}{|l|}{ Region } \\
\hline Greater Accra (ref) & - & - & - \\
\hline Western & - & - & - \\
\hline Central & - & - & - \\
\hline Volta & - & - & - \\
\hline \multicolumn{4}{|l|}{ Region (continued) } \\
\hline Eastern & - & - & - \\
\hline Ashanti & - & - & - \\
\hline Brong Ahafo & - & - & - \\
\hline Northern & - & - & - \\
\hline Upper East & - & - & - \\
\hline Upper West & - & - & - \\
\hline \multicolumn{4}{|l|}{ Ethnicity (Nigeria) } \\
\hline Yoruba (ref) & 1 & 1 & 1 \\
\hline Other minorities & $0.541^{c}(0.412-0.71)$ & $0.987(0.780-1.249)$ & $1.223(0.677-2.208)$ \\
\hline Fulani & $0.681(0.405-1.143)$ & $0.868(0.400-1.883)$ & $0.897(0.376-2.139)$ \\
\hline Igbo & 0.759 (0.519- 1.109) & $1.808^{c}(1.336-2.447)$ & $1.014(0.378-2.722)$ \\
\hline Hausa & $0.862(0.59-1.259)$ & $1.177(0.760-1.822)$ & $2.231^{\mathrm{b}}(1.122-4.438)$ \\
\hline \multicolumn{4}{|l|}{ Ethnicity (Ghana) } \\
\hline Akan (ref) & - & - & - \\
\hline Ga/Dangme & - & - & - \\
\hline Ewe & - & - & - \\
\hline Guan & - & - & - \\
\hline Mole-Dagbani & - & - & - \\
\hline Grusi & - & - & - \\
\hline Gurma & - & - & - \\
\hline Mande & - & - & - \\
\hline \multicolumn{4}{|l|}{ Attitude of the health workers } \\
\hline Not a big problem (ref) & 1 & 1 & 1 \\
\hline Big problem & $1.707^{c}(1.314-2.217)$ & $1.361^{\mathrm{c}}(1.098-1.686)$ & $0.809(0.450-1.455)$ \\
\hline
\end{tabular}

${ }^{c} p \leq 0.01 ;{ }^{b} p \leq 0.05 ;{ }^{a} p \leq 0.10$ (two-tailed test of significance) 


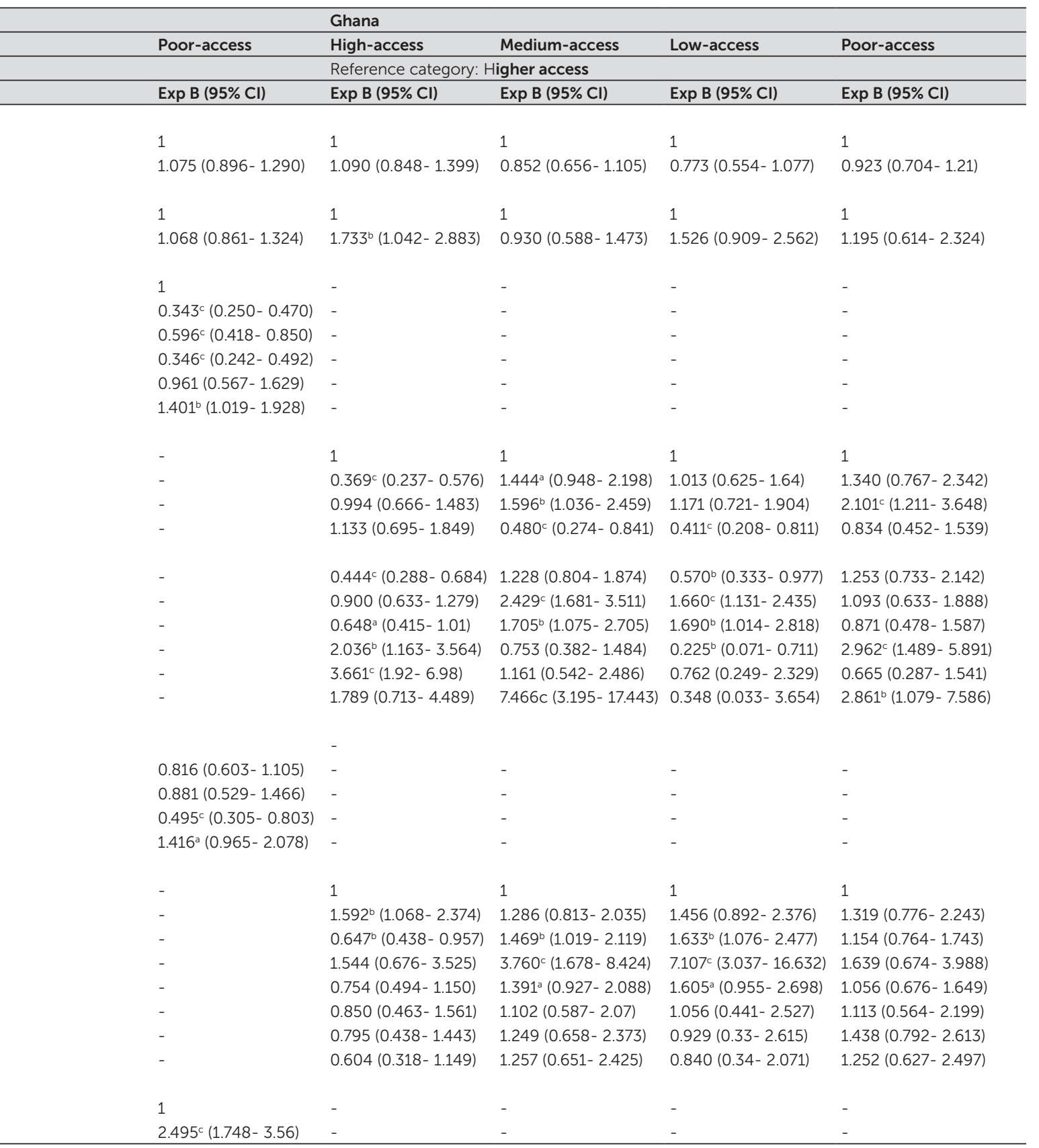




\subsection{DISCUSSION}

As shown by our results, access to reproductive health care services varies among women of reproductive age in Ghana and Nigeria. A large proportion of women in Ghana's and Nigeria's samples have poor access to family planning services. Most women do not have access to modern contraceptives. They use traditional birth control methods and do not have the means for needed services. These differences in access to maternal health services in both countries reflect a broader gap in health care use between women who access antenatal care at government hospitals for childbirth with a physician present and women who are not able to access such services. In particular, women in the low-access cluster are restricted to services at government health posts without skilled assistance during childbirth, or to services of antenatal care private vendors. This confirms that among women of reproductive age in Ghana and Nigeria, there is unequal access to reproductive health care services.

This suggests a dysfunctional organization structure that creates constraints to use preventive and medical procedures provided by well-trained professionals (Koblinsky et al., 1999; Odeyemi \& Nixon, 2013; Onah et al., 2006).

Our results show that educational attainment is associated with access to family planning and maternal health services. Low educational attainment reduces the ability to overcome access barriers, particularly to maternal health services. This finding supports similar results in other studies on the importance of education in improving access to reproductive health care services (Darroch \& Singh, 2013; Kabir et al., 2005; Mekonnen \& Mekonnen, 2002; Overbosch et al., 2004). Our results indicate that some women with low education intend to use contraceptives later while others use traditional contraceptive methods of family planning. Notably, the cluster with poor access to family planning services for a large part consists of women who have no intention of future contraceptive use. Lower-educated women seem to be less able to act on their intentions due to difficulties in overcoming access barriers or limited knowledge about the benefits of family planning (Ashford, 2003). The connection between education and socioeconomic status could also explain this observation because low education attainment, usually implies less access to resources (Ahmed, Creanga, Gillespie, \& Tsui, 2010; Darroch \& Singh, 2013; Ochako, Fotso, Ikamari, \& Khasakhala, 2011). The result further confirms what is known about the educational level as an indirect predictor of access to health care services (Solar \& Irwin, 2010).

The results suggest that wealth/finance related inequality in access to reproductive health care services is prominent in both Nigeria and Ghana. Considering finance- 
related inequality between the two countries, we find that women without insurance coverage in Ghana are less likely to access family planning services. This is dissimilar when compared to women without insurance in Nigeria; women in Nigeria who have poor-access to family planning opt for services such as traditional methods of contraception. These findings are consistent with other studies on the use of family planning services in the two countries and other parts of Africa (Darroch $\&$ Singh, 2013). This can be partially attributed to the inaccessibility of family planning services due to a cost-reducing scheme, which inadvertently increases preference for traditional contraceptives among some women (Singh et al., 2015). Another study also found a situation similar to Ghana among women in Burkina Faso and concluded that affordability of insurance premium varies by household income (Dong et al., 2005). The poor access to reproductive health care services in any of the wealth quintiles in Nigeria is expected considering the lack of insurance. The low coverage of insurance schemes such as the NHIS, particularly among informal workers or uneducated women, magnifies the effect of household wealth (Ibiwoye \& Adeleke, 2008; Odeyemi \& Nixon, 2013).

There is an association between maternal occupation and access to maternal health services in both countries. Other studies have also reported associations between care use and occupation (Addai, 2000; Mekonnen \& Mekonnen, 2002; Onah et al., 2006). However, where associations between maternal occupation and access to reproductive health care services are observable, disparity by type of livelihood is not unusual (Onah et al., 2006). We observe such differences between Ghana and Nigeria as well. In particular, the group of white-collar workers seems to have better access to family planning services in Ghana but no such differences are found for maternal care. The results for Nigeria are just the opposite; occupation does not explain poor access to family planning services in Nigeria but white-collar workers seem to have access to maternal care. In Nigeria, the cost of maternal health services has to be endured by women themselves while in Ghana, these services are available to women through the free maternal care policy $[28,29]$. Out-of-pocket payments for health have been consistently high in Nigeria compared to those in Ghana while insurance coverage is better in Ghana, particularly in the informal sector [27, 30].

\section{Study limitations}

This study has some limitations that need to be acknowledged. Cluster analysis method does not clarify the sources of variation among the observed variables. In that way, consequent information on the combinations of variables cannot be deduced for theoretical construct (Chow, 1998). There was not much variation in some response 
variables and they had to be excluded from the analysis. The inclusion of countryspecific variables helps to better reflect the women's situation but this also creates some dissimilarities in the country's analytical models.

\subsection{CONCLUSION}

This study provided evidence on inequalities in access to reproductive health care services within Ghana and Nigeria. A key observation is the varied composition of services available for use at different access levels. Several imperative factors contribute to inequality in access to these services. After controlling for the effects of maternalrelated variables, findings showed significant inequalities by educational attainment, household wealth, insurance status and woman's occupational type. Much of the inequality in access to family planning services that are seen in Nigeria and Ghana is related to education. The contribution of household wealth and insurance status in creating unequal access was also evidenced in the study. Health programs, which seek to stimulate the use of reproductive health care services in Ghana and Nigeria could take into account the variation in access reported in this study to assure the usercenteredness of these programs. It is important to identify and prioritize services for the needs of vulnerable groups. 
PATTERNS OF ACCESS TO REPRODUCTIVE HEALTH CARE SERVICES IN GHANA AND NIGERIA: RESULTS OF A CLUSTER ANALYSIS 



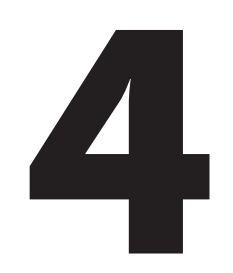

\section{EXAMINING TRENDS IN INEQUALITY IN THE USE OF REPRODUCTIVE HEALTH CARE SERVICES IN GHANA AND NIGERIA}

\section{Draws upon:}

Ogundele, O. J., Pavlova, M., \& Groot, W. (2018). Examining trends in inequality in the use of reproductive health care services in Ghana and Nigeria. BMC Pregnancy and Childbirth, 18(1), 492. 


\section{ABSTRACT}

\section{Background}

Equitable use of reproductive health care services is of critical importance since it may affect women's and children's health. Policies to reduce inequality in access to reproductive health care services are often general and frequently benefit the richer population. This is known as the inverse equity situation. We analyzed the magnitude and trends in wealth-related inequalities in the use of family planning, antenatal and delivery care services in Ghana and Nigeria. We also investigate horizontal inequalities in the determinants of reproductive health care service use over the years.

\section{Methods}

We use data from Ghana's (2003, 2008 and 2014) and Nigeria's (2003, 2008 and 2013) DHS. We use concentration curves and concentration indices to measure the magnitude of socioeconomic-related inequalities and horizontal inequality in the use of reproductive health care services.

\section{Results}

Exposure to family planning information via mass media, ANC at private facilities is higher among women in wealthier households. Health worker's assistance during pregnancy outside a facility, ANC at government facilities, and childbirth at home are more prevalent among women in poor households in both Ghana and Nigeria. C-section is unequally spread to the disadvantage of women in poorer households in Ghana and Nigeria. In Nigeria, women in wealthier households have considerably more unmet needs for family planning than in Ghana. Country inequality was persistent over time and women in poorer households in Nigeria experienced changes that are more inequitable over the years.

\section{Conclusion}

We observe horizontal inequalities among women who use reproductive health care. These inequalities did not reduce substantially over the years. The gains made in reducing inequality in the use of reproductive health care services are short-lived and erode over time, usually before the poorest population group can benefit. To reduce inequality in reproductive health care use, interventions should not only be pro-poor oriented, but they should also be sustainable and user-centered. 


\subsection{BACKGROUND}

Equitable provision of reproductive health care services is of critical importance since it affects, among others, individual and economic development and bears on universally recognized human rights. The loss of healthy life years due to morbidity or mortality resulting from reproductive ill-health among pregnant women is highest in SubSaharan Africa (Alkema et al., 2016). This increases poverty and impedes the economic growth of nations since it impacts on child development and women's labor force participation (AbouZahr \& Vaughan, 2000; Canning \& Schultz, 2012; Kabeer, 2012). In Sub-Saharan Africa countries, reproductive health care services are not affordable to everyone in need, leading to unequal access to care (Arsenault et al., 2013; Borghi et al., 2003; Honda, Randaoharison, \& Matsui, 2011).

As outlined in Chapter 1, policies to reduce inequality in access to reproductive health care services, particularly in Sub-Saharan African countries, often have unintended and unwanted consequences. Such as health providers' preference for urban and educated clients and the language barrier between provider and client (Mayhew, 2000). Likewise, due to the inefficient distribution of health resources, policies often fail the poorest population, inadvertently widening the poor-rich gap (Gilson et al., 2001). This is often referred to as the inverse equity hypothesis (Victora, Vaughan, Barros, Silva, \& Tomasi, 2000). Evidence on the relationship between government policies and access to services suggests that service delivery usually undermines benefits to the poor. For example, public health spending even though adequate can be allocated inefficiently and further precipitate between-group inequalities (Demery, 2000; Mills et al., 2012). Other studies have confirmed that access to health care innovations can be unequally distributed and to the advantage of richer households creating stratification in favor of the higher socioeconomic groups (Castro-Leal, Dayton, Demery, \& Mehra, 1999; Johnson et al., 2016; Makinen et al., 2000; Mutangadura et al., 2007; Okpani \& Abimbola, 2016; Zere et al., 2012; Zere et al., 2007). Though pre-sustainable development goals era illuminated maternal care, research showed that interventions that address family planning, as well as maternal health care, are inequitable (Barros et al., 2012).

In light of this, low- and middle-income countries have implemented pro-poor initiatives with the goal to advance equitable access to quality reproductive health care services. One example is the reproductive care services information channels in Nigeria (Abimbola et al., 2012). Another is the provision of insurance schemes and communitybased health programs in Ghana (Awoonor-Williams et al., 2013; Witter \& Garshong, 2009). Some of such successful interventions have been scaled-up, however with non- 
replicable successes (Nyonator et al., 2005; Okeke, Glick, \& Abubakar Isa, 2015). Both countries have put in place different health promotion schemes to attain a common goal of reducing the inequities associated with the delivery of reproductive health care services such as the fee exemption for maternity care in Ghana (Asante et al., 2007; NHIA) and the national health insurance scheme in Nigeria (Ibiwoye \& Adeleke, 2008). This study contributes to the literature by investigating the underlying mechanisms of inverse equity for subsequent initiatives for underserved populations (Victora et al., 2000). Ghana and Nigeria, through the sustainable development agenda, have agreed to foster equitable access to reproductive health care services (UNECA, 2015).

This Chapter analyzes the magnitude and trends in wealth-related inequality in the use of reproductive health care services (family planning and maternal care) in Ghana and Nigeria and provides insight into horizontal inequalities by describing the changes in the determinants of inequalities in the access to reproductive health care services over the years. An assessment of equity changes is essential to establish if policies addressing socioeconomic inequality improve the use of care. Nigeria and Ghana were selected based on the similarities and differences described previously in Chapter 1 of this dissertation.

As described in Chapter 1, in 2003, the fee exemption for maternity care commenced in four regions of Ghana (The Central, Northern, Upper West, and Upper East Regions), chosen because of the high poverty and maternal mortality levels and the low levels of supervised deliveries (Asante et al., 2007). This policy was expanded in 2005 to cover the other six regions of Ghana. Thus all pregnant women in Ghana are exempted from payments for maternity care services such as prenatal visits, childbirth care (physiological childbirth and childbirth with medical assistance), C-section, and postnatal visit in all facilities. Health insurance is compulsory for formal sector workers and voluntary for informal-sector workers and is reported to cover $65 \%$ of the population (Blanchet, Fink, \& Osei-Akoto, 2012; Odeyemi \& Nixon, 2013). The insurance premiums vary geographically and are ambiguously based on the ability to pay with no clear guideline to determine premium levels (Mensah, Oppong, \& Schmidt, 2010). However, significant differences in the use of maternity care persist (Asamoah, Agardh, Pettersson, \& Ostergren, 2014; Ayanore et al., 2016b; Dixon et al., 2013; Do, Soelaeman, \& Hotchkiss, 2015; Mensah et al., 2010). In a concurrent effort to promote access to health care, reproductive care services included, the communitybased health planning services in Ghana provide community-level services targeted at poor mothers and provide services including family planning, supervising delivery and maternity care (Nyonator et al., 2005). The community-based health planning 
services have been introduced to all districts/regions to facilitate access, especially for the population living further away from health care services.

Nigeria's national health insurance scheme, as described in Chapter 1, was initiated in 1999 and kicked off in 2005. It is a social health insurance scheme aimed at improving access to health care and reducing associated costs. It was piloted in six regions among civil servants and formal sector employees, targeting 5 percent of the population (Ibiwoye \& Adeleke, 2008). Coverage through the NHIS remains less than $5 \%$ of the Nigerian population (NHIS, 2011). To broaden coverage, the community-based health insurance scheme, flagged off in 2008, was made available to the general population and subsidized for households, particularly in rural communities. The scheme is organized by community members and covers family planning services, ANC, as well as vaginal childbirth (Onwujekwe et al., 2009). The community-based health insurance scheme allows for differences in premium rates, enrollment, and uptake of varied sexual and reproductive health care services across the country (Fakunle, Okunlola, Fajola, Ottih, \& Ilesanmi, 2014; Onwujekwe et al., 2009). Access to reproductive health care services in Nigeria remains underdeveloped and a large proportion of the population has no health coverage living most of the health expenditure to be borne by households.

The midwives service scheme was implemented in 2009 throughout the country as part of efforts to reach the rural communities and facilitate the adoption of skilled care among the population by improving the capacity of public primary health facilities (Fakunle et al., 2014). The allocation of midwives service scheme facilities is determined using geographic location as the factor with northeast and northwest regions emerging as a top priority, in part due to high maternal mortality rate and low access to services (Okeke et al., 2015). Though deemed to be making developments in implementation, highlighted setbacks include the non-availability of qualified midwives and retention of midwives (NPHCDA, 2018). Reports of horizontal variation in the use of reproductive health in the achievements of the midwives service scheme were reported (Okeke et al., 2015).

\subsection{METHODS}

\subsubsection{Data}

We used secondary data from the DHS. The surveys are conducted under an international program implemented by ICF International and funded by the USAID with contributions from UNICEF, UNFPA, WHO, and UNAIDS (DHS, 2018). Details of the 
survey methods used by the DHS are found in Chapter 1.

Analyses were performed using data from the women's response file, from the full DHS dataset of Ghana (2003, 2008, and 2014) and Nigeria (2003, 2008, and 2013). We use data from women who have had at least one birth in the 5 years prior to the survey.

\subsubsection{Measurement}

We consider family planning, ANC, and delivery care services as essential aspects of reproductive health care. The dependent variables to indicate exposure to or use of family planning, ANC, and delivery care services were grouped in similar themes based on the WHO recommendations (WHO, 2006). Box 4.1 shows the definition of the indicators used in the intervention areas examined. Responses to questions on the use of similar reproductive health care services were aggregated to produce one outcome variable (see Appendix C1 for grouping description). This was done to capture the different types of health care used during pregnancy. All dependent variables are dichotomized, taking the value 1 when a woman answered "Yes" to the questions and " 0 " if otherwise. The dependent variable indicating a woman's unmet need for family planning is coded as 1 when a woman answered "No" to the question if she wanted last birth and " 0 " if she answered "Yes". Control variables include a woman's age, marital status, occupation, location, and region of residence. The coding of the dependent and control variables used are indicated in Appendix C2.

Box 4.1: Definition of indicators by intervention area used for the equity analysis

\begin{tabular}{|c|c|}
\hline \multicolumn{2}{|l|}{ Indicators for family planning } \\
\hline Family planning info: Health facility & Percentage of women told of family planning at a health facility \\
\hline Family planning worker visit & Percentage of women who were visited by FP worker last 12 months \\
\hline Family planning: TV & $\begin{array}{l}\text { Percentage of women who heard family planning information on TV } \\
\text { last months }\end{array}$ \\
\hline Family planning: Print & $\begin{array}{l}\text { Percentage of women who got family planning information on a } \\
\text { newspaper last months }\end{array}$ \\
\hline Modern contraceptive & $\begin{array}{l}\text { Percentage of women who currently use by a modern method of } \\
\text { contraceptive }\end{array}$ \\
\hline $\begin{array}{l}\text { Information on pregnancy } \\
\text { complication }\end{array}$ & Percentage of women who were told about pregnancy complications \\
\hline Family planning: unmet need & $\begin{array}{l}\text { Percentage of women who wanted the last child later / wanted no } \\
\text { more }\end{array}$ \\
\hline \multicolumn{2}{|l|}{ Indicators for antenatal care } \\
\hline $\begin{array}{l}\text { Health workers assistance during } \\
\text { pregnancy outside a facility }\end{array}$ & Percentage of pregnant women who had care at an informal setting \\
\hline ANC: nurse assisted & $\begin{array}{l}\text { Percentage of pregnant women who got assistance from a nurse/ } \\
\text { midwife during pregnancy }\end{array}$ \\
\hline ANC: government health facility & $\begin{array}{l}\text { Percentage of pregnant women who received antenatal care at a form } \\
\text { of government/public health care center }\end{array}$ \\
\hline
\end{tabular}




\begin{tabular}{|c|c|}
\hline \multicolumn{2}{|l|}{ Indicators for antenatal care } \\
\hline ANC: Private health facility & $\begin{array}{l}\text { Percentage of pregnant women who received antenatal care at a form } \\
\text { the private health care center }\end{array}$ \\
\hline ANC: 1st trimester & $\begin{array}{l}\text { Percentage of pregnant women who received antenatal care in the } \\
\text { first } 12 \text { weeks of pregnancy }\end{array}$ \\
\hline ANC: $4+$ tetanus injection & $\begin{array}{l}\text { Percentage of pregnant women who received tetanus injections } \\
\text { before birth }\end{array}$ \\
\hline ANC: Home & Percentage of pregnant women who had antenatal care at a home \\
\hline \multicolumn{2}{|l|}{ Indicators for delivery care } \\
\hline Delivery: home & Percentage of pregnant women who had childbirth at a home \\
\hline Delivery: government health facility & $\begin{array}{l}\text { Percentage of pregnant women who had childbirth at a form of } \\
\text { government/public health care center }\end{array}$ \\
\hline Delivery: private health facility & $\begin{array}{l}\text { Percentage of pregnant women who had childbirth at a form of a } \\
\text { private health care center }\end{array}$ \\
\hline Birth assistance: Doctor & $\begin{array}{l}\text { Percentage of pregnant women who had childbirth assisted by a } \\
\text { Doctor }\end{array}$ \\
\hline C-section & Percentage of pregnant women who had C-section childbirth \\
\hline
\end{tabular}

\section{Household wealth}

To measure household wealth, asset ownership and living conditions available in each DHS dataset were used. Wealth was measured by ownership of some or all consumer items and residence characteristics including electricity, radio, television, refrigerator, bicycle, motorcycle, car or truck, non-mobile phone, water source, type of toilet facility, flooring, wall, and roofing materials. These were used to create a wealth index score by adopting Filmer and Pritchett (2001) principal component analysis approach to generate the indicator weights for the household assets and subsequently weighted scores for all assets that were summed to create a household wealth index. The types of assets owned were similar between Ghana and Nigeria, though local context implied differences in the consumer items and residence characteristics used in the index. The asset includes weights that varied between countries. This has been described as the local perception of wealth approach.

\subsubsection{Equity analysis}

To measure household wealth inequality in access to family planning, ANC, and delivery care services in Ghana and Nigeria over time, we use the concentration curve and associated index. The concentration curve plots the cumulative percentage of use of various health care services on the vertical axis ( $y$-axis) against the cumulative percentage of women ranked by their household wealth on the horizontal axis ( $x$-axis), beginning with the poorest and ending with the richest households. The equality line runs diagonally across the figure when women, irrespective of economic status have the same access to health care service, that is, all values on the $x$-axis equals all values 
on the $y$-axis (O'Donnell, Van Doorslaer, Wagstaff, \& Lindelow, 2008). A curve that lies below the equality line indicates that access to the health care service is concentrated among wealthier households. If the curve lies above the line of equality it implies the presence of inequity, that is, use of the health care service is concentrated among poorer households.

Concentration indices were used to assess the magnitudes and trend of horizontal inequity. Analyses were performed to measure absolute inequality in reproductive health care use. Concentration index range from -1.0 to +1.0; negative values of the concentration index indicate that the use of reproductive health care services is concentrated in poor households, positive values indicate among wealthy households, and 0 indicates the absence of household-wealth related inequality ( $O$ 'Donnell et al., 2008; Wagstaff, 1991). For computation, a more convenient formula for the concentration index (4.1) defines it in terms of the covariance between the health care outcome and the fractional rank in the household wealth distribution.

$$
\text { concentration index }=\frac{2}{u} \operatorname{cov}(h, r)
$$

where $h$ is the health care outcome of interest, $\mu$ is the mean of $h$ and $r$ is the fractional rank of an individual in the household wealth distribution. Additional analyses performed to test the null hypothesis of equality across groups to measure horizontal inequality, which is the hypothesis that the index is the same within a group. Comparison of the concentration indices within socioeconomic groups, including age, marital status, maternal occupation, location (rural or urban), and region of residence, was done using the homogeneity test as provided by O'Donnell, O'Neill, Van Ourti, and Walsh (2016). An alternative approach is the Oaxaca-Blinder decomposition. One of the limitations of this method is the possibility that the model might be sensitive to the omitted group in a particular indicator (Fortin, Lemieux, \& Firpo, 2011).

We used sampling weights for all statistical analyses. Data analyses were performed using STATA version 15.1.

\subsection{RESULTS}

Figures 4.1, 4.2 and 4.3 show the concentration curves of reproductive health care service use. At the end of the observed years, it appears that reproductive health care services are being used less by women in Nigeria compared with Ghana. The distribution 
of the outcome variables in the poorest 20 percent, richest 20 percent, and all women included in the analysis in Ghana and Nigeria (in Appendix C3) suggests changes in the proportion of women using reproductive services in both countries are irregular through the years. Additionally, the data suggest that the use of reproductive health care services did not increase substantially among women in the poorest households.

\subsubsection{Concentration curve of household wealth-related inequality in the use of reproductive health care services}

In Figure 4.1, the top image indicates that the curves of family planning information via print and TV lie distinctively furthest away from the line of equality over the observed years in Ghana, suggesting that these services are to the disadvantage of women in poor households. The bottom image of Figure 4.1 shows that all the curves describing the use of family planning services including the use of modern contraceptives lie below the line of equality observed in Nigeria. Lastly, the curve of unmet needs for family planning lies below the equality line and increased over the periods observed in Nigeria unlike in Ghana where the curve lies closely to the equality line all through (Appendix C4).

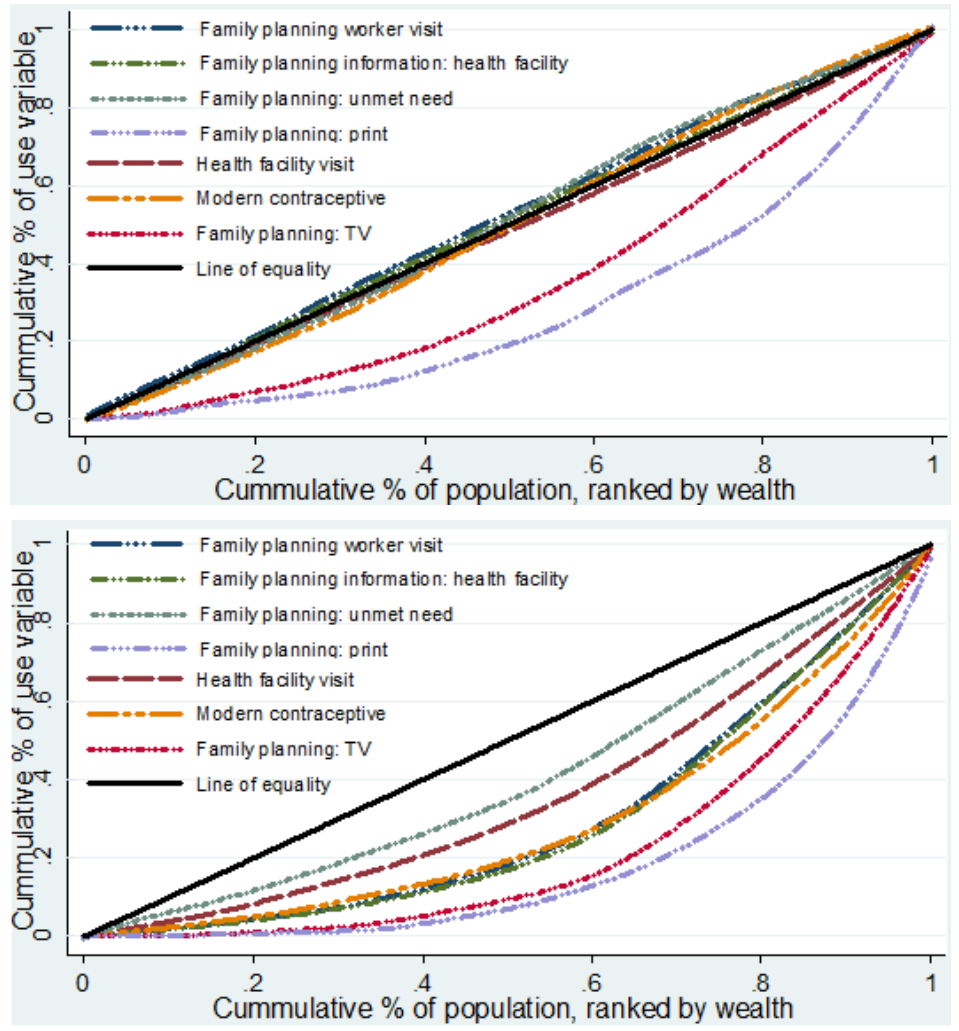

Figure 4.1: Concentration curves of use of family planning Ghana (2014) and Nigeria (2013) 
Figure 4.2 shows that in Ghana (top image), the concentration curve of ANC at private hospitals is below and furthest away from the equality line. Figure 4.2 (bottom image) suggests that all the examined ANC services are distributed to the disadvantage of women in poor households in Nigeria since the curve lies below the equity line. Other concentration curves depicting a woman's use of ANC lie close to the equality line (Appendix C5).
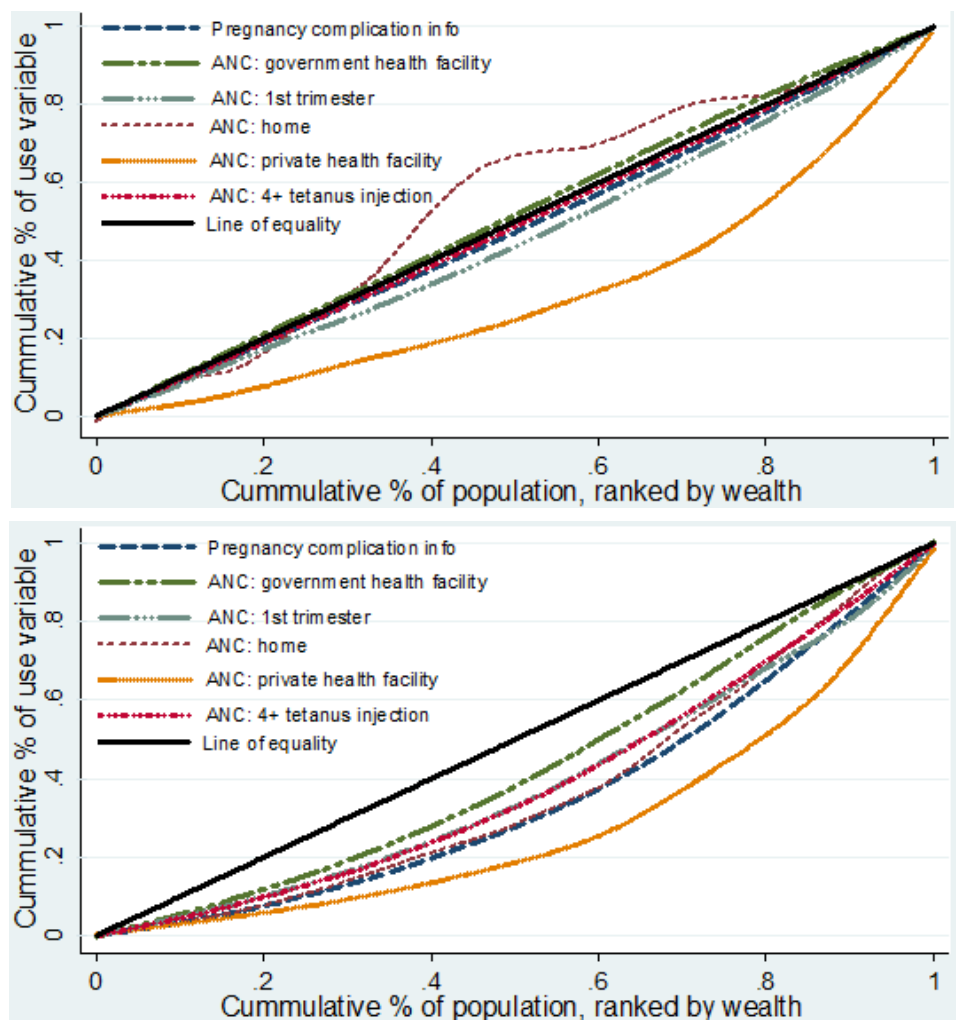

Figure 4.2: Concentration curves of use of antenatal care Ghana (2014) and Nigeria (2013)

Figures 4.3 (top and bottom images) show a clear picture of the curves for indicators associated with delivery care across the years in Ghana and Nigeria respectively. The curve of home births lies above the line of equality in both countries throughout the periods observed, indicating predominance among poor households (Appendix C6). Also, the curves depicting the use of private facility, C-section, and assistance by a doctor during child delivery appears to follow a similar pattern and lie furthest away below the equity line throughout the years observed (Appendix C6). 


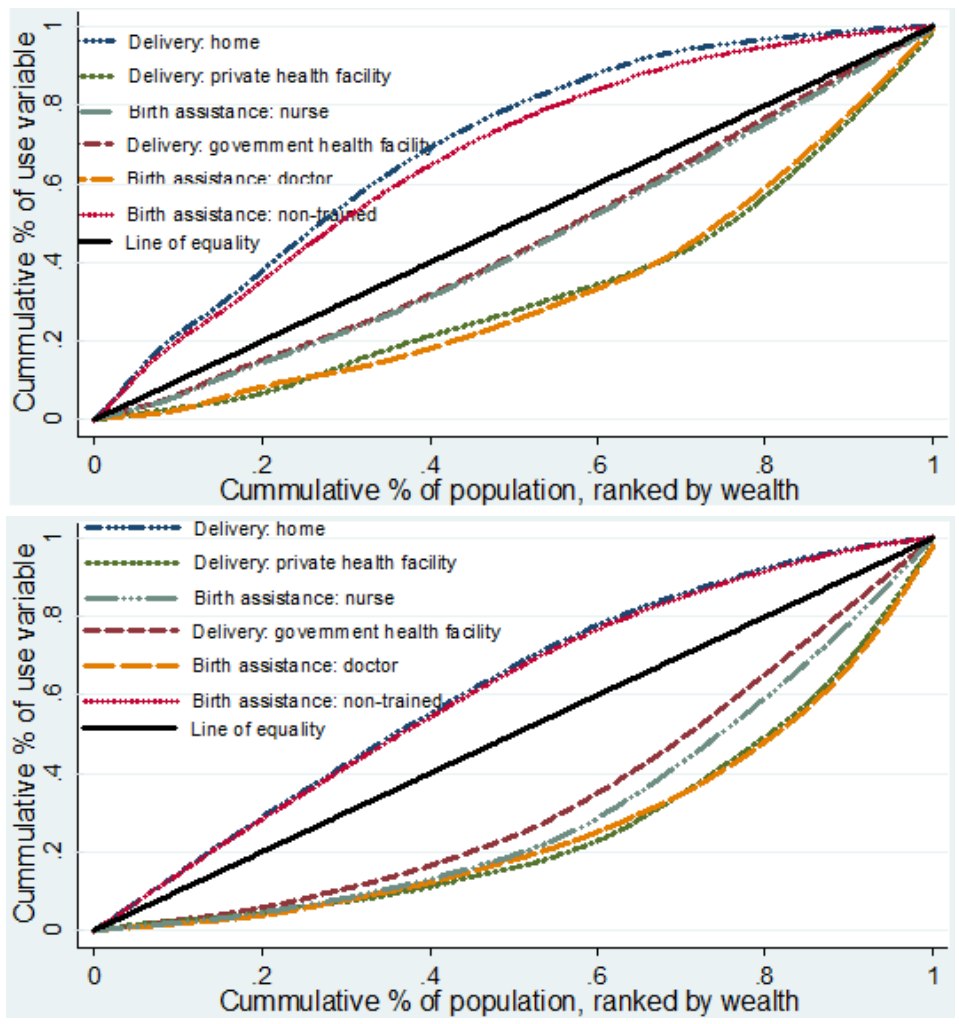

Figure 4.3: Concentration curves of use of Delivery care Ghana (2014) and Nigeria (2013)

\subsubsection{Concentration indices of household wealth-related inequality in the use of reproductive health care}

Table 4.1 shows that the value of the concentration index declined and remained positive for indicators of family planning information via TV $(+0.37$ to +0.28$)$, and print ( +0.54 to +0.42 ) from 2003 to 2014 in Ghana. Examination of these indicators by individual characteristics shows that concentration index values are positive and largest among women who are currently or were previously married, agrarians, or live in the Upper East region of Ghana. Concentration indices for indicators of the use of family planning information via TV or print medium are positive and high, above +0.43 , throughout the years observed in Nigeria, indicating concentration among wealthier households. Concentration index values for visits to health facilities or visits by family planning workers are negative between 2003 and 2014 in Ghana. Women who wanted to give birth no more or later and indicated unmet needs for family planning were insignificant across the years in Ghana. In Nigeria, concentration index values of unmet needs for family planning were positive and significant over the years. Individual 
estimates suggest that the degree of inequality associated with unmet needs for family planning is greatest among women in more wealthy households who are not working or in rural residence in Nigeria. Additional test results for group differences indicate that the magnitude of inequality of unmet needs for family planning is significantly different across occupation or residence types in the observed years.

Table 4.2 presents the values of the concentration indices of indicators of ANC services. Values show that the concentration index of health worker's assistance during pregnancy outside a facility declined slightly from -0.25 in 2003, to -0.21 in 2008 and peaked at -0.27 in 2014 in Ghana. Comparison across groups show that the use of health worker's assistance during pregnancy outside a facility was significantly different between occupation, place of residence, and region in Ghana for years 2003 and 2014; women in poor households, women in the professional / sales occupational category, living in an urban residence, or living in Greater Accra had the greatest negative concentration index values. In Nigeria, for health worker's assistance during pregnancy outside a facility, the values of concentration index indicate an increase from - 0.17 in 2003 to -0.21 in 2008 and 2013. Test results show significant differences between age and occupational groups, residence type as well as the region of residence; women who are 25-49 years, professional / sales occupation, in urban residences, or live in the South East region of Nigeria consistently have the greatest negative concentration index values. For the observed years, concentration index values of ANC in government hospitals increased from -0.02 to -0.04 in Ghana, from -0.04 to -0.07 in Nigeria. Urban-rural differences among women became insignificant after 2003 in Ghana and significant from 2008 in Nigeria. A similar increase in concentration index values is observed for ANC at private hospitals from +0.24 to +0.36 in Ghana and +0.20 to +0.27 in Nigeria. Over the observed years, the concentration index magnitude of home ANC indicator declined and remained negative in Ghana. However, a change in Nigeria from negative to positive was noted, $-0.23,-0.15$ and +0.05 . Results show that in the observed years, concentration index values increased for nurse assisted ANC in Nigeria, +0.33 to +0.39 , while it reduced in Ghana, +0.29 to +0.11 . 
Table 4.1: Concentration Indices with Covariates: Ghana (years 2000, 2005 \& 2014) and Nigeria (years 2003, 2008 \& 2013)

\begin{tabular}{|c|c|c|c|c|c|c|}
\hline \multirow[b]{2}{*}{ Service type / covariate } & \multicolumn{3}{|l|}{ Ghana } & \multicolumn{3}{|c|}{ Nigeria } \\
\hline & 2003 & 2008 & 2014 & 2003 & 2008 & 2013 \\
\hline Family planning info: health facility & -0.01 & -0.04 & $-0.04^{*}$ & $0.14^{*}$ & $0.14^{*}$ & $0.15^{*}$ \\
\hline \multicolumn{7}{|l|}{ Age group } \\
\hline $15-24$ & $0 *$ & 0.05 & -0.02 & 0.14 & $0.18^{*}$ & $0.18^{*}$ \\
\hline $25-49$ & -0.01 & -0.06 & $-0.05^{*}$ & $0.13^{*}$ & $0.12^{*}$ & $0.14^{*}$ \\
\hline \multicolumn{7}{|l|}{ Marital status } \\
\hline Never & -0.02 & -0.06 & -0.01 & 0.04 & 0.06 & 0.03 \\
\hline Currently / previously & -0.01 & -0.04 & $-0.04^{*}$ & $0.14 *$ & $0.14^{*}$ & $0.16^{*}$ \\
\hline \multicolumn{7}{|l|}{ Maternal occupation } \\
\hline Not working & 0.05 & 0 & 0 & 0.2 & $0.18^{*}$ & $0.21 *$ \\
\hline Professional / sales & 0 & -0.04 & $-0.07^{*}$ & $0.14^{*}$ & $0.09 *$ & $0.12^{*}$ \\
\hline Agriculture & -0.01 & -0.05 & 0 & 0.06 & $0.11^{*}$ & 0.08 \\
\hline Others & -0.02 & 0.02 & -0.01 & 0.13 & $0.16^{*}$ & $0.14^{*}$ \\
\hline \multicolumn{7}{|l|}{ Location } \\
\hline Urban & -0.02 & -0.01 & -0.02 & 0.02 & $0.08^{*}$ & $0.05^{*}$ \\
\hline Rural & -0.02 & -0.01 & -0.01 & 0.15 & $0.16^{*}$ & $0.19 *$ \\
\hline Family planning worker visit & $-0.09 *$ & -0.02 & -0.05 & $0.25^{*}$ & $0.39 *$ & $0.4^{*}$ \\
\hline \multicolumn{7}{|l|}{ Age group } \\
\hline $15-24$ & 0.01 & 0.05 & 0.01 & 0.22 & $0.49 *$ & $0.43^{*}$ \\
\hline $25-49$ & $-0.11^{*}$ & -0.04 & -0.06 & $0.25^{*}$ & $0.35^{*}$ & $0.38 *$ \\
\hline Marital status & $-0.09 *$ & -0.02 & -0.05 & $0.25^{*}$ & $0.39 *$ & $0.4^{*}$ \\
\hline Never & -0.1 & 0.09 & 0.03 & -0.03 & -0.04 & 0.01 \\
\hline Currently / previously & $-0.09 *$ & -0.02 & -0.06 & $0.26^{*}$ & $0.4^{*}$ & $0.41^{*}$ \\
\hline \multicolumn{7}{|l|}{ Maternal occupation } \\
\hline Not working & 0.07 & 0.15 & -0.03 & $0.43^{*}$ & $0.51^{*}$ & $0.44^{*}$ \\
\hline Professional / sales & $-0.18^{*}$ & -0.08 & 0.02 & $0.27^{*}$ & $0.32^{*}$ & $0.37^{*}$ \\
\hline Agriculture & -0.06 & $-0.14^{*}$ & 0.02 & 0.07 & $0.25^{*}$ & $0.2^{*}$ \\
\hline Others & -0.15 & 0.06 & -0.13 & 0.31 & $0.4^{*}$ & $0.36^{*}$ \\
\hline \multicolumn{7}{|l|}{ Location } \\
\hline Urban & $-0.15^{\star}$ & -0.01 & 0 & $0.18^{*}$ & $0.2^{*}$ & $0.14^{*}$ \\
\hline Rural & -0.04 & -0.02 & 0 & 0.22 & $0.39 *$ & $0.42^{*}$ \\
\hline Family planning: TV & $0.37^{*}$ & $0.4^{*}$ & $0.28 *$ & $0.5^{*}$ & $0.58^{*}$ & $0.56 *$ \\
\hline \multicolumn{7}{|l|}{ Age group } \\
\hline $15-24$ & $0.3^{*}$ & $0.37^{*}$ & $0.26 *$ & $0.44^{*}$ & $0.6^{*}$ & $0.61^{*}$ \\
\hline $25-49$ & $0.39 *$ & $0.41^{*}$ & $0.29 *$ & $0.51 *$ & $0.56^{*}$ & $0.53^{*}$ \\
\hline \multicolumn{7}{|l|}{ Marital status } \\
\hline Never & $0.2^{*}$ & $0.22^{*}$ & $0.2^{*}$ & 0.12 & $0.29 *$ & $0.31^{*}$ \\
\hline Currently / previously & $0.37^{*}$ & $0.42^{*}$ & $0.29 *$ & $0.51^{*}$ & $0.59^{*}$ & $0.57^{*}$ \\
\hline \multicolumn{7}{|l|}{ Maternal occupation } \\
\hline Not working & $0.33^{*}$ & $0.4^{*}$ & $0.24 *$ & $0.52^{*}$ & $0.64 *$ & $0.65^{*}$ \\
\hline Professional / sales & $0.23^{*}$ & $0.27^{*}$ & $0.15^{*}$ & $0.45^{*}$ & $0.48^{*}$ & $0.5^{*}$ \\
\hline Agriculture & $0.32 *$ & $0.46^{*}$ & $0.32 *$ & $0.51^{*}$ & $0.41^{*}$ & $0.32^{*}$ \\
\hline Others & $0.27^{*}$ & $0.26^{*}$ & $0.21 *$ & $0.42^{*}$ & $0.51^{*}$ & $0.49 *$ \\
\hline \multicolumn{7}{|l|}{ Location } \\
\hline Urban & $0.17^{*}$ & $0.19 *$ & $0.13^{*}$ & $0.29 *$ & $0.28 *$ & $0.25^{*}$ \\
\hline Rural & $0.32^{*}$ & $0.44^{*}$ & $0.35^{*}$ & $0.39 *$ & $0.63^{*}$ & $0.63^{*}$ \\
\hline Family planning: print & $0.54^{*}$ & $0.52^{*}$ & $0.42^{*}$ & $0.43^{*}$ & $0.65^{*}$ & $0.64 *$ \\
\hline \multicolumn{7}{|l|}{ Age group } \\
\hline $15-24$ & $0.5^{*}$ & $0.56^{*}$ & 0.25 & $0.4^{*}$ & $0.63^{*}$ & $0.62 *$ \\
\hline $25-49$ & $0.55^{*}$ & $0.51^{*}$ & $0.44^{*}$ & $0.44^{*}$ & $0.64^{*}$ & $0.62 *$ \\
\hline
\end{tabular}


Table 4.1 continued:

\begin{tabular}{|c|c|c|c|c|c|c|}
\hline \multirow[b]{2}{*}{ Service type / covariate } & \multicolumn{3}{|l|}{ Ghana } & \multicolumn{3}{|c|}{ Nigeria } \\
\hline & 2003 & 2008 & 2014 & 2003 & 2008 & 2013 \\
\hline \multicolumn{7}{|l|}{ Marital status } \\
\hline Never & 0.28 & 0.5 & 0.36 & 0.22 & $0.36^{*}$ & 0.26 \\
\hline Currently / previously & $0.55^{*}$ & $0.52^{*}$ & $0.43^{*}$ & $0.45^{*}$ & $0.66^{*}$ & $0.65^{*}$ \\
\hline Not working & $0.45^{*}$ & $0.46 *$ & 0.14 & $0.42^{*}$ & $0.7^{*}$ & $0.73^{*}$ \\
\hline Professional / sales & $0.4^{*}$ & $0.43^{*}$ & $0.31^{*}$ & $0.4^{*}$ & $0.57^{*}$ & $0.56^{*}$ \\
\hline Agriculture & $0.43^{*}$ & $0.55^{*}$ & 0.32 & 0.47 & $0.42 *$ & $0.5^{*}$ \\
\hline Others & $0.43^{*}$ & $0.37^{*}$ & 0.19 & $0.38^{*}$ & $0.57^{*}$ & $0.59 *$ \\
\hline \multicolumn{7}{|l|}{ Location } \\
\hline Urban & $0.31 *$ & $0.36 *$ & $0.26 *$ & $0.34 *$ & $0.39 *$ & $0.36^{*}$ \\
\hline Rural & $0.45^{*}$ & $0.54 *$ & $0.45^{*}$ & $0.3^{*}$ & $0.67^{*}$ & $0.67^{*}$ \\
\hline Modern contraceptive & 0.01 & -0.05 & $-0.06^{*}$ & 0.03 & $-0.04^{*}$ & -0.02 \\
\hline \multicolumn{7}{|l|}{ Age group } \\
\hline $15-24$ & 0.01 & -0.05 & $-0.08 *$ & 0.06 & 0.02 & 0.02 \\
\hline $25-49$ & 0.01 & -0.04 & $-0.05^{*}$ & 0.03 & $-0.05^{*}$ & -0.02 \\
\hline \multicolumn{7}{|l|}{ Marital status } \\
\hline Never & 0.04 & -0.17 & -0.06 & 0.07 & 0.05 & $0.07^{*}$ \\
\hline Currently / previously & 0.01 & -0.04 & $-0.06^{*}$ & 0.03 & $-0.04^{*}$ & -0.02 \\
\hline \multicolumn{7}{|l|}{ Maternal occupation } \\
\hline Not working & -0.01 & -0.08 & -0.05 & 0.05 & -0.05 & -0.03 \\
\hline Professional / sales & 0.02 & -0.04 & $-0.08 *$ & 0.02 & -0.03 & -0.01 \\
\hline Agriculture & 0.01 & $-0.07^{*}$ & 0 & 0.04 & $-0.09 *$ & -0.03 \\
\hline Others & -0.02 & -0.01 & $-0.07^{*}$ & -0.02 & 0 & -0.05 \\
\hline \multicolumn{7}{|l|}{ Location } \\
\hline Urban & -0.02 & -0.06 & $-0.07^{*}$ & 0.02 & -0.02 & 0 \\
\hline Rural & 0.02 & -0.05 & -0.02 & 0.03 & $-0.04^{*}$ & -0.03 \\
\hline $\begin{array}{l}\text { Information on pregnancy } \\
\text { complication }\end{array}$ & $0.08 *$ & $0.07^{*}$ & $0.02 *$ & $0.13^{*}$ & $0.12 *$ & $0.1^{*}$ \\
\hline \multicolumn{7}{|l|}{ Age group } \\
\hline $15-24$ & $0.06^{*}$ & $0.06 *$ & 0 & $0.16^{*}$ & $0.12^{*}$ & $0.1^{*}$ \\
\hline $25-49$ & $0.09 *$ & $0.07^{*}$ & $0.02^{*}$ & $0.11 *$ & $0.11^{*}$ & $0.09 *$ \\
\hline \multicolumn{7}{|l|}{ Marital status } \\
\hline Never & $0.15^{*}$ & 0.1 & 0 & 0.1 & 0.1 & $0.08^{*}$ \\
\hline Currently / previously & $0.08^{*}$ & $0.07^{*}$ & $0.02 *$ & $0.13^{*}$ & $0.12^{*}$ & $0.1^{*}$ \\
\hline \multicolumn{7}{|l|}{ Maternal occupation } \\
\hline Not working & $0.15^{*}$ & 0 & 0 & $0.17^{*}$ & $0.15^{*}$ & $0.1^{*}$ \\
\hline Professional / sales & $0.06^{*}$ & $0.04^{*}$ & 0 & $0.11 *$ & $0.09 *$ & $0.09 *$ \\
\hline Agriculture & 0.1 & $0.06^{*}$ & 0 & 0.1 & 0 & $0.07^{*}$ \\
\hline Others & 0.1 & 0 & $0.03^{*}$ & $0.11 *$ & $0.11 *$ & $0.08^{*}$ \\
\hline \multicolumn{7}{|l|}{ Location } \\
\hline Urban & 0 & 0 & $0.02 *$ & $0.08^{*}$ & $0.07^{*}$ & $0.05^{*}$ \\
\hline Rural & $0.07^{*}$ & $0.07^{*}$ & 0 & $0.09 *$ & $0.1^{*}$ & $0.08^{*}$ \\
\hline Family planning: unmet need & 0.01 & 0.01 & -0.02 & 0.05 & $0.18^{*}$ & $0.18^{*}$ \\
\hline \multicolumn{7}{|l|}{ Age group } \\
\hline $15-24$ & $0.08^{*}$ & 0.06 & 0.05 & 0.11 & $0.25^{*}$ & $0.22^{*}$ \\
\hline $25-49$ & -0.02 & -0.01 & -0.03 & 0.03 & $0.17^{*}$ & $0.17^{*}$ \\
\hline \multicolumn{7}{|l|}{ Marital status } \\
\hline Never & -0.03 & $0.09 *$ & -0.03 & 0.03 & 0.04 & 0 \\
\hline Currently / previously & 0.01 & -0.01 & -0.02 & 0.05 & $0.19 *$ & $0.19 *$ \\
\hline
\end{tabular}


Table 4.1 continued:

\begin{tabular}{|c|c|c|c|c|c|c|}
\hline \multirow[b]{2}{*}{ Service type / covariate } & \multicolumn{3}{|c|}{ Ghana } & \multicolumn{3}{|c|}{ Nigeria } \\
\hline & 2003 & 2008 & 2014 & 2003 & 2008 & 2013 \\
\hline \multicolumn{7}{|l|}{ Maternal occupation } \\
\hline Not working & 0.06 & 0.03 & -0.04 & 0.08 & $0.21 *$ & $0.29 *$ \\
\hline Professional / sales & -0.04 & $-0.09 *$ & $-0.11 *$ & 0.07 & $0.16^{*}$ & $0.21 *$ \\
\hline Agriculture & $0.07^{*}$ & $0.09 *$ & 0.08 & 0.06 & $0.12^{*}$ & 0.01 \\
\hline Others & -0.02 & -0.06 & -0.07 & 0.11 & $0.3^{*}$ & $0.19 *$ \\
\hline \multicolumn{7}{|l|}{ Location } \\
\hline Urban & -0.02 & -0.08 & $-0.11 *$ & 0.07 & 0.04 & 0.02 \\
\hline Rural & $0.07^{*}$ & $0.07 *$ & 0.06 & 0.03 & $0.22 *$ & $0.21 *$ \\
\hline
\end{tabular}

Note: Magnitudes of regional variation is available in Appendix C7

Table 4.2: Concentration Indices with Covariates: Ghana (years 2000, 2005 \& 2014) and Nigeria (years 2003, 2008 \& 2013)

\begin{tabular}{|c|c|c|c|c|c|c|}
\hline \multirow[b]{2}{*}{ Service type / covariate } & \multicolumn{3}{|l|}{ Ghana } & \multicolumn{3}{|l|}{ Nigeria } \\
\hline & 2003 & 2008 & 2014 & 2003 & 2008 & 2013 \\
\hline $\begin{array}{l}\text { Health worker's assistance during } \\
\text { pregnancy outside a facility }\end{array}$ & $-0.25^{*}$ & $-0.21 *$ & $-0.27 *$ & $-0.17^{*}$ & $-0.21^{*}$ & $-0.21 *$ \\
\hline \multicolumn{7}{|l|}{ Age group } \\
\hline $15-24$ & $-0.23^{*}$ & -0.06 & $-0.19 *$ & $-0.13^{*}$ & $-0.14^{*}$ & $-0.15^{*}$ \\
\hline $25-49$ & $-0.26 *$ & $-0.25^{*}$ & $-0.29 *$ & $-0.19 *$ & $-0.23^{*}$ & $-0.23 *$ \\
\hline \multicolumn{7}{|l|}{ Marital status } \\
\hline Never & $-0.21^{*}$ & -0.2 & -0.2 & -0.23 & $-0.15^{*}$ & $-0.17^{*}$ \\
\hline Currently / previously & $-0.25^{*}$ & $-0.21 *$ & $-0.28 *$ & $-0.17^{*}$ & $-0.21 *$ & $-0.21 *$ \\
\hline \multicolumn{7}{|l|}{ Maternal occupation } \\
\hline Not working & $-0.29 *$ & $-0.32^{*}$ & $-0.2^{*}$ & $-0.16^{*}$ & $-0.17^{*}$ & $-0.17^{*}$ \\
\hline Professional / sales & $-0.35^{*}$ & $-0.2^{*}$ & $-0.29 *$ & $-0.19 *$ & $-0.28 *$ & $-0.26 *$ \\
\hline Agriculture & $-0.07^{*}$ & -0.03 & -0.08 & $-0.14^{*}$ & $-0.09 *$ & $-0.1 *$ \\
\hline Others & $-0.29 *$ & $-0.24^{*}$ & $-0.23 *$ & $-0.18^{*}$ & $-0.23 *$ & $-0.23 *$ \\
\hline \multicolumn{7}{|l|}{ Location } \\
\hline Urban & $-0.3^{*}$ & $-0.18^{*}$ & $-0.19 *$ & $-0.22^{*}$ & $-0.27^{*}$ & $-0.24 *$ \\
\hline Rural & $-0.09 *$ & $-0.07^{*}$ & $-0.17^{*}$ & $-0.1^{\star}$ & $-0.13^{*}$ & $-0.11^{*}$ \\
\hline ANC: nurse assisted & $0.29 *$ & $0.24 *$ & $0.11^{*}$ & $0.33^{*}$ & $0.4^{*}$ & $0.39 *$ \\
\hline \multicolumn{7}{|l|}{ Age group } \\
\hline $15-24$ & $0.26^{*}$ & $0.19 *$ & $0.11 *$ & $0.32^{*}$ & $0.4^{*}$ & $0.39 *$ \\
\hline $25-49$ & $0.3^{*}$ & $0.25^{*}$ & $0.11^{*}$ & $0.33^{*}$ & $0.39 *$ & $0.39 *$ \\
\hline \multicolumn{7}{|l|}{ Marital status } \\
\hline Never & 0.1 & $0.11 *$ & $0.09 *$ & 0.15 & $0.23^{*}$ & $0.16 *$ \\
\hline Currently / previously & $0.29 *$ & $0.24 *$ & $0.11 *$ & $0.34^{*}$ & $0.4^{*}$ & $0.4^{*}$ \\
\hline \multicolumn{7}{|l|}{ Maternal occupation } \\
\hline Not working & $0.26 *$ & $0.2^{*}$ & $0.08^{*}$ & $0.46^{*}$ & $0.49 *$ & $0.47^{*}$ \\
\hline Professional / sales & $0.16 *$ & $0.14^{*}$ & $0.04^{*}$ & $0.3 *$ & $0.35^{*}$ & $0.38^{*}$ \\
\hline Agriculture & $0.21 *$ & $0.26 *$ & $0.12^{*}$ & $0.28 *$ & $0.26^{*}$ & $0.2^{*}$ \\
\hline Others & $0.21 *$ & $0.14^{*}$ & $0.08^{*}$ & $0.29 *$ & $0.4^{*}$ & $0.38^{*}$ \\
\hline \multicolumn{7}{|l|}{ Location } \\
\hline Urban & $0.06 *$ & $0.06^{*}$ & 0.02 & $0.16^{*}$ & $0.17^{*}$ & $0.12^{*}$ \\
\hline Rural & $0.24^{*}$ & $0.24^{*}$ & $0.12^{*}$ & $0.31^{*}$ & $0.41^{*}$ & $0.43^{*}$ \\
\hline
\end{tabular}


Table 4.2 continued:

\begin{tabular}{|c|c|c|c|c|c|c|}
\hline \multirow[b]{2}{*}{ Service type / covariate } & \multicolumn{3}{|l|}{ Ghana } & \multicolumn{3}{|l|}{ Nigeria } \\
\hline & 2003 & 2008 & 2014 & 2003 & 2008 & 2013 \\
\hline ANC: government health facility & $-0.02 *$ & $-0.03^{*}$ & $-0.04 *$ & -0.04 & $-0.07^{*}$ & $-0.07^{*}$ \\
\hline \multicolumn{7}{|l|}{ Age group } \\
\hline $15-24$ & -0.01 & -0.01 & -0.02 & -0.04 & $-0.05^{*}$ & $-0.06 *$ \\
\hline $25-49$ & $-0.02 *$ & $-0.04 *$ & $-0.05^{*}$ & -0.04 & $-0.07^{*}$ & $-0.07^{*}$ \\
\hline \multicolumn{7}{|l|}{ Marital status } \\
\hline Never & $-0.08 *$ & 0.02 & 0 & -0.15 & -0.03 & -0.05 \\
\hline Currently / previously & $-0.02^{*}$ & $-0.03^{*}$ & $-0.04 *$ & -0.04 & $-0.07^{*}$ & $-0.07^{*}$ \\
\hline \multicolumn{7}{|l|}{ Maternal occupation } \\
\hline Not working & -0.01 & $-0.04 *$ & $-0.03 *$ & -0.02 & $-0.08^{*}$ & $-0.08 *$ \\
\hline Professional / sales & -0.02 & -0.02 & $-0.05^{*}$ & $-0.05^{*}$ & $-0.08^{*}$ & $-0.08^{*}$ \\
\hline Agriculture & 0 & -0.01 & 0 & -0.06 & 0 & -0.01 \\
\hline Others & -0.03 & $-0.05^{*}$ & -0.02 & -0.07 & $-0.1 *$ & $-0.09 *$ \\
\hline \multicolumn{7}{|l|}{ Location } \\
\hline Urban & $-0.03^{*}$ & -0.01 & $-0.04^{*}$ & -0.03 & $-0.1 *$ & $-0.07 *$ \\
\hline Rural & 0 & $-0.02 *$ & $-0.02 *$ & -0.03 & $-0.03^{*}$ & $-0.03 *$ \\
\hline ANC: private health facility & $0.24^{*}$ & $0.3^{*}$ & $0.36^{*}$ & $0.2^{*}$ & $0.23 *$ & $0.27^{*}$ \\
\hline \multicolumn{7}{|l|}{ Age group } \\
\hline $15-24$ & 0.15 & 0.05 & 0.21 & $0.23^{*}$ & $0.21 *$ & $0.27^{*}$ \\
\hline $25-49$ & $0.27^{*}$ & $0.34 *$ & $0.38 *$ & $0.19 *$ & $0.23 *$ & $0.25^{*}$ \\
\hline \multicolumn{7}{|l|}{ Marital status } \\
\hline Never & $0.44^{*}$ & -0.1 & 0.26 & $0.37^{*}$ & 0.17 & $0.17^{*}$ \\
\hline Currently / previously & $0.23^{*}$ & $0.33^{*}$ & $0.36^{*}$ & $0.19 *$ & $0.23^{*}$ & $0.27^{*}$ \\
\hline \multicolumn{7}{|l|}{ Maternal occupation } \\
\hline Not working & 0.12 & $0.42^{*}$ & $0.27^{*}$ & $0.24^{*}$ & $0.35^{*}$ & $0.37^{*}$ \\
\hline Professional / sales & $0.17^{*}$ & 0.16 & $0.32^{*}$ & $0.18^{*}$ & $0.23 *$ & $0.27^{*}$ \\
\hline Agriculture & 0.15 & 0.12 & -0.08 & 0.2 & 0.01 & 0.01 \\
\hline Others & $0.19 *$ & $0.32^{*}$ & $0.27^{*}$ & $0.24^{*}$ & $0.26 *$ & $0.33^{*}$ \\
\hline \multicolumn{7}{|l|}{ Location } \\
\hline Urban & $0.17^{*}$ & 0.11 & $0.26^{*}$ & $0.14^{*}$ & $0.21 *$ & $0.17^{*}$ \\
\hline Rural & 0.09 & $0.32 *$ & $0.26 *$ & 0.18 & $0.15^{*}$ & $0.21^{*}$ \\
\hline ANC: 1st trimester & $0.09 *$ & $0.09 *$ & $0.07^{*}$ & -0.01 & 0.03 & 0.04 \\
\hline \multicolumn{7}{|l|}{ Age group } \\
\hline $15-24$ & $0.08 *$ & 0.08 & 0.05 & 0.05 & -0.03 & 0.01 \\
\hline $25-49$ & $0.09 *$ & $0.09 *$ & $0.07^{*}$ & -0.03 & $0.04 *$ & 0.04 \\
\hline \multicolumn{7}{|l|}{ Marital status } \\
\hline Never & 0.03 & 0.01 & $0.08^{*}$ & 0.24 & -0.06 & 0.02 \\
\hline Currently / previously & $0.09 *$ & $0.1^{*}$ & $0.07^{*}$ & -0.02 & 0.03 & 0.04 \\
\hline \multicolumn{7}{|l|}{ Maternal occupation } \\
\hline Not working & $0.13^{*}$ & $0.1^{*}$ & $0.08^{*}$ & 0.07 & 0.02 & 0.06 \\
\hline Professional / sales & $0.07^{*}$ & $0.07^{*}$ & $0.05^{*}$ & -0.05 & $0.06 *$ & 0.04 \\
\hline Agriculture & $0.06^{*}$ & 0.06 & 0.02 & 0 & 0.01 & -0.06 \\
\hline Others & 0.04 & 0.04 & $0.05^{*}$ & -0.03 & $0.1^{*}$ & $0.11^{*}$ \\
\hline \multicolumn{7}{|l|}{ Location } \\
\hline Urban & $0.06 *$ & $0.08^{*}$ & $0.07^{*}$ & 0.07 & $0.1^{*}$ & $0.09 *$ \\
\hline Rural & $0.06^{*}$ & $0.08^{*}$ & $0.07^{*}$ & -0.06 & 0.01 & $0.04^{*}$ \\
\hline ANC: +4 tetanus injection & $0.05^{*}$ & $0.03^{*}$ & $0.02 *$ & $0.23^{*}$ & $0.27^{*}$ & $0.22 *$ \\
\hline \multicolumn{7}{|l|}{ Age group } \\
\hline $15-24$ & $0.05^{*}$ & $0.04^{*}$ & $0.02 *$ & $0.24 *$ & $0.28 *$ & $0.22 *$ \\
\hline $25-49$ & $0.05^{*}$ & $0.03^{*}$ & $0.02 *$ & $0.23^{*}$ & $0.25 *$ & $0.22 *$ \\
\hline
\end{tabular}


Table 4.2 continued:

\begin{tabular}{|c|c|c|c|c|c|c|}
\hline \multirow[b]{2}{*}{ Service type / covariate } & \multicolumn{3}{|l|}{ Ghana } & \multicolumn{3}{|l|}{ Nigeria } \\
\hline & 2003 & 2008 & 2014 & 2003 & 2008 & 2013 \\
\hline \multicolumn{7}{|l|}{ Marital status } \\
\hline Never & 0.01 & $0.07^{*}$ & 0.02 & 0.08 & $0.1^{*}$ & $0.07^{*}$ \\
\hline Currently / previously & $0.05^{*}$ & $0.03 *$ & $0.02^{*}$ & $0.24^{*}$ & $0.27^{*}$ & $0.23^{*}$ \\
\hline \multicolumn{7}{|l|}{ Maternal occupation } \\
\hline Not working & 0.04 & $0.06^{*}$ & 0.01 & $0.33^{*}$ & $0.35^{*}$ & $0.29 *$ \\
\hline Professional / sales & $0.02 *$ & $0.02^{*}$ & 0.01 & $0.24^{*}$ & $0.23^{*}$ & $0.21^{*}$ \\
\hline Agriculture & $0.05^{*}$ & 0.02 & 0.01 & $0.14^{*}$ & $0.18^{*}$ & $0.1^{*}$ \\
\hline Others & $0.03^{*}$ & $0.03^{*}$ & 0.01 & $0.21 *$ & $0.27^{*}$ & $0.19 *$ \\
\hline \multicolumn{7}{|l|}{ Location } \\
\hline Urban & 0.01 & $0.02 *$ & 0.01 & $0.11^{*}$ & $0.09 *$ & $0.06^{*}$ \\
\hline Rural & $0.03^{*}$ & $0.03^{*}$ & $0.02^{*}$ & $0.2^{*}$ & $0.28^{*}$ & $0.23^{*}$ \\
\hline ANC: home & $-0.26 *$ & $-0.25^{*}$ & -0.1 & $-0.23^{*}$ & $-0.15^{*}$ & 0.05 \\
\hline \multicolumn{7}{|l|}{ Age group } \\
\hline $15-24$ & -0.07 & -0.23 & 0.15 & -0.23 & -0.08 & $0.21 *$ \\
\hline $25-49$ & $-0.3 *$ & -0.26 & -0.19 & $-0.24 *$ & $-0.17^{*}$ & 0.01 \\
\hline \multicolumn{7}{|l|}{ Marital status ${ }^{+}$} \\
\hline Never & 0.08 & - & -0.34 & -0.31 & -0.08 & -0.02 \\
\hline Currently / previously & $-0.27^{*}$ & - & -0.07 & $-0.22^{*}$ & $-0.16^{*}$ & 0.06 \\
\hline \multicolumn{7}{|l|}{ Maternal occupation ${ }^{+}$} \\
\hline Not working & -0.22 & - & 0.21 & -0.33 & $-0.22 *$ & 0.05 \\
\hline Professional / sales & -0.27 & - & -0.13 & -0.22 & $-0.14^{*}$ & 0.09 \\
\hline Agriculture & -0.24 & - & -0.06 & 0.05 & 0.01 & 0.11 \\
\hline Others & -0.43 & - & -0.53 & -0.24 & -0.07 & 0.13 \\
\hline \multicolumn{7}{|l|}{ Location $^{+}$} \\
\hline Urban & -0.41 & - & -0.18 & $-0.29 *$ & -0.13 & -0.02 \\
\hline Rural & -0.1 & - & 0.03 & -0.12 & $-0.09 *$ & 0.11 \\
\hline
\end{tabular}

Note: Magnitudes of regional variation is available in Appendix C8.

+ In 2008 the mean value of outcome is undefined.

Table 4.3 quantifies the degree of household wealth-related inequality in the use of delivery care services. Values of the concentration indices of home delivery declined in Ghana, increased in Nigeria, but remained negative in both countries. Closer observation reveals that the magnitude of inequality associated with the use of home delivery in Ghana was significant between regions in the years 2003 and 2014. Significant differences in the six regions of Nigeria were also observed, and point estimates show that the magnitude of inequality is not the same across geographical regions. In addition, results from Ghana data show the concentration of home delivery among poor households in rural and urban residences over the years. After 2008, there was no significant urban-rural differential in the magnitude of inequality in Ghana. However, in Nigeria, urban-rural inequality persisted. Though of equivalent magnitude, -0.09 , the tests of the null hypothesis of equality indicate a significant difference between women in rural and urban areas of Nigeria through the years. In 
2008, the concentration index magnitude in C-section increased in Nigeria from +0.49 to +0.58 but a decline in the concentration index magnitude was noted in Ghana from +0.45 to +0.30 for the same period. Significant differences between age groups were noted in Ghana and Nigeria after 2008; women who are 25-49 years consistently have greater concentration index magnitude in C-section.

Table 4.3: Concentration Indices with Covariates: Ghana (years 2000, 2005 \& 2014) and Nigeria (years 2003, 2008 \& 2013)

\begin{tabular}{|c|c|c|c|c|c|c|}
\hline \multirow[b]{2}{*}{ Service type / covariate } & \multicolumn{3}{|l|}{ Ghana } & \multicolumn{3}{|l|}{ Nigeria } \\
\hline & 2003 & 2008 & 2014 & 2003 & 2008 & 2013 \\
\hline Delivery: home & $-0.14^{*}$ & $-0.13^{*}$ & $-0.1^{*}$ & $-0.12^{*}$ & $-0.15^{*}$ & $-0.14^{*}$ \\
\hline \multicolumn{7}{|l|}{ Age group } \\
\hline $15-24$ & $-0.13^{*}$ & $-0.09 *$ & $-0.07^{*}$ & $-0.09 *$ & $-0.12^{*}$ & $-0.11^{*}$ \\
\hline $25-49$ & $-0.15^{*}$ & $-0.14^{*}$ & $-0.1 *$ & $-0.12^{*}$ & $-0.16^{*}$ & $-0.15^{*}$ \\
\hline \multicolumn{7}{|l|}{ Marital status } \\
\hline Never & $-0.1^{*}$ & $-0.1^{*}$ & $-0.07^{*}$ & $-0.09 *$ & $-0.09 *$ & $-0.09 *$ \\
\hline Currently / previously & $-0.14^{*}$ & $-0.13^{*}$ & $-0.1 *$ & $-0.11^{*}$ & $-0.15^{*}$ & $-0.14 *$ \\
\hline \multicolumn{7}{|l|}{ Maternal occupation } \\
\hline Not working & $-0.14^{*}$ & $-0.11^{*}$ & $-0.07^{*}$ & $-0.11^{*}$ & $-0.14^{*}$ & $-0.13^{*}$ \\
\hline Professional / sales & $-0.12^{*}$ & $-0.09 *$ & $-0.06^{*}$ & $-0.13^{*}$ & $-0.17^{*}$ & $-0.16^{*}$ \\
\hline Agriculture & $-0.06^{*}$ & $-0.09 *$ & $-0.06 *$ & $-0.08^{*}$ & $-0.08^{*}$ & $-0.06 *$ \\
\hline Others & $-0.13^{*}$ & $-0.1^{*}$ & $-0.08 *$ & $-0.11^{*}$ & $-0.17^{*}$ & $-0.15^{*}$ \\
\hline \multicolumn{7}{|l|}{ Location } \\
\hline Urban & $-0.07^{*}$ & $-0.05^{*}$ & $-0.04 *$ & $-0.09 *$ & $-0.12^{*}$ & $-0.09 *$ \\
\hline Rural & $-0.07^{*}$ & $-0.1^{*}$ & $-0.07^{*}$ & $-0.08^{*}$ & $-0.11^{*}$ & $-0.09 *$ \\
\hline Delivery: government health facility & $0.10 *$ & $0.10 *$ & $0.06^{*}$ & $0.05^{*}$ & $0.07^{*}$ & $0.08^{*}$ \\
\hline \multicolumn{7}{|l|}{ Age group } \\
\hline $15-24$ & $0.08 *$ & $0.07^{*}$ & $0.06^{*}$ & $0.04 *$ & $0.06^{*}$ & $0.07^{*}$ \\
\hline $25-49$ & $0.11 *$ & $0.11^{*}$ & $0.07^{*}$ & $0.06 *$ & $0.08^{*}$ & $0.08^{*}$ \\
\hline \multicolumn{7}{|l|}{ Marital status } \\
\hline Never & 0.04 & $0.1^{*}$ & $0.06 *$ & 0.01 & 0.03 & 0.03 \\
\hline Currently / previously & $0.11 *$ & $0.1^{*}$ & $0.07^{*}$ & $0.05^{*}$ & $0.07^{*}$ & $0.08^{*}$ \\
\hline \multicolumn{7}{|l|}{ Maternal occupation } \\
\hline Not working & $0.08 *$ & $0.06 *$ & $0.04 *$ & $0.06 *$ & $0.07^{*}$ & $0.08^{*}$ \\
\hline Professional / sales & $0.09 *$ & $0.07^{*}$ & $0.03^{*}$ & $0.05^{*}$ & $0.08^{*}$ & $0.08^{*}$ \\
\hline Agriculture & $0.04^{*}$ & $0.07^{*}$ & $0.06^{*}$ & 0.03 & $0.05^{*}$ & $0.04^{*}$ \\
\hline Others & $0.11^{*}$ & $0.07^{*}$ & $0.06^{*}$ & $0.05^{*}$ & $0.07^{*}$ & $0.07^{*}$ \\
\hline \multicolumn{7}{|l|}{ Location } \\
\hline Urban & $0.04 *$ & $0.03^{*}$ & 0.01 & 0.03 & $0.03 *$ & $0.03^{*}$ \\
\hline Rural & $0.05^{*}$ & $0.08 *$ & $0.06^{*}$ & $0.03 *$ & $0.06^{*}$ & $0.06 *$ \\
\hline Delivery: private health facility & $0.04 *$ & $0.03^{*}$ & $0.03^{*}$ & $0.06 *$ & $0.08^{*}$ & $0.07^{*}$ \\
\hline \multicolumn{7}{|l|}{ Age group } \\
\hline $15-24$ & $0.04^{*}$ & 0.02 & 0.01 & $0.05^{*}$ & $0.05^{*}$ & $0.05^{*}$ \\
\hline $25-49$ & $0.04^{*}$ & $0.03 *$ & $0.03 *$ & $0.07^{*}$ & $0.09 *$ & $0.07^{*}$ \\
\hline \multicolumn{7}{|l|}{ Marital status } \\
\hline Never & 0.06 & 0 & 0.01 & $0.09 *$ & $0.06 *$ & $0.05^{*}$ \\
\hline Currently / previously & $0.04 *$ & $0.03 *$ & $0.03 *$ & $0.06 *$ & $0.08^{*}$ & $0.07^{*}$ \\
\hline \multicolumn{7}{|l|}{ Maternal occupation } \\
\hline Not working & $0.06 *$ & $0.06 *$ & 0.03 & $0.06 *$ & $0.06 *$ & $0.05^{*}$ \\
\hline Professional / sales & $0.03 *$ & 0.02 & $0.04 *$ & $0.07^{*}$ & $0.09 *$ & $0.08^{*}$ \\
\hline Agriculture & $0.01^{*}$ & $0.02 *$ & 0 & $0.06^{*}$ & $0.03 *$ & 0.01 \\
\hline
\end{tabular}


Table 4.3 continued:

\begin{tabular}{|c|c|c|c|c|c|c|}
\hline \multirow[b]{2}{*}{ Service type / covariate } & \multicolumn{3}{|c|}{ Ghana } & \multicolumn{3}{|c|}{ Nigeria } \\
\hline & 2003 & 2008 & 2014 & 2003 & 2008 & 2013 \\
\hline Others & $0.02 *$ & 0.02 & 0.02 & $0.06 *$ & $0.09 *$ & $0.07^{*}$ \\
\hline \multicolumn{7}{|l|}{ Location } \\
\hline Urban & 0.03 & 0.01 & $0.03 *$ & $0.06 *$ & $0.09 *$ & $0.06 *$ \\
\hline Rural & $0.01^{*}$ & $0.02 *$ & 0.01 & $0.04 *$ & $0.04^{*}$ & $0.03 *$ \\
\hline Birth assistance: doctor & $0.03^{*}$ & $0.04 *$ & $0.05^{*}$ & $0.04 *$ & $0.06^{*}$ & $0.05^{*}$ \\
\hline \multicolumn{7}{|l|}{ Age group } \\
\hline $15-24$ & $0.03 *$ & 0.01 & 0.02 & $0.03 *$ & $0.03^{*}$ & $0.03 *$ \\
\hline $25-49$ & $0.04 *$ & $0.05^{*}$ & $0.06 *$ & $0.04 *$ & $0.06^{*}$ & $0.06 *$ \\
\hline \multicolumn{7}{|l|}{ Marital status } \\
\hline Never & 0.06 & 0.03 & $0.04 *$ & $0.11 *$ & $0.03^{*}$ & $0.03 *$ \\
\hline Currently / previously & $0.03^{*}$ & $0.04^{*}$ & $0.05^{*}$ & $0.03 *$ & $0.06^{*}$ & $0.05^{*}$ \\
\hline \multicolumn{7}{|l|}{ Maternal occupation } \\
\hline Not working & $0.05^{*}$ & $0.06 *$ & $0.03 *$ & $0.03 *$ & $0.05^{*}$ & $0.04 *$ \\
\hline Professional / sales & $0.04 *$ & $0.04 *$ & $0.06 *$ & $0.05^{*}$ & $0.07^{*}$ & $0.06 *$ \\
\hline Agriculture & $0.01^{*}$ & 0.01 & 0.01 & 0.04 & $0.01^{*}$ & $0.02 *$ \\
\hline Others & $0.04 *$ & $0.04 *$ & $0.06 *$ & $0.03 *$ & $0.06^{*}$ & $0.05^{*}$ \\
\hline \multicolumn{7}{|l|}{ Location } \\
\hline Urban & $0.04 *$ & $0.04 *$ & $0.05^{*}$ & $0.05^{*}$ & $0.08^{*}$ & $0.07^{*}$ \\
\hline Rural & 0.01 & $0.02 *$ & $0.03 *$ & $0.01^{*}$ & $0.02^{*}$ & $0.02 *$ \\
\hline C Section & $0.45^{*}$ & $0.30 *$ & $0.31^{*}$ & $0.49 *$ & $0.58^{*}$ & $0.49 *$ \\
\hline \multicolumn{7}{|l|}{ Age group } \\
\hline $15-24$ & 0.37 & 0.05 & 0.07 & 0.22 & $0.46^{*}$ & $0.33^{*}$ \\
\hline $25-49$ & $0.47^{*}$ & $0.34^{*}$ & $0.32 *$ & $0.54^{*}$ & $0.59 *$ & $0.52 *$ \\
\hline \multicolumn{7}{|l|}{ Marital status } \\
\hline Never & 0.38 & 0.26 & 0.2 & 0.53 & $0.57^{*}$ & 0.27 \\
\hline Currently / previously & $0.45^{*}$ & $0.31^{*}$ & $0.32^{*}$ & $0.48^{*}$ & $0.58^{*}$ & $0.5^{*}$ \\
\hline \multicolumn{7}{|l|}{ Maternal occupation } \\
\hline Not working & 0.36 & 0.26 & 0.15 & $0.5^{*}$ & $0.6^{*}$ & $0.59 *$ \\
\hline Professional / sales & $0.45^{*}$ & $0.19 *$ & $0.28 *$ & $0.44^{*}$ & $0.55^{*}$ & $0.49 *$ \\
\hline Agriculture & 0.14 & 0.28 & 0.11 & 0.61 & 0.19 & 0.28 \\
\hline Others & 0.43 & 0.25 & $0.27^{*}$ & 0.45 & $0.46^{*}$ & $0.43^{*}$ \\
\hline \multicolumn{7}{|l|}{ Location } \\
\hline Urban & $0.36 *$ & 0.11 & $0.19 *$ & $0.36^{*}$ & $0.41^{*}$ & $0.36^{*}$ \\
\hline Rural & 0.14 & $0.38^{*}$ & $0.3^{*}$ & 0.29 & $0.52^{*}$ & $0.37^{*}$ \\
\hline
\end{tabular}

Note: Magnitudes of regional variation is available in Appendix C9.

\subsection{DISCUSSION}

The concentration curve and associated indices estimated in this study permit the investigation of the progress made in reducing inequalities in access to reproductive health care services among women in Ghana and Nigeria. These indicators of use of family planning services, antenatal and delivery care services show that the use of some services is inequitably distributed and that there are differences in the size of inequality 
within socioeconomic groups of women. Specifically, the use of ANC at government facilities, health worker's assistance during pregnancy outside a facility, childbirth at home is distributed unequally and advantaging women in poor households, while the use of family planning information via TV or print media, ANC at private facilities are advantaging women in wealthier households in both Ghana and Nigeria. Only in Nigeria, women in richer households have considerably more unmet needs for family planning.

The variation in the magnitudes of inequality across socioeconomic groups within and between the countries (measured by the concentration indices), is also necessary to understand how the determinants of inequalities differ. We find that nearly all indicators of the use of reproductive health care services in Ghana indicated a shift towards the equity line, indicating a decline in inequality. However, equity improvement was not observed in doctor-assisted births, ANC provided at non-facility formations, government and private facilities. In Nigeria, indicators examined showed mixed shifts, with mostly non-pro-poor changes over the years observed. This was specifically the case for ANC at private facilities, ANC in government facilities, non-facility formations for ANC, family planning information via TV or print media, unmet needs for family planning, and childbirth at home. The most substantial change in the magnitude of inequality, a decrease, was noted for the use of modern contraceptives during the years 2003 - 2008 in both Ghana and Nigeria. The most substantial change in ANC was observed in 2003 - 2008 for the indicators ANC at government health facilities in Ghana and ANC in the 1st trimester in Nigeria. Among indicators of delivery care, the most substantial change in the magnitude of inequality is home delivery in Ghana occurred among women in poor households in the period 2008 - 2014. In Nigeria, a change in the magnitude of inequality was most evident in delivery at a government health facility and private health facility during the period 2003-2008.

\subsubsection{Family planning services}

We find that the use of family planning information via TV or print media is unequally distributed in favor of women in wealthy households of Ghana, specifically, those with agricultural livelihood and women who live in the Upper East region. This is unsurprising since wealth is correlated with education which facilitates access and assimilation of information (Solar \& Irwin, 2010). This finding supports previous research that showed that higher socioeconomic status improves the use of family planning media messages (Kwankye \& Augustt, 2013). Although studies show that few people get family planning information via media messages (Onwuzurike \& Uzochukwu, 2001). Another study found that access to family planning information via television or print medium 
is in disfavor of women in lower socioeconomic strata (Ajaero, Odimegwu, Ajaero, \& Nwachukwu, 2016). However, access to family planning information via media messages about reproductive health care services has mixed results in promoting access in Africa (Ajaero et al., 2016; Awoonor-Williams et al., 2013; Gupta, Katende, $\&$ Bessinger, 2003). We also find that unwanted births, indicated by unmet needs for family planning, are concentrated in wealthy households of Nigeria and occur most among women who are not working or living in rural residences. This finding suggests that there is a high need for contraception among women in rural economically advantaged households. Studies show that wage-earning or economically selfsufficient women are more likely to seek contraception, though modern means of preventing unwanted births could be inaccessible in cultural and religious societies (Onwuhafua, Kantiok, Olafimihan, \& Shittu, 2005; Onwuzurike \& Uzochukwu, 2001). Finally, regarding access to family planning services, while economic status does preclude women from making sole reproductive decisions it could, however, initiate a demand for contraception (Crissman, Adanu, \& Harlow, 2012; Gakidou \& Vayena, 2007; Omeje, Oshi, \& Oshi, 2011).

\subsubsection{ANC services}

We find that, in both Ghana and Nigeria, women in poor households have increasingly become disadvantaged as inequality in the use of antenatal care at private facilities increased in favor of their counterparts in wealthy households. A study carried out in Ghana using DHS data from years 1988 - 2008 found a similar increase. However, this study did not disaggregate antenatal care by type of provider (Asamoah et al., 2014). It is reported that wealthier women are better able to overcome barriers of informal payments of cash or kind and are less likely to encounter negative health workers' attitudes often seen in private health care facilities (Onwujekwe, Onoka, Uzochukwu, \& Hanson, 2011; Pell et al., 2013). Other studies have shown that wealth-related inequalities in the use of antenatal care have increased in the past years (Asamoah et al., 2014; Obiyan \& Kumar, 2015). Results from our study further suggest that the use of health worker's assistance during pregnancy outside a facility in both countries became less equitable; women in poor households in urban areas or with professional/sales occupation use such assistance more frequently. Professional occupation and urban area residents are generally thought to have better access to good quality reproductive health care services, given their knowledge and the service availability accessible to these women to draw from (Aremu, Lawoko, \& Dalal, 2011; Ayanore, Pavlova, \& Groot, 2016a; Ochako et al., 2011; Onah et al., 2006). Our finding deviates from other studies that suggest that women in these groups access better antenatal care services. To explain the variation in the use of health worker's assistance during pregnancy outside 
a facility, studies have also shown that transport and health facilities waiting time may facilitate the use of such assistance or deter the use of modern antenatal care services among women in these categories (Arthur, 2012; Ayanore et al., 2016a; Fagbamigbe \& Idemudia, 2015; Jallow, Chou, Liu, \& Huang, 2012; Mrisho et al., 2009). Specifically, the observed change in home antenatal care from more prominence among the poor to more prominence among the richer households in Nigeria is unexpected. Fagbamigbe and Idemudia (2017) also noted non-use of antenatal care among the wealthier women during pregnancy and suggest that not only poverty but also other factors like personality and the perception on the quality of services are relevant. It is also plausible that these are a response to the increased pressure on resources in government or other maternal care formations (Mrisho et al., 2009). Unfortunately, there is no information on the quality of care in the DHS data.

The finding that antenatal care in government facilities in both countries is propoor and consistently changing to the advantage of women in poor households was observed in different years. No effect was observed among women in the agrarian sector, however. One study reported that the use of antenatal care improved among women in Ghana, and, though economic challenges are being surmounted, it may be delayed among women in agricultural occupations (Asamoah et al., 2014). Other studies report pro-wealthy inequality changes in antenatal care use among women in Ghana and Nigeria between 2003 and 2008 (Johnson et al., 2016; Obiyan \& Kumar, 2015). In addition, the urban-rural inequality in the use of antenatal care at government facilities was observed in the later years in Nigeria but diminished in Ghana. In Nigeria, we find no evidence that rural women in poor households seek antenatal health care services at government facilities. Other studies found unequal use of antenatal care services to the detriment of women in rural households (Fagbamigbe $\&$ Idemudia, 2015, 2017). A study of Nigeria's midwives service scheme found insignificant success in rural areas attributable to pro-wealthy resource distribution (Okeke et al., 2015). Nonetheless, the observed diminished urban-rural differential to benefit women in poor households in Ghana has been partially credited to improvements in infrastructure and maternal health care services (Asamoah et al., 2014).

\subsubsection{Delivery care services}

Our study finds that childbirth at home persists among women in poor households although overall, the inequality magnitude appears to have declined in Ghana while it has increased in Nigeria, and there are substantial geographical variations. It appears that by 2014 inequality became notable among women in all seven regions of Ghana. In a 2005 research on the free delivery care policy in Central and Volta regions of 
Ghana, an increase in facility delivery and a decline in home delivery was reported (Asante et al., 2007). Another study found that coverage of the doorstep communitybased health planning and services program in Ghana was substantial in mainly the Upper East region (Awoonor-Williams et al., 2013). A separate study carried out among Nigerian women in 2004 did not find a substantial increase in institutional delivery facilities in Nigeria despite the midwives service scheme (Okeke et al., 2015). Evidence of substantial pro-rich inequality between the Northern and Southern regions was observed in Nigeria, while delivery at government health facilities favored women in the Northern regions. Delivery at a private health facility is more inequitable among the richer households in the Southern regions. We find persisting rural-urban disparities associated with childbirth at a government health facility in Nigeria, but not in the later years in Ghana, which is 2008 and 2014. Other research did not find evidence of rural-urban differences in the shift from home to health facilities in Ghana and Nigeria (Amoako Johnson et al., 2013). The observed inequalities among women who have childbirth at home suggest that health policies such as the community-based health planning and services initiative in Ghana, a free delivery scheme in Ghana, the midwives service scheme in Nigeria and insurance schemes in both countries have not substantially reduced inequality in home birth among women. This is also confirmed in other studies (Asamoah et al., 2014; Obiyan \& Kumar, 2015; Zere et al., 2012).

The finding that $\mathrm{C}$-section in both Ghana and Nigeria are pro-wealthy is not surprising. Our findings are in line with the findings of previous studies that $\mathrm{C}$-sections remain under-provided for women in poor households in both countries (Zere et al., 2012). The trend suggests that the coverage gap in both countries remains relatively high. In addition, the equity trend observed in Nigeria suggests top inequity, indicating that the increase in the concentration index magnitude related to $\mathrm{C}$-section is extremely high.

\section{Study limitations}

The cross-sectional design of the study implies that we can show associations without concluding about causal relationships. Other methodological limitations of the study include recall bias since the survey collects events over a five-year period. A limitation regarding the country comparisons concerns the fact that inequalities were investigated based on the position of women in the distribution of household wealth in their own country. We recognize that a woman who is poor by Ghana standards may be better off in Nigeria. Also, we recognize that measures of coverage gap (Countdown Equity Analysis et al., 2008), which we have not analyzed, are as equally important as the equity gap evidenced in this chapter. The coverage gap refers to the difference between the targeted and actual use of essential health care services by 
the population, while the equity gap indicates the distribution of service use across the wealth-based population groups (Countdown Equity Analysis et al., 2008). Thus, the underutilization of reproductive care is not directly addressed in our equity study. However, the study has some strengths as well. In particular, the merging of important indicators of maternal care improved the ability to capture the different categories of reproductive care. In addition, we use a generalized concentration index as a measure of inequality, which is not sensitive to outcome measures because it quantifies the absolute differences in health between income groups. Finally, the measurement of the magnitudes of inequalities over different years gives indications about the changing horizontal inequalities which are country and time-specific.

\subsection{CONCLUSION}

Inequality in the use of family planning, antenatal care and delivery care among women in both Ghana and Nigeria have persisted over the years despite efforts and have provided little improvement for women in poor households. The results show that inequality increased in the case of ANC at private facilities, health worker's assistance during pregnancy outside a facility, ANC in government facilities, home births, aspects of reproductive health care services in both Ghana and Nigeria, and unmet need for family planning in Nigeria. Changes in inequality were mostly to the disadvantage of women in poorer households in Nigeria but less in Ghana. The changes in inequality had little effect on improving the use of quality reproductive health care services among women particularly those in poor households. Furthermore, the disambiguation of indicators of the use of reproductive health care services shows the extent of the progress made in eliminating unequal access among sociodemographic groups. Also, disaggregation of determinants of access indicated notable horizontal inequalities among women of different socioeconomic groups in Ghana and Nigeria.

The gains made in reducing inequality access to reproductive health care services have eroded over time. This implies that the sustainability of health initiatives to reduce inequalities needs to be addressed. Ghana's health initiatives need to take a pro-poor concept and Nigeria's accelerated implementation across the population to bring about the decline in inequality in access. 
EXAMINING TRENDS IN INEQUALITY IN THE USE OF REPRODUCTIVE HEALTH CARE SERVICES IN GHANA AND NIGERIA 



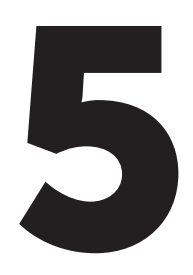

INEQUALITIES IN REPRODUCTIVE HEALTH CARE USE IN FIVE WEST AFRICAN COUNTRIES: DECOMPOSITION ANALYSIS OF THE WEALTH-BASED GAPS IN BURKINA FASO, GHANA, NIGER, NIGERIA, SENEGAL

\section{Draws upon:}

Ogundele, O. J., Pavlova, M., \& Groot, W. (2020). Inequalities in reproductive health care use in five West-African countries: A decomposition analysis of the wealth-based gaps. International Journal for Equity in Health, 19(1), 44. 


\section{ABSTRACT}

\section{Background}

Family planning and maternal care services have become increasingly available in West Africa but the level of non-use remains high. This unfavorable outcome may be partly due to the unaffordability of reproductive health care services.

\section{Methods}

Using the DHS data from Burkina Faso, Niger, Nigeria, Ghana, and Senegal, a decomposition analysis was performed to quantify the contribution of sociodemographic characteristics to disparities in the exposure to mass media information on family planning, use of modern contraceptives, adequate ANC visits, facility-based childbirth and C-section between low-wealth and high-wealth women.

\section{Results}

Our study shows that differences in maternal characteristics between the wealth groups explain at least $40 \%$ of the gap in exposure to mass media family planning information, $30 \%$ in modern contraceptive use, $24 \%$ of adequate ANC visits, $47 \%$ of the difference in facility-based childbirths, and $62 \%$ in C-section. Lack of information on pregnancy complications, living in rural residence, religion, lack of autonomy in health facility seeking decision, need to pay, and distance explains the disparity in reproductive health care use across all countries. In countries with complete fee exemption policies for specific groups in the population, Ghana, Niger, and Senegal, the inequality gaps between wealth groups in having an adequate number of ANC visits and facility-based childbirth are smaller than in countries with partial or no exemption policies. But this is not the case for $\mathrm{C}$-section.

\section{Conclusion}

There is evidence that current policies addressing the cost of maternal care services may increase the wealth-based inequality in maternal care use if sociodemographic differences are not addressed. Public health interventions are needed to target sociodemographic disparities and health facility seeking problems that disadvantage women in poor households. 


\subsection{BACKGROUND}

Many low- and middle-income countries have made efforts to foster equal access to health care. These efforts have not attained similar results (WHO, 2015b). In 2015, the World Health Organization and UNICEF reported differences in maternal health outcomes between low- and middle-income countries, with maternal deaths ranging between 599 and 849 per 100,000 live births in West and Central Africa combined. Tsui, Brown, and Li (2017) also provide evidence of the differences in the use of modern contraceptives within and across sub-Saharan African countries. For example, the prevalence of modern contraceptives is $18.5 \%$ in Kaduna, Nigeria and $26.4 \%$ in Lagos, Nigeria. The observed disparities in reproductive health outcomes and utilization are partly attributable to wealth. Evidence shows that the most vulnerable - the poor - are at a disadvantage, which prevents equal access to care (Alam, Hajizadeh, Dumont, \& Fournier, 2015; Creanga, Gillespie, Karklins, \& Tsui, 2011; Gakidou \& Vayena, 2007; Peters et al., 2008).

There is evidence to suggest that the poor have peculiar characteristics, e.g. illiteracy, high parity and inefficient and insufficient exposure to the reproductive health information on mass media, which stymie their use of health care services in general (Abekah-Nkrumah, 2018; Ahmed et al., 2010; Creanga et al., 2011; Gillespie, 2007; Goli, Doshi, \& Perianayagam, 2013; Johnson et al., 2016). This applies to the use of reproductive health care services as well (see Chapter 1). Several studies have shown that better-off women have easier access to reproductive health care (Alam et al., 2015; Do et al., 2015; Goli, Nawal, Rammohan, Sekher, \& Singh, 2018; Hosseinpoor et al., 2011; Ravit et al., 2018). In Chapter 4, sociodemographic factors such as marital status, education, occupation, residence typology, attitude of health workers, and distance to health facility also contribute to the inequality in the use reproductive health care among women. In addition, it is unclear to what extent these determinants explain the use of reproductive health care and contribute to the differences between the groups (Abekah-Nkrumah, 2018; Kendall \& Langer, 2015). In view of this evidence, a policy that aims to reduce inequality and helps to attain universal coverage of reproductive health care services should target the poorest people most in need of health care but should also take into account other determinants than wealth.

This study focuses on the gaps in the use of reproductive health care in five countries in West Africa, namely Burkina Faso, Ghana, Niger, Nigeria, and Senegal. These countries have a relatively high maternal mortality rate - in the range of 315-814 per 100000 live births (WHO, 2015b). In addition, the countries have similar policies, either in place 
or in a process of implementation, to address reproductive health care services as part of the efforts to reduce maternal mortality as mentioned in Chapter 1. Burkina Faso is a low-income country with an estimated 18.6 million people in 2016 (WHO, 2020a). The gross national income per capita in 2013 was US\$1,560. The maternal mortality ratio was estimated at 371 per 100,000 live births in 2015. Burkina Faso has a national maternal health care subsidy policy (Richard et al., 2013; Ridde et al., 2012). The general government expenditure on health as a percentage of total government expenditure in 2014 was $11.2 \%$ and private expenditure on health was $47.7 \%$ of total expenditure on health (Global Health Observatory, 2020).

Niger is a low-income country with an estimated population of 20.6 million and a gross national income per capita of $\$ 910$ in 2013 (WHO, 2020c). The estimated maternal mortality ratio in Niger in 2010 was 590 per 100,000 live births. Niger implemented a user fees abolition policy for antenatal care, Caesarean sections and family planning as early as 2007 (Ridde \& Diarra, 2009). Niger's general government expenditure on health as a percentage of total government expenditure was $11.1 \%$ and private expenditure on health as a percentage of total expenditure on health was 44.9\% in 2011 (Global Health Observatory, 2020).

Nigeria's health care context has been described in more detail in Chapter 1. To recap, Nigeria is classified as a lower-middle-income country. It has an estimated population of 186 million in 2016 and gross national income per capita in 2013 was US\$5,360 (WHO, 2020d). The maternal mortality ratio in Nigeria was 814 per 100,000 live births in 2015 (WHO, 2020d). Nigeria has user fees but there are intermittent partially free maternal health care programs and a countrywide midwives scheme to improve the use of maternal health services (Edu et al., 2017; Mohammed \& Dong, 2012; Richard et al., 2013). The total expenditure on health per capita in 2014 was US\$216.87. General government expenditure on health as a percentage of total government expenditure was $8.2 \%$ and the private expenditure on health as a percentage of total expenditure on health was 74.9\% (Global Health Observatory, 2020).

Ghana's health care context has been described in Chapter 1 as well. Ghana is a lowermiddle-income country with a population estimate of 28.2 million in 2016 and a gross national income per capita of US\$3,880 in 2013 (WHO, 2020b). The maternal mortality ratio in Ghana was 319 per 100,000 live births in 2015. In Ghana, antenatal care in all public health facilities is for free since 1998. Aa free delivery care policy initially in four regions (northern, upper east, upper west and central) was extended to all regions in 2005 (Witter et al., 2007). The government of Ghana's general expenditure on health 
as a percentage of total government expenditure was $6.8 \%$ in 2014 while private expenditure on health as a percentage of total expenditure on health accounted for 40.2\% (Global Health Observatory, 2020).

Senegal is another lower-middle-income country with an estimated population of 15.4 million in 2016, and gross national income per capita US\$2,240 in 2013 (WHO, 2020e). The maternal mortality ratio was approximately 315 per 100,000 live births in 2015. Senegal has the free childbirth care and C-section policy (Witter et al., 2010). The general government expenditure on health as a percentage of total government expenditure was $8.0 \%$ and private expenditure on health as a percentage of total expenditure on health accounted for 48.2\% in 2014 (Global Health Observatory, 2020). This study investigates the wealth-based inequalities in the use of reproductive health care services among women in the selected countries and identifies factors that contribute to these inequalities. In particular, the probability of the use of reproductive health care services is decomposed for low-wealth and high-wealth women within the countries, and the relative disadvantage of different factors for the low-wealth group is analyzed. Sociodemographic, health and wealth contributing factors are included. We provide new knowledge by identifying characteristics that widen or narrow the inequality gap in reproductive health use. The study contributes to our understanding of the vulnerable groups that could be targeted in programs to "close the gap" between rich and poor. We also add to the current literature by presenting a cross-country comparison based on nationally representative datasets. The results may also be of interest to other low-middle income countries where reproductive health care use is on the policy agenda.

\subsection{METHODS}

\subsubsection{Data}

Data from the DHS were used. DHS are cross-sectional, comparable and representative population-based surveys that gather varied information on reproductive health, nutrition and other demographics of respondents from low-middle income countries (DHS, 2018). We used the latest DHS surveys available for Burkina Faso (2010), Niger (2012), Nigeria (2013), Ghana (2014) and Senegal (2016 DHS-VII). Details of the DHS survey methods are presented in the dataset section of Chapter 1.

We only used data provided by women of reproductive age (15-49 years) who had given birth during the last 5 years before the survey and who were able to provide 
information on the use of reproductive health care services. Appendix D1 summarizes the sample statistics of selected variables by wealth group.

\subsubsection{Variables}

The primary outcome variables of interest are binary indicators of whether the woman was exposed to family planning information via mass media, used modern contraceptives, made four or more ANC visits when pregnant, had facility-based childbirth, and had a C-section childbirth. All five variables were dichotomized as 1 when a woman answered "Yes" to the respective question, and otherwise as 0 "No".

We used the wealth index constructed by the DHS. This index is based on available information about household asset ownership, and housing and environmental conditions to analyze inequalities in reproductive health care services (Rutstein \& Johnson, 2004). We dichotomized the wealth quintile index where low wealth represents the lowest quintile and high wealth represents women in the higher four wealth quintiles.

The sociodemographic covariates included were selected based on findings from Chapter 4 and previous empirical research on inequality and access to reproductive health care services (Adeyanju, Tubeuf, \& Ensor, 2017; Alam et al., 2015; Asamoah et al., 2014; Do et al., 2015; Mwase et al., 2018; Rossier et al., 2014). For independent variable with more than $5 \%$ missing cases, we added a separate dummy variable indicating a missing value in the analysis. Details of covariates included in the analysis are presented in Appendix D2.

\subsubsection{Statistical analysis}

To determine the extent to which wealth-based differences in exposure to mass media family planning information, use of modern contraceptives, adequate use of ANC visits, use of facility-based childbirth services and $\mathrm{C}$-sections are due to differences in the observed respondents' characteristics, we used a non-linear decomposition technique suggested by Fairlie (Fairlie, 1999). The Fairlie decomposition technique is an extension of the Blinder-Oaxaca decomposition technique to binary outcome measures (Fairlie, 2006; Fairlie, 1999). Fairlie decomposition has been used to study group differences in a binary outcome variable including group differences in health facility utilization, racial differences in health outcome, rural-urban inequality, the disparity in health care utilization and insurance (Abekah-Nkrumah, 2018; Brick, Layte, Nolan, \& Turner, 2016; Liao, Chang, Wang, \& Sun, 2016; Liu, Gao, \& Yan, 2014). The Fairlie decomposition generates simulated samples of data that pair observations (a one-to-one matching) 
from each wealth group and estimates the predicted differences between those samples (Fairlie, 1999). The average contribution of each variable to the estimated gap from all iterated samples is reported as output (Fairlie, 2006).

The average difference in the use of a reproductive health care service between the wealth groups can be expressed as:

$$
\bar{Y}^{l w}-\bar{Y}^{h w}=\left[\sum_{i=1}^{N^{l w}} \frac{F\left(X_{i}^{l w} \hat{\beta}^{l w}\right)}{N^{l w}}-\sum_{i=1}^{N^{h w}} \frac{F\left(X_{i}^{h w} \hat{\beta}^{l w}\right)}{N^{h w}}\right]+\left[\sum_{i=1}^{N^{h w}} \frac{F\left(X_{i}^{h w} \hat{\beta}^{l w}\right)}{N^{h w}}-\sum_{i=1}^{N^{h w} F\left(X_{i}^{h w} \hat{\beta}^{h w}\right)} N^{h w}\right.
$$

where $\bar{Y}$ is the average probability of use of a reproductive health care service by a group (a dummy variable which, depending on the analysis, includes whether a woman had at least one family planning information source, used modern contraceptives, 4+ ANC visits, facility-based childbirth, C-section respectively), (windicates thelowwealth group and $h w$ indicates the high-wealth group. $X_{i}$ is the vector of independent variables of respondent $i$ in a given group (low-wealth or high-wealth group), $\hat{\beta}$ is the vector of coefficients estimated separately for each of the two groups using the pooled sample, $N$ is the number of observations in a given group, and $F$ is the cumulative logistic distribution function.

The first term on the right-hand side of equation (5.1) measures the proportion of the wealth gap in the use of a selected reproductive health care service that is due to differences in the distribution of characteristics of the two groups. This is the variation explained by differences in observable characteristics. This represents the extent to which the wealth gap in reproductive health care services would reduce if the lowwealth group had the same characteristics as the high-wealth group. The second term captures the degree to which women in the low-wealth and high-wealth groups, with similar observable characteristics, have different propensities in use. This indicates the portion of the gap that is due to differences in the impact of observable characteristics between the low- and high-wealth groups. This is the portion of the gap that may be due to wealth discrimination, differences in the availability of health care services, differences in attitudes between wealth groups, or other unmeasured characteristics (Fairlie, 2006).

Using the fairlie command in STATA 15, we draw a random subsample of the highwealth group equal to the size of the low-wealth group with randomly ordered variables and 1,000 replications to decompose the explained part and to show the contribution of each of the variables to the gap. Using the high-wealth group 
subsample as the reference category, we report the results of the contribution of maternal characteristics to the gap in the outcome of reproductive health care services observed. We performed a sensitivity analysis by using the coefficients of the upper three quintiles against the lower two quintiles. The results (not shown) are generally similar across specifications.

\subsection{RESULTS}

Table 5.1 shows the sample characteristics of respondents included in the analyses for each country. Of particular note is the proportion in the low-wealth group that has no education in Burkina Faso, Niger and Senegal, accounting for more than $80 \%$ of the respondents. More women in the high-wealth group reside in urban residences in Nigeria, Ghana, Senegal (at least 45\%) compared with Burkina Faso and Niger (at most $23 \%)$. Also, more women live in rural locations in Niger and Burkina Faso compared with Ghana, Senegal, and Nigeria. On average, women are unlikely to have information on pregnancy complications in Burkina Faso, Niger, and Senegal. On average, women who needed permission to visit health facilities or who reported problems with money to pay, were more likely to be in Burkina Faso and Niger compared with Nigeria and Ghana. Although the problem of money to pay among women is comparable among women in the low-wealth group in Nigeria and Ghana. Generally, women are less likely to report having a problem to visit a health facility alone across all countries. Although descriptive, the statistics in Table 5.1 highlight some significant differences between women in different countries which may have an effect on use of reproductive health care services. See Supplementary Appendix D1 for regional covariates included in the analysis. In the analysis, we included sampling weights to ensure populationrepresentative analysis.

\subsubsection{The wealth-based trend in the probability of the use of reproductive health care services}

There were substantial differences between low-wealth and high-wealth women in the exposure to mass media information on family planning, the use of modern contraceptives, adequate ANC visits, and the use of facility-based childbirth services, and C-section at childbirth (see Figure 5.1). On average, 50\% of women in the lowwealth group in Burkina Faso were exposed to mass media family planning information, while in other countries this was less, e.g. only $10 \%$ in Nigeria. For the high-wealth category, at least $52 \%$ of women were exposed to family planning information, except in Nigeria (40\%). The proportion of modern contraceptives use among women in the 


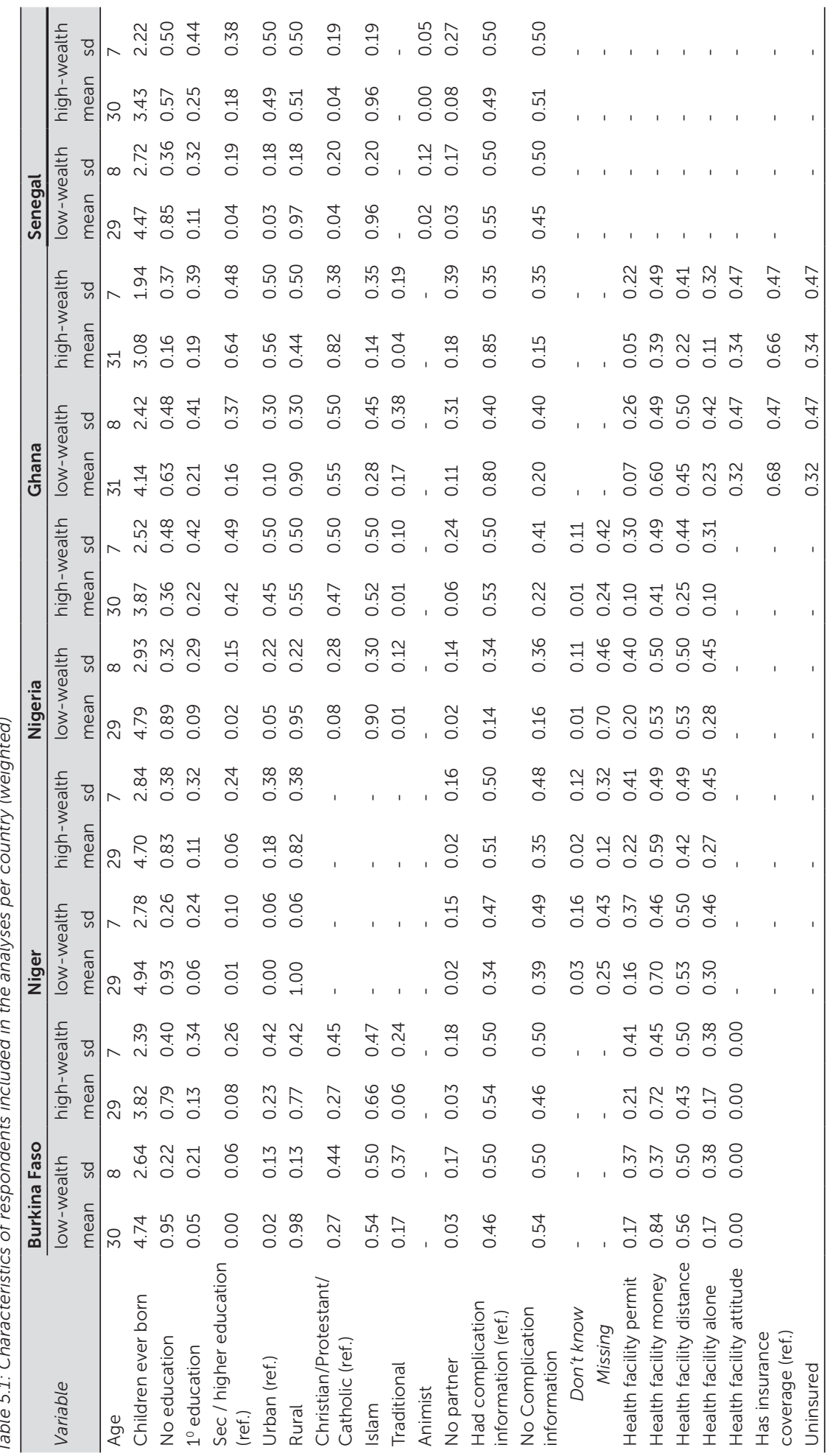


low-wealth group was generally low, namely $1 \%$ in Nigeria, $7 \%$ in Burkina Faso and $21 \%$ in Ghana, relative to $13 \%, 17 \% 26 \%$ among high-wealth group women in the same countries respectively. The wealth-based gap in the proportion of use of modern contraceptives was highest in Senegal - about 16 percentage points. Except for Ghana, the proportion with adequate ANC visits in the low-wealth group was relatively small; $18 \%$ in Nigeria, 24\% in Niger and Burkina Faso, 34\% in Senegal. The observed proportion of adequate visits among women in the high-wealth group ranged from 35\% in Niger to $91 \%$ in Ghana. Among the low-wealth group, only $5 \%$ of women in Nigeria had facility-based childbirth, $15 \%$ in Niger and over $45 \%$ in the other countries. In the high-wealth category, at least $77 \%$ of women in Burkina Faso, Ghana, and Senegal had facility-based childbirth while Nigeria and Niger had lower proportions. About $1 \%$ of women in the low-wealth group living in Nigeria had a C-section compared with $21 \%$ of women in Ghana. In other countries, this ranged from $7 \%$ to $14 \%$. On the other hand, of the women in the high-wealth group, 13\% in Nigeria had a C-section birth, $30 \%$ in Senegal and $26 \%$ in Ghana.

As indicated above, there are substantial differences in the use of maternal health services between the five countries included in our study.

\subsubsection{Decomposition results}

Table 5.2, 5.3, 5.4, 5.5 and 5.6 present the results of the decomposition analyses of exposure to family planning information via mass media, modern contraceptive use, adequate ANC visits, facility-based childbirth and C-sections (the outcome variables) among women in the low-wealth group and high-wealth group. In the upper part of these tables, the results of the probability of use, the difference or wealth gap, and the portion of that gap explained by observable characteristics are shown. The "explained" part is the proportion of the difference explained by the characteristics of women included in the analysis. If the low-wealth and high-wealth women had the same characteristics, then the "explained" portion would reduce the wealth-based gap in the outcome variable of interest. The lower part of these tables show the estimates of the contribution of sociodemographic characteristics to the explained portion of the gap. Negative estimates indicate that wealth differences in the independent variable increase the wealth gap (i.e. reduce the probability) in the use of reproductive health care. A positive estimate of the decomposition indicates the opposite. The key findings of the results are highlighted. 

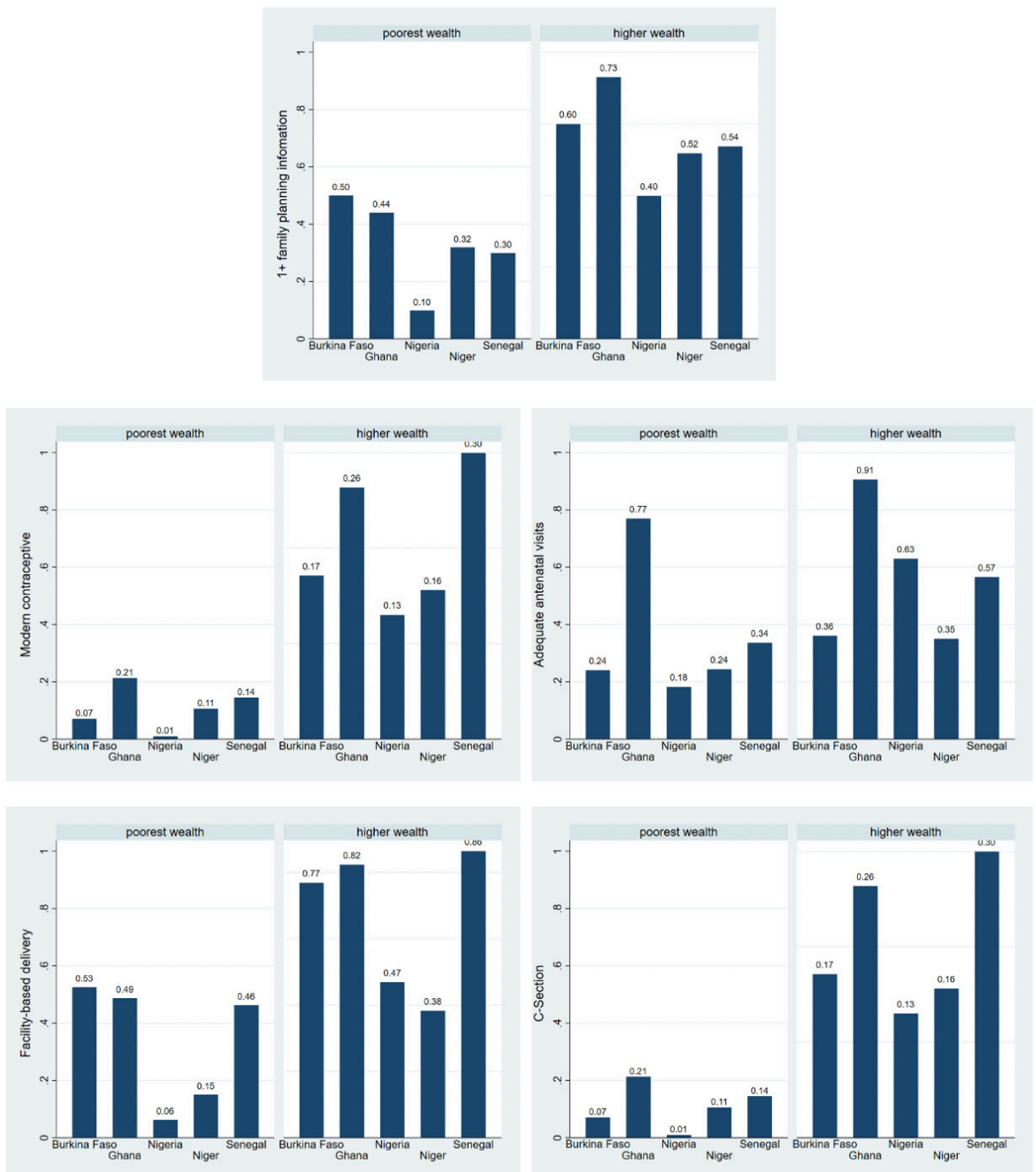

Figure 5. 1: Mean of reproductive health care service use in the countries, by wealth category (weighted)

\section{Exposure to family planning information via mass media}

Table 5.2 shows that the wealth-based gap in the probability of exposure to mass media messages was largest in Nigeria (0.302) and smallest in Burkina Faso (0.107). The included variables explain about 80.2\% (Nigeria), 66\% (Ghana), 58\% (Senegal), $51.8 \%$ (Niger) and 40\% (Burkina Faso) of this gap. The contribution of the number of children ever born by a woman to the gap was significant in Burkina Faso and Senegal and contributed about $18 \%$ and $9 \%$ respectively. Money problems in visiting 
a health facility significantly increased the gap in Burkina Faso, Ghana, and Nigeria respectively. Distance problems contributed to the gap in Burkina Faso and Niger. Getting permission to get medical help for self and attitude of health facility workers increased the wealth gap only in Nigeria. Lack of pregnancy complication information increased the gap by as much as 32\% in Niger, 22\% in Nigeria, but decreased the gap in Senegal 3\%. The Centre region of Burkina Faso, the Tillaberi in Niger, North East in Nigeria, Upper East in Ghana, and Tambacounda region. Senegal has the largest contribution to the wealth gaps observed.

\section{Modern contraceptive use}

Table 5.3 indicates that the wealth-based gap in the probability of modern contraceptive use was largest in Senegal (0.154) followed by Nigeria (0.121) and lowest in Burkina Faso (0.099) while Niger and Ghana had similar wealth gaps of 0.050 and 0.049 respectively. The disparity in modern contraceptive use explained by observable characteristics was about $88 \%$ of the wealth gap in Nigeria, $60 \%$ in Burkina Faso, $58 \%$ in Senegal, 30\% in Ghana. The observed characteristics explained the entire low/highwealth gap in modern contraceptive use in Niger. Woman's number of children ever born reduced the wealth gap in the use of modern contraceptives in all countries. Of the problems associated with seeking care at a health facility, the distance was most prominent and had the effect of increasing the wealth gap in the probability of use of modern contraceptives in Ghana by around 62\% and less in Senegal. For health facility, money problems explained the wealth gap in Burkina Faso and permission to get medical help for self only in Nigeria. Regions that considerably increased the wealth gaps were Hauts Basins in Burkina Faso, Tahoua in Niger, North East region in Nigeria, Northern region in Ghana as well as Kolda region in Senegal. None of the regions in Niger reduced the wealth gap in modern contraceptives use.

\section{Adequate ANC visits}

Table 5.4 shows that low-wealth women in Burkina Faso (0.268), Niger (0.244), Nigeria (0.183) and Senegal (0.369) have relatively little chances of receiving an adequate number of antenatal visits. But relatively higher in Ghana (0.816). The wealth-based gap in the probability of having adequate antenatal visits was 0.206 in Senegal and 0.447 in Nigeria. Of this disparity in an adequate number of antenatal visits, characteristics included in the model explained as little as 25\% in Niger and as much as $91 \%$ in Ghana. In Burkina Faso, Senegal, and Nigeria, the explained portion was $64 \%$,62\%, and $40 \%$ respectively. The number of children ever born by a woman increased the wealth gap by not less than $5 \%$ in all five countries. Regarding health facility seeking problems, the problem of permission to get medical help self significantly increased the wealth- 
based gap in adequate antenatal care use in Burkina Faso only. Money problems that prevent a health facility visit explained the wealth gap in having an adequate number of antenatal care visits in Nigeria, distance problems explained the wealth gap in Burkina Faso and Nigeria, while the problem of going alone to a health facility did so only in Ghana. Regional contributions to increased disparities were explained by seven regions in Senegal (mostly the Kolda region), and five in Burkina Faso (mostly the Sahel region). Two regions in Nigeria and one region in both Niger and Ghana increased the wealthgap in adequate antenatal care visits.

Table 5.2: Decomposition of exposure to family planning information media source

\begin{tabular}{|c|c|c|c|c|c|c|c|c|c|c|}
\hline \multicolumn{11}{|c|}{ Exposure to family planning information media source } \\
\hline & \multicolumn{2}{|l|}{ Burkina Faso } & \multicolumn{2}{|l|}{ Niger } & \multicolumn{2}{|l|}{ Nigeria } & \multicolumn{2}{|l|}{ Ghana } & \multicolumn{2}{|l|}{ Senegal } \\
\hline $\operatorname{Pr}$ (low-wealth) & 0.498 & & 0.318 & & 0.096 & & 0.450 & & 0.308 & \\
\hline Pr(high-wealth) & 0.604 & & 0.517 & & 0.399 & & 0.734 & & 0.539 & \\
\hline Difference & -0.107 & & -0.199 & & -0.302 & & -0.285 & & -0.231 & \\
\hline Total explained & -0.043 & & -0.103 & & -0.242 & & -0.190 & & -0.135 & \\
\hline$\%$ explained & & $40.2 \%$ & & $51.8 \%$ & & $80.2 \%$ & & $66.7 \%$ & & $58.4 \%$ \\
\hline \multicolumn{11}{|l|}{ Variable } \\
\hline contribution & Decomp. & $\%$ & Decomp. & $\%$ & Decomp. & $\%$ & Decomp. & $\%$ & Decomp. & $\%$ \\
\hline Age & $0.004^{\star \star}$ & -9.2 & $0.001^{\star \star}$ & -1.1 & -0.001 & 0.4 & 0.002 & -1.1 & $-0.006^{\star \star \star}$ & 4.4 \\
\hline Children ever born & $-0.008^{*}$ & 18.5 & -0.000 & 0.0 & -0.004 & 1.7 & -0.013 & 6.9 & $-0.013^{\star \star}$ & 9.6 \\
\hline No education & $-0.029^{* \star *}$ & 66.9 & $-0.019^{\star \star \star}$ & 18.3 & $-0.125^{\star \star \star}$ & 51.6 & $-0.070^{\star * *}$ & 36.9 & $-0.044^{\star \star *}$ & 32.6 \\
\hline 10 education & $0.013^{\star \star *}$ & -30.0 & $0.004^{*}$ & -3.5 & $0.011^{\star \star *}$ & -4.5 & $-0.001^{*}$ & 0.5 & $0.008^{\star \star}$ & -5.9 \\
\hline Rural & $-0.029^{* \star *}$ & 66.9 & $-0.033^{\star \star \star}$ & 32.2 & $-0.046^{\star \star *}$ & 19.0 & -0.003 & 1.6 & $-0.023^{\star \star \star}$ & 17.0 \\
\hline No religion & 0.000 & 0.0 & - & - & & & - & - & - & - \\
\hline Islam & -0.002 & 4.6 & - & - & $0.016^{\star \star \star}$ & -6.6 & 0.001 & -0.5 & 0.000 & 0.0 \\
\hline Traditional & 0.001 & -2.3 & - & - & 0.000 & 0.0 & -0.004 & 2.1 & - & - \\
\hline Animist & - & 0.0 & - & - & & & - & - & $-0.004^{\star \star \star}$ & 3.0 \\
\hline No partner & 0.000 & 0.0 & 0.000 & -0.1 & $0.001^{*}$ & -0.4 & 0.003 & -1.6 & 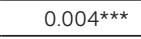 & -3.0 \\
\hline $\begin{array}{l}\text { Complication } \\
\text { information }\end{array}$ & 0.000 & 2.3 & $-0.033^{\star \star \star}$ & 32.3 & $-0.054^{\star \star \star}$ & 22.3 & -0.002 & 1.1 & $0.004^{\star \star *}$ & -3.0 \\
\hline Don't know & & & -0.000 & 0.1 & 0.000 & 0.0 & & & & \\
\hline Missing & & & -0.004 & 3.4 & $-0.018^{\star * *}$ & 7.4 & & & & \\
\hline $\begin{array}{l}\text { Health facility } \\
\text { permit }\end{array}$ & -0.001 & 16.1 & $-0.003^{\star \star}$ & 2.6 & -0.001 & 0.4 & 0.000 & 0.0 & - & - \\
\hline $\begin{array}{l}\text { Health facility } \\
\text { money }\end{array}$ & $-0.007^{\star \star \star}$ & 13.8 & -0.002 & 1.9 & $-0.003^{\star \star \star}$ & 1.2 & $-0.013^{\star \star \star}$ & 6.9 & - & - \\
\hline $\begin{array}{l}\text { Health facility } \\
\text { distance }\end{array}$ & $-0.006^{\star \star \star}$ & 0.0 & $-0.005^{\star \star \star}$ & 5.0 & -0.002 & 0.8 & -0.005 & 2.6 & - & - \\
\hline $\begin{array}{l}\text { Health facility } \\
\text { alone }\end{array}$ & 0.000 & 0.0 & $0.001^{\star \star \star}$ & -1.4 & 0.002 & -0.8 & 0.001 & -0.5 & - & - \\
\hline $\begin{array}{l}\text { Health facility } \\
\text { attitude }\end{array}$ & - & - & - & - & $0.006^{\star \star \star}$ & -2.5 & - & - & - & - \\
\hline Uninsured & - & - & - & - & & & $0.001^{*}$ & -0.5 & - & - \\
\hline Cascades & $-0.007^{* \star *}$ & 16.1 & - & - & & & - & - & - & - \\
\hline Centre & $-0.015^{\star \star \star}$ & 34.6 & - & - & & & - & - & - & - \\
\hline Centre Est & $-0.003^{* * *}$ & 6.9 & - & - & & & - & - & - & - \\
\hline Centre Nord & $0.001^{* *}$ & -2.3 & - & - & & & - & - & - & - \\
\hline Centre Ouest & 0.000 & 0.0 & - & - & & & - & - & - & - \\
\hline
\end{tabular}


Table 5.2 continued:

\begin{tabular}{|c|c|c|c|c|c|c|c|c|c|c|}
\hline \multicolumn{11}{|c|}{ Exposure to family planning information media source } \\
\hline & Burkina Faso & & Niger & & Nigeria & & Ghana & & enegal & \\
\hline Uninsured & - & - & - & - & & & $0.001^{*}$ & -0.5 & - & - \\
\hline Centre Sud & $-0.002^{\star \star \star}$ & 4.6 & - & - & & & - & - & - & - \\
\hline Est & $0.033^{\star \star *}$ & -76.1 & - & - & & & - & - & - & - \\
\hline Hauts Basins & $-0.013^{\star \star *}$ & 30.0 & - & - & & & - & - & - & - \\
\hline Nord & $-0.003^{\star \star \star}$ & 6.9 & - & - & & & - & - & - & - \\
\hline Plateau Central & $-0.009 * \star *$ & 20.8 & - & - & & & - & - & - & - \\
\hline Sahel & $0.025^{\star \star \star}$ & -57.7 & - & - & & & - & - & - & - \\
\hline Sud Ouest & $0.014^{\star \star \star}$ & -32.3 & - & - & & & - & - & - & - \\
\hline Agadez & - & - & -0.000 & 0.4 & & & - & - & - & - \\
\hline Diffa & - & - & $0.002^{\star \star \star}$ & -2.0 & & & - & - & - & - \\
\hline Dosso & - & - & $0.003^{*}$ & -2.9 & & & - & - & - & - \\
\hline Maradi & - & - & -0.002 & 1.7 & & & - & - & - & - \\
\hline Tahoua & - & - & -0.005 & 4.7 & & & - & - & - & - \\
\hline Tillaberi & - & - & $-0.007^{\star \star *}$ & 7.0 & & & - & - & - & - \\
\hline Zinder & - & - & -0.002 & 1.7 & & & - & - & - & - \\
\hline North Central & - & - & - & - & $0.016^{\star \star \star}$ & -6.6 & - & - & - & - \\
\hline North East & - & - & - & - & $-0.027^{* \star *}$ & 11.1 & - & - & - & - \\
\hline North West & - & - & - & - & $-0.043^{* \star *}$ & 17.7 & - & - & - & - \\
\hline South East & - & - & - & - & $0.014^{\star \star \star}$ & -5.8 & - & - & - & - \\
\hline South South & - & - & - & - & $0.016^{\star \star \star}$ & -6.6 & - & - & - & - \\
\hline Western & - & - & - & - & - & - & $-0.015^{\star \star \star}$ & 7.9 & - & - \\
\hline Central & - & - & - & - & - & - & $-0.017 * \star \star$ & 9.0 & - & - \\
\hline Volta & - & - & - & - & - & - & -0.001 & 0.5 & - & - \\
\hline Eastern & - & - & - & - & - & - & $0.003^{*}$ & -1.6 & - & - \\
\hline Greater Accra & - & - & - & - & - & - & $-0.019 * * \star$ & 10.0 & - & - \\
\hline Brong Ahafo & - & - & - & - & - & - & $-0.004^{* * *}$ & 2.1 & - & - \\
\hline Northern & - & - & - & - & - & - & -0.001 & 0.5 & - & - \\
\hline Upper East & - & - & - & - & - & - & $-0.034^{\star *}$ & 17.9 & - & - \\
\hline Upper West & - & - & - & - & - & - & 0.001 & -0.5 & - & - \\
\hline Ziguinchor & - & - & - & - & - & - & - & - & $0.005^{\star \star \star}$ & -3.7 \\
\hline Diourbel & - & - & - & - & - & - & - & - & $0.010^{\star \star \star}$ & -7.4 \\
\hline SaintLouis & - & - & - & - & - & - & - & - & $0.001^{*}$ & -0.7 \\
\hline Tambacounda & - & - & - & - & - & - & - & - & $-0.021^{\star \star \star}$ & 15.5 \\
\hline Kaolack & - & - & - & - & - & - & - & - & $-0.004^{\star \star \star}$ & 3.0 \\
\hline This & - & - & - & - & - & - & - & - & 0.000 & 0.0 \\
\hline Louga & - & - & - & - & - & - & - & - & $-0.008^{\star * *}$ & 5.9 \\
\hline Fatick & - & - & - & - & - & - & - & - & $-0.004^{\star \star *}$ & 3.0 \\
\hline Kolda & - & - & - & - & - & - & - & - & $-0.020 * \star \star *$ & 14.8 \\
\hline Matam & - & - & - & - & - & - & - & - & 0.000 & 0.0 \\
\hline Kaffrine & - & - & - & - & - & - & - & - & $-0.014^{\star \star}$ & 10.4 \\
\hline Kedougou & - & - & - & - & - & - & - & - & $-0.002^{\star \star \star}$ & 1.5 \\
\hline Sedhiou & - & - & - & - & - & - & - & - & $-0.004^{\star \star \star}$ & 3.0 \\
\hline $\mathrm{N}$ & 9831 & & 7645 & & 19911 & & 4147 & & 8839 & \\
\hline
\end{tabular}


INEQUALITIES IN REPRODUCTIVE HEALTH CARE USE IN FIVE WEST AFRICAN COUNTRIES: DECOMPOSITION ANALYSIS OF THE WEALTH-BASED GAPS IN BURKINA FASO, GHANA, NIGER, NIGERIA, SENEGAL

Table 5.3: Decomposition of modern contraceptive use

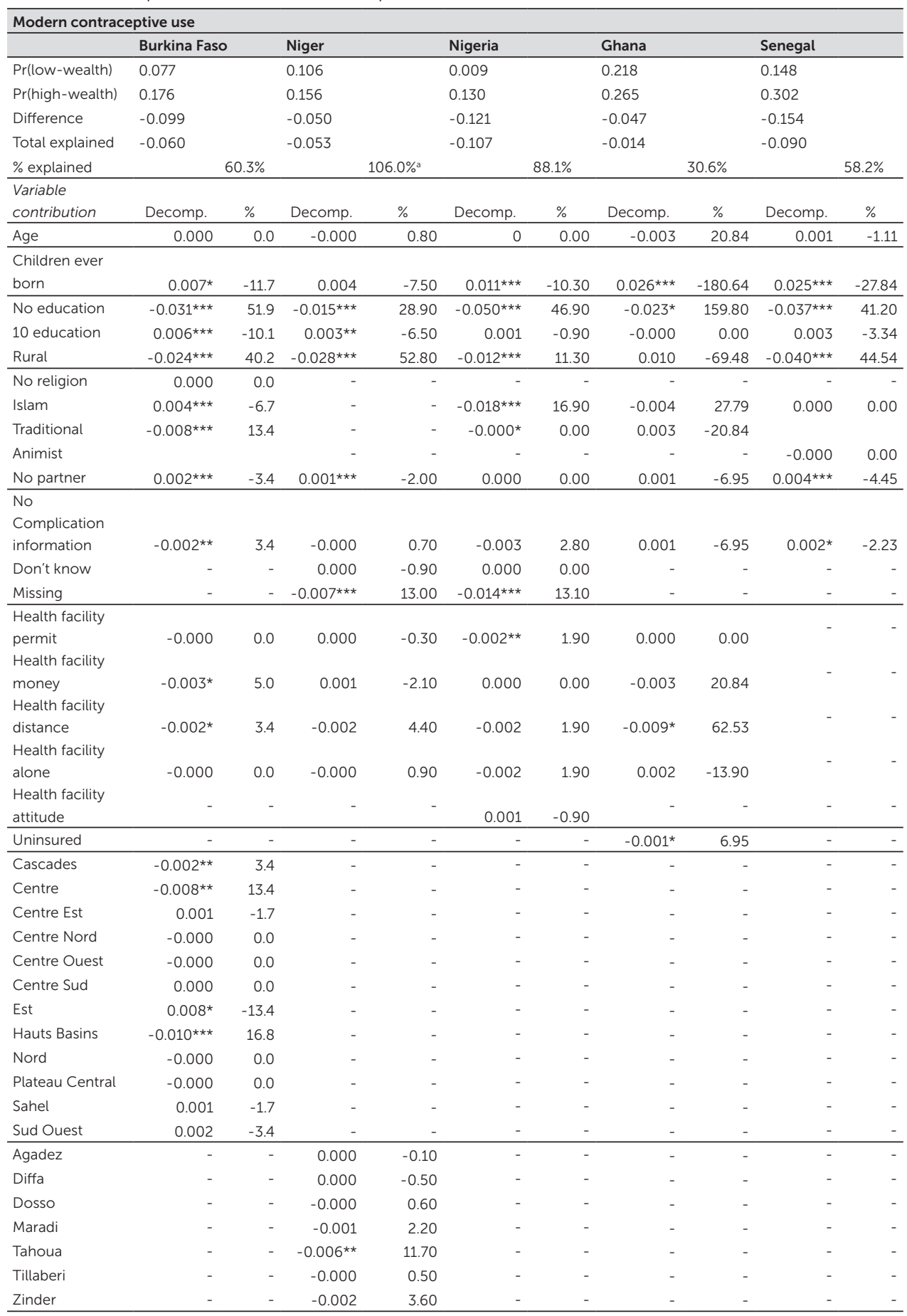


Table 5.3 continued:

\begin{tabular}{|c|c|c|c|c|c|c|c|c|c|c|}
\hline \multicolumn{11}{|c|}{ Modern contraceptive use } \\
\hline & Burkina Faso & & & & Nigeria & & Ghana & & Senegal & \\
\hline North Central & - & - & - & - & 0.000 & 0.00 & - & - & - & - \\
\hline North East & - & - & - & - & $-0.009^{* \star *}$ & 8.40 & - & - & - & - \\
\hline North West & - & - & - & - & $-0.016^{\star * *}$ & 15.00 & - & - & - & - \\
\hline South East & - & - & - & - & $0.004^{\star \star \star}$ & -3.80 & - & - & - & - \\
\hline South South & - & - & - & - & $0.004^{* * *}$ & -3.80 & - & - & - & - \\
\hline Western & - & - & - & - & - & - & -0.005 & 34.74 & - & 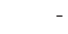 \\
\hline Central & - & - & - & - & - & - & $-0.012^{\star \star *}$ & 83.37 & - & - \\
\hline Volta & - & - & - & - & - & - & $0.001^{*}$ & -6.95 & - & - \\
\hline Eastern & - & - & - & - & - & - & -0.002 & 13.90 & - & - \\
\hline Greater Accra & - & - & - & - & - & - & -0.004 & 27.79 & - & - \\
\hline Brong Ahafo & - & - & - & - & - & - & $0.003^{* *}$ & -20.84 & - & - \\
\hline Northern & - & - & - & - & - & - & $-0.029 *$ & 201.48 & - & - \\
\hline Upper East & - & - & - & - & - & - & $0.027 *$ & -187.59 & - & - \\
\hline Upper West & - & - & - & - & - & - & 0.007 & -48.63 & - & - \\
\hline Ziguinchor & - & - & - & - & - & - & - & - & $0.002^{\star \star *}$ & -2.23 \\
\hline Diourbel & - & - & - & - & - & - & - & - & $0.010 * \star \star$ & -11.14 \\
\hline SaintLouis & - & - & - & - & - & - & - & - & 0.000 & 0.00 \\
\hline Tambacounda & - & - & - & - & - & - & - & - & $-0.014^{\star \star \star}$ & 15.59 \\
\hline Kaolack & - & - & - & - & - & - & - & - & $-0.005^{\star * *}$ & 5.57 \\
\hline This & - & - & - & - & - & - & - & - & 0.000 & 0.00 \\
\hline Louga & - & - & - & - & - & - & - & - & -0.001 & 1.11 \\
\hline Fatick & - & - & - & - & - & - & - & - & $-0.002^{*}$ & 2.23 \\
\hline Kolda & - & - & - & - & - & - & - & - & $-0.019 * \star \star$ & 21.16 \\
\hline Matam & - & - & - & - & - & - & - & - & 0.000 & 0.00 \\
\hline Kaffrine & - & - & - & - & - & - & - & - & $-0.013^{* \star *}$ & 14.48 \\
\hline Kedougou & - & - & - & - & - & - & - & - & $-0.001^{\star *}$ & 1.11 \\
\hline Sedhiou & - & - & - & - & - & - & - & - & $w 0.004^{\star \star \star *}$ & 4.45 \\
\hline $\mathrm{N}$ & 9838 & & 7654 & & 19959 & & 4147 & & 8839 & \\
\hline
\end{tabular}

* $p<0.05$ ** $p<0.01$ *** $p<0.001$

${ }^{1}$ The model explains $100 \%$ of the gap; the rest is noise due to the fact that the unexplained portion is negative. 
INEQUALITIES IN REPRODUCTIVE HEALTH CARE USE IN FIVE WEST AFRICAN COUNTRIES: DECOMPOSITION ANALYSIS OF THE WEALTH-BASED GAPS IN BURKINA FASO, GHANA, NIGER, NIGERIA, SENEGAL

Table 5.4: Decomposition of adequate antenatal visits

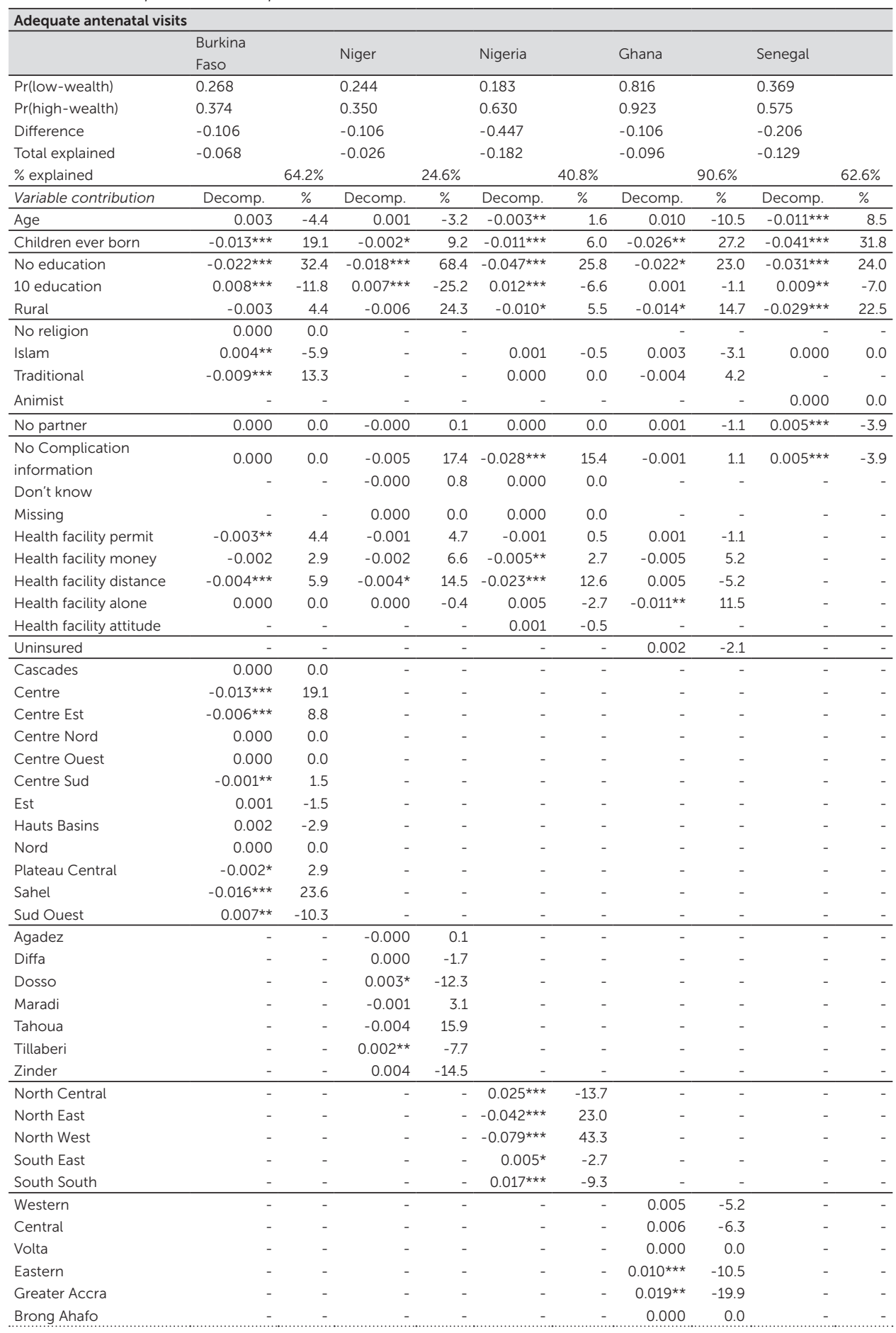


Table 5.4 continued:

\begin{tabular}{|c|c|c|c|c|c|c|c|c|c|c|}
\hline \multicolumn{11}{|c|}{ Adequate antenatal visits } \\
\hline & $\begin{array}{l}\text { Burkina } \\
\text { Faso }\end{array}$ & & & & & & Ghana & & Senegal & \\
\hline Upper East & - & - & - & - & - & - & 0.002 & -2.1 & - & - \\
\hline Upper West & - & - & - & - & - & - & -0.002 & 2.1 & - & - \\
\hline Ziguinchor & - & - & - & - & - & - & - & - & 0.000 & 0.0 \\
\hline SaintLouis & - & - & - & - & - & - & - & - & $0.001^{*}$ & -0.8 \\
\hline Tambacounda & - & - & - & - & - & - & - & - & 0.000 & 0.0 \\
\hline Kaolack & - & - & - & - & - & - & - & - & -0.001 & 0.8 \\
\hline This & - & - & - & - & - & - & - & - & 0.004 & -3.1 \\
\hline Louga & - & - & - & - & - & - & - & - & $-0.006^{\star * \star}$ & 4.7 \\
\hline Matam & - & - & - & - & - & - & - & - & $0.001^{\star *}$ & -0.8 \\
\hline Kaffrine & - & - & - & - & - & - & - & - & $-0.011^{*}$ & 8.5 \\
\hline Kedougou & - & - & - & - & - & - & - & - & -0.001 & 0.8 \\
\hline Sedhiou & - & - & - & - & - & - & - & - & $-0.003^{*}$ & 2.3 \\
\hline $\mathrm{N}$ & 9831 & & 7619 & & & & 4127 & & 8690 & \\
\hline
\end{tabular}

\section{Facility-based childbirth}

Table 5.5 shows that the wealth-based gap in the probability of having facility-based childbirth was 0.407 in Nigeria, 0.375 in Senegal, 0.320 in Ghana. The wealth gap was of smaller magnitude in Niger and Burkina Faso. Across the five countries, the characteristics included for each country model explained between $47 \%$ in Senegal and $90 \%$ in Nigeria of the observed wealth gap in facility-based childbirth. The wealth gap in facility-based childbirth also significantly increased by the number of children ever born by a woman across the countries and explains as much as $19 \%$ in Ghana and $17 \%$ in Senegal. The problem of needing permission to get medical help for self was significant in Burkina Faso and Niger, the problem of money to visit a health facility was significant only in Nigeria, and distance to the health facility was a significant problem in Burkina Faso, Niger, and Nigeria - all increasing the wealth gap. Regions, which most prominently contributed to the gap, were the Sahel in Burkina Faso, Zinder in Niger, North West region in Nigeria, Northern region in Ghana, and Kolda region in Senegal.

\section{C-section childbirth}

Table 5.6 shows that within the high-wealth group, women in Ghana had the highest probability of a C-section, 0.162 , followed by Senegal, 0.073 . Large wealth gaps in the probability of a $\mathrm{C}$-section between women groups were observed in Ghana and Senegal. Burkina Faso, Niger and Nigeria had a relatively lower probability of a C-section gap between wealth groups, however, the probability of a C-section was 
also relatively similar in these countries. Of the wealth-based difference in C-section, the characteristics included in the model explained $94.6 \%$ of the variation in Ghana, 79.1\% in Senegal, 94.1\% in Burkina Faso and 62.5\% in Niger. The characteristics included in the model explained the total low/high-wealth gap in C-section in Nigeria. The number of children ever born was statistically significant and increased the wealth gap in C-sections in Nigeria, Ghana, and Senegal. Significant health facility seeking characteristics that increased the wealth gap included money problems in Nigeria, Niger, and Ghana. Lack of pregnancy complication information increased the wealth gap in C-sections in Nigeria and Ghana. An increase in the wealth gap was significant only in the Sahel region in Burkina Faso and only the South-South region in Nigeria. No region in Niger contributed to the increased wealth gap but three regions were observed in Ghana and four in Senegal.

Table 5.5: Decomposition of facility-based childbirth

\begin{tabular}{|c|c|c|c|c|c|c|c|c|c|c|}
\hline \multicolumn{11}{|l|}{ Facility-based Childbirth } \\
\hline & $\begin{array}{l}\text { Burkina } \\
\text { Faso }\end{array}$ & & Niger & & Nigeria & & Ghana & & Senegal & \\
\hline Pr(low-wealth) & 0.581 & & 0.151 & & 0.063 & & 0.514 & & 0.499 & \\
\hline Pr(high-wealth) & 0.793 & & 0.382 & & 0.469 & & 0.833 & & 0.874 & \\
\hline Difference & -0.212 & & -0.232 & & -0.407 & & -0.32 & & -0.375 & \\
\hline Total explained & -0.142 & & -0.156 & & -0.369 & & -0.244 & & -0.179 & \\
\hline$\%$ explained & & $67.0 \%$ & & $67.24 \%$ & & $90.8 \%$ & & $76.3 \%$ & & $47.7 \%$ \\
\hline Variable contribution & Decomp. & $\%$ & Decomp. & $\%$ & Decomp. & $\%$ & Decomp. & $\%$ & Decomp. & $\%$ \\
\hline Age & 0.000 & 0.0 & 0.001 & -0.9 & $-0.006^{\star \star \star}$ & 1.6 & $0.007 * *$ & -2.9 & -0.001 & 0.6 \\
\hline Children ever born & $-0.008^{\star}$ & 5.6 & $-0.006^{\star \star \star}$ & 3.9 & $-0.015^{\star \star \star}$ & 4.1 & $-0.047^{\star \star *}$ & 19.2 & $-0.031^{\star * \star}$ & 17.3 \\
\hline No education & $-0.018^{\star \star \star}$ & 12.7 & $-0.022^{\star \star \star}$ & 14.4 & $-0.083^{\star \star \star}$ & 22.5 & $-0.053^{\star \star *}$ & 21.7 & $-0.029^{\star * \star}$ & 16.2 \\
\hline 10 education & 0.006 & -4.2 & 0.003 & -1.9 & $0.008^{* * *}$ & -2.2 & 0.002 & -0.8 & $0.008^{*}$ & -4.5 \\
\hline Rural & $-0.020^{\star \star \star}$ & 14.1 & $-0.069^{\star \star \star}$ & 44.3 & $-0.036^{\star \star \star}$ & 9.7 & $-0.068^{\star \star \star}$ & 27.8 & $-0.043^{\star \star \star}$ & 24.0 \\
\hline No religion & 0.000 & 0.0 & - & - & - & - & - & - & - & - \\
\hline Islam & $0.007^{\star \star \star}$ & -4.9 & - & - & $-0.026^{\star \star *}$ & 7.0 & 0.003 & -1.2 & 0.000 & 0.0 \\
\hline Traditional & $-0.016^{\star * \star}$ & 11.3 & - & - & 0.000 & 0.0 & -0.004 & 1.6 & - & - \\
\hline Animist & - & - & - & - & & & - & - & -0.001 & 0.6 \\
\hline No partner & 0.000 & 0.0 & -0.000 & 0.1 & $0.001^{* *}$ & -0.3 & 0.000 & 0.0 & 0.001 & -0.6 \\
\hline $\begin{array}{l}\text { No Complication } \\
\text { information }\end{array}$ & $-0.008^{\star \star \star}$ & 5.6 & $-0.013^{\star \star *}$ & 8.6 & $-0.024 * \star \star$ & 6.5 & $-0.003^{\star *}$ & 1.2 & $0.003^{\star * \star}$ & -1.7 \\
\hline Don't know & - & - & -0.000 & 0.1 & 0.000 & 0.0 & - & - & - & - \\
\hline Missing & - & - & $-0.025^{\star \star \star}$ & 15.7 & $-0.104^{\star * \star}$ & 28.2 & - & - & - & - \\
\hline Health facility permit & $-0.004^{\star \star \star}$ & 2.8 & $-0.002^{*}$ & 1.1 & -0.001 & 0.3 & -0.001 & 0.4 & - & - \\
\hline Health facility money & 0.000 & 0.0 & 0.001 & -0.3 & $-0.003^{\star \star *}$ & 0.8 & 0.006 & -2.5 & - & - \\
\hline Health facility distance & $-0.021^{\star \star \star}$ & 14.8 & $-0.013^{* * *}$ & 8.2 & $-0.008^{\star \star *}$ & 2.2 & -0.008 & 3.3 & - & - \\
\hline Health facility alone & 0.000 & 0.0 & -0.000 & 0.2 & -0.002 & 0.5 & -0.006 & 2.5 & - & - \\
\hline Health facility attitude & - & - & - & - & $0.003 * *$ & -0.8 & - & - & - & - \\
\hline Uninsured & - & - & - & - & - & - & $0.002^{*}$ & -0.8 & - & - \\
\hline Cascades & $-0.002^{\star}$ & 1.4 & - & - & - & - & - & - & - & - \\
\hline Centre & $-0.009 * * \star$ & 6.3 & - & - & - & - & - & - & - & - \\
\hline Centre Est & $-0.005^{\star \star \star}$ & 3.5 & - & - & - & - & - & - & - & - \\
\hline Centre Nord & 0.000 & 0.0 & - & - & - & - & - & - & - & - \\
\hline Centre Ouest & 0.000 & 0.0 & - & - & - & - & - & - & - & - \\
\hline Centre Sud & $-0.003 * \star *$ & 2.1 & - & - & - & - & - & - & - & - \\
\hline Est & $-0.008^{\star *}$ & 5.6 & - & - & - & - & - & - & - & - \\
\hline Hauts Basins & 0.000 & 0.0 & - & - & - & - & - & - & - & - \\
\hline Nord & 0.001 & -0.7 & - & - & - & - & - & - & - & - \\
\hline
\end{tabular}


Table 5.5 continued:

\begin{tabular}{|c|c|c|c|c|c|c|c|c|c|c|}
\hline \multicolumn{11}{|c|}{ Facility-based Childbirth } \\
\hline & $\begin{array}{l}\text { Burkina } \\
\text { Faso }\end{array}$ & & iger & & Nigeria & & Ghana & & Senegal & \\
\hline Plateau Central & $-0.004^{\star \star \star}$ & 2.8 & - & - & - & - & - & - & - & - \\
\hline Sahel & $-0.024^{\star \star \star}$ & 16.9 & - & - & - & - & - & - & - & - \\
\hline Sud Ouest & $-0.005^{*}$ & 3.5 & - & - & - & - & - & - & - & - \\
\hline Agadez & - & - & 0.000 & -0.1 & - & - & - & - & - & - \\
\hline Diffa & - & - & -0.001 & 0.4 & - & - & - & - & - & - \\
\hline Dosso & - & - & 0.001 & -0.8 & - & - & - & - & - & - \\
\hline Maradi & - & - & -0.001 & 0.7 & - & - & - & - & - & - \\
\hline Tahoua & - & - & -0.005 & 3.1 & - & - & - & - & - & - \\
\hline Tillaberi & - & - & 0.000 & -0.2 & - & - & - & - & - & - \\
\hline Zinder & - & - & $-0.005^{\star}$ & 3.5 & - & - & - & - & - & - \\
\hline North Central & - & - & - & - & 0.002 & -0.5 & - & - & - & - \\
\hline North East & - & - & - & - & $-0.029 * * *$ & 7.8 & - & - & - & - \\
\hline North West & - & - & - & - & $-0.056^{\star * *}$ & 15.2 & - & - & - & - \\
\hline South East & - & - & - & - & $-0.005^{\star \star \star}$ & 1.4 & - & - & - & - \\
\hline South South & - & - & - & - & $0.013^{\star \star \star}$ & -3.5 & - & - & - & - \\
\hline Western & - & - & - & - & - & - & $0.011^{\star \star \star}$ & -4.5 & - & - \\
\hline Central & - & - & - & - & - & - & $0.018^{\star * \star}$ & -7.4 & - & - \\
\hline Volta & - & - & - & - & - & - & 0.000 & 0.0 & - & - \\
\hline Eastern & - & - & - & - & - & - & $0.005^{\star \star *}$ & -2.0 & - & - \\
\hline Greater Accra & - & - & - & - & - & - & 0.003 & -1.2 & - & - \\
\hline Brong Ahafo & - & - & - & - & - & - & 0.000 & 0.0 & - & - \\
\hline Northern & - & - & - & - & - & - & $-0.117^{\star \star \star *}$ & 47.9 & - & - \\
\hline Upper East & - & - & - & - & - & - & 0.006 & -2.5 & - & - \\
\hline Upper West & - & - & - & - & - & - & 0.000 & 0.0 & - & - \\
\hline Ziguinchor & - & - & - & - & - & - & - & - & 0.001 & -0.6 \\
\hline Diourbel & - & - & - & - & - & - & - & - & 0.000 & 0.0 \\
\hline SaintLouis & - & - & - & - & - & - & - & - & 0.000 & 0.0 \\
\hline Tambacounda & - & - & - & - & - & - & - & - & $-0.023^{\star * \star}$ & 12.8 \\
\hline Kaolack & - & - & - & - & - & - & - & - & 0.000 & 0.0 \\
\hline This & - & - & - & - & - & - & - & - & $-0.007^{\star *}$ & 3.9 \\
\hline Louga & - & - & - & - & - & - & - & - & 0.000 & 0.0 \\
\hline Fatick & - & - & - & - & - & - & - & - & 0.000 & 0.0 \\
\hline Kolda & - & - & - & - & - & - & - & - & $-0.038^{\star \star \star}$ & 21.2 \\
\hline Matam & - & - & - & - & - & - & - & - & $0.002^{*}$ & -1.1 \\
\hline Kaffrine & - & - & - & - & - & - & - & - & $-0.012^{\star *}$ & 6.7 \\
\hline Kedougou & - & - & - & - & - & - & - & - & $-0.002^{\star \star *}$ & 1.1 \\
\hline Sedhiou & - & - & - & - & - & - & - & - & $-0.005^{\star \star *}$ & 2.8 \\
\hline $\mathrm{N}$ & 9832 & & 7652 & & 19919 & & 4146 & & 8839 & \\
\hline
\end{tabular}


INEQUALITIES IN REPRODUCTIVE HEALTH CARE USE IN FIVE WEST AFRICAN COUNTRIES: DECOMPOSITION ANALYSIS OF THE WEALTH-BASED GAPS IN BURKINA FASO, GHANA, NIGER, NIGERIA, SENEGAL

Table 5.6: Decomposition of C-Section

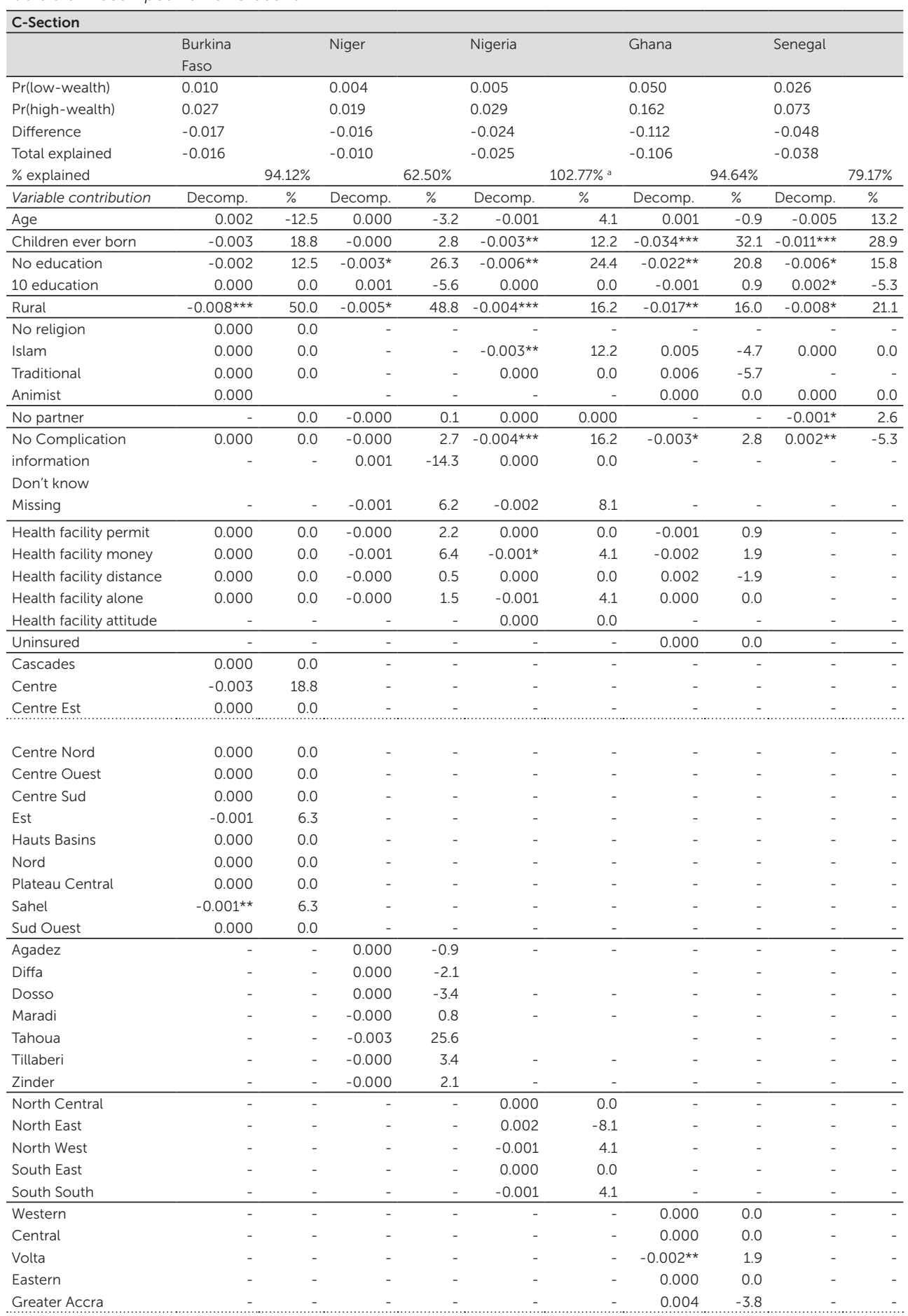


Table 5.6 continued:

\begin{tabular}{|c|c|c|c|c|c|c|c|c|c|c|}
\hline \multicolumn{11}{|l|}{ C-Section } \\
\hline & $\begin{array}{l}\text { Burkina } \\
\text { Faso }\end{array}$ & \multicolumn{2}{|c|}{ Niger } & \multicolumn{2}{|c|}{ Nigeria } & \multicolumn{2}{|r|}{ Ghana } & \multicolumn{3}{|c|}{ Senegal } \\
\hline Brong Ahafo & - & - & - & - & - & - & $-0.002^{\star}$ & 1.9 & - & - \\
\hline Northern & - & - & - & - & - & - & $-0.027 * \star *$ & 25.5 & - & - \\
\hline Upper East & - & - & - & - & - & - & -0.012 & 11.3 & - & - \\
\hline Upper West & - & - & - & - & - & - & -0.003 & 2.8 & - & - \\
\hline Ziguinchor & - & - & - & - & - & - & - & - & 0.001 & -2.6 \\
\hline Diourbel & - & - & - & - & - & - & - & - & -0.001 & 2.6 \\
\hline SaintLouis & - & - & - & - & - & - & - & - & 0.000 & 0.0 \\
\hline Tambacounda & - & - & - & - & - & - & - & - & -0.002 & 5.3 \\
\hline Kaolack & - & - & - & - & - & - & - & - & $-0.001^{\star}$ & 2.6 \\
\hline This & - & - & - & - & - & - & - & - & $0.002^{*}$ & -5.3 \\
\hline Louga & - & - & - & - & - & - & - & - & 0.000 & 0.0 \\
\hline Fatick & - & - & - & - & - & - & - & - & $-0.001^{*}$ & 2.6 \\
\hline Kolda & - & - & - & - & - & - & - & - & $-0.005^{\star}$ & 13.2 \\
\hline Matam & - & - & - & - & - & - & - & - & 0.000 & 0.0 \\
\hline Kaffrine & - & - & - & - & - & - & - & - & -0.003 & 7.9 \\
\hline Kedougou & - & - & - & - & - & - & - & - & 0.000 & 0.0 \\
\hline Sedhiou & - & - & - & - & - & - & - & - & $-0.001^{*}$ & 2.6 \\
\hline $\mathrm{N}$ & 9838 & & 7654 & & & & 4147 & & 8750 & \\
\hline
\end{tabular}

* $p<0.05 * * p<0.01 * * * p<0.001$

a The model explains $100 \%$ of the gap; the rest is noise due to the fact that the unexplained portion is negative.

\subsection{DISCUSSION}

Our findings confirm the presence of wealth-based inequalities to the detriment of women in low-wealth households in the use of reproductive health care services. These results are in line with other studies, which have established the disadvantage of poor women in the use of reproductive health care services in sub-Saharan Africa (Alam et al., 2015; Gakidou \& Vayena, 2007; Hosseinpoor et al., 2011). The findings show that differences in characteristics between the two wealth groups explain a considerable part of the wealth gap in all five reproductive health care services we studied. More importantly, differences in the distribution of observed maternal characteristics contribute to the observed wealth-based inequalities in reproductive health care use in all five countries. There is evidence to suggest that the probability of the use of reproductive health care services is not parallel to the inequality gap. We find that the probability of women in low-wealth households to use reproductive health care services is higher in Burkina Faso for exposure to family planning information via mass media and facility-based childbirth services, while it is higher in Ghana for modern contraceptives, an adequate number of antenatal visits and C-Sections. However, we observe that the wealth-based inequalities in reproductive health care use are higher in other countries; in Nigeria for exposure to family planning information via mass media, an adequate number of antenatal visits and facility-based childbirth, while Senegal has 
a higher inequality gap in modern contraceptive use and C-section. This observation could be due to coverage of the cost of services by third parties (Countdown Equity Analysis et al., 2008).

We observe that $71 \%$ of the disparity in exposure to family planning information via mass media is explained by sociodemographic differences between women in the different wealth groups. This suggests that poorer women have characteristics that prevent them from receiving family planning messages. Sociodemographic characteristics such as religion, residence, and marital status negatively influence the exposure to family planning information, which has also been observed in other studies (Ajaero et al., 2016; Babalola, Folda, \& Babayaro, 2008). Religion could constrain the use of reproductive health care services. Theological differences, norms and characteristics of religious groups may explain this observation (Gyimah et al., 2006). Place of residence determines how accessible and available family planning information is. It is reported that urban areas have easier accessible family planning services (Hounton et al., 2015). Regarding marital status, in some communities, married women are less autonomous. Thus, their restricted use of family planning services has been explained through having to conform to social norms (Ngome \& Odimegwu, 2014). It can, therefore, be said that family planning information via mass media has not been well targeted to the poor population group, and does not address their peculiarities. For example, some religious practices encourage polygamy and do not encourage family planning programs (Ajaero et al., 2016; Guengant, 2012; Onwuzurike \& Uzochukwu, 2001). Moreover, policies to disseminate information via mass media by governments, seldom address differentials in the characteristics of women or remain unimplemented. One such policy is Nigeria's national reproductive health policy and strategy to promote reproductive health education through mass media (Ahonsi, 2015; $\mathrm{FMoH}, 2001)$. Alternatively, the urban reproductive health initiative in Senegal takes into consideration the influence of religion and attempts to fill the gaps in exposure to family planning information across urban dwellers (Benson et al., 2018; Krenn, Cobb, Babalola, Odeku, \& Kusemiju, 2014). Population-wide policies are too broad and often benefit the better-off. Interventions, which do not promote fairness in access for most vulnerable groups, usually widen the inequality gaps.

Unsurprisingly, differences in maternal characteristics increase inequality and explain the observed gap in modern contraceptive use between the poorest and wealthier women. Though the average modern contraceptive use is appreciably low (Figure 5.1), country policies have singled-out what is included in reproductive health care packages. In Ghana, for example, the NHIS which covers maternal care does not cover family 
planning services (Witter et al., 2007). We observe a significant contribution of rural disparity in other countries but not in Ghana. Perhaps the community-based health planning and services are better able to address the problem of service availability in remote areas and rural districts of Ghana. There however seems to be only a providerfocused delivery strategy to increase the prevalence of modern contraceptive prevalence and so ignoring individual factors, such as distance, which explains as much as $62 \%$ of the disparity in the use of modern contraceptives in Ghana. The distance to a health facility is a problem because it is related to the indirect costs of transportation, which are incurred when seeking modern contraceptives while these costs are not covered under any national health promotion scheme and thus, they constitute a burden for poor women. The association of distance and contraceptive use echoes earlier studies that indicate a contraceptive use decline among women, who are five kilometers away from a health facility in Burkina Faso (Wulifan et al., 2017) and two kilometers away from a community-based health planning centers in the Upper East region of Ghana (Achana et al., 2015). Further, the trend across regions suggests that in addition to the imbalanced distribution of resources, the region is relevant to understand the disparities in family planning services. Further research is needed to understand the sociocultural factors that cause regional disparities. Health inequalities are a result of a variety of demand-side as well as supply-side factors (Witter et al., 2016). Such evidence could be valuable input for developing a policy to encourage not only the use of family planning commodities but all aspects of reproductive health care.

Moreover, our three indicators of maternal health utilization suggest that not all countries, which have abolished user fees for maternal care completely, have performed similarly in reducing the inequality between wealth groups. Nonetheless, it appears that in countries with complete fee exemption policies, that is, Ghana, Niger, and Senegal, the between wealth groups gaps in having an adequate number of ANC visits, facility-based childbirth and C-section are smaller. Although, comparatively, it seems that Senegal has not achieved as much as the other two countries which can allude to the fact that unlike Ghana and Niger, Senegal's maternal care policy does not cover ANC (Witter et al., 2010). From our findings, it appears that the poorest women remain unable to surmount other barriers that prevent them from using subsidized facility-based childbirth care. We find that the number of children ever born by a woman, the cost of transportation and cost at the point of use are significant barriers that deter women from the appropriate use of ANC services. These are also consistent with findings from previous studies (De Allegri et al., 2011; Do et al., 2015; Koster et al., 2016; Mwase et al., 2018). This is an indication that the abolishment of the user fee for maternal care services may contribute to wealthbased inequality in the use of these services. 
The explanatory variables included for Nigeria explain more than $100 \%$ of the gap, indicating that the covariates explained more than the observed difference in rates of use. Thus, the unexplained gap in C-section between the poorest and wealthier women contributes positively to the gap. This finding suggests that the covariates included for poor women explain all the observed disparity in having a C-section. It is also likely that Nigeria's removal of user fees policy for C-section through the national health insurance scheme, maternal and child health project, has fostered more equitable access to obstetric health care services especially among women of lower economic status (Mohammed \& Dong, 2012). The overall coverage of the project, however, is low and fragmented (Onwujekwe et al., 2016). Elimination of user fees for maternal care services like C-sections goes only a short way in alleviating the out-ofpocket costs incurred when seeking care and increase the wealth-based inequalities to the detriment of poorer households. Evidence of this is the wealth gap observed in C-section between women in low-wealth and high wealth households in Ghana. Chapters 3 and 4 showed that poorer women remain at a disadvantage of Ghana's fee exemption policy for childbirth care despite the policy. In other countries with policies that also cover $\mathrm{C}$-section, gaps in reproductive health care use between the wealth groups persist. For example, a user fee policy in Burkina Faso exempts the poorest women from fees in the case of $\mathrm{C}$-section childbirth. There is also the free $\mathrm{C}$-section policy in Senegal. Comparatively, the inequality gap in C-section is lower in Burkina Faso than Senegal. This suggests that aside service fees, other barriers encountered by the poorest to use the subsidized services remain insurmountable. This finding is corroborated by other studies (Cisse, Faye, de Bernis, Dujardin, \& Diadhiou, 1998; Richard et al., 2013). A study in Senegal shows that the physical distribution of facilities with C-section capabilities does not favor the grassroots women (Witter et al., 2010). A study in Mali discussed that while richer households can raise emergency funds needed to access care services, the poorest households have much more difficulty doing so (Arsenault et al., 2013).

Our study indicates that other factors, aside the cost of services at the point of use, contributed to the inequality in the use of subsidized maternal health care. Cost of transport, as well as money spent on services at the health facility, are not always fully reimbursed under health schemes. A subpopulation study in Nigeria describing the free maternal health care intervention effect, observes that the use of antenatal health care reduces with distance despite free maternal care policy (Edu et al., 2017). Other studies clarify that money for health facility hinders women from profiting from the national maternal health care subsidy policy (Mwase et al., 2018). Another maternal characteristic, namely knowledge of pregnancy care, added to the disadvantage of 
poor women. The lack of information on pregnancy complications among poor women increases the gap in the use of reproductive health care, including adequate ANC visits. Information dissemination about the free maternal health childbirth is ineffective and disadvantaged women in poor households are unable to take benefit (Edu et al., 2017). The problem of needing permission to get medical help for self adds to the disadvantage of the poorest households, which they have when needing reproductive health care services, including modern contraceptives and antenatal. Eliminating user fees could have impacted on women's empowerment and their ability to get to a health facility. Studies have confirmed the existence of settings where women require spousal or family permission or escort to make use of reproductive care (Some, Sombie, \& Meda, 2013). The number of children ever born by a woman in poor households increased the wealth-based gap. In addition to economic challenges when seeking care, women who have gone through childbirth would rely on previous experiences such as the negative attitude of health workers and travel difficulties when deciding on the use of reproductive health care (Arthur, 2012; Pell et al., 2013). Regional disparities observed within all countries implicate cultural differences or structural inadequacies in the availability and distribution of reproductive health care. Findings from other studies confirm that unfair allocation of resources contribute to geographical disparity, concluding that supply-side policies addressed at wealth inequalities in utilization of care are ineffective if they do not account for social determinants of health (Liu et al., 2014; Onarheim et al., 2015; Skaftun, Ali, \& Norheim, 2014).

For policy purposes, it is necessary to explore a practical and sustainable way to address wealth-based inequality in reproductive health care use. Governments in resourcepoor West African countries, need to design reproductive health care programs, which target additional services for the poor. The strategies towards universal health coverage should capture not just everybody being covered but should pay attention to the allocation of health services to groups with the highest needs - typically poor groups. This will maximize the intervention impact and cost-effectiveness. Based on our findings, a policy targeting sociodemographic determinants of health to capture the differences in marital status, financial expenditure for accessing health services, knowledge of pregnancy care, and geographical location, will be preferred.

\section{Study limitation}

This study has some limitations that need to be acknowledged. Many characteristics included in the study, are likely to be related to the use of reproductive health care but there are also other factors likely to be related to use, for example, health condition during pregnancy, which we could not control for. In addition, there are important 
observable and non-observable differences between service users and non-users which differ from a country to another. These factors could not be included given data constraints. We acknowledge that neither the poor nor the rich can use services that do not exist while our study does not analyze coverage gaps. We analyzed five countries in West Africa with surveys conducted in different years. Although this does not take away from the results or the recommendations, comparability must be done with caution. This chapter does not address questions on the causes of poverty but rather touches on inequalities in the troublesome issues of social determinants of health. Lastly, cross-sectional survey data can only reveal association not necessarily a causal relationship between health outcomes and covariates.

\subsection{CONCLUSION}

Using national-level data from Burkina Faso, Niger, Nigeria, Ghana and Senegal, the results of this study elucidate that women in households with low wealth are at a disadvantage despite reproductive health care policies to eliminate inequalities. Inequalities exist due to differences in the characteristics between the low wealth and wealthier groups of women. Evidence of this is in the substantial proportions of the explained gap by maternal characteristics. All countries analyzed have or are in the process of nationwide interventions to improve maternal health care. In all five countries, women of reproductive ages in the poorest households have lower chances of getting reproductive health care services. Although the contribution of these characteristics differs among countries, they remain relevant barriers to the use of reproductive health care services. With the introduction of targeted policies to mitigate the impact of these contributors, vulnerable women's use of reproductive health care services could be increased at least to the level of their better-off counterparts in wealthier households. Such as engaging companions and provision of transportation to reproductive health care centers to mitigate the impact of permission problems when in need of medical help. Furthermore, communication strategies and the provision of family planning services that specifically target women in poor households need to be developed. These should be mindful of fertility preference, religion, residence, and marital status to increase access to family planning services. It is also important to expand family planning efforts to include reimbursement or fee exemption policies similar to maternal health care schemes to improve childbirth services for both aspects of reproductive health care. 

GENERAL DISCUSSION 


\subsection{INTRODUCTION}

The principal aim of this dissertation was to increase our knowledge and understanding of the determinants of inequalities in reproductive health care among women in SubSaharan Africa. The case study countries, Ghana and Nigeria, like other Sub-Saharan African countries, have a steep wealth-gradient in the use of reproductive health care services (GSS et al., 2015; NPC \& ICF, 2014). Although there are policies and interventions in Ghana and Nigeria that address these reproductive health care issues, evidence shows that improvements in service use have not been fairly spread across all social groups (GSS, GHS, \& ICF, 2009; GSS et al., 2015; NPC, 2009; NPC \& ICF, 2014). To improve the reproductive health of women of childbearing age, and to address the stalling maternal mortality across most of Sub-Saharan Africa, it is expedient that interventions focus on the barriers to the use of reproductive health care services, particularly, those that inhibit the disadvantaged populations from benefitting fully from on-going interventions. Poor women, for example, have higher fertility rates but low service use rates (De Brouwere, Richard, \& Witter, 2010; WHO, 2015b). For this purpose, the extent and determinants of inequalities in reproductive health care in Sub-Saharan Africa need to be identified. As mentioned in Chapter 1, revealing group inequalities in reproductive health care use provides scientific evidence for policy formulation. However, such evidence is still lacking. To comprehend the disparities in the use of reproductive health care services among women, the factors that characterize disadvantaged groups need to be assessed.

Four research questions were formulated in Chapter 1 of this dissertation and each of the subsequent core chapters focused on one of these questions. In particular, Chapter 2 determines the variations in the magnitudes of socioeconomic inequalities across reproductive health care services and countries in Sub-Saharan Africa. The analysis is based on a systematic review and meta-analysis of the academic literature on socioeconomic inequalities in reproductive care in Sub-Saharan Africa, published in the last decade. The review elucidates the recent evidence on improvements in or lack of equitable use of services across the region. In this chapter, we find that the socioeconomic inequality in the use of essential reproductive health care services is pro-wealthy (i.e. wealthier women use more services), especially skilled childbirth, throughout Sub-Saharan African countries. However, countries with lower average inequality in the use of reproductive health care services, do not necessarily have a higher coverage of these services.

Next, in Chapter 3 of this dissertation, we investigated the patterns of reproductive 
health care use by women in Ghana and Nigeria. The analysis is based on the method of cluster analysis. In addition, we use multinomial logistic regression to identify socioeconomic factors associated with cluster membership. The analysis produces context-specific evidence on the differences in reproductive health care coverage among women in the two countries. It also estimates the chances of using reproductive health care services given the individual sociodemographic and economic characteristics. We find that women with peculiar characteristics (e.g. uneducated, services and manual occupation, sales, low wealth households, or lack insurance) are likely to use less quality family planning and maternal health care services in Ghana and Nigeria.

In Chapters 4 and 5, we then investigated if policies to reduce the gaps in the use of reproductive health care services between groups, benefit all women irrespective of socioeconomic position. Using cross-sectional country-level data from Ghana and Nigeria, in Chapter 4, concentration curves and concentration indices are used to plot and quantify socioeconomic-related inequalities in the use of reproductive health care services over a period of 20 years. By examining the changes in the inequalities in reproductive health care use between socioeconomic groups over time, we provide insights into horizontal inequalities and coverage gaps. We find declining inequalities on some indicators of family planning and maternal health care services. Essential reproductive health care services, however, remain advantageous for wealthier women. The comparison across socioeconomic groups shows a varying magnitude of inequality in the use of reproductive health care services within and between the groups. That is age, marital status, maternal occupation, and geographical residence groups. In Chapter 5, we further examine the contribution of individual sociodemographic characteristics in Ghana and Nigeria to observed inequalities in reproductive health care service use, in comparison to other countries in the region. Data from the countrywide surveys from the DHS of Burkina Faso, Niger, Nigeria, Ghana, and Senegal are analyzed. The method of decomposition analysis is used to quantify the contribution of various sociodemographic characteristics to wealthbased disparities in the use of reproductive health care services. The results confirm that differences in characteristics between the poorest and wealthiest women explain the substantial disparity observable in the use of reproductive health care services across the five West African countries.

In this final discussion chapter of the dissertation, Chapter 6, we present and discuss the main findings of the dissertation in the form of 5 key statements. 


\title{
6.2. DISCUSSION OF STATEMENTS BASED ON THE MAIN FINDINGS
}

\author{
Statement 1: The emphasis on childbirth services across Sub-Saharan African \\ countries pulls attention away from pre-pregnancy, pregnancy, emergency \\ obstetrics, and postpartum services.
}

The literature review in Chapter 2 indicates that across the countries in Sub-Saharan Africa, skilled childbirth services have the largest wealth-based inequalities in service use compared to other reproductive health care services. Other services, prepregnancy, pregnancy, emergency obstetrics, and postpartum services have less prowealthy inequality. Nevertheless, skilled childbirth services have been high on policy agendas in Sub-Saharan Africa due to initiatives that prioritize the reduction in maternal deaths. The WHO started monitoring maternal mortality in 1983. Subsequently, in 1985, a seminal paper "Maternal mortality-a neglected tragedy" called for prioritization of maternal mortality, alluding to the estimated 500,000 annual maternal deaths in developing countries (Rosenfield \& Maine, 1985). Since then, global attention to maternal mortality has been highlighted in several conferences including the 1994 International Conference on Population and Development in Cairo, Egypt, which resulted in the key steering document "World Population Plan of Action". Reducing national and sub-national maternal mortality levels stood out as the main target of this conference and other summits in the last three decades. Although the maternal mortality rates in Sub-Saharan Africa have been reduced, they are still considerably high compared to the rest of the world.

Therefore, as also indicated by the results in this dissertation, there is a need for policies that focus on further improving access to skilled childbirth services as well as all other reproductive health care services. Previous research has suggested that low-income countries can mitigate inequity in service use through subsidized service or exemptions from user fees (Witter, 2009). Therefore, in many Sub-Saharan African countries, skilled childbirth services are subsidized or users may qualify for fee exemptions for facility-based births (De Allegri et al., 2012; Hatt et al., 2013; Witter et al., 2010). This is however not always the case for other essential pre-pregnancy, pregnancy, emergency obstetrics, and postpartum services along the continuum of care for the reproductive health of women (Chapter 2). Progress in the coverage of supply-side interventions, moving from the current focus on childbirth services to a focus on all reproductive health care services, is important to fully address women's needs (Kerber et al., 2007). Supply-side improvements, such as better quality and accessibility, can have positive effects on the utilization of reproductive health care services and can 
help to address equity issues (Mangham-Jefferies, Pitt, Cousens, Mills, \& Schellenberg, 2014). The findings in this dissertation confirm that in Sub-Saharan African countries, the utilization of skilled childbirth services is higher in case of user fee exemption for facility-based childbirth. In other words, women use services more when available and accessible. The same applies to other reproductive health care services. In countries with user fee exemption for reproductive health care, the proportion of women using reproductive health care services is higher when compared with countries without an exemption policy (Chapter 2 and 5). However, for women with low socioeconomic status, who have disproportionately lower use of all reproductive health care services due to transportation and other indirect costs, unskilled reproductive care providers offer an alternative to obtaining care (Chapter 2 and 3). Thus, through the increased provision of good quality reproductive health care, service use among the poor and low socioeconomic class can be addressed. Research has shown a relationship between the increase in the supply of health care services and positive effects on health care seeking behavior (Fichera, Gray, \& Sutton, 2016; Smith \& Sulzbach, 2008). It is essential to expand the user fee exemption policies across Sub-Saharan Africa to cover all related reproductive health care services and all women's groups. This will help to address the wealth-related differences in service availability and provision. This has the potential to spur demand for services among users.

Reproductive health care policies are important in improving the wellbeing of women. For women in their reproductive years, all aspects of reproductive health care services are as important as facility-based childbirth attended by a skilled professional. For society, morbidity is as important as mortality. Conversely, for the prevention of mortality, user fee exemption has been the favored approach among policymakers in most Sub-Saharan Africa countries (De Brouwere et al., 2010; Ridde \& Morestin, 2011). This has been intensified to attain MDG 5 targeting a three-quarters reduction in maternal mortality by 2015 (WHO, 2005a). As explained in Chapter 2, most reproductive health care programs have paid attention to non-user factors of childbirth care by focusing on the provision of skilled birth attendants primarily at health facilities (Koblinsky et al., 2006; Lassi et al., 2014; Moller et al.). Furthermore, the shift in focus from attempting to predict and prevent complications and deaths among women during pregnancy to an increase in access to emergency obstetric care has resulted in other needed services for women to be overlooked. Before or during pregnancy, anemia, malaria, HIV/AIDS, heart, lung, liver, or kidneys diseases, and ectopic pregnancies increase the risk of maternal deaths. These account for up to $25 \%$ of maternal deaths (WHO, 2005b). Unsafe abortion accounts for up to about 50\% of maternal deaths in SubSaharan Africa (Rogo, Oucho, \& Mwalali, 2006). Research among maternal health 
care providers indicates that knowledge, practice, and provision of safe motherhood are still inadequate (ljadunola et al., 2010; Jonas, Crutzen, van den Borne, \& Reddy, 2017; Ministry of Health Ghana, 2008). Health worker training on safe motherhood is noted to fade away from Ghana's maternal health policy and programs (Koduah et al., 2016). In turn, the components of safe motherhood that would provide women with information on pregnancy complications and readiness, postpartum family planning, and birth preparedness among others, are rarely delivered to clients. This contributes to the low demand for these services.

We conclude that initiatives to decrease maternal mortality through higher use of skilled birth attendants in Sub-Saharan African are vital but have had the undesirable effect of decreased coverage of non-childbirth related services. Furthermore, availability and provision of quality reproductive health care services are limited and contribute to the low demand and use of other care services aside from facility-based childbirth with a skilled birth attendant (Gage, Ilombu, \& Akinyemi, 2016; Ijadunola et al., 2010; Jonas et al., 2017). Evidence shows that the neglect of services in the continuum of reproductive health care services, occurs in pre-conception, prenatal, and postnatal health care. This suggests that reproductive health care policies in SubSaharan African countries are uncomprehensive in terms of the proportion of services covered. Prioritization of all aspects of reproductive health care services needs to be the guiding principle in new policy initiatives.

\section{Research and policy implications}

As suggested by the above discussion, the design and implementation of reproductive health care policies should focus on addressing the needs of fecund women before, during and after pregnancy. The lack of comprehensive and adequately formulated policies may lead to the neglect of pre-pregnancy, pregnancy, emergency obstetrics, and postpartum services. Notably, across countries, in particular countries with subsidies or exemption policies, reproductive health care services are used more frequently. However, such policies, if not applied across the continuum of reproductive health care, ignore reproductive health care services not covered by these policies. This also suggests that demand for services can be generated especially among disadvantaged women sub-groups if all essential reproductive health care services are subsidized or exempted from user fees.

Future studies should investigate the priority setting processes in reproductive health care, in particular, the processes of decision-making and how policy actors define and prioritize inequities. The recognition of context-specific health care priorities 
through research could further inform the design, implementation, and content of reproductive health care initiatives. Also, qualitative research is needed to determine effective context-specific means to deliver reproductive health care services to reach disadvantaged populations. In a subsequent study, one-on-one and focus group interviews among women of reproductive ages can provide in-depth knowledge on the socio-cultural factors while a similar approach can document contextappropriate socio-political circumstances among policymakers and physicians gatekeepers. These methods will ensure that appropriate contexts within a country are captured to design interventions that will be acceptable for communities. In so doing, a subsequent study could contextualize the findings into the socio-cultural and sociopolitical situation in the two countries, and reflect on the differences between the two institutional contexts.

To address the continuum of reproductive health care, it is necessary to expand the coverage of services. It is, however, important to consider contextual characteristics of the country and target population groups when transferring health innovations from one to another country, or when translating research into policy action. Furthermore, the resources available for reproductive health care should encompass all services across the continuum of care and not only facility-based childbirth. The focus on subsidizing or eliminating user fees at health facilities could have a downside of limiting resources available at health facilities because of a 'crowding out' effect, especially for emergency obstetric care services (Gilson \& McIntyre, 2005). Health care providers could in turn inadvertently limit performance for services that are not prioritized due to the lack of funds (Ridde \& Diarra, 2009). This could make reproductive health care less effective for women. To improve reproductive health care services in SubSaharan Africa, health workers need regular training on essential interventions for safe motherhood with adequate equity-related content.

\section{Statement 2: The wealth-gap in the use of reproductive health care services by women follows a comparable pattern in Sub-Saharan African countries.}

Based on country representative data from Burkina Faso, Ghana, Niger, Nigeria, and Senegal, this dissertation shows that wealth-based differentials in the use of reproductive health care services exist in all countries mentioned (Chapter 5). Countries with similar demographic and policy environments have analogous wealth-gaps in access to family planning information and modern contraception use. Countries with more identical services included in the fee waiver policies have comparable inequality gaps. We also observe a significant wealth-based difference between the groups of women for exposure to family planning information via media and the use of modern 
contraceptives. The countries with a user-fee waiver policy also have a substantial magnitude of inequality gap between the poorest and richer wealth groups. Across countries, the gap in maternal care use becomes less with the comprehensiveness of the user-fee policy in place, i.e., the scope and content of the benefits package covered. A previous study on the coverage of health care services for pregnant women and children across Sub-Saharan African countries, suggests that wealth-related inequality varies across countries and types of health care services (Hosseinpoor et al., 2011). This supports what we observe in Chapter 2.

The wealth-gap implies that disadvantaged women in poor households do not benefit from reproductive health care services as much as advantaged women in wealthier households. We report disparities in reproductive health care service use across countries (Chapter 2, 3 and 5). Between Nigeria and Ghana, the inequality and wealthgap in the use of reproductive health care services are remarkably different, much wider in Nigeria than in Ghana. Relatively, Burkina Faso and Ghana have similar inequality and wealth-gap in service use regarding antenatal visits and facility-based childbirth. In countries with fee waiver policies, the disparity between the user groups is wider for facility-based childbirth than ANC visits. Among other reasons, ANC is more readily available than professional childbirth providers (Galadanci et al., 2010). Nigeria does not have a national policy that exempts women from obstetric care costs (Abimbola et al., 2012; Okpani \& Abimbola, 2015). Burkina Faso's user fee for maternal care policy exempts the indigents from payments and subsidizes service costs (except for ANC and postnatal family planning), and Ghana's policy exempts women from costs at the point of use (Richard et al., 2013; Ridde et al., 2015). In terms of reducing inequalities across socioeconomic groups, the policies, however, appear relatively ineffective to address the wealth disparity in access (Johnson et al., 2016; Obiyan \& Kumar, 2015).

While subsidies or removal of user fees for service may increase utilization among pregnant women, the effect on equity is marginal and not consistent across SubSaharan African countries (Chapter 2 and 4). The effect of pro-poor programs that we find, is not necessarily beneficial for the disadvantaged groups or households, which is in line with previous findings from Sub-Sahara African countries. In particular, countries with pro-poor programs, like user fee waivers, still have persistent inequalities in service use (Chapter 2). For example, in Ethiopia, reproductive health care is categorized as a high priority and there is a free maternity services package, a fee-waiver system for the poor and strong family planning programs (Pearson, Gandhi, Admasu, \& Keyes, 2011; Ross, 2015). Differences in wealth quintiles contribute to the wealth-related inequalities in reproductive health by $59 \%$ for family planning, by $48 \%$ for ANC, and by $32 \%$ for skilled birth attendance from previous use (Onarheim et al., 2015). In 
addition, a study on free facility-based childbirth in Ghana, Senegal, and Sierra Leone reported an increase in facility deliveries across all categories of household wealth but insignificant equity effects across wealth groups (McKinnon et al., 2015). Evidence from a study in Mali also shows that the free $\mathrm{C}$-sections initiative benefits women with higher socioeconomic status (El-Khoury et al., 2012). The better-offs benefit more from initiatives to address inequalities in access to health care services, sometimes increasing the inequalities gap (Victora et al., 2018). Furthermore, there is a lack of consensus about the suitability of user fees abolishing policies to reduce inequalities (Hatt et al., 2013; Ridde \& Morestin, 2011). The evidence in support of eliminating/ subsidizing user fees for reproductive services is stronger for surges in the utilization of services rather than moving towards equity.

There is, though, a consensus on the individual factors that determine the use of reproductive health care services (Abekah-Nkrumah, 2018; Ahmed et al., 2010; Creanga et al., 2011; Gillespie, 2007). In this dissertation, we have highlighted that maternal characteristics explain the pattern of wealth-disparity in the use of reproductive health care services (Chapter 5). As expected, maternal characteristics substantially add to the wealth inequality in service use throughout all countries. Individual maternal characteristics foster or hinder getting to the health facility where a user can then take advantage of available exemption policies for reproductive health care (Chapter 4 and 5). We observe strong significance and uniformity in the contribution of maternal characteristics, which explain the disparity in reproductive health care use across all countries. These characteristics include a lack of information on pregnancy complications, living in rural residence, religion, lack of autonomy in health facility seeking decision, need to pay, and distance (Chapter 5). In a study looking at the effect of family planning efforts in relation to fertility decline across the countries in the African region, the socioeconomic advantage is reported to be trivial for fertility decline (Garenne, 2018). However, we find that on top of the contribution of wealth, the contribution of maternal characteristics to the gap in the use of reproductive health care services is non-trivial and comparable.

Exemption policies for user fees at the point of delivery of essential health care services sometimes fall short of their objectives for diverse reasons. In particular, countries that lack political determination or encounter strong tradition and religious resistance would find it hard to assure that such initiatives are effective (Garenne, 2018). Other factors influence the success of reproductive health care initiatives, namely political stability, economic growth, and receptivity of the population, to mention a few examples (Garenne, 2018; Onwujekwe et al., 2016; Ridde \& Diarra, 2009). The user fee 
exemption policies will perform better in a stronger health care system. Specifically, such a health care system allows for the utilization of health care services when needed, and ensures maximum benefits for individuals, with robust policy leadership to identify problems and opportunities and protect individuals. Although Ghana's GDP per capita (US\$1,298.4) is less than Nigeria's (US\$2,292.4) (World Bank, n.d.), the health care system appears to be stronger than that of Nigeria (Asaolu et al., 2019; Odeyemi \& Nixon, 2013). Geographical size could also play a role. However, governance and political factors would matter more (Garenne, 2018). User fee exemptions, if inadequately funded, may result in a reduction in resources available for health care providers and may produce unmet needs as a result of increased utilization of care services (Gilson \& Mclntyre, 2005).

In Chapter 2, we show that Cameroon, Ethiopia, Nigeria, Ghana, and Zimbabwe have comparable constructs of inequality in reproductive care service. Specifically, in addition to higher pro-wealthy inequalities (i.e. more frequent service use by wealthier women), these are the only countries with pro-wealthy inequalities for unskilled childbirth. In addition, Malawi, Namibia, Rwanda, and South Africa follow a comparable inequalities pattern. Specifically, relatively lower pro-wealthy inequalities for skilled childbirth services, ANC services, four or more ANC visits, inadequate ANC components, and postnatal care services. Where there is a health care system with low public health care expenditure and high out-of-pocket payment, waivers/removal of user fees for reproductive health care services would not matter much, especially for poor people. This drives women to use familiar services usually of inadequate quality (Chapter 3). By 2005 and 2010, countries like Burkina Faso, Ethiopia, Ghana and Rwanda (2010 only) had between $20-40 \%$ out-of-pocket payment as a share of total health expenditure in the country, while Cameroun, Ivory Coast (Cote d'Ivoire), Niger, Nigeria, and Sierra Leone had more than $40 \%$ out-of-pocket payment as a share of total health expenditure (WHO, 2013b). Out-of-pocket payments in Malawi, Namibia, and South Africa were less than 20\% of total health expenditure between 2001 and 2010 but Malawi moved from this group to $20-40 \%$ by 2010 (WHO, 2013b). When a fee waiver policy is not properly designed and implemented, service providers in SubSaharan African countries are able to request users to pay out of pocket for services, for example was the case in Burkina Faso, Ethiopia, and Senegal (Pearson et al., 2011; Ridde et al., 2015; Witter et al., 2010). When needs for care among a population are unmet, it leads to disparity in health care utilization (Gulliford et al., 2002).

We conclude that addressing the socioeconomic barriers to reproductive health care service use through supply-side policies is not a substitute for supportive policies 
needed to address an increased service utilization as well as the resulting wealth disparities. The inclusion of user fee waiver policies for maternal care services alone does not increase equity in the use of reproductive health care services in Sub-Saharan African countries. This is especially the case since most policies do not (appropriately) distinguish between women previously benefitting from services covered from those who are not benefiting (Pearson et al., 2011). In Chapters 4 and 5 of this dissertation, we argue that there remains a wide wealth gap in reproductive health care services use in countries with policies addressing the cost of services and countries without such policies. This suggests that more wealthy households do not essentially allocate more personal resources for health care but also take advantage of the policy environment that creates equal opportunities for reproductive health care access. Fee waiver policies that do not address other maternal characteristics and inequalities in opportunities for reproductive health care services will continue to maintain the wealth gap in use.

\section{Research and policy implications}

The discussion above shows that inequity in the use of reproductive health care is not limited to socioeconomic or wealth barriers and constraints encountered at the point of service use. Distribution of resources and the implementation of initiatives have equity effects as well. Further research, using observational study data, could provide context-specific evidence on differences in maternal characteristics between advantaged groups (benefitting from a fee waiver policy or able to afford the cost of services) for reproductive health care services and other groups with no policy exposure. Additional mixed methods studies are needed to assess the equity effect on the disadvantaged groups, e.g. What maternal characteristics should be the focus of pre-pregnancy, pregnancy, childbirth, emergency obstetrics, and postpartum services. The design of practical programs and targeted evidence-informed applications of policies would benefit from this type of research.

To narrow the wealth-gaps in the use of reproductive health care services by women, resources should be focused and used to address the most vulnerable population needs. Existing interventions should be assessed and if necessary redesigned to embed an equity focus in countries already at the implementation stage. This can be done by making equity a national and State health priority, and by having a comprehensive approach towards equity in health care service at all stages of policy processes. Thus, initiatives will capture reducing inequalities in health outcomes or access to quality health care between different socioeconomic groups, or maintaining an essential minimum level of health for all. To broaden the scope and coverage of reproductive health care exemption packages, policymakers need to consider other means to 
generate funding for health care, such as cross-subsidization.

\section{Statement 3: Wealth-based inequalities in the use of reproductive health care services by women in Sub-Saharan Africa, sustain over time because poor women in this region are not adequately targeted for additional support in population-wide health programs.}

Policies on reproductive health are needed to reduce negative health outcomes. A number of policies for maternal health care in Sub-Saharan African countries address out-of-pocket payment for care services through total or partial fee waiver at point of use (De Brouwere et al., 2010; Ridde \& Morestin, 2011). Other strategies used, also those that address family planning services, include the provision of human resources, like community health extension workers and midwives in resource-poor settings (Abimbola et al., 2012; Exley et al., 2016; Medhanyie et al., 2012; Nyonator et al., 2005; Olaniran, Madaj, Bar-Zev, \& van den Broek, 2019; Phillips et al., 2005). These population-wide strategies aimed at an entire population group of women for improved reproductive health, can provide an increase in health care utilization and address the immediate need for services. Also, these national approaches are important strategies to provide skilled personnel and services in remote areas and to lift the financial burden for reproductive health care services (El-Khoury et al., 2012; Hatt et al., 2013; Ravit et al., 2018; Ridde et al., 2015; Witter, 2009; Zere et al., 2007).

In Chapter 4, we observe, over years and across socioeconomic groups, that most indicators of reproductive health care services use in Ghana indicate a move towards more equity, albeit slightly. This improvement in use towards more equity was not observed for essential services like assistance by a physician during childbirth, having ANC at government and private facilities. In Nigeria, we note that equity changes in the use of reproductive health care services over the years are unfavorable to women in poor households. Thus, poor women in diverse social groups remain at a disadvantage of adequate reproductive health care service use and wealthier women benefit from these services more. Countries with national maternal health care policies like Burkina Faso, Ethiopia, Ghana, and South Africa have appreciable narrow inequalities and lower wealth-based gaps, unlike Nigeria and Senegal with limited regional population coverage (Chapter 2 and 5). The overall use of reproductive health care increases in countries where all geographical areas are covered but are skewed in favor of wealthier households. The conventional hypothesis is that new interventions will eventually diffuse to disadvantaged groups after the wealthier population has almost total coverage (Victora et al., 2000). However, our study of the trend in inequality in Ghana and Nigeria (Chapter 4) shows that reproductive health care interventions do 
not progress to the point they eventually benefit the poorer populations absolutely. Rather they stagnate after a certain period of time after the implementation.

Our study on trends in Ghana and Nigeria (Chapter 4) also suggests that lengthier reproductive health interventions are able to influence service use but the variation among social groups continues. Thus, narrower and persistent inequalities exist in countries with a fee waiver. For example, Ghana has applied a fee exemption policy for antenatal services since 1963 (Koduah et al., 2015) and South Africa has provided free health care for pregnant women in 1994 (Schneider \& Gilson, 2006). Population-wide health interventions provide a boost in reproductive health care use and address needs at the point in time, but they tend to widen and maintain inequalities (Johnson et al., 2009; Mezmur et al., 2017; Schneider \& Gilson, 2006).

Across social groups, reproductive health care services use remains unfavorably distributed with minimal changes over the years (Chapter 4). Across social groups of women with equal health care needs, women of similar age, marital status, occupation, area of residence (rural or urban), and region of residence, the unequal use of quality reproductive health care services has continued (Chapter 4 and 5). Other barriers to equitable care that contribute to socioeconomic inequality and sustain unequal treatment of women with similar needs include the lack of information on pregnancy complications, religion, need to pay, distance, and a lack of autonomy to seek health care at a facility (Chapter 4 and 5). Where people cannot (are unable to or not allowed to) make use of health services, such policies only benefit advantaged groups, who can get to the point of service delivery. Government-sponsored initiatives for reproductive health, have not been beneficial to poorer women because they are too general. A general policy does not specifically aim to reduce or eliminate health differences which result from factors considered to be avoidable and unfair (Whitehead, 1992). Since reproductive health care initiatives are sporadically funded and lack a long-term strategy, shifting periodically, (Koduah et al., 2016), they are seldom appropriately monitored and evaluated to efficiently provide direction for future intervention activities (Richard, Witter, \& de Brouwere, 2010).

In addition, the choice of a policy adopted by policymakers is influenced by different policy motivations and visions on how to implement them. The nature of agendasetting and funding patterns for reproductive health care is also a reason for the continued inequality pattern we observe. This, for example, resulted in the removal of family planning from the maternal health policy and program in Ghana despite the consciousness of its benefits for maternal health - funds for contraceptives were allocated later in the same year 2003 (Koduah et al., 2016). This temporarily 
paused the implementation of Ghana's maternal user fee exemption policy between 2005 and 2006 (Koduah et al., 2016). In resource-constrained environments, it is understood that when policymakers do not have all the information to choose the most effective intervention, one is chosen until the budget is exhausted (Baltussen \& Niessen, 2006). As such, initiatives are implemented with hopes of securing funds which results in intervention stagnation (Abimbola et al., 2012; Nyonator et al., 2005; Ogbuabor, Onwujekwe, \& Ezumah, 2019; Reichenbach, 2002). This method of policy process perpetuates inequality in the use of reproductive health care services among disadvantaged groups. Fee waiver policies need to "[allow] for the sustained mobilization of resources to achieve human rights to health, along with health equity goals" (Gilson \& Mclntyre, 2005, p. 764).

Poor women are not only limited by socioeconomic status but also by the social groups they belong to or identify with. These limitations refer to age, marital status, occupation, place of residence (rural or urban), and region of residence. The wealthbased gap is unequal across sociodemographic groups. Our results also contribute to the understanding of how poor women can be identified for additional support in countries since health care system characteristics varies across Sub-Saharan African countries (Chapter 4 and 5). As shown in this dissertation, sociodemographic factors explain at least $40 \%$ of the inequality between the wealth groups in exposure to mass media family planning information, $30 \%$ in modern contraceptive use, $24 \%$ of adequate ANC visits, $47 \%$ of the difference in facility-based childbirths, and $62 \%$ in $\mathrm{C}$-section (Chapter 5). Hence, there are some variations in the magnitude of the different factors across countries. Although social determinants of inequality health disparities are noted in previous literature (Braveman, 2003; O'Neill et al., 2014), policymakers are fixated on population-wide interventions for reproductive health care that address the shortcoming in service use through fee waivers (Hatt et al., 2013). This attentiveness is seldom effective in social groups with different social advantages and diverse needs. For instance, the implementation of the NHIS in Nigeria did not benefit women in the informal sector occupation who have higher needs (Obiyan \& Kumar, 2015). Implemented community-based programs have not proven to be useful for equitable delivery of quality services or for a response to social determinants of health (McCollum, Gomez, Theobald, \& Taegtmeyer, 2016).

Programs for reproductive health care in resource-poor settings in most SubSaharan African countries, have short life spans and are inconsistently executed. However, targeting disadvantaged groups will be a strategy to reduce inequalities in reproductive health care services use in the short term but not to close the disparity 
in use. The heavier burden of negative reproductive health falls on the disadvantaged groups, which the national health policies do not cater to, as seen in their inability to substantially improve the use of services. These population-wide policies for women are broadly worded with implementation gaps (Galadanci et al., 2010; Witter et al., 2007). This results in the denial of services to the poor since informal fees are being charged despite free health care for pregnant women policy (Schneider \& Gilson, 2006). Country-level initiatives that have deployed resources nation-wide or to poor communities, leave out groups with lower levels of social advantage through the inability to provide services due to individual factors preventing access in such a community. Religion, for example, may prohibit the use of family planning services (Gyimah, Takyi, \& Addai, 2006).

In conclusion, current reproductive care policies do not provide equal opportunities for health services use in Sub-Saharan African countries among women in different socioeconomic status and with individual characteristics. Reproductive health care programs being implemented in most Sub-Saharan African countries have short life spans and are inconsistently implemented. This suggests that country-wide interventions sustain inequalities since the characteristics of poor women are not addressed initially in reproductive health care packages and cease before they achieve coverage. These health interventions are able to provide temporal improvements in reproductive health and address the point in time need for services. Time-limited initiatives do not have in-built sustainability or opportunities to scale up to meet the health care needs of disadvantaged groups (Koblinsky et al., 2006). This erodes the gains made over time before the disadvantaged benefit. Many barriers to equitable use of reproductive health care services persist. Removing user fees is a first step that many Sub-Saharan African countries, such as Ghana, have sustained for decades. The social determinants of inequality and those characteristics that ensure continued inequality among women with equal needs are critical alongside sustainable fee waiver policies (Chapter 4 and 5). Evidence suggests that population-wide and top-down public policies without financial sustainability strategy do not benefit the poor (Richard et al., 2010).

\section{Research and policy implications}

To sustainably reduce inequalities in the use of reproductive health care services across the wealth groups in Sub-Saharan African countries, there is a need to identify and target characteristics of disadvantaged groups of women in population-wide interventions. Some clusters of women in their reproductive ages are unable to benefit from available health care services, despite national policies that create such opportunities. Although fee waiver policies are useful for service utilization, especially 
when services are available, more evidence is needed on the contextual barriers to the implementation of demand-side programs. Future research can provide further details on the sociodemographic characteristics of women, stratified by economic status, regarding reproductive health care services in their locality. This will identify attitudes, barriers, motivations, and communication between clients and providers, and the organization of the geographical locality. This will provide real-world evidence for policymakers and health service planners to make context-appropriate interventions that effectively target disadvantaged groups within their health care environment.

Based on the results discussed, there is evidence of sustained inequalities as a result of health care systems and policies in Sub-Saharan African countries. Inequalities in adequate reproductive health care services persist over the years among women in disadvantaged social groups. Nevertheless, the reduced inequality in countries with national fee waiver policies for reproductive health care use, like Ghana, should be recognized when comparing with a country with sporadic geographically-limited user-fee exemption like Nigeria (Chapter 2 and 4). User fee waivers or exemption are important to increase utilization. They can, however, have negative effects on increasing the wealth-based differences in the service use as well as disparity across groups. Regarding the significance for countries planning to implement a policy waiver for reproductive health care, the sources of disparity in the use of reproductive health care services should be captured in pilot stages. All countries with starting or with on-going exemptions should implement interventions bottom-up. Intervention targeting the most disadvantaged would address multifaceted barriers to access reproductive health care services rather than the single barrier of user-fee for service in Sub-Saharan Africa.

\section{Statement 4: The reproductive health care interventions in Sub-Saharan African countries need to be structured, evidence-driven and adequately monitored to be able to narrow the inequality gap in use of services and sustain the gains made.}

The trend analysis based on data for Ghana and Nigeria in Chapter 4 indicates a continuous pattern of inequality with no real gains in narrowing the gaps between the social groups over the years. Moreover, we find in Chapter 5 that maternal characteristics contribute to the wealth-based disparity in the use of reproductive health care services in other countries as well, despite the fee waiver policies. Women encounter various barriers of access to reproductive health care services, out-of-pocket payment at a service point is one of them. We show that pro-poor reproductive health care policies, usually in the form of fee-waivers for service users do not sufficiently address contextual factors, which are significant barriers to access. Also, adequate reproductive health care services are not equally available across social 
groups (Chapter 3). We observe a high level of poor access to maternal health care in Central, Northern, and Upper West regions, three of the four poorest regions of Ghana where user fee exemption for maternal care was piloted before the scale-up (Chapter 3). Living in some regions, such as the Northern region in Ghana, contributes to the wealth-based gaps in reproductive health care services like facility-based childbirth services and $\mathrm{C}$-section (Chapter 5). As already discussed above, policies addressing reproductive health care do not necessarily provide disadvantaged women with needed services or leverage to access services, if these policies are not evidencedriven and adequately monitored. This, however, is not always the case in Sub-Saharan Africa. The process of priority setting and resource allocation for maternal health in Sub-Saharan African countries has been referred to as an "arbitrary basis for selecting high-priority services for exemption" (Witter, 2009, p. 284).

The narrowing of inequalities in the use of essential reproductive health care services across groups dissipates over time (Chapter 4), although progress in the use of such services has been noted in some Sub-Saharan African countries. The lack of structured reproductive health interventions results is an implementation deficit where organization and delivery of care services are dependent on the will of stakeholders to make changes (Agyepong \& Adjei, 2008; Ogbuabor et al., 2019). In Sub-Saharan Africa, policymakers often represent their own interests in a policy agenda and therefore, improvements in equity and quality of reproductive health care services can be said to be path-dependent (Koduah, Agyepong, \& van Dijk, 2018; Ogbuabor et al., 2019). This means that policies are a result of nonreversible processes that have similar starting points or same guiding rules but have different, nonlinear outcomes dependent on the initial conditions and different choices made along the way (Bennett $\&$ Elman, 2006; Paina \& Peters, 2012). An example is Ghana's policy of free care services for maternal care, which has been in place since 1963, as well as the safe motherhood initiative implemented in 1995, and free childbirth care policy implemented in 2003 and scaled up in 2005, which resulted in free maternal care policy in 2009. Although these policies are considered to be formed based on evidence, stakeholders interpreted the evidence using their discretion, position, legal and structural authority, control and access to resources, among others, to negotiate support for policy agenda items (Koduah et al., 2015). Similarly, there was the free maternal and child health policy in Nigeria between 2008 and 2015, but it was only implemented in selected states of Nigeria (Onwujekwe et al., 2016). In Nigeria, states like Cross-River, Enugu, Kano, at different points implemented a user fee exemption for maternity care to improve the use and eliminate catastrophic out of pocket payments (Edu et al., 2017; Okafor, Obi, \& Ugwu, 2010). The free service path has not transformed the use of care even though the 
contextual factors and stakeholders have changed. Stakeholders in the health sector need to embrace uncertainty, nonlinear processes, peculiar local context, emergent characteristics, and intentional adaptation for such fee waiver policies so that such policies become effective (Ogbuabor et al., 2019).

Decision-makers have access to, and sometimes use, evidence to promote their ideas on reproductive health care policy (Nyonator et al., 2005; Phillips et al., 2005). However, this evidence is often not adapted to a specific context, missing the opportunity to genuinely address inequalities and social group disparities. Transfer of techniques from one society to another requires the inclusion of the context factors and an understanding of the capacity needed to adapt to serve a particular setting. Often, items on the reproductive health agenda are a direct duplicate of previous intervention, or of interventions from another context, such as the community-based health insurance schemes, fee waiver policies, or community health workers program. Overall, reproductive health care interventions have been designed and implemented because it is deemed politically appropriate, and to fulfill political promises (Ogbuabor et al., 2019; Rudan et al., 2010). There is little doubt that these policies were not born out of scientific and economic evidence since there are rarely feasibility studies conducted or baseline data to guide intervention design or implementation (Ganle, Parker, Fitzpatrick, \& Otupiri, 2014; Kendall \& Langer, 2015; Onwujekwe et al., 2016; Rudan et al., 2010). An example is Ghana's national health insurance scheme implemented after the incumbent won the 2004 election (Koduah et al., 2016).

There are also conscious decisions by policymakers not to proceed with a line of action for which the decisions are not based on evidence. An example is Ghana's removal of a proposed needs assessment baseline for basic as well as comprehensive emergency obstetric and newborn care, and the national reproductive health strategic plan (Koduah et al., 2016). Also, in Nigeria, the safe motherhood initiative became relevant among policymakers in 1999 due to increasing attention to maternal mortality and the accumulation of evidence on national-level maternal deaths (Shiffman \& Okonofua, 2007). This relevance, however, rarely translated to practical actions, especially at the State level. The lack of reliable evidence on maternal health makes it possible for subnational officials to deny that there is a problem. Moreover, government officials out of political self-interest also do not find it necessary to make safe motherhood a political priority (Onwujekwe et al., 2016; Shiffman \& Okonofua, 2007).

Health care initiatives that seek to address the wealth-related inequity in access and utilization of reproductive health care services - usually, maternal mortality - have 
been insufficient and ineffective. Most of the countries in Sub-Saharan Africa rely on these estimates from the DHS to inform on sociodemographic and socioeconomic differences in access patterns. Evidence from indices provided from such surveys, like the number of family planning service delivery points, contraceptive use by types, number of ANC visits, and facility-based childbirths in the population, have been used to decide priorities (Koduah et al., 2016; Wallace \& Kapiriri, 2019). Policy decisions on such crude numbers do not capture the social effect at the individual level or best strategies to reduce health inequalities between population subgroups. The use of priority-setting tools in resource-poor settings is imperative to improve decisionmaking for health care including reproductive health policies (Rudan et al., 2010; Wallace \& Kapiriri, 2019). Policies have been decided on the basis of need or political reasons, rather than on the greatest capacity to benefit disadvantaged social groups. Moreover, the monitoring of the health care system is important to understand changes in disparities between social groups and changes over time (Braveman, 2003). This will provide information about the significance of important sociodemographic factors that determine reproductive health care services utilization. Initiatives for reproductive health are usually not well-documented and assessed to show evidence of substantial programmatic success/impact for replication or further studies. Though the proposal to create a baseline needs assessment was made in the maternal health policy and program by policymakers in 2010 in Ghana, it was left out (Koduah et al., 2016). A needs assessment baseline would have facilitated a context-appropriate initiative to determine high-priority services and for those in need, as well as to make improvements and allocate reproductive health care resources more efficiently. A structured and consistent approach, as well as monitoring of equity in health, is important to understand the impacts of policy on the less-advantaged groups (Braveman, 2003). This will improve information about peculiar local contexts, emergent characteristics, for intentional adaptation of alternatives being selected not necessarily due to constrains by policymakers to keep to the particular path.

Since there are rarely economic evaluations to inform on the feasibility, efficiency, and effectiveness of reproductive health care strategies in Sub-Saharan African countries, reproductive health care policies make use of crude evidence of numbers. This policymaking strategy does not ensure that scarce resources are targeted to where they have the greatest effect, that is, the most disadvantaged. There is a need for decision-makers to move to economic evaluations for priority setting and resource allocation for reproductive health care services in Sub-Saharan African countries. In conclusion, the current reproductive health care policies, either the fee waiver for specific or all maternal health services or the community-based services, neglect 
peculiar local contexts in their designs (Koduah et al., 2015; Ogbuabor et al., 2019; Pearson et al., 2011). The interactions of actors in a policy process outcomes such as health inequities, which result from, and might persist over time due to, "nonlinearity of processes and institutional changes during policy implementation" (Ogbuabor et al., 2019, p. 683). Stakeholders in health should move beyond linear thinking and explore a wider range of possible outcomes. In this dissertation, we advocate that to bridge the inequality health gap between social groups, actors in health care need to embrace the uncertainty and unpredictability of outcomes of policies in policy decisions. There are indications that decision-makers in Sub-Saharan African countries do not usually adopt useful and relevant information from research, thereby missing opportunities for adaptation.

\section{Research and policy implications}

As indicated by the results of the quantitative analysis of our case study countries, Ghana and Nigeria, and other Sub-Saharan African countries, inequities in the use of reproductive care exist and persist over time. However, countries with longer ongoing fee exemption policies have comparable inequality gaps that appear to stall. There is a need for current health care reforms to treat the health care delivery system as a complex adaptive system. Future studies may examine how insights from complex adaptive systems could support reproductive health care decision-making and allows stakeholders to factor in the key underlying causes and relations. Furthermore, reproductive health care evidence needs to move beyond the numbers towards values. Policies will benefit from insights provided from economic evaluations related to the quality of life, which can help to improve equity.

For example, social welfare functions have a lot of benefits to address variations in health care spending and overcome unfair variations in access taking into account resource constraints.

If health inequalities are reduced, stakeholders in health care need to move from the single path-dependent fee waivers for maternal health and acknowledge uncertainties. Placing an issue on the policy agenda, formulating policies and instruments for appropriate action, and then implementing action effectively to improve health status should be evidence-based. Particularly in the Sub-Saharan African region, policies do not usually reflect realities. Across Sub-Saharan countries, there is a strong need for evidence to be generated and incorporated to inform on organizational and financial policies, resource allocation and patterns of services, and the use of resources by practitioners in delivering patient care. Decision-makers in this region need to move 
from traditional decision-making approaches in health care to applying complex adaptive systems principles.

Statement 5: To be effective, targeted fee exemptions policies for poor women need to address both coverage gaps and equity gaps.

This dissertation examined inequity in reproductive health care services use in SubSaharan Africa, taking Ghana and Nigeria as case studies, and explored the coverage gaps in reproductive health care services. Policies for reproductive health care services have not addressed coverage gaps and equity issues from pre-pregnancy through postpartum care. As pointed out in Chapter 2 of this dissertation, childbirth services have larger wealth-based inequalities in use than other reproductive health care services. We also show that the fee waiver policies for maternal health care in Sub-Saharan African countries do not adequately address variations in health services need and use among population subgroups (Chapters 4 and 5). This results in unfair differences in the pattern of reproductive health care services use among equals with equal needs. Trade-offs in making exemption policies have not been evidence-based. Other studies analyzing the benefits of user fee removal for selected reproductive health care services on inequalities, also observed similar disparities in availability and use across Sub-Saharan African countries (Do et al., 2015; Johnson et al., 2016; McKinnon et al., 2015). The language of the user fee waiver policies typically mentions exemption for all pregnant women (and sometimes children under five), it does not distinguish differences in need and opportunity for access among potential users. The policy content is customarily developed out of the understanding based on needs defined by the burden of maternal mortality (Koduah et al., 2016; Okonofua, Lambo, Okeibunor, \& Agholor, 2011; Rosenfield \& Maine, 1985; WHO, 2015b). Reproductive health care priorities and policy content are then decided based on data of negative reproductive health outcomes, mainly maternal mortality, and difficulties associated with targeting services (Witter, 2009). The priorities set do not reflect the unequally spread burden of reproductive ill-health across socioeconomic and sociodemographic groups neither is it a true reflection of needs. For instance, initiatives for safe motherhood have been prioritized top-down by political leaders in the absence of key stakeholders including researchers and advocacy groups (Shiffman \& Okonofua, 2007). In this way, health policies are unable to deliver evidence-based and context-appropriate reproductive health care services to address the causes of maternal deaths at scale and sustain coverage and quality over time.

There are user fee subsidies or exemptions for pregnant women based on occupation, such as in Nigeria through the national health insurance scheme (Odeyemi \& Nixon, 
2013), based on geographical location (Galadanci et al., 2010; Ogbuabor et al., 2019; Okafor et al., 2010). Other similar exemptions could be nation-wide such as the subsidy policy for maternal care in Burkina Faso (Ridde et al., 2012), in Niger (Ridde \& Diarra, 2009) and Ghana (Witter et al., 2007). The policies, however, do not adequately address the gaps in coverage of reproductive health care services. There is no consideration for subgroups of the population who for example have a different reproductive health care need, who do not have easy access to health services, be it as a result of culture or quality of care. For example, a woman with an unintended pregnancy who does not plan to carry the pregnancy to term could have cultural barriers and is not covered in the exemption policy. Unsafe abortion results in hemorrhage, sepsis, peritonitis, and other reproductive tract infections (Ezeh et al., 2016). A previous study in Benin and Mali did not detect progress in urban-rural inequalities, or socioeconomic inequalities in access to $\mathrm{C}$-section and facility-based childbirth after the introduction of the free C-section policy (Ravit et al., 2018). The coverage of quality health care services for reproductive health care is stunted in areas where the disadvantaged reside. In essence, the policymakers decide that women of reproductive ages, only when pregnant, need reproductive health care services and therefore provide selected services for a fee exemption for maternal health care services. The policy also suggests that pregnant women can use these free/subsidized services wherever they are available since the main barrier, out-of-pocket payments at the point of service use, has been eliminated. Essentially by limiting who and what is covered, as well as the possibility of using other reproductive health care, needs are being met. The present fee exemptions policies are not only narrow but are limited to where services can be used not necessarily where services are needed. There is a need to address the gaps in coverage of services in terms of what is available, and where it is available.

In Chapter 5, we identified the attributes that, in addition to wealth differences, create equity gaps in the use of reproductive health care services, like modern contraceptives and adequate ANC services between groups. The results show gaps in services use between women in similar situations with equal needs. This inequalities across population subgroups are avoidable as they are influenced not just by the socioeconomic differences, but also by social stratifiers, which results in socially unfair differences in the use of reproductive health care services across population groups. As described in Chapter 5, the ANC services and facility-based childbirth are free of charge in Ghana, there is a free childbirth care and C-section policy in Senegal, as well as subsidized maternal health care in Burkina Faso. Still, there are relevant sociodemographic characteristics that create a disparity in the ability to access these free/subsidized reproductive health care services suggesting a high coverage gap 
among women, particularly among disadvantaged households. Policies that are propoor in design do not go further to address the differences in health care services coverage. Under-provision of reproductive health care services is extensive among disadvantaged populations (Zere et al., 2012).

Fee exemption for maternal health care services being implemented in Sub-Saharan African countries like Ghana, and intermittently in some parts of Nigeria, may not guarantee access to quality reproductive health care services if resource allocation does not incorporate equity considerations. We show that socioeconomically disadvantaged women with specific sociodemographic characteristics do not benefit from these fee waiver policies for maternal health care (Chapter 4). Moreover, most policies on reproductive health care in Sub-Saharan African countries have exemptions based on being pregnant or recent childbirth, up to a few weeks. Targeting women who live in disadvantaged areas, with particular cultural or religious affiliation, who lack information on pregnancy issues and have reduced health facility seeking autonomy, can be seen as a source of disparities in the use of reproductive health care services among women of different socioeconomic status (Chapter 5). These individual characteristics exert more significance among poorer women than betteroffs due to their added contribution on top of wealth disparity. The coverage gap also further enshrines this unfairness in use due to health care system characteristics, like the unavailability of quality reproductive health care services irrespective of the social group. We see in Chapter 5 that parity reduces the wealth gap in the use of modern contraceptives but increases the wealth gap in C-sections in countries like Nigeria, Ghana, and Senegal. This reflects that the pro-poor fee exemption policy such as that in Senegal does not effectively address the poor. Other studies have shown that the number of children a woman has contributes to the disparity in facility-based childbirth among women in Ghana and Rwanda (Do et al., 2015). Another study has shown that women with higher parity tend to live in remote areas and use facility care less often (Ayanore et al., 2016a).

Such pro-poor policies also need to address systemic health and social inequities in the attempt to overcome barriers across population groups. These differences prevent women from benefitting from pro-poor reproductive health care interventions and create disparities in access to reproductive health care services across population groups (CSDH, 2008). However, we explained that a stronger health care system has improved available quality reproductive health care services (Chapter 3) and cushioned some of the effects of financial barriers at the point of service use (Chapter 4). Therefore, the coverage of services is more improved in a health care system like 
Burkina Faso, Ethiopia, and Ghana with fee exemption for more reproductive health care services when compared with Niger, Nigeria, and Sierra Leone. The coverage gap, however, exists in all these countries, more in the former than the latter, since total coverage for reproductive health care services is not achieved.

In conclusion, countries with or without full fees for service waiver policies, do not implement strategies that specifically target peculiar characteristics of individuals/ groups with equal health care needs but unequal access to health care (El-Khoury et al., 2012; Witter, 2009). If these social determinants are addressed, the use of essential reproductive health care services will be on the same level as the advantaged counterparts - usually wealthier women. At the same time, the overall service coverage should be addressed as well. Such a combination of policies will essentially improve the coverage and equity gaps simultaneously.

\section{Research and policy implications}

Fee exemption policies in Sub-Saharan Africa are not universal and difficult to sustain in the long term since resources are constrained by funding (Witter, 2009). The effect on the coverage gap indicates that if equity issues are not addressed, the potential to improve service coverage may not be realized. The implication on equity is also limiting due to the inverse care law, where the most disadvantaged with greater needs have the least access to care. Pro-poor policies for women in their reproductive years should not be arbitrary but should be informed based on the best available evidence on their effectiveness. Access to reproductive health care services is not just limited by point of service use but also by social determinants of health.

Future research should set out to provide evidence that would identify need-based coverage (what should be provided by health services, and for whom). Future research should also set out to provide evidence on how reproductive health care services can be equity-oriented and provided to meet the needs of disadvantaged populations. Especially, this can be achieved by recognizing key dimensions of reproductive health care services for disadvantaged populations and developing reproductive health care equity indicators that can account for the quality, process, and outcomes of care when marginalized populations are explicitly targeted (Browne et al., 2012). Through this, the social determinants of reproductive health care service use inequality can be included as the main priority in service provision. This will facilitate achieving patient-centered care and targets the health determinants of the disadvantaged populations in local contexts (World Bank, 2016). 
The gap in the disparity in the use of available services is as important as the availability of needed health care services. For policies to be effective and leave no one who needs reproductive health care services behind, it is necessary to address both gaps simultaneously. There is a need to measure both coverage gaps and equity gaps to obtain insights about what reproductive health care program is most equitable and effective, and therefore contribute to effective initiatives.

\subsection{FINAL WORDS}

This dissertation has presented evidence that the distribution of reproductive health care services in Sub-Saharan African countries results in use disparities between different socio-economic groups of women. But still, strategies and policies to address the issue, have been ineffective. In reality, in building strong health systems, governments have to start from somewhere, which may not be all-inclusive at the beginning. Thus, some policies increase the inequalities for targeted reproductive health care services between social groups instead of narrowing them. Especially when the utilization of such services increases. In this dissertation, we have shown that individual health determinants enshrine inequalities in the use of reproductive health care services, on top of the effect of socio-economic situations. Ignoring these individual health determinants sustains disparities in the long run. Governments need to be forward-thinking with progress projected into longer terms. Narrowing inequalities will require overarching policies by governments in Sub-Saharan Africa intended for all services in the continuum of reproductive health care services. To ensure effectiveness, reproductive health care initiatives in Sub-Saharan African countries have to be structured, evidence-driven and adequately monitored. Designed in ways that will target characteristics of the population at risk for additional support and address both coverage gaps and equity gaps. Though policy implementation cannot be seamless, the improvements should be evidence-based. 

REFERENCES 


\section{REFERENCES}

Abekah-Nkrumah, G. (2018). Spatial variation in the use of reproductive health services over time: a decomposition analysis. BMC Pregnancy Childbirth, 18(1), 63. doi:10.1186/s12884-018-1695-3

Abimbola, S., Okoli, U., Olubajo, O., Abdullahi, M. J., \& Pate, M. A. (2012). The midwives service scheme in Nigeria. PLoS Med, 9(5), e1001211. doi:10.1371/journal.pmed.1001211

AbouZahr, C., \& Vaughan, J. P. (2000). Assessing the burden of sexual and reproductive ill-health: questions regarding the use of disability-adjusted life years. Bulletin of the World Health Organization, 78(5), 655666

Achana, F. S., Bawah, A. A., Jackson, E. F., Welaga, P., Awine, T., Asuo-Mante, E., . . Phillips, J. F. (2015). Spatial and socio-demographic determinants of contraceptive use in the Upper East region of Ghana. Reprod Health, 12(1), 29. doi:10.1186/s12978-015-0017-8

Adamu, Y. M., \& Salihu, H. M. (2002). Barriers to the use of antenatal and obstetric care services in rural Kano, Nigeria. J Obstet Gynaecol, 22(6), 600-603. doi:10.1080/0144361021000020349

Aday, L. A. (1975). Economic and noneconomic barriers to the use of needed medical services. Med Care, 13(6), 447-456. doi:10.1097/00005650-197506000-00001

Aday, L. A., \& Andersen, R. (1974). A framework for the study of access to medical care. Health Serv Res, 9(3), 208-220.

Addai, I. (2000). Determinants of use of maternal-child health services in rural Ghana. Journal of Biosocial Science, 32(1), 1-15.

Adeyanju, O., Tubeuf, S., \& Ensor, T. (2017). Socio-economic inequalities in access to maternal and child healthcare in Nigeria: changes over time and decomposition analysis. Health Policy Plan, 32(8), 11111118. doi:10.1093/heapol/czx049

African Union. (2018). Maputo plan of action 2016-2030 for the operationalisation of the continental policy framework for sexual and reproductive health and rights. Addis Ababa: African Union, 2015. In.

Agence Nationale de la Statistique et de la Démographie - ANSD/Sénégal, \& ICF. (2017). Senegal Enquête Démographique et de Santé Continue (EDS-Continue) 2016. Retrieved from Dakar, Sénégal: http:// dhsprogram.com/pubs/pdf/FR331/FR331.pdf

Agyepong, I. A., \& Adjei, S. (2008). Public social policy development and implementation: a case study of the Ghana National Health Insurance scheme. Health Policy Plan, 23(2), 150-160. doi:10.1093/heapol/czn002

Ahmed, S., Creanga, A. A., Gillespie, D. G., \& Tsui, A. O. (2010). Economic status, education and empowerment: implications for maternal health service utilization in developing countries. PLoS One, 5(6), e11190. doi:10.1371/journal.pone.0011190

Ahonsi, B. (2015). Commentary: Accelerating the quest for integrated and comprehensive sexual and reproductive health services in Nigeria. Glob Public Health, 10(2), 174-176. doi:10.1080/17441692.2014 .986162

Aikins, M., Aryeetey, R., Dako-Gyeke, P., Adongo, P. B., \& McGough, L. (2015). Socio-economic differences in cost of pregnancy-related health services in the peri-urban Accra, Ghana. J Public Health (Oxf), 37(3), 
540-546. doi:10.1093/pubmed/fdu072

Ajaero, C. K., Odimegwu, C., Ajaero, I. D., \& Nwachukwu, C. A. (2016). Access to mass media messages, and use of family planning in Nigeria: a spatio-demographic analysis from the 2013 DHS. BMC Public Health, 16, 427. doi:10.1186/s12889-016-2979-z

Alam, N., Hajizadeh, M., Dumont, A., \& Fournier, P. (2015). Inequalities in maternal health care utilization in sub-Saharan African countries: a multiyear and multi-country analysis. PLoS One, 10(4), e0120922. doi:10.1371/journal.pone.0120922

Alkema, L., Chou, D., Hogan, D., Zhang, S. Q., Moller, A. B., Gemmill, A., . . Say, L. (2016). Global, regional, and national levels and trends in maternal mortality between 1990 and 2015, with scenario-based projections to 2030: a systematic analysis by the UN Maternal Mortality Estimation Inter-Agency Group. Lancet, 387(10017), 462-474. doi:10.1016/S0140-6736(15)00838-7

Allsworth. (2017). OBSOLETE: Populations at Special Health Risk: Women. In Reference Module in Biomedical Sciences.

Amakom, U. (2013). Subsidy Reinvestment and Empowerment Programme (SURE-P) Intervention in Nigeria: An Insight and Analysis.

Amoako Johnson, F., Padmadas, S. S., \& Matthews, Z. (2013). Are women deciding against home births in low and middle income countries? PLOS One, 8(6), e65527. doi:10.1371/journal.pone.0065527

Andersen, R. M. (1995). Revisiting the behavioral model and access to medical care: does it matter? J Health Soc Behav, 36(1), 1-10

Anderson, J. G. (1973). Health services utilization: framework and review. Health Serv Res, 8(3), 184-199.

Anya, S. E., Hydara, A., \& Jaiteh, L. E. (2008). Antenatal care in The Gambia: missed opportunity for information, education and communication. BMC Pregnancy Childbirth, 8, 9. doi:10.1186/1471-2393-8-9

Aremu, O., Lawoko, S., \& Dalal, K. (2011). Neighborhood socioeconomic disadvantage, individual wealth status and patterns of delivery care utilization in Nigeria: a multilevel discrete choice analysis. Int J Womens Health, 3, 167-174. doi:10.2147/IJWH.S21783

Arsenault, C., Fournier, P., Philibert, A., Sissoko, K., Coulibaly, A., Tourigny, C., . . Dumont, A. (2013). Emergency obstetric care in Mali: catastrophic spending and its impoverishing effects on households. Bull World Health Organ, 91(3), 207-216. doi:10.2471/BLT.12.108969

Arthur, E. (2012). Wealth and antenatal care use: implications for maternal health care utilisation in Ghana. Health Econ Rev, 2(1), 14. doi:10.1186/2191-1991-2-14

Asamoah, B. O., Agardh, A., \& Ostergren, P. O. (2013). Inequality in fertility rate and modern contraceptive use among Ghanaian women from 1988-2008. Int J Equity Health, 12(1), 37. doi:10.1186/1475-9276-12-37

Asamoah, B. O., Agardh, A., Pettersson, K. O., \& Ostergren, P. O. (2014). Magnitude and trends of inequalities in antenatal care and delivery under skilled care among different socio-demographic groups in Ghana from 1988 - 2008. BMC Pregnancy Childbirth, 14, 295. doi:10.1186/1471-2393-14-295

Asante, F., Chikwama, C., Daniels, A., \& Armar-Klemesu, M. (2007). Evaluating the economic outcomes of the policy of fee exemption for maternal delivery care in ghana. Ghana Med J, 41(3), 110-117. doi:10.4314/ gmj.v41i3.55277 
Asaolu, I., Nuno, V. L., Ernst, K., Taren, D., \& Ehiri, J. (2019). Healthcare system indicators associated with modern contraceptive use in Ghana, Kenya, and Nigeria: evidence from the Performance Monitoring and Accountability 2020 data. Reprod Health, 16(1), 152. doi:10.1186/s12978-019-0816-4

Ashford, L. (2003). Unmet need for family planning: Recent trends and their implications for programs: Population Reference Bureau.

Awoonor-Williams, J. K., Sory, E. K., Nyonator, F. K., Phillips, J. F., Wang, C., \& Schmitt, M. L. (2013). Lessons learned from scaling up a community-based health program in the Upper East Region of northern Ghana. Glob Health Sci Pract, 1(1), 117-133. doi:10.9745/GHSP-D-12-00012

Ayanore, M. A., Pavlova, M., \& Groot, W. (2016a). Focused maternity care in Ghana: results of a cluster analysis. BMC Health Serv Res, 16(1), 395. doi:10.1186/s12913-016-1654-5

Ayanore, M. A., Pavlova, M., \& Groot, W. (2016b). Unmet reproductive health needs among women in some West African countries: a systematic review of outcome measures and determinants. Reprod Health, 13, 5. doi:10.1186/s12978-015-0104-x

Baatiema, L., Sumah, A. M., Tang, P. N., \& Ganle, J. K. (2016). Community health workers in Ghana: the need for greater policy attention. BMJ Glob Health, 1(4), e000141. doi:10.1136/bmjgh-2016-000141

Babalola, S., \& Fatusi, A. (2009). Determinants of use of maternal health services in Nigeria--looking beyond individual and household factors. BMC Pregnancy Childbirth, 9(1), 43. doi:10.1186/1471-2393-9-43

Babalola, S., Folda, L., \& Babayaro, H. (2008). The effects of a communication program on contraceptive ideation and use among young women in northern Nigeria. Stud Fam Plann, 39(3), 211-220. doi:10.1111/ j.1728-4465.2008.168.x

Baltussen, R., \& Niessen, L. (2006). Priority setting of health interventions: the need for multi-criteria decision analysis. Cost effectiveness and resource allocation : C/E, 4, 14. doi:10.1186/1478-7547-4-14

Barros, A. J., Ronsmans, C., Axelson, H., Loaiza, E., Bertoldi, A. D., Franca, G. V., . . Victora, C. G. (2012). Equity in maternal, newborn, and child health interventions in Countdown to 2015: a retrospective review of survey data from 54 countries. Lancet, 379(9822), 1225-1233. doi:10.1016/S0140-6736(12)60113-5

Barros, A. J., \& Victora, C. G. (2013). Measuring coverage in MNCH: determining and interpreting inequalities in coverage of maternal, newborn, and child health interventions. PLoS Med, 10(5), e1001390. doi:10.1371/ journal.pmed.1001390

Bennett, A., \& Elman, C. (2006). Qualitative research: Recent developments in case study methods. Annual Review of Political Science, 9(1), 455-476. doi:10.1146/annurev.polisci.8.082103.104918

Benson, A., Calhoun, L., Corroon, M., Gueye, A., Guilkey, D., Kebede, E., . . Winston, J. (2018). The Senegal urban reproductive health initiative: a longitudinal program impact evaluation. Contraception, 97(5), 439444. doi:10.1016/j.contraception.2018.01.003

Bertakis, K. D., Azari, R., Helms, L. J., Callahan, E. J., \& Robbins, J. A. (2000). Gender differences in the utilization of health care services. J Fam Pract, 49(2), 147-152.

Bhutta, Z. A. (2005). Bridging the equity gap in maternal and child health. BMJ, 331(7517), 585-586. doi:10.1136/ bmj.38603.526644.47

Bhutta, Z. A., Chopra, M., Axelson, H., Berman, P., Boerma, T., Bryce, J., . . Wardlaw, T. (2010). Countdown to 
2015 decade report (2000-10): taking stock of maternal, newborn, and child survival. Lancet, 375(9730), 2032-2044. doi:10.1016/S0140-6736(10)60678-2

Blanchet, N. J., Fink, G., \& Osei-Akoto, I. (2012). The effect of Ghana's National Health Insurance Scheme on health care utilisation. Ghana Med J, 46(2), 76-84.

Bonfrer, I., van de Poel, E., Grimm, M., \& Van Doorslaer, E. (2014). Does the distribution of healthcare utilization match needs in Africa? Health Policy Plan, 29(7), 921-937. doi:10.1093/heapol/czt074

Borghi, J., Hanson, K., Acquah, C. A., Ekanmian, G., Filippi, V., Ronsmans, C., . . Alihonou, E. (2003). Costs of near-miss obstetric complications for women and their families in Benin and Ghana. Health Policy Plan, 18(4), 383-390. doi:10.1093/heapol/czg046

Braveman, P. (2006). Health disparities and health equity: concepts and measurement. Annu Rev Public Health, 27, 167-194. doi:10.1146/annurev.publhealth.27.021405.102103

Braveman, P., \& Gruskin, S. (2003). Defining equity in health. J Epidemiol Community Health, 57(4), 254-258. doi:10.1136/jech.57.4.254

Braveman, P., \& Tarimo, E. (2002). Social inequalities in health within countries: not only an issue for affluent nations. Social science \& medicine, 54(11), 1621-1635.

Braveman, P. A. (2003). Monitoring equity in health and healthcare: a conceptual framework. J Health Popul Nutr, 21(3), 181-192.

Brick, A., Layte, R., Nolan, A., \& Turner, M. J. (2016). Differences in nulliparous caesarean section rates across models of care: a decomposition analysis. BMC Health Serv Res, 16, 239. doi:10.1186/s12913-016-1494-3

Browne, A. J., Varcoe, C. M., Wong, S. T., Smye, V. L., Lavoie, J., Littlejohn, D., . . Lennox, S. (2012). Closing the health equity gap: evidence-based strategies for primary health care organizations. Int J Equity Health, 11(1), 59. doi:10.1186/1475-9276-11-59

Campbell, O. M. R., Graham, W. J., \& group., L. M. S. S. S. (2006). Maternal survival 2 - Strategies for reducing maternal mortality: getting on with what works. Lancet, 368(9543), 1284-1299. doi:10.1016/S01406736(06)69381-1

Canning, D., \& Schultz, T. P. (2012). The economic consequences of reproductive health and family planning. Lancet, 380(9837), 165-171. doi:10.1016/S0140-6736(12)60827-7

Castro-Leal, F., Dayton, J., Demery, L., \& Mehra, K. (1999). Public Social Spending in Africa: Do the Poor Benefit? The World Bank Research Observer, 14(1), 49-72. doi:10.1093/wbro/14.1.49

Committee on Economic, Social and Cultural Rights. General Comment No. 22: on the Right to sexual and reproductive health (article 12 of the International Covenant on Economic, Social and Cultural Rights). In: United Nations. Economic Council.

Chiu, T., Fang, D., Chen, J., Wang, Y., \& Jeris, C. (2001). A robust and scalable clustering algorithm for mixed type attributes in large database environment. Paper presented at the Proceedings of the seventh ACM SIGKDD international conference on knowledge discovery and data mining.

Chow, J. (1998). Differentiating urban neighborhoods: A multivariate structural model analysis. Social Work Research, 22(3), 131-142.

Cisse, C. T., Faye, E. O., de Bernis, L., Dujardin, B., \& Diadhiou, F. (1998). [Cesarean sections in Senegal: coverage 
of needs and quality of services]. Sante, 8(5), 369-377.

Clarke, P. M., Gerdtham, U. G., Johannesson, M., Bingefors, K., \& Smith, L. (2002). On the measurement of relative and absolute income-related health inequality. Soc Sci Med, 55(11), 1923-1928. doi:10.1016/ s0277-9536(01)00321-5

Cook, R. J. (1993). International human rights and women's reproductive health. Stud Fam Plann, 24(2), 73-86. doi:10.2307/2939201

Countdown Equity Analysis, G., Boerma, J. T., Bryce, J., Kinfu, Y., Axelson, H., \& Victora, C. G. (2008). Mind the gap: equity and trends in coverage of maternal, newborn, and child health services in 54 Countdown countries. Lancet, 371(9620), 1259-1267. doi:10.1016/S0140-6736(08)60560-7

Couttolenc, B. F. (2012). Decentralization and governance in the Ghana health sector: The World Bank.

Creanga, A. A., Gillespie, D., Karklins, S., \& Tsui, A. O. (2011). Low use of contraception among poor women in Africa: an equity issue. Bull World Health Organ, 89(4), 258-266. doi:10.2471/BLT.10.083329

Crissman, H. P., Adanu, R. M., \& Harlow, S. D. (2012). Women's sexual empowerment and contraceptive use in Ghana. Stud Fam Plann, 43(3), 201-212. doi:10.1111/j.1728-4465.2012.00318.x

Commission on Social Determinants of Health. (2008). Closing the gap in a generation: health equity through action on the social determinants of health: final report of the commission on social determinants of health.

Darroch, J. E., \& Singh, S. (2013). Trends in contraceptive need and use in developing countries in 2003, 2008, and 2012: an analysis of national surveys. Lancet, 381(9879), 1756-1762. doi:Doi 10.1016/S01406736(13)60597-8

De Allegri, M., Ridde, V., Louis, V. R., Sarker, M., Tiendrebeogo, J., Ye, M., . . Jahn, A. (2011). Determinants of utilisation of maternal care services after the reduction of user fees: a case study from rural Burkina Faso. Health Policy, 99(3), 210-218. doi:10.1016/j.healthpol.2010.10.010

De Allegri, M., Ridde, V., Louis, V. R., Sarker, M., Tiendrebeogo, J., Ye, M., . . Jahn, A. (2012). The impact of targeted subsidies for facility-based delivery on access to care and equity - evidence from a populationbased study in rural Burkina Faso. J Public Health Policy, 33(4), 439-453. doi:10.1057/jphp.2012.27

De Brouwere, V., Richard, F., \& Witter, S. (2010). Access to maternal and perinatal health services: lessons from successful and less successful examples of improving access to safe delivery and care of the newborn. Trop Med Int Health, 15(8), 901-909. doi:10.1111/j.1365-3156.2010.02558.x

Decker, M., \& Constantine, N. A. (2011). Factors associated with contraceptive use in Angola. Afr J Reprod Health, 15(4), 68-77.

Demery, L. (2000). In Benefits incidence: a practitioner's guide. Washington, D.C.: Poverty and Social Development Group, Africa Region, World Bank.

Demographic Health Survey. (2016). The DHS Program - Demographic and Health Survey (DHS). Retrieved from http://dhsprogram.com/Who-We-Are/About-Us.cfm

DerSimonian, R., \& Laird, N. (1986). Meta-analysis in clinical trials. Control Clin Trials, 7(3), 177-188. doi:10.1016/0197-2456(86)90046-2

Demographic Health Survey. (2018). DHS Methodology. Retrieved from https://dhsprogram.com/What-We- 


\section{Do/Survey-Types/DHS-Methodology.cfm}

Dixon, J., Tenkorang, E. Y., \& Luginaah, I. (2013). Ghana's National Health Insurance Scheme: a national level investigation of members' perceptions of service provision. BMC Int Health Hum Rights, 13(1), 35. doi:10.1186/1472-698X-13-35

Do, M., Soelaeman, R., \& Hotchkiss, D. R. (2015). Explaining inequity in the use of institutional delivery services in selected countries. Matern Child Health J, 19(4), 755-763. doi:10.1007/s10995-014-1561-5

Donabedian, A. (1973). Aspects of medical care administration: specifying requirements for health care: Harvard University Press.

Dong, H., Kouyate, B., Cairns, J., \& Sauerborn, R. (2005). Inequality in willingness-to-pay for community-based health insurance. Health Policy, 72(2), 149-156. doi:10.1016/j.healthpol.2004.02.014

Edu, B. C., Agan, T. U., Monjok, E., \& Makowiecka, K. (2017). Effect of Free Maternal Health Care Program on Health-seeking Behaviour of Women during Pregnancy, Intra-partum and Postpartum Periods in Cross River State of Nigeria: A Mixed Method Study. Open Access Maced J Med Sci, 5(3), 370-382. doi:10.3889/ oamjms.2017.075

Effective Public Health Practice Project. (1998). Quality assessment tool for quantitative studies. In (Vol. 2018): Effective Public Health Practice Project Hamilton, ON.

Egger, M., Davey Smith, G., Schneider, M., \& Minder, C. (1997). Bias in meta-analysis detected by a simple, graphical test. BMJ, 315(7109), 629-634. doi:10.1136/bmj.315.7109.629

Ekwempu, C. C., Maine, D., Olorukoba, M. B., Essien, E. S., \& Kisseka, M. N. (1990). Structural adjustment and health in Africa. Lancet, 336(8706), 56-57. doi:10.1016/0140-6736(90)91573-s

El-Khoury, M., Hatt, L., \& Gandaho, T. (2012). User fee exemptions and equity in access to caesarean sections: an analysis of patient survey data in Mali. Int J Equity Health, 11(1), 49. doi:10.1186/1475-9276-11-49

Ensor, T., \& Cooper, S. (2004). Overcoming barriers to health service access: influencing the demand side. Health Policy Plan, 19(2), 69-79. doi:10.1093/heapol/czh009

Exley, J., Pitchforth, E., Okeke, E., Glick, P., Abubakar, I. S., Chari, A., . . Onwujekwe, O. (2016). Persistent barriers to care; a qualitative study to understand women's experiences in areas served by the midwives service scheme in Nigeria. BMC Pregnancy Childbirth, 16(1), 232. doi:10.1186/s12884-016-1026-5

Ezeh, A., Bankole, A., Cleland, J., Garcia-Moreno, C., Temmerman, M., \& Ziraba, A. K. (2016). Burden of Reproductive III Health. In R. E. Black, R. Laxminarayan, M. Temmerman, \& N. Walker (Eds.), Reproductive, Maternal, Newborn, and Child Health: Disease Control Priorities, Third Edition (Volume 2). Washington (DC).

Fabamwo, A., \& Okonofua, F. (2010). An assessment of policies and programs for reducing maternal mortality in Lagos State, Nigeria. African Journal of Reproductive Health, 14(3), 55-63.

Fabic, M. S., Choi, Y., Bongaarts, J., Darroch, J. E., Ross, J. A., Stover, J., . . Starbird, E. (2015). Meeting demand for family planning within a generation: the post-2015 agenda. Lancet, 385(9981), 1928-1931. doi:10.1016/ S0140-6736(14)61055-2

Fagbamigbe, A. F., \& Idemudia, E. S. (2015). Barriers to antenatal care use in Nigeria: evidences from non-users and implications for maternal health programming. BMC Pregnancy Childbirth, 15(1), 95. doi:10.1186/ 
s12884-015-0527-y

Fagbamigbe, A. F., \& Idemudia, E. S. (2017). Wealth and antenatal care utilization in Nigeria: Policy implications. Health Care Women Int, 38(1), 17-37. doi:10.1080/07399332.2016.1225743

Fairlie, R. (2006). An extension of the Blinder-Oaxaca decomposition technique to logit and probit models.

Fairlie, R. W. (1999). The absence of the African-American owned business: An analysis of the dynamics of selfemployment. Journal of Labor Economics, 17(1), 80-108. doi:Doi 10.1086/209914

Fakunle, B., Okunlola, M. A., Fajola, A., Ottih, U., \& Ilesanmi, A. O. (2014). Community health insurance as a catalyst for uptake of family planning and reproductive health services: the Obio Cottage Hospital experience. J Obstet Gynaecol, 34(6), 501-503. doi:10.3109/01443615.2014.902044

Fichera, E., Gray, E., \& Sutton, M. (2016). How do individuals' health behaviours respond to an increase in the supply of health care? Evidence from a natural experiment. Soc Sci Med, 159, 170-179. doi:10.1016/j. socscimed.2016.05.005

Filippi, V., Ronsmans, C., Campbell, O. M., Graham, W. J., Mills, A., Borghi, J., . . Osrin, D. (2006). Maternal health in poor countries: the broader context and a call for action. Lancet, 368(9546), 1535-1541. doi:10.1016/ S0140-6736(06)69384-7

Filmer, D., \& Pritchett, L. H. (2001). Estimating wealth effects without expenditure data--or tears: an application to educational enrollments in states of India. Demography, 38(1), 115-132. doi:10.1353/dem.2001.0003

Finfgeld-Connett, D. (2010). Generalizability and transferability of meta-synthesis research findings. J Adv Nurs, 66(2), 246-254. doi:10.1111/j.1365-2648.2009.05250.x

Fleurbaey, M., \& Schokkaert, E. (2009). Unfair inequalities in health and health care. J Health Econ, 28(1), 73-90. doi:10.1016/j.jhealeco.2008.07.016

Federal Ministry of Health. (2001). National reproductive health policy and strategy: to achieve quality reproductive and sexual health for all Nigerians [Press release]

Federal Ministry of Health. (2009). National Reproductive Health Policy and Strategy to achieve quality Sexual and Reproductive and Sexual Health for all Nigerians. Abuja.

Federal Ministry of Health. (2012). National Health Insurance Scheme Operational Guidelines. Revised. Abuja, Nigeria.

Fortin, N., Lemieux, T., \& Firpo, S. (2011). Chapter 1 - Decomposition Methods in Economics. In O. Ashenfelter \& D. Card (Eds.), Handbook of Labor Economics (Vol. 4, pp. 1-102): Elsevier

Frenk, J. (1985). [Concept and measurement of accessibility]. Salud Publica Mex, 27(5), 438-453.

Gage, A. J., Ilombu, O., \& Akinyemi, A. I. (2016). Service readiness, health facility management practices, and delivery care utilization in five states of Nigeria: a cross-sectional analysis. BMC Pregnancy Childbirth, 16(1), 297. doi:10.1186/s12884-016-1097-3

Gakidou, E., \& Vayena, E. (2007). Use of modern contraception by the poor is falling behind. PLoS Med, 4(2), e31. doi:10.1371/journal.pmed.0040031

Galadanci, H., Idris, S., Sadauki, H., \& Yakasai, I. (2010). Programs and policies for reducing maternal mortality in Kano State, Nigeria: a review. African Journal of Reproductive Health, 14(3), 31-36.

Ganle, J. K., Parker, M., Fitzpatrick, R., \& Otupiri, E. (2014). Inequities in accessibility to and utilisation of 
maternal health services in Ghana after user-fee exemption: a descriptive study. Int J Equity Health, 13, 89. doi:10.1186/s12939-014-0089-z

Garenne, M. (2018). Family Planning and Fertility Decline in Africa: From 1950 to 2010. In Family Planning: IntechOpen.

Gavin, L., Moskosky, S., Carter, M., Curtis, K., Glass, E., Godfrey, E., .. . Prevention. (2014). Providing quality family planning services: Recommendations of CDC and the U.S. Office of Population Affairs. MMWR Recomm Rep, 63(RR-04), 1-54.

Gentes, E. L., \& Ruscio, A. M. (2011). A meta-analysis of the relation of intolerance of uncertainty to symptoms of generalized anxiety disorder, major depressive disorder, and obsessive-compulsive disorder. Clin Psychol Rev, 31(6), 923-933. doi:10.1016/j.cpr.2011.05.001

Ghana Statistical Service, Ghana Health Service, \& ICF International. (2014). Ghana Demographic and Health Survey. Ghana Demographic and Health Survey 2014. In. Retrieved from https://dhsprogram.com/pubs/ pdf/FR307/FR307.pdf

Ghana Health Service. (2017). Background: Ghana Health Service. Retrieved from http://www. ghanahealthservice.org/ghs-subcategory.php?cid=28scid=42\#

Gillespie, D. (2007). Contraceptive use and the poor: a matter of choice? PLoS Med, 4(2), e49. doi:10.1371/ journal.pmed.0040049

Gilson, L., Kalyalya, D., Kuchler, F., Lake, S., Oranga, H., \& Ouendo, M. (2001). Strategies for promoting equity: experience with community financing in three African countries. Health Policy, 58(1), 37-67. doi:10.1016/ s0168-8510(01)00153-1

Gilson, L., \& Mclntyre, D. (2005). Removing user fees for primary care in Africa: the need for careful action. BMJ, 331(7519), 762-765. doi:10.1136/bmj.331.7519.762

Glasier, A., Gulmezoglu, A. M., Schmid, G. P., Moreno, C. G., \& Van Look, P. F. (2006). Sexual and reproductive health: a matter of life and death. Lancet, 368(9547), 1595-1607. doi:10.1016/S0140-6736(06)69478-6

Global Health Observatory. (2020). Key Country Indicators. Retrieved from http://apps.who.int/gho/data/node. $\underline{\mathrm{CCO}}$

Goli, S., Doshi, R., \& Perianayagam, A. (2013). Pathways of economic inequalities in maternal and child health in urban India: a decomposition analysis. PLoS One, 8(3), e58573. doi:10.1371/journal.pone.0058573

Goli, S., Nawal, D., Rammohan, A., Sekher, T. V., \& Singh, D. (2018). Decomposing the Socioeconomic Inequality in Utilization of Maternal Health Care Services in Selected Countries of South Asia and Sub-Saharan Africa. Journal of Biosocial Science, 50(6), 749-769. doi:10.1017/S0021932017000530

Ghana Statistical Service, Ghana Health Service, \& ICF International. (2009). Ghana Demographic and Health Survey 2008. Retrieved from Accra:

Ghana Statistical Service, Ghana Health Service, \& ICF International. (2015). Ghana Demographic and Health Survey 2014. Rockville, Maryland, USA: GSS, GHS, and ICF International.

Guengant, J. (2012). Comment Beneficier du Dividende Demographique? La Demographie au Centre des Trajectoires de Developpement dans les Pays de I'UEMOA. Analyse pays Niger.

Gulliford, M., Figueroa-Munoz, J., Morgan, M., Hughes, D., Gibson, B., Beech, R., \& Hudson, M. (2002). What does 
'access to health care' mean? J Health Serv Res Policy, 7(3), 186-188. doi:10.1258/135581902760082517 Gupta, N., Katende, C., \& Bessinger, R. (2003). Associations of mass media exposure with family planning attitudes and practices in Uganda. Stud Fam Plann, 34(1), 19-31. doi:10.1111/j.1728-4465.2003.00019.x

Gwatkin, D. R., Bhuiya, A., \& Victora, C. G. (2004). Making health systems more equitable. Lancet, 364(9441), 1273-1280. doi:10.1016/S0140-6736(04)17145-6

Gyimah, S. O., Takyi, B. K., \& Addai, I. (2006). Challenges to the reproductive-health needs of African women: on religion and maternal health utilization in Ghana. Soc Sci Med, 62(12), 2930-2944. doi:10.1016/j. socscimed.2005.11.034

Hatt, L. E., Makinen, M., Madhavan, S., \& Conlon, C. M. (2013). Effects of User Fee Exemptions on the Provision and Use of Maternal Health Services: A Review of Literature. Journal of Health Population and Nutrition, $31(4), 67-80$

Hercot, D., Meessen, B., Ridde, V., \& Gilson, L. (2011). Removing user fees for health services in low-income countries: a multi-country review framework for assessing the process of policy change. Health Policy Plan, 26 Suppl 2, ii5-15. doi:10.1093/heapol/czr063

Higgins, J. P., Thompson, S. G., Deeks, J. J., \& Altman, D. G. (2003). Measuring inconsistency in meta-analyses. BMJ, 327(7414), 557-560. doi:10.1136/bmj.327.7414.557

Honda, A., Randaoharison, P. G., \& Matsui, M. (2011). Affordability of emergency obstetric and neonatal care at public hospitals in Madagascar. Reprod Health Matters, 19(37), 10-20. doi:10.1016/S0968-8080(11)375593

Hosseinpoor, A. R., Victora, C. G., Bergen, N., Barros, A. J., \& Boerma, T. (2011). Towards universal health coverage: the role of within-country wealth-related inequality in 28 countries in sub-Saharan Africa. Bull World Health Organ, 89(12), 881-890. doi:10.2471/BLT.11.087536

Hotchkiss, D. R., Godha, D., \& Do, M. (2011). Effect of an expansion in private sector provision of contraceptive supplies on horizontal inequity in modern contraceptive use: evidence from Africa and Asia. Int J Equity Health, 10(1), 33. doi:10.1186/1475-9276-10-33

Hounton, S., Barros, A. J., Amouzou, A., Shiferaw, S., Maiga, A., Akinyemi, A., . . Koroma, D. (2015). Patterns and trends of contraceptive use among sexually active adolescents in Burkina Faso, Ethiopia, and Nigeria: evidence from cross-sectional studies. Glob Health Action, 8, 29737. doi:10.3402/gha.v8.29737

Houweling, T. A., Kunst, A. E., Huisman, M., \& Mackenbach, J. P. (2007). Using relative and absolute measures for monitoring health inequalities: experiences from cross-national analyses on maternal and child health. Int J Equity Health, 6, 15. doi:10.1186/1475-9276-6-15

Hsu, J., Berman, P., \& Mills, A. (2013). Reproductive health priorities: evidence from a resource tracking analysis of official development assistance in 2009 and 2010. Lancet, 381(9879), 1772-1782. doi:10.1016/S0140$6736(13) 60762-X$

Ibiwoye, A., \& Adeleke, I. A. (2008). Does National Health Insurance Promote Access to Quality Health Care? Evidence from Nigeria. The Geneva Papers on Risk and Insurance - Issues and Practice, 33(2), 219-233. doi:10.1057/gpp.2008.6

International Conference on Population Development. (2014). Programme of Action of the International 
Conference on Population Development. Retrieved from https://www.unfpa.org/publications/ international-conference-population-and-development-programme-action

Idowu, A., Olowookere, S. A., Abiola, O. O., Akinwumi, A. F., \& Adegbenro, C. (2017). Determinants of Skilled Care Utilization among Pregnant Women Residents in an Urban Community in Kwara State, Northcentral Nigeria. Ethiop J Health Sci, 27(3), 291-298. doi:10.4314/ejhs.v27i3.11

Ijadunola, K. T., Ijadunola, M. Y., Esimai, O. A., \& Abiona, T. C. (2010). New paradigm old thinking: The case for emergency obstetric care in the prevention of maternal mortality in Nigeria. BMC Womens Health, $10,6$. doi:10.1186/1472-6874-10-6

Institut National de la Statistique et de la Démographie - INSD/Burkina Faso, \& ICF International. (2012). Burkina Faso Enquête Démographique et de Santé et à Indicateurs Multiples (EDSBF-MICS IV) 2010. Retrieved from Calverton, Maryland, USA: http://dhsprogram.com/pubs/pdf/FR256/FR256.pdf

Institut National de la Statistique/Niger, UNICEF/Niger, \& ICF International. (2013). Enquête Démographique et de Santé dans les Zones d'Intervention du Programme de Cooperation de I'UNICEF au Niger, 2012. Retrieved from Rockville, Maryland, USA: http://dhsprogram.com/pubs/pdf/FR285/FR285.pdf

Irwin, A., Valentine, N., Brown, C., Loewenson, R., Solar, O., Brown, H., . . . Vega, J. (2006). The commission on social determinants of health: tackling the social roots of health inequities. PLoS Med, 3(6), e106. doi:10.1371/journal.pmed.0030106

Jallow, I. K., Chou, Y. J., Liu, T. L., \& Huang, N. (2012). Women's perception of antenatal care services in public and private clinics in the Gambia. Int J Qual Health Care, 24(6), 595-600. doi:10.1093/intqhc/mzs033

Johnson, F. A., Frempong-Ainguah, F., \& Padmadas, S. S. (2016). Two decades of maternity care fee exemption policies in Ghana: have they benefited the poor? Health Policy Plan, 31(1), 46-55. doi:10.1093/heapol/ CZv017

Johnson, F. A., Padmadas, S. S., \& Brown, J. J. (2009). On the spatial inequalities of institutional versus home births in Ghana: a multilevel analysis. J Community Health, 34(1), 64-72. doi:10.1007/s10900-008-9120-x

Jonas, K., Crutzen, R., van den Borne, B., \& Reddy, P. (2017). Healthcare workers' behaviors and personal determinants associated with providing adequate sexual and reproductive healthcare services in subSaharan Africa: a systematic review. BMC Pregnancy Childbirth, 17(1), 86. doi:10.1186/s12884-017-1268-x Kabeer, N. (2012). Women's economic empowerment and inclusive growth: labour markets and enterprise development. International Development Research Centre, 44(10), 1-70.

Kabir, M., Iliyasu, Z., Abubakar, I. S., \& Asani, A. (2005). Determinants of utilization of antenatal care services in Kumbotso Village, northern Nigeria. Trop Doct, 35(2), 110-111. doi:10.1258/0049475054036814

Kakwani, N., Wagstaff, A., \& vanDoorslaer, E. (1997). Socioeconomic inequalities in health: Measurement, computation, and statistical inference. Journal of Econometrics, 77(1), 87-103. doi:Doi 10.1016/S03044076(96)01807-6

Karim, A. M., Tamire, A., Medhanyie, A. A., \& Betemariam, W. (2015). Changes in equity of maternal, newborn, and child health care practices in 115 districts of rural Ethiopia: implications for the health extension program. BMC Pregnancy Childbirth, 15, 238. doi:10.1186/s12884-015-0668-z

Kendall, T., \& Langer, A. (2015). Critical maternal health knowledge gaps in low- and middle-income countries 
for the post-2015 era. Reprod Health, 12(1), 55. doi:10.1186/s12978-015-0044-5

Kerber, K. J., de Graft-Johnson, J. E., Bhutta, Z. A., Okong, P., Starrs, A., \& Lawn, J. E. (2007). Continuum of care for maternal, newborn, and child health: from slogan to service delivery. Lancet, 370(9595), 1358-1369. doi:10.1016/S0140-6736(07)61578-5

Knapp, G., \& Hartung, J. (2003). Improved tests for a random effects meta-regression with a single covariate. Stat Med, 22(17), 2693-2710. doi:10.1002/sim.1482

Koblinsky, M., Matthews, Z., Hussein, J., Mavalankar, D., Mridha, M. K., Anwar, I., . . Lancet Maternal Survival Series steering, g. (2006). Going to scale with professional skilled care. Lancet, 368(9544), 1377-1386. doi:10.1016/S0140-6736(06)69382-3

Koblinsky, M. A., Campbell, O., \& Heichelheim, J. (1999). Organizing delivery care: what works for safe motherhood? Bull World Health Organ, 77(5), 399-406.

Koduah, A., Agyepong, I. A., \& van Dijk, H. (2016). 'The one with the purse makes policy': Power, problem definition, framing and maternal health policies and programmes evolution in national level institutionalised policy making processes in Ghana. Soc Sci Med, 167, 79-87. doi:10.1016/j.socscimed.2016.08.051

Koduah, A., Agyepong, I. A., \& van Dijk, H. (2018). Towards an explanatory framework for national level maternal health policy agenda item evolution in Ghana: an embedded case study. Health Res Policy Syst, 16(1), 76. doi:10.1186/s12961-018-0354-5

Koduah, A., van Dijk, H., \& Agyepong, I. A. (2015). The role of policy actors and contextual factors in policy agenda setting and formulation: maternal fee exemption policies in Ghana over four and a half decades. Health Res Policy Syst, 13(1), 27. doi:10.1186/s12961-015-0016-9

Koster, W., Ondoa, P., Sarr, A. M., Sow, A. I., Schultsz, C., Sakande, J., . . . Pool, R. (2016). Barriers to uptake of antenatal maternal screening tests in Senegal. SSM Popul Health, 2, 784-792. doi:10.1016/j. ssmph.2016.10.003

Krenn, S., Cobb, L., Babalola, S., Odeku, M., \& Kusemiju, B. (2014). Using behavior change communication to lead a comprehensive family planning program: the Nigerian Urban Reproductive Health Initiative. Glob Health Sci Pract, 2(4), 427-443. doi:10.9745/GHSP-D-14-00009

Kwankye, S. O., \& Augustt, E. (2013). Media exposure and reproductive health behaviour among young females in Ghana. African Population Studies, 22(2).

Lassi, Z. S., Salam, R. A., Das, J. K., \& Bhutta, Z. A. (2014). Essential interventions for maternal, newborn and child health: background and methodology. Reprod Health, 11 Suppl 1(1), S1. doi:10.1186/1742-4755-11-S1-S1

Levesque, J. F., Harris, M. F., \& Russell, G. (2013). Patient-centred access to health care: conceptualising access at the interface of health systems and populations. Int J Equity Health, 12(1), 18. doi:10.1186/1475-9276$12-18$

Liao, P.-A., Chang, H.-H., Wang, J.-H., \& Sun, L.-C. (2016). What are the determinants of rural-urban digital inequality among schoolchildren in Taiwan? Insights from Blinder-Oaxaca decomposition. Computers $\&$ Education, 95, 123-133. doi:10.1016/j.compedu.2016.01.002

Liu, X., Gao, W., \& Yan, H. (2014). Measuring and decomposing the inequality of maternal health services utilization in western rural China. BMC Health Serv Res, 14(1), 102. doi:10.1186/1472-6963-14-102 
Makinen, M., Waters, H., Rauch, M., Almagambetova, N., Bitran, R., Gilson, L., . . Ram, S. (2000). Inequalities in health care use and expenditures: empirical data from eight developing countries and countries in transition. Bulletin of the World Health Organization, 78(1), 55-65.

Makoka, D. (2009). Towards an understanding of regional disparities in social inequities in maternal health in Malawi. Afr Health Sci, 9(4), 234-241.

Mangham-Jefferies, L., Pitt, C., Cousens, S., Mills, A., \& Schellenberg, J. (2014). Cost-effectiveness of strategies to improve the utilization and provision of maternal and newborn health care in low-income and lowermiddle-income countries: a systematic review. BMC Pregnancy Childbirth, 14(1), 243. doi:10.1186/14712393-14-243

Marmot, M. (2005). Social determinants of health inequalities. Lancet, 365(9464), 1099-1104. doi:10.1016/ S0140-6736(05)71146-6

Mayhew, S. H. (2000). Integration of STI services into FP/MCH services: health service and social contexts in rural Ghana. Reprod Health Matters, 8(16), 112-124. doi:10.1016/s0968-8080(00)90193-9

McCollum, R., Gomez, W., Theobald, S., \& Taegtmeyer, M. (2016). How equitable are community health worker programmes and which programme features influence equity of community health worker services? A systematic review. BMC Public Health, 16(1), 419

McKinnon, B., Harper, S., \& Kaufman, J. S. (2015). Who benefits from removing user fees for facility-based delivery services? Evidence on socioeconomic differences from Ghana, Senegal and Sierra Leone. Soc Sci Med, 135, 117-123. doi:10.1016/j.socscimed.2015.05.003

Medhanyie, A., Spigt, M., Kifle, Y., Schaay, N., Sanders, D., Blanco, R., . . Berhane, Y. (2012). The role of health extension workers in improving utilization of maternal health services in rural areas in Ethiopia: a cross sectional study. BMC Health Serv Res, 12(1), 352. doi:10.1186/1472-6963-12-352

Meessen, B., Hercot, D., Noirhomme, M., Ridde, V., Tibouti, A., Bicaba, A., . . Gilson, L. (2009). Removing user fees in the health sector in low-income countries: a multi-country review. Retrieved from OAlster database. United Nations Children's Fund (UNICEF).

Mekonnen, Y., \& Mekonnen, A. (2002). Utilization of maternal health care services in Ethiopia. Retrieved from Calverton, Maryland, USA: http://dhsprogram.com/pubs/pdf/FA38/FA38.pdf

Mellor, J. M., \& Milyo, J. D. (2001). Income Inequality and Health. Journal of Policy Analysis and Management, 20(1), 151-155

Mensah, J., Oppong, J. R., \& Schmidt, C. M. (2010). Ghana's National Health Insurance Scheme in the context of the health MDGs: an empirical evaluation using propensity score matching. Health Econ, 19 Suppl, 95-106. doi:10.1002/hec.1633

Mezmur, M., Navaneetham, K., Letamo, G., \& Bariagaber, H. (2017). Socioeconomic inequalities in the uptake of maternal healthcare services in Ethiopia. BMC Health Serv Res, 17(1), 367. doi:10.1186/s12913-017-2298-9

Mills, A., Ataguba, J. E., Akazili, J., Borghi, J., Garshong, B., Makawia, S., . . Mclntyre, D. (2012). Equity in financing and use of health care in Ghana, South Africa, and Tanzania: implications for paths to universal coverage. Lancet, 380(9837), 126-133. doi:10.1016/S0140-6736(12)60357-2

Ministry of Health Ghana. (2008). Independent review of health sector programme of work 2007. Ministry of 
Health Accra, Ghana.

Mirzoev, T., Etiaba, E., Ebenso, B., Uzochukwu, B., Manzano, A., Onwujekwe, O., . . Ensor, T. (2016). Study protocol: realist evaluation of effectiveness and sustainability of a community health workers programme in improving maternal and child health in Nigeria. Implement Sci, 11(1), 83. doi:10.1186/s13012-0160443-1

Mohammed, S., \& Dong, H. (2012). Tackling Millennium Development Goals (MDGs) 4 and 5: The National Health Insurance Scheme (NHIS) Approach in Nigeria. J Public Health Afr, 3(1), e9. doi:10.4081/jphia.2012. e9

Moher, D., Liberati, A., Tetzlaff, J., \& Altman, D. G. (2009). Preferred reporting items for systematic reviews and meta-analyses: the PRISMA statement. Ann Intern Med, 151(4), 264-269, W264. doi:10.7326/0003-4819151-4-200908180-00135

Moller, A. B., Patten, J. H., Hanson, C., Morgan, A., Say, L., Diaz, T., \& Moran, A. C. (2019). Monitoring maternal and newborn health outcomes globally: a brief history of key events and initiatives. Trop Med Int Health, 24(12), 1342-1368. doi:10.1111/tmi.13313

Mrisho, M., Obrist, B., Schellenberg, J. A., Haws, R. A., Mushi, A. K., Mshinda, H., . . Schellenberg, D. (2009). The use of antenatal and postnatal care: perspectives and experiences of women and health care providers in rural southern Tanzania. BMC Pregnancy Childbirth, 9, 10. doi:10.1186/1471-2393-9-10

Mutangadura, G., Gauci, A., Armah, B., Woldemariam, E., Ayalew, D., \& Egu, B. (2007). Health inequities in selected African countries: Review of evidence and policy implications. Economic Commission for Africa.

Mwase, T., Brenner, S., Mazalale, J., Lohmann, J., Hamadou, S., Somda, S. M. A., . . De Allegri, M. (2018). Inequities and their determinants in coverage of maternal health services in Burkina Faso. Int J Equity Health, 17(1), 58. doi:10.1186/s12939-018-0770-8

Ngandu, N. K., Van Malderen, C., Goga, A., \& Speybroeck, N. (2017). Wealth-related inequality in early uptake of HIV testing among pregnant women: an analysis of data from a national cross-sectional survey, South Africa. BMJ Open, 7(7), e013362. doi:10.1136/bmjopen-2016-013362

Nghargbu, R., \& Olaniyan, O. (2017). Inequity in Maternal and Child Health Care Utilization in Nigeria. African Development Review, 29(4), 630-647. doi:10.1111/1467-8268.12301

Nguyen, H. T., Rajkotia, Y., \& Wang, H. (2011). The financial protection effect of Ghana National Health Insurance Scheme: evidence from a study in two rural districts. Int J Equity Health, 10(1), 4. doi:10.1186/1475-9276$10-4$

National Health Insurance Authority. MoH PPME Health Sector Indicator Database. Retrieved from http://www. moh.gov.gh/wp-content/uploads/

National Health Insurance Authority. (2012). NHIS-MDG/Maternal and Child Health (MCH) Project. In: NHIS Abuja.

National Population Commission. (2009). Nigeria demographic and health survey 2008. Abuja: National Population Commission, 630

National Population Commission \& ICF International. (2014). Nigeria Demographic and Health Survey 2013. Retrieved from Abuja, Nigeria: http://dhsprogram.com/pubs/pdf/FR293/FR293.pdf 
National Primary Health Care Development Agency (2018). Nigeria Midwives Service Scheme. Retrieved from http://www.who.int/workforcealliance/forum/2011/hrhawardscs26/en/

Ngome, E., \& Odimegwu, C. (2014). The social context of adolescent women's use of modern contraceptives in Zimbabwe: a multilevel analysis. Reprod Health, 11, 64. doi:10.1186/1742-4755-11-64

Nyonator, F., \& Kutzin, J. (1999). Health for some? The effects of user fees in the Volta Region of Ghana. Health Policy Plan, 14(4), 329-341. doi:10.1093/heapol/14.4.329

Nyonator, F. K., Awoonor-Williams, J. K., Phillips, J. F., Jones, T. C., \& Miller, R. A. (2005). The Ghana communitybased health planning and services initiative for scaling up service delivery innovation. Health Policy Plan, 20(1), 25-34. doi:10.1093/heapol/czi003

O'Donnell, O., O'Neill, S., Van Ourti, T., \& Walsh, B. (2016). conindex: Estimation of concentration indices. Stata J, 16(1), 112-138.

O'Donnell, O., Van Doorslaer, E., Wagstaff, A., \& Lindelow, M. (2008). Analyzing health equity using household survey data: a guide to techniques and their implementation: Washington, DC: World Bank.

O’Neill, J., Tabish, H., Welch, V., Petticrew, M., Pottie, K., Clarke, M., ... Tugwell, P. (2014). Applying an equity lens to interventions: using PROGRESS ensures consideration of socially stratifying factors to illuminate inequities in health. J Clin Epidemiol, 67(1), 56-64. doi:10.1016/j.jclinepi.2013.08.005

Obiyan, M. O., \& Kumar, A. (2015). Socioeconomic Inequalities in the Use of Maternal Health Care Services in Nigeria. SAGE Open, 5(4), 2158244015614070. doi:10.1177/2158244015614070

Ochako, R., Fotso, J. C., Ikamari, L., \& Khasakhala, A. (2011). Utilization of maternal health services among young women in Kenya: insights from the Kenya Demographic and Health Survey, 2003. BMC Pregnancy Childbirth, 11(1), 1. doi:10.1186/1471-2393-11-1

Odeyemi, I. A., \& Nixon, J. (2013). Assessing equity in health care through the national health insurance schemes of Nigeria and Ghana: a review-based comparative analysis. Int J Equity Health, 12(1), 9. doi:10.1186/14759276-12-9

Ogbuabor, D. C., Onwujekwe, O. E., \& Ezumah, N. (2019). Muddling through policymaking: A complex adaptive systems perspective on policy changes in a free maternal and child healthcare program in Enugu State, Nigeria. Niger J Clin Pract, 22(5), 682-691. doi:10.4103/njcp.njcp_379_18

Okafor, I., Obi, S., \& Ugwu, E. (2010). Impact of Free Maternal and Child Healthcare programme on maternal and neonatal healthcare outcome in Enugu State of Nigeria. Niger J Med, 20.

Okebukola, P. O., \& Brieger, W. R. (2016). Providing Universal Health Insurance Coverage in Nigeria. Int Q Community Health Educ, 36(4), 241-246. doi:10.1177/0272684X16657451

Okeke, E. N., Glick, P., \& Abubakar Isa, S. (2015). The Better Obstetrics in Rural Nigeria (BORN) Study: An Impact Evaluation of the Nigerian Midwives Service Scheme: Rand Corporation.

Okonofua, F., Lambo, E., Okeibunor, J., \& Agholor, K. (2011). Advocacy for free maternal and child health care in Nigeria--Results and outcomes. Health Policy, 99(2), 131-138. doi:10.1016/j.healthpol.2010.07.013

Okonofua, F. E., Ntoimo, L. F. C., \& Ogu, R. N. (2018). Women's perceptions of reasons for maternal deaths: Implications for policies and programs for preventing maternal deaths in low-income countries. Health Care Women Int, 39(1), 95-109. doi:10.1080/07399332.2017.1365868 
Okpani, A. I., \& Abimbola, S. (2015). Operationalizing universal health coverage in Nigeria through social health insurance. Niger Med J, 56(5), 305-310. doi:10.4103/0300-1652.170382

Okpani, A. I., \& Abimbola, S. (2016). The midwives service scheme: a qualitative comparison of contextual determinants of the performance of two states in central Nigeria. Glob Health Res Policy, 1(1), 16. doi:10.1186/s41256-016-0017-4

Olakunde, B. (2012). Public health care financing in Nigeria: Which way forward? Annals of Nigerian Medicine, 6(1), 4. doi:10.4103/0331-3131.100199

Olaniran, A., Madaj, B., Bar-Zev, S., \& van den Broek, N. (2019). The roles of community health workers who provide maternal and newborn health services: case studies from Africa and Asia. BMJ Glob Health, 4(4), e001388. doi:10.1136/bmjgh-2019-001388

Omeje, J. C., Oshi, S. N., \& Oshi, D. C. (2011). Does possession of assets increase women's participation in reproductive decision-making? Perceptions of Nigerian women. Journal of Biosocial Science, 43(1), 101111. doi:10.1017/S0021932010000520

Onah, H. E., Ikeako, L. C., \& Iloabachie, G. C. (2006). Factors associated with the use of maternity services in Enugu, southeastern Nigeria. Soc Sci Med, 63(7), 1870-1878. doi:10.1016/j.socscimed.2006.04.019

Onarheim, K. H., Taddesse, M., Norheim, O. F., Abdullah, M., \& Miljeteig, I. (2015). Towards universal health coverage for reproductive health services in Ethiopia: two policy recommendations. Int J Equity Health, 14, 86. doi:10.1186/s12939-015-0218-3

Onwuhafua, P. I., Kantiok, C., Olafimihan, O., \& Shittu, O. S. (2005). Knowledge, attitude and practice of family planning amongst community health extension workers in Kaduna State, Nigeria. J Obstet Gynaecol, 25(5), 494-499. doi:10.1080/01443610500171201

Onwujekwe, O., Obi, F., \& Uzochukwu, B. (2016). Assessment of the NHIS-MDG free Maternal and Child Health program and the prospects for program re-activation/scale-up using the Basic Health Care Provision Fund in Nigeria.

Onwujekwe, O., Onoka, C., Uzochukwu, B., \& Hanson, K. (2011). Constraints to universal coverage: inequities in health service use and expenditures for different health conditions and providers. Int J Equity Health, 10, 50. doi:10.1186/1475-9276-10-50

Onwujekwe, O., Onoka, C., Uzochukwu, B., Okoli, C., Obikeze, E., \& Eze, S. (2009). Is community-based health insurance an equitable strategy for paying for healthcare? Experiences from southeast Nigeria. Health Policy, 92(1), 96-102. doi:10.1016/j.healthpol.2009.02.007

Onwuzurike, B. K., \& Uzochukwu, B. S. (2001). Knowledge, attitude and practice of family planning amongst women in a high density low income urban of Enugu, Nigeria. Afr J Reprod Health, 5(2), 83-89.

Orbell, S., Schneider, H., Esbitt, S., Gonzalez, J. S., Gonzalez, J. S., Shreck, E., . . Turner, J. R. (2013). Health Care Utilization. In M. D. Gellman \& J. R. Turner (Eds.), Encyclopedia of Behavioral Medicine (pp. 909-910). New York, NY: Springer New York.

Overbosch, G. B., Nsowah-Nuamah, N. N. N., van den Boom, G. J. M., \& Damnyag, L. (2004). Determinants of antenatal care use in Ghana. Journal of African Economies, 13(2), 277-301. doi:10.1093/jae/ejh008

Paina, L., \& Peters, D. H. (2012). Understanding pathways for scaling up health services through the lens of 
complex adaptive systems. Health Policy Plan, 27(5), 365-373. doi:10.1093/heapol/czr054

Pearson, L., Gandhi, M., Admasu, K., \& Keyes, E. B. (2011). User fees and maternity services in Ethiopia. Int J Gynaecol Obstet, 115(3), 310-315. doi:10.1016/j.ijgo.2011.09.007

Pell, C., Menaca, A., Were, F., Afrah, N. A., Chatio, S., Manda-Taylor, L., . . Pool, R. (2013). Factors affecting antenatal care attendance: results from qualitative studies in Ghana, Kenya and Malawi. PLoS One, 8(1), e53747. doi:10.1371/journal.pone.0053747

Penchansky, R., \& Thomas, J. W. (1981). The concept of access: definition and relationship to consumer satisfaction. Med Care, 19(2), 127-140. doi:10.1097/00005650-198102000-00001

Perkins, M., Brazier, E., Themmen, E., Bassane, B., Diallo, D., Mutunga, A., . . Ngobola, O. (2009). Out-of-pocket costs for facility-based maternity care in three African countries. Health Policy Plan, 24(4), 289-300. doi:10.1093/heapol/czp013

Peters, D. H., Garg, A., Bloom, G., Walker, D. G., Brieger, W. R., \& Rahman, M. H. (2008). Poverty and access to health care in developing countries. Ann N Y Acad Sci, 1136(1), 161-171. doi:10.1196/annals.1425.011

Phillips, J. F., Bawah, A. A., \& Binka, F. N. (2005). Accelerating reproductive and child health program development: the Navrongo initiative in Ghana.

Ravit, M., Audibert, M., Ridde, V., De Loenzien, M., Schantz, C., \& Dumont, A. (2018). Do free caesarean section policies increase inequalities in Benin and Mali? Int J Equity Health, 17(1), 71. doi:10.1186/s12939-0180789-x

Reichenbach, L. (2002). The politics of priority setting for reproductive health: breast and cervical cancer in Ghana. Reprod Health Matters, 10(20), 47-58. doi:10.1016/s0968-8080(02)00093-9

Richard, F., Antony, M., Witter, S., Kelley, A., Sieleunou, I., Kafando, Y., \& Meessen, B. (2013). Fee exemption for maternal care in sub-Saharan Africa: a review of 11 countries and lessons for the region. Global Health Governance, 6(2), 52-72.

Richard, F., Witter, S., \& de Brouwere, V. (2010). Innovative approaches to reducing financial barriers to obstetric care in low-income countries. Am J Public Health, 100(10), 1845-1852. doi:10.2105/AJPH.2009.179689

Ridde, V., Agier, I., Jahn, A., Mueller, O., Tiendrebeogo, J., Ye, M., \& De Allegri, M. (2015). The impact of user fee removal policies on household out-of-pocket spending: evidence against the inverse equity hypothesis from a population based study in Burkina Faso. Eur J Health Econ, 16(1), 55-64. doi:10.1007/s10198-0130553-5

Ridde, V., \& Diarra, A. (2009). A process evaluation of user fees abolition for pregnant women and children under five years in two districts in Niger (West Africa). BMC Health Serv Res, 9(1), 89. doi:10.1186/14726963-9-89

Ridde, V., Kouanda, S., Bado, A., Bado, N., \& Haddad, S. (2012). Reducing the medical cost of deliveries in Burkina Faso is good for everyone, including the poor. PLoS One, 7(3), e33082. doi:10.1371/journal.pone.0033082

Ridde, V., \& Morestin, F. (2011). A scoping review of the literature on the abolition of user fees in health care services in Africa. Health Policy Plan, 26(1), 1-11. doi:10.1093/heapol/czq021

Rodney, A. M., \& Hill, P. S. (2014). Achieving equity within universal health coverage: a narrative review of progress and resources for measuring success. Int J Equity Health, 13, 72. doi:10.1186/s12939-014-0072- 
Rogo, K. O., Oucho, J., \& Mwalali, P. (2006). Maternal Mortality. In nd, D. T. Jamison, R. G. Feachem, M. W. Makgoba, E. R. Bos, F. K. Baingana, K. J. Hofman, \& K. O. Rogo (Eds.), Disease and Mortality in Sub-Saharan Africa. Washington (DC): The International Bank for Reconstruction and Development/The World Bank.

Romesburg, C. (2004). Cluster analysis for researchers: Lulu. com.

Rosenblad, A. (2009). Effect Sizes Based on Correlations. In Introduction to Meta区Analysis by Michael Borenstein, Larry V. Hedges, Julian PT Higgins, Hannah R. Rothstein: International Statistical Review.

Rosenfield, A., \& Maine, D. (1985). Maternal mortality--a neglected tragedy. Where is the M in MCH? Lancet, 2(8446), 83-85. doi:10.1016/s0140-6736(85)90188-6

Ross, J. (2015). Improved Reproductive Health Equity Between the Poor and the Rich: An Analysis of Trends in 46 Low- and Middle-Income Countries. Glob Health Sci Pract, 3(3), 419-445. doi:10.9745/GHSP-D-15-00124

Rossier, C., Muindi, K., Soura, A., Mberu, B., Lankoande, B., Kabiru, C., \& Millogo, R. (2014). Maternal health care utilization in Nairobi and Ouagadougou: evidence from HDSS. Glob Health Action, 7, 24351. doi:10.3402/ gha.v7.24351

Rudan, I., Kapiriri, L., Tomlinson, M., Balliet, M., Cohen, B., \& Chopra, M. (2010). Evidence-based priority setting for health care and research: tools to support policy in maternal, neonatal, and child health in Africa. PLOS Med, 7(7), e1000308. doi:10.1371/journal.pmed.1000308

Russell, S., \& Gilson, L. (1997). User fee policies to promote health service access for the poor: a wolf in sheep's clothing? Int J Health Serv, 27(2), 359-379. doi:10.2190/YHL2-FOEA-JW1M-DHEJ

Rutstein, S. O., \& Johnson, K. (2004). The DHS wealth index. DHS comparative reports no. 6. Calverton: ORC Macro.

Sahn, D., \& Bernier, R. (1995). Have structural adjustments led to health sector reform in Africa? Health Policy, 32(1-3), 193-214. doi:10.1016/0168-8510(95)00736-c

Samb, O. M., \& Ridde, V. (2018). The impact of free healthcare on women's capability: A qualitative study in rural Burkina Faso. Soc Sci Med, 197, 9-16. doi:10.1016/j.socscimed.2017.11.045

Schneider, H., \& Gilson, L. (2006). The impact of free maternal health care in South Africa. In M. Berer \& T. K. S. Ravindran (Eds.), Safe motherhood initiatives: critical issues. Oxford: Blackwell Science, for Reproductive Health Matters.

Schuetz, T. (2011). A concise guide to market research: the process, data and methods using IBM SPSS statistics. In: MARKET RESEARCH SOC 15 NORTHBURGH ST, LONDON EC1V 0JR, ENGLAND.

Shengelia, B., Murray, C. J., \& Adams, O. B. (2003). Beyond access and utilization: defining and measuring health system coverage. Health systems performance assessment: debates, methods and empiricism. Geneva: World Health Organization, 221-234.

Shiffman, J., \& Okonofua, F. E. (2007). The state of political priority for safe motherhood in Nigeria. BJOG, 114(2), 127-133. doi:10.1111/j.1471-0528.2006.01184.x

Shimamoto, K., \& Gipson, J. D. (2017). Examining the mechanisms by which women's status and empowerment affect skilled birth attendant use in Senegal: a structural equation modeling approach. BMC Pregnancy Childbirth, 17(Suppl 2), 341. doi:10.1186/s12884-017-1499-x 
Simkhada, B., Teijlingen, E. R., Porter, M., \& Simkhada, P. (2008). Factors affecting the utilization of antenatal care in developing countries: systematic review of the literature. J Adv Nurs, 61(3), 244-260. doi:10.1111/ j.1365-2648.2007.04532.x

Simon, G. E., Goldberg, D. P., Von Korff, M., \& Ustun, T. B. (2002). Understanding cross-national differences in depression prevalence. Psychol Med, 32(4), 585-594. doi:10.1017/s0033291702005457

Singh-Manoux, A., Gueguen, A., Ferrie, J., Shipley, M., Martikainen, P., Bonenfant, S., . . Marmot, M. (2008). Gender differences in the association between morbidity and mortality among middle-aged men and women. Am J Public Health, 98(12), 2251-2257. doi:10.2105/AJPH.2006.107912

Singh, K., Osei-Akoto, I., Otchere, F., Sodzi-Tettey, S., Barrington, C., Huang, C., . . Speizer, I. (2015). Ghana's National Health insurance scheme and maternal and child health: a mixed methods study. BMC Health Serv Res, 15, 108. doi:10.1186/s12913-015-0762-y

Skaftun, E. K., Ali, M., \& Norheim, O. F. (2014). Understanding inequalities in child health in Ethiopia: health achievements are improving in the period 2000-2011. PLoS One, 9(8), e106460. doi:10.1371/journal. pone.0106460

Smith, K. V., \& Sulzbach, S. (2008). Community-based health insurance and access to maternal health services: evidence from three West African countries. Soc Sci Med, 66(12), 2460-2473. doi:10.1016/j. socscimed.2008.01.044

Solar, O., \& Irwin, A. (2010). A conceptual framework for action on the social determinants of health. Social Determinants of Health Discussion Paper 2 (Policy and Practice). In.

Some, D. T., Sombie, I., \& Meda, N. (2013). How decision for seeking maternal care is made--a qualitative study in two rural medical districts of Burkina Faso. Reprod Health, 10(1), 8. doi:10.1186/1742-4755-10-8

Starbird, E., Norton, M., \& Marcus, R. (2016). Investing in Family Planning: Key to Achieving the Sustainable Development Goals. Glob Health Sci Pract, 4(2), 191-210. doi:10.9745/GHSP-D-15-00374

StataCorp. (2017). Stata Statistical Software: Release 15. In. College Station, TX: StataCorp LLC.

Stover, J., Hardee, K., Ganatra, B., Garcia Moreno, C., \& Horton, S. (2016). Interventions to Improve Reproductive Health. In R. E. Black, R. Laxminarayan, M. Temmerman, \& N. Walker (Eds.), Reproductive, Maternal, Newborn, and Child Health: Disease Control Priorities, Third Edition (Volume 2). Washington (DC): The International Bank for Reconstruction and Development / The World Bank (c) 2016 International Bank for Reconstruction and Development / The World Bank

The Partnership for Maternal, N. C. H. (2011). Essential Interventions, Commodities and Guidelines for Reproductive, Maternal, Newborn and Child Health. Geneva, Switzerland: PMNCH: World Health Organization.

Thomson, M., Kentikelenis, A., \& Stubbs, T. (2017). Structural adjustment programmes adversely affect vulnerable populations: a systematic-narrative review of their effect on child and maternal health. Public health reviews, 38, 13. doi:10.1186/s40985-017-0059-2

Tsui, A. O., Brown, W., \& Li, Q. (2017). Contraceptive Practice in Sub-Saharan Africa. Popul Dev Rev, 43(Suppl Suppl 1), 166-191. doi:10.1111/padr.12051

United Nations. (2015). Sustainable Development Knowledge Platform. SUSTAINABLE DEVELOPMENT 
GOAL 3 Ensure healthy lives and promote well-being for all at all ages. Retrieved from https:// sustainabledevelopment.un.org/sdg3

United Nations. (2015). Transforming our world: The 2030 agenda for sustainable development. Resolution adopted by the General Assembly. Retrieved from https://sustainabledevelopment.un.org/post2015/ transformingourworld

United Nations Development Programme. (2019). Sustainable Development Goals: Goal 3 targets. Retrieved from https://www.undp.org/content/undp/en/home/sustainable-development-goals/goal-3-goodhealth-and-well-being/targets.html

United Nations Economic Commission for Africa. (2015). Africa Regional Report on the Sustainable Development Goals. Retrieved from https://www.uneca.org/publications/africa-regional-reportsustainable-development-goals

United Nations Population Fund. (2016). Universal access to reproductive health: progress and challenges. New York: UNFPA.

Victora, C. G., Joseph, G., Silva, I. C. M., Maia, F. S., Vaughan, J. P., Barros, F. C., \& Barros, A. J. D. (2018). The Inverse Equity Hypothesis: Analyses of Institutional Deliveries in 286 National Surveys. Am J Public Health, 108(4), 464-471. doi:10.2105/AJPH.2017.304277

Victora, C. G., Vaughan, J. P., Barros, F. C., Silva, A. C., \& Tomasi, E. (2000). Explaining trends in inequities: evidence from Brazilian child health studies. Lancet, 356(9235), 1093-1098. doi:10.1016/S0140-6736(00)02741-0

Wabiri, N., Chersich, M., Shisana, O., Blaauw, D., Rees, H., \& Dwane, N. (2016). Growing inequities in maternal health in South Africa: a comparison of serial national household surveys. BMC Pregnancy Childbirth, 16, 256. doi:10.1186/s12884-016-1048-z

Wagstaff. (1991). On the measurement of Inequalitites in health.

Wallace, L. J., \& Kapiriri, L. (2019). Priority setting for maternal, newborn and child health in Uganda: a qualitative study evaluating actual practice. BMC Health Serv Res, 19(1), 465. doi:10.1186/s12913-019-4170-6

Wang, W., Alva, S., Wang, S., \& Fort, A. (2011). Levels and trends in the use of maternal health services in developing countries. DHS Comparative Reports No. 26. Calverton: US Agency for International Development.

Warenius, L. U., Faxelid, E. A., Chishimba, P. N., Musandu, J. O., Ong'any, A. A., \& Nissen, E. B. M. (2006). NurseMidwives' Attitudes towards Adolescent Sexual and Reproductive Health Needs in Kenya and Zambia. Reproductive Health Matters, 14(27), 119-128. doi:10.1016/s0968-8080(06)27242-2

Whitehead, M. (1992). The concepts and principles of equity and health. Int J Health Serv, 22(3), 429-445. doi:10.2190/986L-LHQ6-2VTE-YRRN

World Health Organisation. (2005a, 08/04/2019). Health and the Millennium Development Goals. Retrieved from https://www.who.int/hdp/publications/mdg_en.pdf?ua=1

World Health Organisation. (2005b). Reducing maternal deaths: the challenge of the new millennium in the African Region. In Brazzaville: Congo: WHO Regional Office for Africa.

World Health Organisation. (2006). Reproductive health indicators: guidelines for their generation, interpretation and analysis for global monitoring. Retrieved from http://www.who.int/reproductivehealth/publications/ monitoring/924156315x/en/ 
World Health Organisation. (2011). PMNCH Fact Sheet: RMNCH Continuum of care. Retrieved from https:// www.who.int/pmnch/about/continuum_of_care/en/

World Health Organisation. (2013a). Health impact assessment, glossary of terms. Retrieved from https://www. who.int/hia/about/glos/en/index1.html

World Health Organisation. (2014). WHO country cooperation strategy 2014-2019: Nigeria. In.

World Health Organisation. (2015a). Health in 2015: from MDGs, millennium development goals to SDGs, sustainable development goals. Retrieved from https://www.who.int/gho/publications/mdgs-sdgs/en/

World Health Organisation. (2015b). Trends in maternal mortality: 1990-2015: estimates from WHO, UNICEF, UNFPA, World Bank Group and the United Nations Population Division. In.

World Health Organisation. (2020a). Burkina Faso. Retrieved from https://www.who.int/countries/bfa/en/ World Health Organisation. (2020b). Ghana. Retrieved from https://www.who.int/countries/gha/en/ World Health Organisation. (2020c). Niger. Retrieved from https://www.who.int/countries/ner/en/ World Health Organisation. (2020d). Nigeria. Retrieved from https://www.who.int/countries/nga/en/ World Health Organisation. (2020e). Senegal. Retrieved from https://www.who.int/countries/sen/en/ World Health Organisation. (2018). The state of health in the WHO African Region: an analysis of the status of health, health services and health systems in the context of the Sustainable Development Goals. In.

World Health Organisation. (2013b). State of health financing in the African Region. In. Brazzaville: World Health Organization. Regional Office for Africa.

Witter, S. (2009). Service-and population-based exemptions: are these the way forward for equity and efficiency in health financing in low-income countries? In Innovations in Health System Finance in Developing and Transitional Economies (2009/10/02 ed., pp. 251-288): Emerald Group Publishing Limited.

Witter, S., Arhinful, D. K., Kusi, A., \& Zakariah-Akoto, S. (2007). The experience of Ghana in implementing a user fee exemption policy to provide free delivery care. Reprod Health Matters, 15(30), 61-71. doi:10.1016/ S0968-8080(07)30325-X

Witter, S., Boukhalfa, C., Cresswell, J. A., Daou, Z., Filippi, V., Ganaba, R., . . team, F. E. (2016). Cost and impact of policies to remove and reduce fees for obstetric care in Benin, Burkina Faso, Mali and Morocco. Int J Equity Health, 15(1), 123. doi:10.1186/s12939-016-0412-y

Witter, S., Dieng, T., Mbengue, D., Moreira, I., \& De Brouwere, V. (2010). The national free delivery and caesarean policy in Senegal: evaluating process and outcomes. Health Policy Plan, 25(5), 384-392. doi:10.1093/ heapol/czq013

Witter, S., \& Garshong, B. (2009). Something old or something new? Social health insurance in Ghana. BMC Int Health Hum Rights, 9(1), 20. doi:10.1186/1472-698X-9-20

World Bank. (2016). Universal Health Coverage (UHC) in Africa: A Framework for Action: Main Report. In W. B. Group (Ed.): The World Bank.

World Bank. (n.d.). Data for Ghana, Nigeria. Retrieved from https://data.worldbank.org/?locations=NG-GH Wulifan, J. K., Mazalale, J., Jahn, A., Hien, H., Ilboudo, P. C., Meda, N., .. De Allegri, M. (2017). Factors Associated with Contraceptive Use among Women of Reproductive Age in Rural Districts of Burkina Faso. J Health Care Poor Underserved, 28(1), 228-247. doi:10.1353/hpu.2017.0019 
REFERENCES

Zere, E., Kirigia, J. M., Duale, S., \& Akazili, J. (2012). Inequities in maternal and child health outcomes and interventions in Ghana. BMC Public Health, 12, 252. doi:10.1186/1471-2458-12-252

Zere, E., Moeti, M., Kirigia, J., Mwase, T., \& Kataika, E. (2007). Equity in health and healthcare in Malawi: analysis of trends. BMC Public Health, 7(1), 78. doi:10.1186/1471-2458-7-78 
REFERENCES 



\section{APPENDIX A}

Additional information for Chapter 2 


\section{Appendix A1}

Description of data: Reproductive health care services in each service category

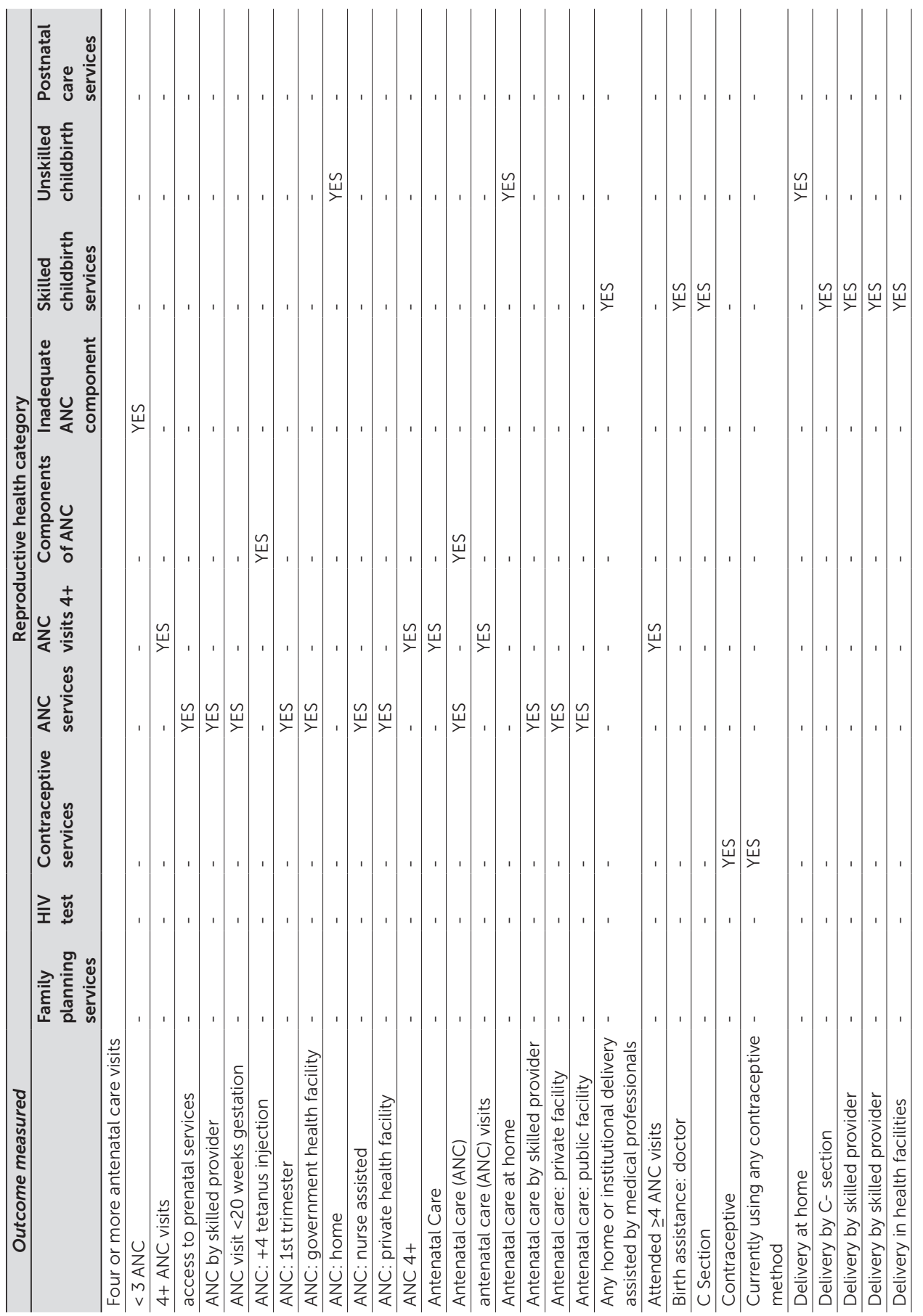




\section{Appendix A1 continued}

Description of data: Reproductive health care services in each service category

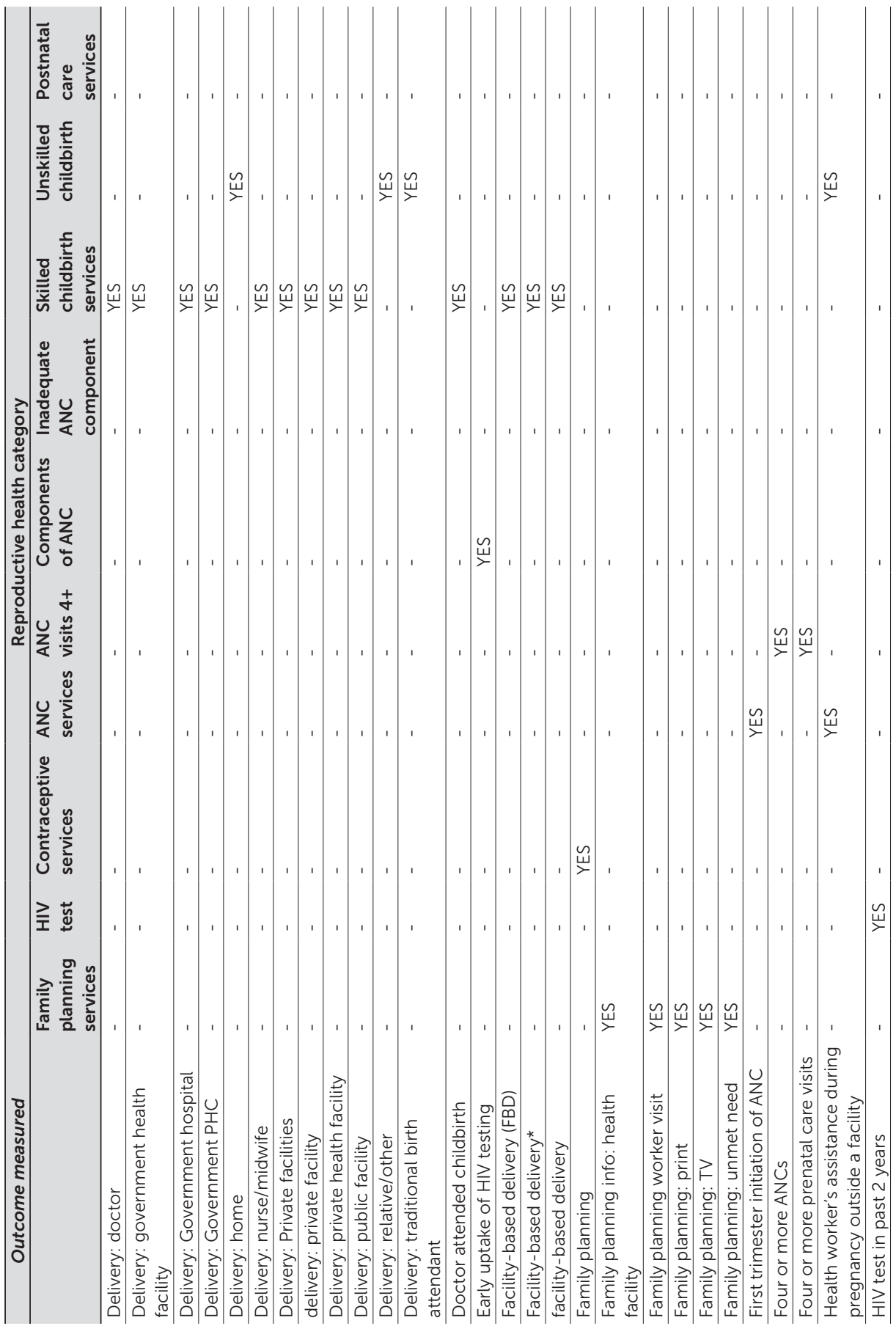




\section{Appendix A1 continued}

Description of data: Reproductive health care services in each service category

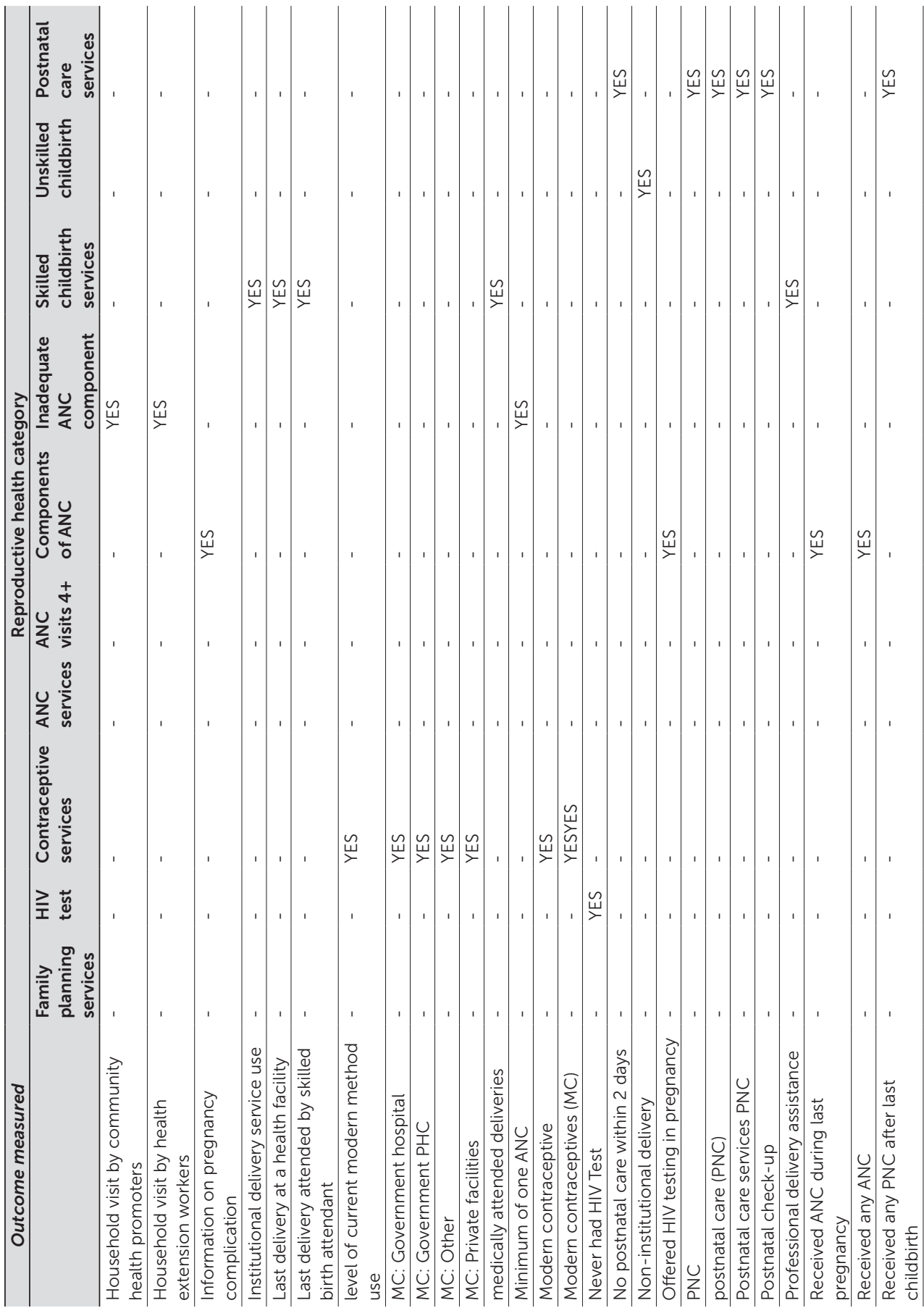




\section{Appendix A1 continued}

Description of data: Reproductive health care services in each service category

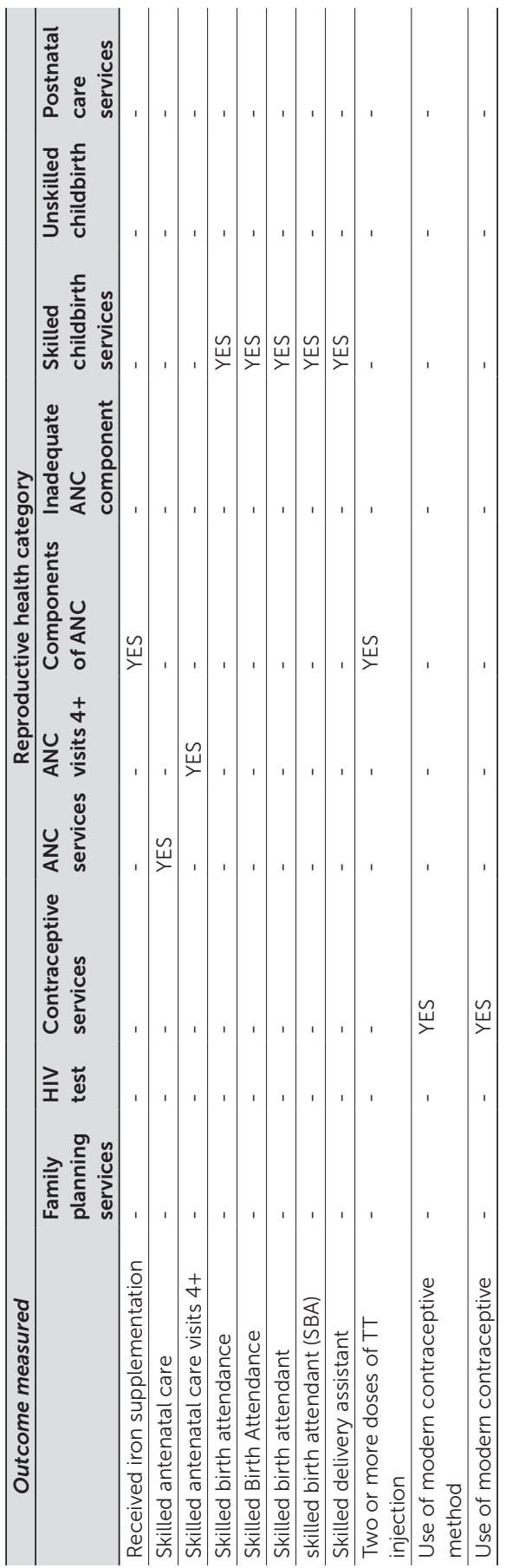




\section{Appendix A2}

Description of data: Publication Reviewed (22 publications reviewed)

1. Agha S, Do M. Does an expansion in private sector contraceptive supply increase inequality in modern contraceptive use? Health Policy Plan. 2008;23(6):465-75.

2. Makoka D. Towards an understanding of regional disparities in social inequities in maternal health in Malawi. African health sciences. 2009;9(4):234-41.

3. Adeyanju O, Tubeuf S, Ensor T. Socio-economic inequalities in access to maternal and child health care in Nigeria: changes over time and decomposition analysis. Health Policy Plan. 2017;32(8):1111-8.

4. Alam N, Hajizadeh M, Dumont A, Fournier P. Inequalities in maternal health care utilization in sub-Saharan African countries: a multiyear and multi-country analysis. PLoS One. 2015;10(4):e0120922.

5. Ambel AA, Andrews C, Bakilana AM, Foster EM, Khan Q, Wang H. Examining changes in maternal and child health inequalities in Ethiopia. Int $\mathrm{J}$ Equity Health. 2017;16(1):152.

6. Bobo FT, Yesuf EA, Woldie M. Inequities in utilization of reproductive and maternal health services in Ethiopia. Int J Equity Health. 2017;16(1):105.

7. Do M, Soelaeman R, Hotchkiss DR. Explaining inequity in the use of institutional delivery services in selected countries. Maternal and child health journal. 2015:19(4):755-63.

8. Gebre E, Worku A, Bukola F. Inequities in maternal health services utilization in Ethiopia 2000-2016: magnitude, trends, and determinants. Reprod Health. 2018;15(1):119.

9. Goli S, Nawal D, Rammohan A, Sekher TV, Singh D. DECOMPOSING THE SOCIOECONOMIC INEQUALITY IN UTILIZATION OF MATERNAL HEALTH CARE SERVICES IN SELECTED COUNTRIES OF SOUTH ASIA AND SUB-SAHARAN AFRICA. J Biosoc Sci. 2018;50(6):749-69.

10. Jalloh MB, Bah AJ, James PB, Sevalie S, Hann K, Shmueli A. Impact of the free health care initiative on wealth-related inequity in the utilization of maternal $\&$ child health services in Sierra Leone. BMC Health Serv Res. 2019;19(1):352.

11. Karim AM, Tamire A, Medhanyie AA, Betemariam W. Changes in equity of maternal, newborn, and child health care practices in 115 districts of rural Ethiopia: implications for the health extension program. Bmc Pregnancy Childb. 2015;15:238.

12. Makate $M$, Makate $C$. The evolution of socioeconomic status-related inequalities in maternal health care utilization: evidence from Zimbabwe, 1994-2011. Glob Health Res Policy. 2017;2:1. 
13. Memirie ST, Verguet S, Norheim OF, Levin C, Johansson KA. Inequalities in utilization of maternal and child health services in Ethiopia: the role of primary health care. BMC Health Serv Res. 2016;16:51.

14. Mezmur M, Navaneetham K, Letamo G, Bariagaber H. Socioeconomic inequalities in the uptake of maternal health care services in Ethiopia. BMC Health Serv Res. 2017;17(1):367.

15. Ngandu NK, Van Malderen C, Goga A, Speybroeck N. Wealth-related inequality in early uptake of HIV testing among pregnant women: an analysis of data from a national cross-sectional survey, South Africa. BMJ open. 2017;7(7):e013362.

16. Obiyan MO, Kumar A. Socioeconomic Inequalities in the Use of Maternal Health Care Services in Nigeria: Trends Between 1990 and 2008. Sage Open. 2015;5(4):2158244015614070.

17. Ogundele OJ, Pavlova M, Groot W. Examining trends in inequality in the use of reproductive health care services in Ghana and Nigeria. Bmc Pregnancy Childb. 2018;18(1):492.

18. Onarheim KH, Taddesse M, Norheim OF, Abdullah M, Miljeteig I. Towards universal health coverage for reproductive health services in Ethiopia: two policy recommendations. Int J Equity Health. 2015;14:86.

19. Van Malderen C, Ogali I, Khasakhala A, Muchiri SN, Sparks C, Van Oyen H, et al. Decomposing Kenyan socio-economic inequalities in skilled birth attendance and measles immunization. Int J Equity Health. 2013;12:3.

20. Wabiri N, Chersich M, Shisana O, Blaauw D, Rees H, Dwane N. Growing inequities in maternal health in South Africa: a comparison of serial national household surveys. Bmc Pregnancy Childb. 2016;16:256.

21. Zere E, Tumusiime $P$, Walker $O$, Kirigia J, Mwikisa C, Mbeeli T. Inequities in utilization of maternal health interventions in Namibia: implications for progress towards MDG 5 targets. Int J Equity Health. 2010;9:16.

22. Mwase T, Brenner S, Mazalale J, Lohmann J, Hamadou S, Somda SMA, et al. Inequities and their determinants in coverage of maternal health services in Burkina Faso. International Journal for Equity in Health. 2018;17(1):58. 


\section{Appendix A3}

Description of data: Fisher's z units Forest plots for the association between socioeconomic inequality and family planning services.

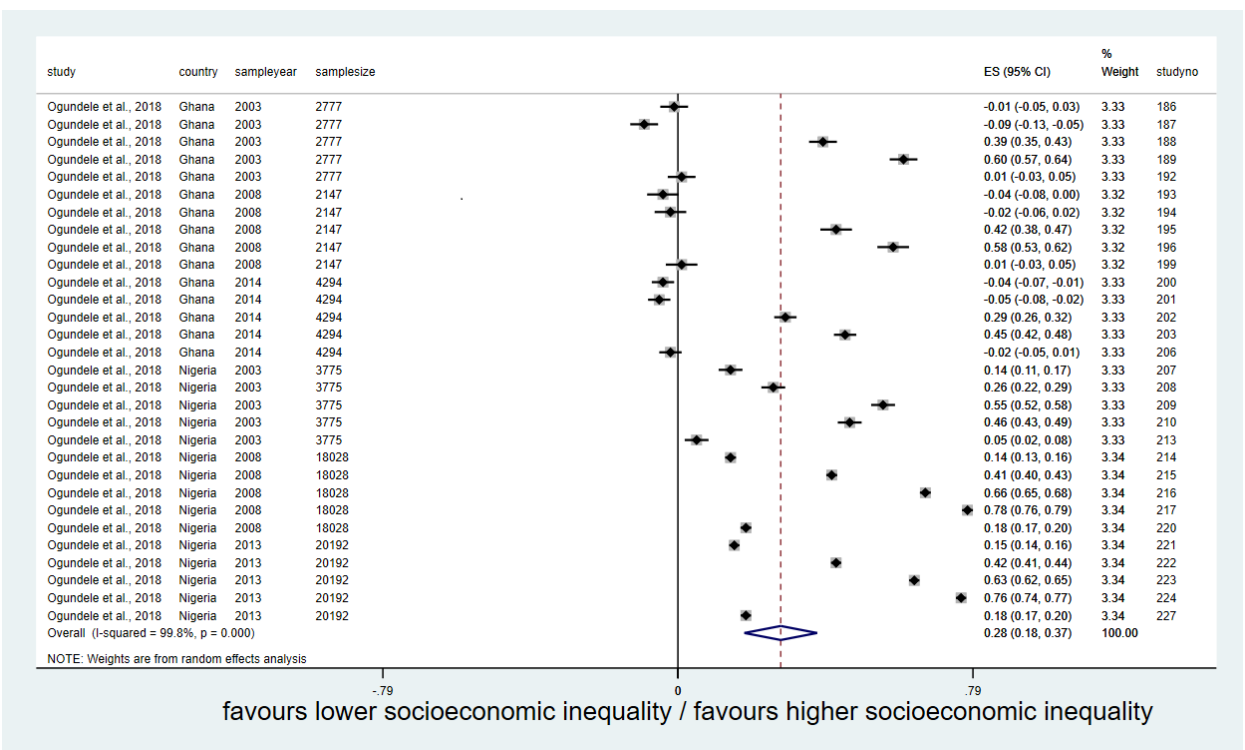

Description of data: Fisher's z units Forest plots for the association between socioeconomic inequality and contraceptive services.

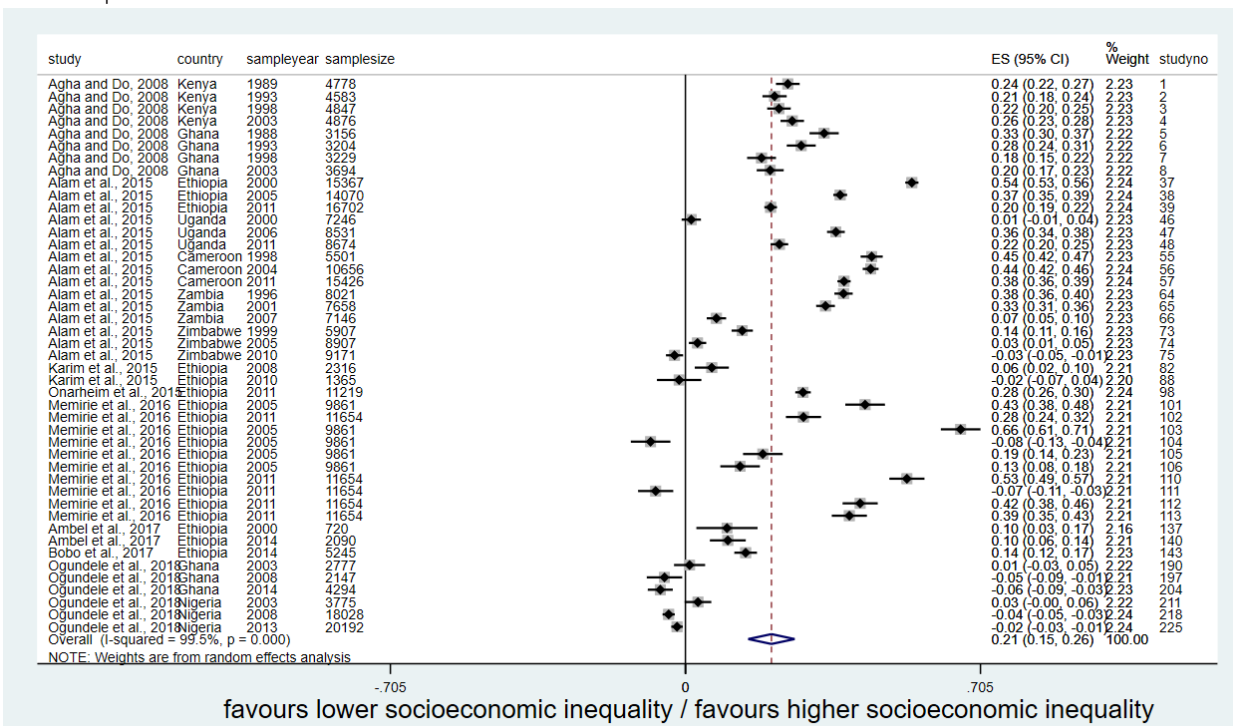


Description of data: Fisher's z units Forest plots for the association between socioeconomic inequality and antenatal care services.

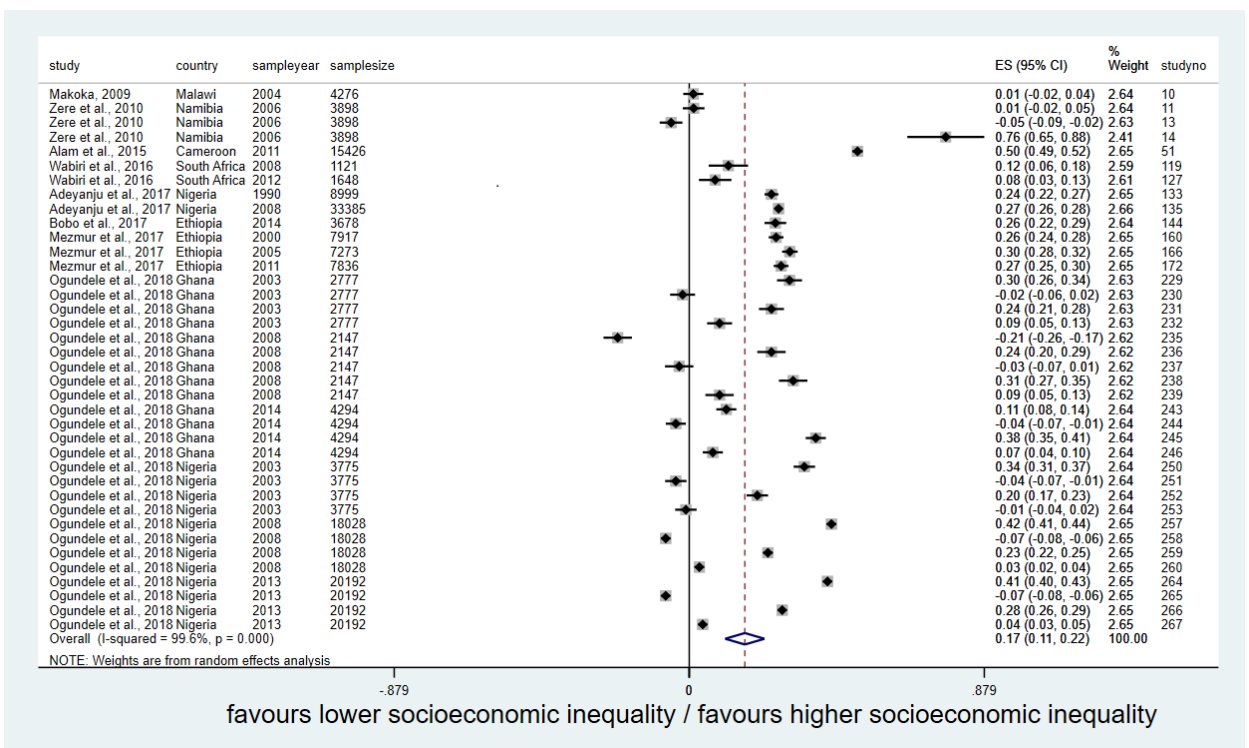

Description of data: Fisher's z units Forest plots for the association between socioeconomic inequality and components of antenatal care.

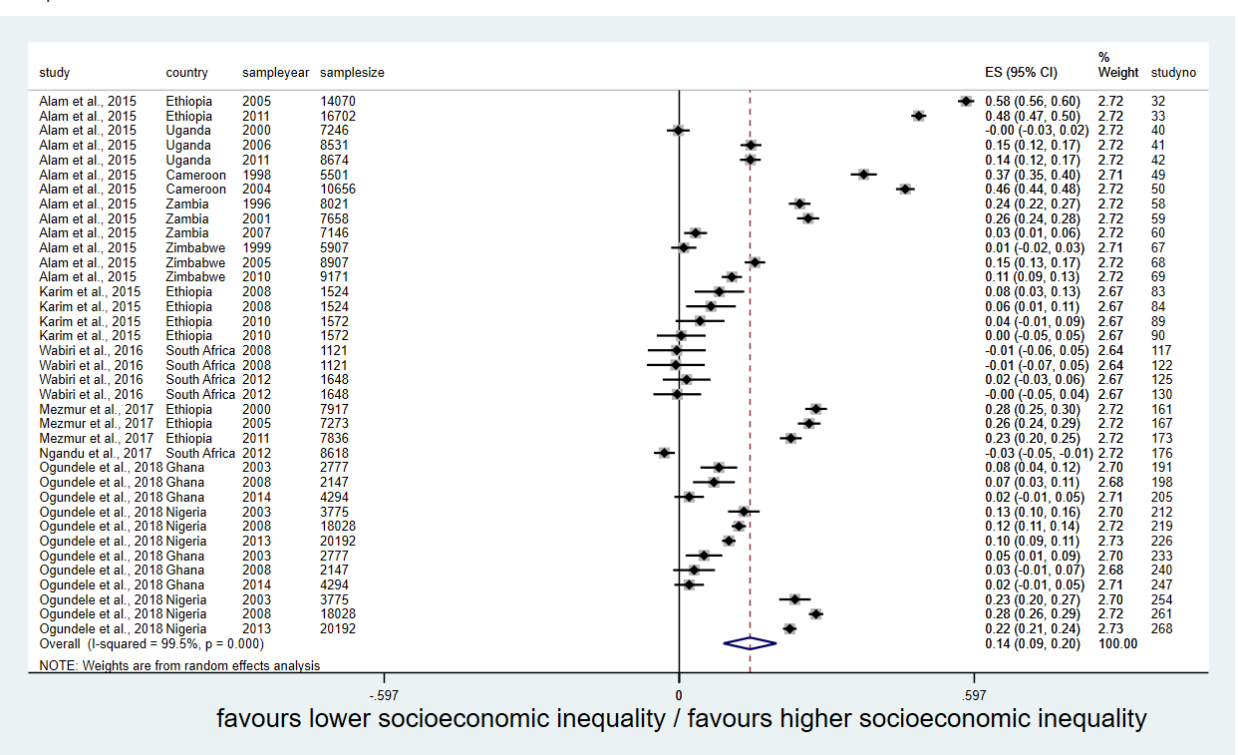


Description of data: Fisher's z units Forest plots for the association between socioeconomic inequality and skilled childbirth services.

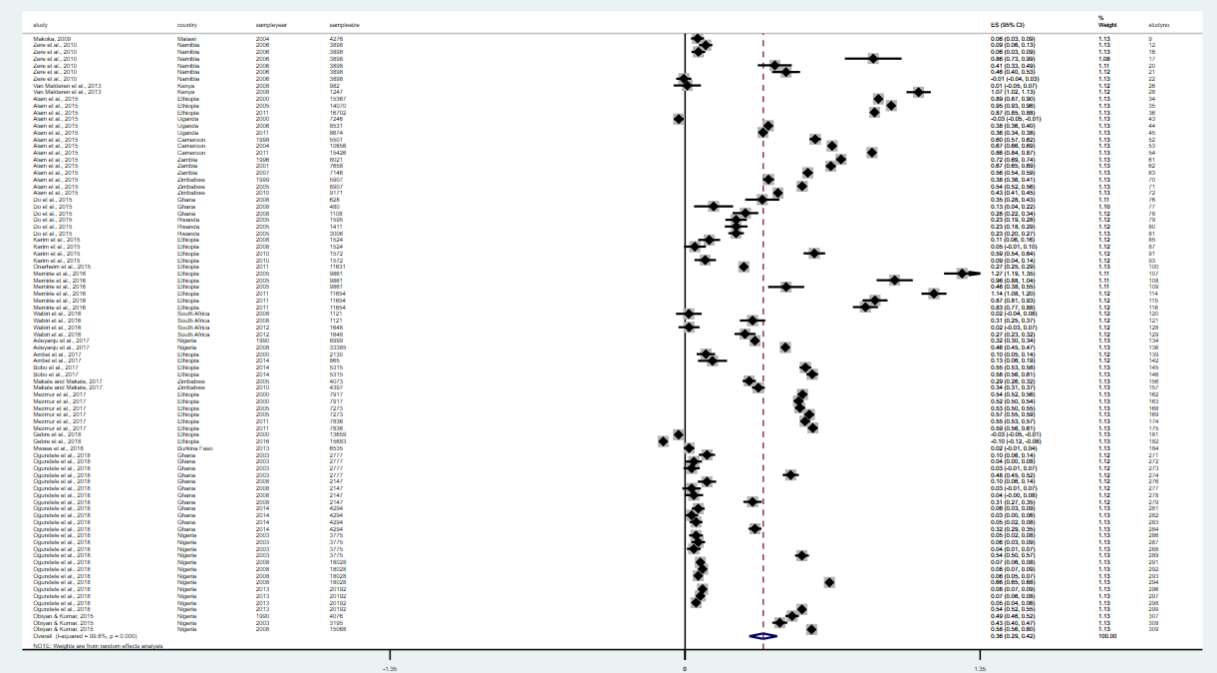

favours lower socioeconomic inequality / favours higher socioeconomic inequality 
APPENDIX A - FOR CHAPTER 2 



\section{APPENDIX B}

Additional information for Chapter 3 


\section{Appendix B1 Cluster composition variables.}

Description of data: Cluster composition variables family planning: Nigeria and Ghana

\begin{tabular}{|c|c|c|c|c|c|c|c|c|c|c|c|}
\hline & & Nigeria & & & & & Ghana & & & & \\
\hline $\begin{array}{l}\text { Cluster Variable } \\
\text { composition }\end{array}$ & Value range & $\mathrm{N}(\%)$ & Mean & Median & SD & N Obs. & $\mathrm{N}(\%)$ & Mean & Median & SD & N Obs. \\
\hline \multirow[t]{2}{*}{$\begin{array}{l}\text { Ever tried to delay or } \\
\text { avoid pregnancy }\end{array}$} & $\begin{array}{l}1=\mathrm{No} / \text { Used } \\
\text { outside calendar }\end{array}$ & $\begin{array}{l}4485 \\
(58.1)\end{array}$ & 1.42 & 1 & 0.49 & 7725 & $\begin{array}{l}2284 \\
(55.1)\end{array}$ & 1.45 & 1 & 0.50 & 4142 \\
\hline & $\begin{array}{l}2=\text { Yes in } \\
\text { calendar }\end{array}$ & $\begin{array}{l}3241 \\
(41.9)\end{array}$ & & & & & $\begin{array}{l}1858 \\
(44.9)\end{array}$ & & & & \\
\hline $\begin{array}{l}\text { Using modern } \\
\text { contraceptive }\end{array}$ & $\begin{array}{l}1=\text { Using } \\
\text { modern }\end{array}$ & $\begin{array}{l}1523 \\
(19.7)\end{array}$ & & & & & $\begin{array}{l}1047 \\
(25.7)\end{array}$ & & & & \\
\hline $\begin{array}{l}\text { Use traditional } \\
\text { contraceptive }\end{array}$ & $\mathrm{O}=\mathrm{No}$ & $\begin{array}{l}6933 \\
(89.7)\end{array}$ & 0.21 & 0 & 0.61 & 7725 & & & & & \\
\hline $\begin{array}{l}\text { Visited by family } \\
\text { planning worker last } \\
12 \text { months }\end{array}$ & $1=$ Yes & $\begin{array}{l}1799 \\
(23.3)\end{array}$ & & & & & & & & & \\
\hline \multirow[t]{2}{*}{$\begin{array}{l}\text { FP services needed } \\
\text { covered by NHIS }\end{array}$} & $0=$ Not covered & & & & & & $\begin{array}{l}2773 \\
(67.0)\end{array}$ & 0.99 & 0 & 1.41 & 4142 \\
\hline & $1=$ Covered & & & & & & $\begin{array}{l}1369 \\
(33.0)\end{array}$ & & & & \\
\hline \multirow[t]{2}{*}{$\begin{array}{l}\text { Lab services needed } \\
\text { covered by NHIS }\end{array}$} & $0=$ Not covered & & & & & & $\begin{array}{l}4000 \\
(96.6)\end{array}$ & 0.03 & 0 & 0.18 & 4141 \\
\hline & $1=$ Covered & & & & & & $\begin{array}{l}140 \\
(3.4)\end{array}$ & & & & \\
\hline $\begin{array}{l}\text { Intends to use } \\
\text { contraceptive later }\end{array}$ & $1=$ Yes & $\begin{array}{l}2394 \\
(31.0)\end{array}$ & & & & & $52(1.3)$ & & & & \\
\hline
\end{tabular}


Description of data: Cluster variables maternal health care services: Nigeria and Ghana

\begin{tabular}{|c|c|c|c|c|c|c|c|c|c|c|c|}
\hline \multirow{2}{*}{$\begin{array}{c}\text { Cluster variable } \\
\text { composition }\end{array}$} & \multicolumn{3}{|c|}{ Nigeria } & \multirow[b]{2}{*}{ Median } & \multirow[b]{2}{*}{ SD } & \multirow[b]{2}{*}{ N Obs. } & \multicolumn{4}{|l|}{ Ghana } & \multirow[b]{2}{*}{ N Obs. } \\
\hline & Value range & $\mathrm{N}(\%)$ & Mean & & & & $\mathrm{N}(\%)$ & Mean & Median & SD & \\
\hline \multirow{2}{*}{$\begin{array}{l}\text { Told about } \\
\text { pregnancy } \\
\text { complications }\end{array}$} & $\begin{array}{l}0=\mathrm{No} / \text { Don't } \\
\text { know }\end{array}$ & $1682(21.8)$ & 0.78 & 1 & 0.41 & 7662 & $627(15.1)$ & 0.84 & 1 & 0.36 & 4014 \\
\hline & $1=$ Yes & $5980(77.4)$ & & & & & 3387 (81.8) & & & & \\
\hline \multirow{2}{*}{$\begin{array}{l}\text { Place of delivery } \\
\text { home }\end{array}$} & $0=\mathrm{No} /$ other & $6148(79.6)$ & 0.20 & 0 & 0.40 & 7725 & $3131(75.6)$ & 0.24 & 0 & 0.43 & 4142 \\
\hline & $1=$ Yes & $1578(20.4)$ & & & & & $1011(24.4)$ & & & & \\
\hline \multirow{2}{*}{$\begin{array}{l}\text { Place of delivery: } \\
\text { govt. public } \\
\text { facility }\end{array}$} & $\mathrm{O}=\mathrm{No} /$ other & 3766 (48.8) & 10.76 & 1 & 10.50 & 7725 & $1387(33.5)$ & 13.97 & 1 & 9.91 & 4142 \\
\hline & $1=$ Yes & 3959 (51.2) & & & & & 2755 (66.5) & & & & \\
\hline \multirow{2}{*}{$\begin{array}{l}\text { Place of delivery: } \\
\text { private } \& \text { other }\end{array}$} & $0=\mathrm{No} /$ other & $5537(71.7)$ & 8.78 & 0 & 13.97 & 7725 & 3767 (90.9) & 2.81 & 0 & 8.90 & 4142 \\
\hline & $1=$ Yes & 2189 (28.3) & & & & & $375(9.1)$ & & & & \\
\hline \multirow{2}{*}{$\begin{array}{l}\text { Birth assistance: } \\
\text { TBA }\end{array}$} & $0=\mathrm{No}$ & $7142(92.5)$ & 0.07 & 0 & 0.26 & 7713 & $3542(85.5)$ & 0.14 & 0 & 0.35 & 4142 \\
\hline & $1=$ Yes & $570(7.4)$ & & & & & $600(14.5)$ & & & & \\
\hline \multirow{2}{*}{$\begin{array}{l}\text { ANC: Govt. } \\
\text { hospital }\end{array}$} & $0=\mathrm{No}$ & $4373(56.6)$ & 0.43 & 0 & 0.50 & 7725 & 2045 (49.4) & 0.49 & 0 & 0.50 & 4036 \\
\hline & $1=$ Yes & $3352(43.4)$ & & & & & $1991(48.1)$ & & & & \\
\hline \multirow{2}{*}{$\begin{array}{l}\text { ANC: government } \\
\text { health center }\end{array}$} & $\mathrm{O}=\mathrm{No}$ & $5608(72.6)$ & 0.27 & 0 & 0.45 & 7725 & $2575(62.2)$ & 0.36 & 0 & 0.48 & 4036 \\
\hline & $1=$ Yes & 2117 (27.4) & & & & & $1461(35.3)$ & & & & \\
\hline \multirow{2}{*}{$\begin{array}{l}\text { ANC: government } \\
\text { health post/ } \\
\text { dispensary }\end{array}$} & $\mathrm{O}=\mathrm{No}$ & 7569 (98.0) & 0.02 & 0 & 0.14 & 7725 & 3806 (91.9) & 0.06 & 0 & 0.23 & 4036 \\
\hline & $1=Y e s$ & $156(2.0)$ & & & & & $229(5.5)$ & & & & \\
\hline \multirow{2}{*}{$\begin{array}{l}\text { ANC: Private } \\
\text { hospital/clinic }\end{array}$} & $\mathrm{O}=\mathrm{No}$ & $5498(71.2)$ & 0.29 & 0 & 0.45 & 7725 & 3607 (87.1) & 0.11 & 0 & 0.31 & 4036 \\
\hline & $1=$ Yes & $2228(28.8)$ & & & & & $428(10.3)$ & & & & \\
\hline \multirow{2}{*}{$\begin{array}{l}\text { ANC: respondent's } \\
\text { home }\end{array}$} & $\mathrm{O}=\mathrm{No}$ & 7694 (99.6) & 0.00 & 0 & 0.06 & 7725 & & & & & \\
\hline & $1=$ Yes & $31(0.4)$ & & & & & & & & & \\
\hline \multirow{2}{*}{$\begin{array}{l}\text { ANC: other private } \\
\text { medical sector }\end{array}$} & $\mathrm{O}=\mathrm{No}$ & 7690 (99.5) & 0.00 & 0 & 0.07 & 7725 & & & & & \\
\hline & $1=$ Yes & $35(0.5)$ & & & & & & & & & \\
\hline \multirow[t]{2}{*}{ Assistance: doctor } & $\mathrm{O}=\mathrm{No}$ & $5716(74)$ & 0.26 & 0 & 0.44 & 7713 & & & & & \\
\hline & $1=$ Yes & $1996(25.8)$ & & & & & & & & & \\
\hline \multirow[t]{2}{*}{$\begin{array}{l}\text { Assistance: no } \\
\text { one }\end{array}$} & $\begin{array}{l}0=\text { No: some } \\
\text { assistance }\end{array}$ & 7596 (98.3) & 0.02 & 0 & 0.12 & 7713 & & & & & \\
\hline & $\begin{array}{l}\text { 1= Yes: no } \\
\text { assistance }\end{array}$ & $117(1.5)$ & & & & & & & & & \\
\hline \multirow{2}{*}{$\begin{array}{l}\text { Check-up after } \\
\text { delivery }\end{array}$} & $\mathrm{O}=\mathrm{No}$ & & & & & & $564(13.6)$ & 0.86 & 1 & 0.34 & 4140 \\
\hline & $1=$ Yes & & & & & & $3576(86.3)$ & & & & \\
\hline \multirow[t]{2}{*}{ ANC: CHPS } & $\mathrm{O}=\mathrm{No}$ & & & & & & 3806 (91.9) & 0.06 & 0 & 0.23 & 4036 \\
\hline & $1=$ Yes & & & & & & $229(5.5)$ & & & & \\
\hline
\end{tabular}



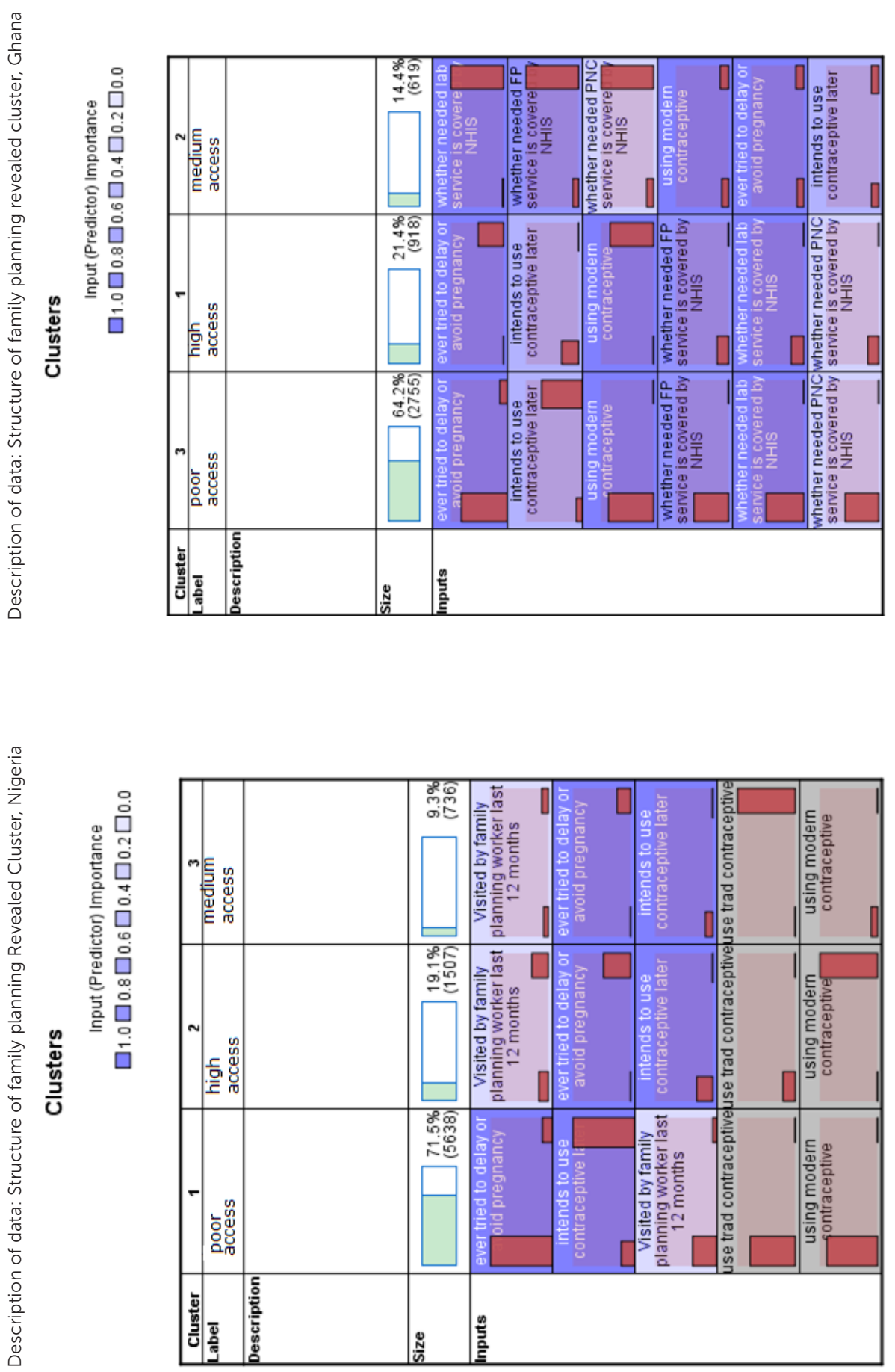

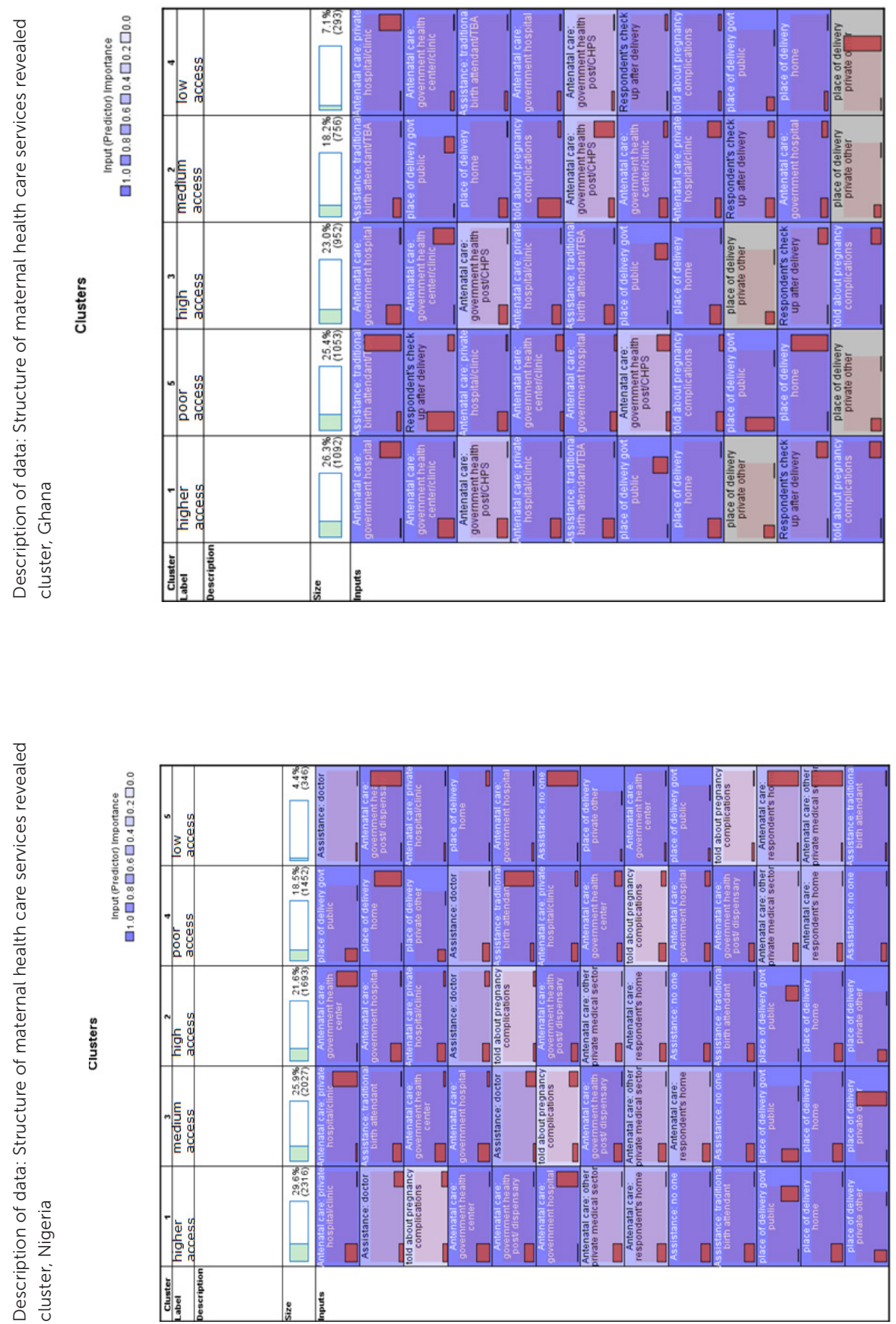


\section{APPENDIX B2}

Description of data: Background characteristics socioeconomic characteristics of Nigeria and Ghana samples

\begin{tabular}{|c|c|c|c|c|c|c|c|c|c|}
\hline & & NIGERIA & & & & GHANA & & & \\
\hline Variable & Value range & $\mathrm{N}(\%)$ & Mean & SD & N Obs. & $\mathrm{N}(\%)$ & Mean & SD & N Obs. \\
\hline Maternal age & 15- 49 years & & 29.92 & 6.91 & 7725 & & 30.64 & 7.03 & 4142 \\
\hline Number of children alive & $0-13$ persons & & 3.09 & 1.97 & 7725 & & 3.04 & 1.87 & 4142 \\
\hline \multirow[t]{3}{*}{ Marital status } & $0=$ Never married & $251(3.2)$ & 3.83 & 0.73 & 7725 & $393(9.5)$ & 3.55 & 1.18 & 4142 \\
\hline & $\begin{array}{l}\text { 1= Widowed/separated/ } \\
\text { divorced }\end{array}$ & $281(3.6)$ & & & & $304(7.3)$ & & & \\
\hline & $2=$ Married & 7194 (93.1) & & & & 3446 (83.2) & & & \\
\hline \multirow[t]{3}{*}{ Maternal Education } & $0=$ No education & $1345(17.4)$ & 1.43 & 0.77 & 7725 & $1079(26)$ & 1.28 & 0.85 & 4142 \\
\hline & $1=$ Primary & $1712(22.2)$ & & & & $812(19.6)$ & & & \\
\hline & 2= Secondary / Higher & $4668(60.4)$ & & & & $2251(54.3)$ & & & \\
\hline \multirow[t]{5}{*}{ Maternal Occupation } & $1=$ Not working & $1684(21.8)$ & 2.65 & 1.18 & 7696 & $730(17.6)$ & 3.64 & 1.78 & 4142 \\
\hline & $2=$ services and manual & $1389(18)$ & & & & $596(14.4)$ & & & \\
\hline & $3=$ sales & $3193(41.3)$ & & & & $1566(37.8)$ & & & \\
\hline & $4=$ agriculture & $761(9.8)$ & & & & $1039(25.1)$ & & & \\
\hline & $5=$ white collar & $669(8.7)$ & & & & $210(5.1)$ & & & \\
\hline \multirow[t]{5}{*}{ Household Wealth } & $1=$ Poorest & $2839(36.7)$ & 3.81 & 1.17 & 7725 & $869(21)$ & 2.96 & 1.42 & 4142 \\
\hline & $2=$ Poorer & 2157 (27.9) & & & & $840(20.3)$ & & & \\
\hline & $3=$ Middle & $1528(19.8)$ & & & & $827(20)$ & & & \\
\hline & $4=$ Richer & $853(11)$ & & & & $814(19.7)$ & & & \\
\hline & $5=$ Richest & $349(4.5)$ & & & & $791(19.1)$ & & & \\
\hline \multirow[t]{2}{*}{ Residence } & $1=$ Rural & $4462(57.8)$ & 1.42 & 0.49 & 7725 & $2228(53.8)$ & 2.46 & 0.50 & 4142 \\
\hline & $2=$ Urban & 3264 (42.2) & & & & $1914(46.2)$ & & & \\
\hline \multirow[t]{2}{*}{ Has health insurance } & $\mathrm{O}=\mathrm{No}$ & $7420(96)$ & 0.04 & 0.19 & 7703 & $1376(33.2)$ & 0.67 & 0.47 & 4142 \\
\hline & $1=$ Yes & $283(3.7)$ & & & & $2766(66.8)$ & & & \\
\hline \multirow[t]{4}{*}{ Religion } & 1= Catholic & $962(12.5)$ & 3.23 & 0.97 & 7684 & $411(9.9)$ & 5.81 & 1.70 & 4142 \\
\hline & $2=$ Traditionalist & $33(0.4)$ & & & & $278(6.7)$ & & & \\
\hline & $3=$ Islam & $3001(38.8)$ & & & & $696(16.8)$ & & & \\
\hline & $4=$ Other Christian & $3688(47.7)$ & & & & $2757(66.6)$ & & & \\
\hline \multirow{2}{*}{$\begin{array}{l}\text { Need permission for } \\
\text { medical help }\end{array}$} & 1= Big problem & $358(4.6)$ & 1.95 & 0.21 & 7693 & $578(14)$ & 1.86 & 0.35 & 4142 \\
\hline & $2=$ Not $a$ big problem & 7335 (94.9) & & & & $3564(86)$ & & & \\
\hline \multirow{2}{*}{$\begin{array}{l}\text { Need money for medical } \\
\text { help }\end{array}$} & 1= Big problem & $2641(34.2)$ & 1.66 & 0.48 & 7693 & $238(5.8)$ & 1.94 & 0.23 & 4142 \\
\hline & $2=$ Not $a$ big problem & $5051(65.4)$ & & & & 3904 (94.2) & & & \\
\hline \multirow{2}{*}{$\begin{array}{l}\text { Distance to health } \\
\text { facility }\end{array}$} & 1= Big problem & 1349 (17.5) & 1.82 & 0.38 & 7699 & $1803(43.5)$ & 1.56 & 0.50 & 4142 \\
\hline & $2=$ Not $a$ big problem & $6350(82.2)$ & & & & 2339 (56.5) & & & \\
\hline \multirow{2}{*}{$\begin{array}{l}\text { Do not want to visit } \\
\text { health facility alone }\end{array}$} & 1= Big problem & $484(6.3)$ & 1.94 & 0.24 & 7692 & 1099 (26.5) & 1.73 & 0.44 & 4142 \\
\hline & $2=$ Not $a$ big problem & 7208 (93.3) & & & & $3043(73.5)$ & & & \\
\hline
\end{tabular}


Background characteristics (continued)

\begin{tabular}{|c|c|c|c|c|c|c|c|c|c|}
\hline & & NIGERIA & & & & GHANA & & & \\
\hline Variable & Value range & $\mathrm{N}(\%)$ & Mean & SD & N Obs. & $\mathrm{N}(\%)$ & Mean & SD & N Obs. \\
\hline \multirow[t]{2}{*}{ Attitude of the health workers } & $1=$ Big problem & 867 (11.2) & 1.89 & 0.32 & 7695 & & & & \\
\hline & $\begin{array}{l}2=\text { Not } a \text { big } \\
\text { problem }\end{array}$ & $6828(88.4)$ & & & & & & & \\
\hline \multirow{2}{*}{$\begin{array}{l}\text { Heard family planning on } \\
\text { radio last few months }\end{array}$} & $0=\mathrm{No}$ & 3789 (49) & 0.51 & 0.50 & 7716 & $1682(40.6)$ & 0.59 & 0.49 & 4142 \\
\hline & $1=Y e s$ & $3927(50.8)$ & & & & $2460(59.4)$ & & & \\
\hline \multirow{2}{*}{$\begin{array}{l}\text { Heard family planning on TV } \\
\text { last few months }\end{array}$} & $0=\mathrm{No}$ & $5041(65.3)$ & 0.35 & 0.48 & 7708 & $2128(51.4)$ & 0.49 & 0.50 & 4142 \\
\hline & $1=$ Yes & $2666(34.5)$ & & & & $2014(48.6)$ & & & \\
\hline \multirow{2}{*}{$\begin{array}{l}\text { Heard family planning in print } \\
\text { last few months }\end{array}$} & $0=\mathrm{No}$ & $6850(88.7)$ & 0.11 & 0.31 & 7695 & 3959 (95.6) & 0.04 & 0.21 & 4142 \\
\hline & $1=$ Yes & 845 (10.9) & & & & $183(4.4)$ & & & \\
\hline \multirow[t]{13}{*}{ Ethnicity } & $2=$ Other minorities & 3086 (39.9) & 3.82 & 1.66 & 7725 & & & & \\
\hline & $3=$ Fulani & $205(2.7)$ & & & & & & & \\
\hline & $4=\lg b o$ & $1446(18.7)$ & & & & & & & \\
\hline & $5=$ Hausa & $1011(13.1)$ & & & & & & & \\
\hline & $6=$ Yoruba & $1977(25.6)$ & & & & & & & \\
\hline & $2=\mathrm{Ga} /$ Dangme & & & & & $266(6.4)$ & 7.17 & 2.98 & 4142 \\
\hline & $3=$ Ewe & & & & & $548(13.2)$ & & & \\
\hline & $4=$ Guan & & & & & $80(1.9)$ & & & \\
\hline & $5=$ Mole - Dagbani & & & & & 717 (17.3) & & & \\
\hline & $6=$ Grusi & & & & & $123(3)$ & & & \\
\hline & $7=$ Gurma & & & & & $319(7.7)$ & & & \\
\hline & $8=$ Mande & & & & & $128(3.1)$ & & & \\
\hline & $10=$ Akan & & & & & $1962(47.4)$ & & & \\
\hline \multirow[t]{16}{*}{ Region } & $1=$ North Central & $1290(16.7)$ & 3.86 & 1.85 & 7725 & & & & \\
\hline & $2=$ North East & $884(11.4)$ & & & & & & & \\
\hline & $3=$ North West & $1097(14.2)$ & & & & & & & \\
\hline & $4=$ South East & 1040 (13.5) & & & & & & & \\
\hline & $5=$ South South & $1143(14.8)$ & & & & & & & \\
\hline & $6=$ South West & $2271(29.4)$ & & & & & & & \\
\hline & $1=$ Western & & & & & $427(10.3)$ & 4.87 & 2.51 & 4142 \\
\hline & $2=$ Central & & & & & 455 (11) & & & \\
\hline & $4=$ Volta & & & & & $315(7.6)$ & & & \\
\hline & $5=$ Eastern & & & & & $389(9.4)$ & & & \\
\hline & $6=$ Ashanti & & & & & $738(17.8)$ & & & \\
\hline & $7=$ Brong Ahafo & & & & & $374(9)$ & & & \\
\hline & $8=$ Northern & & & & & 480 (11.6) & & & \\
\hline & $9=$ Upper East & & & & & $178(4.3)$ & & & \\
\hline & $10=$ Upper West & & & & & $111(2.7)$ & & & \\
\hline & $11=$ Greater Accra & & & & & $674(16.3)$ & & & \\
\hline
\end{tabular}




\section{Appendix B3 Full results of the regression analyses.}

Description of data: Odds ratio family planning Ghana

\begin{tabular}{|c|c|c|c|c|c|c|}
\hline \multirow[b]{2}{*}{ Background characteristics } & \multicolumn{3}{|c|}{$\begin{array}{l}\text { Medium access } \\
\text { (high access reference) }\end{array}$} & \multicolumn{3}{|c|}{$\begin{array}{l}\text { Poor access } \\
\text { (high access reference) }\end{array}$} \\
\hline & B & S.E. & Exp B $(95 \% \mathrm{CI})$ & B & S.E. & $\operatorname{Exp~B~}(95 \% \mathrm{Cl})$ \\
\hline Intercept & $-0.793^{\star}$ & 0.461 & & 0.301 & 0.356 & \\
\hline Maternal age & $0.046^{\star \star \star}$ & 0.012 & $1.047(1.023-1.072)$ & $0.036^{\star * *}$ & 0.009 & $1.037(1.019-1.055)$ \\
\hline Number of children alive & $-0.161^{\star \star \star}$ & 0.046 & $0.851(0.777-0.931)$ & $-0.163^{\star \star \star}$ & 0.034 & $0.849(0.795-0.908)$ \\
\hline \multicolumn{7}{|l|}{ Marital status } \\
\hline \multicolumn{7}{|l|}{ Married (reference) } \\
\hline Never married & 0.065 & 0.212 & $1.068(0.705-1.617)$ & $0.307 * *$ & 0.153 & $1.359(1.008-1.833)$ \\
\hline Widowed/separated/divorced & 0.004 & 0.217 & $1.004(0.656-1.535)$ & -0.067 & 0.151 & $0.935(0.695-1.258)$ \\
\hline \multicolumn{7}{|l|}{ Maternal Education } \\
\hline \multicolumn{7}{|l|}{ Secondary/ Higher (reference) } \\
\hline No education & 0.300 & 0.184 & $1.35(0.941-1.938)$ & $0.423^{\star \star *}$ & 0.135 & $1.527(1.173-1.988)$ \\
\hline Primary & -0.202 & 0.157 & $0.817(0.601-1.112)$ & -0.040 & 0.110 & $0.961(0.774-1.192)$ \\
\hline \multicolumn{7}{|l|}{ Maternal Occupation } \\
\hline \multicolumn{7}{|l|}{ White collar (reference) } \\
\hline Not working & $0.549 * *$ & 0.267 & $1.732(1.027-2.921)$ & $0.786^{\star \star *}$ & 0.212 & $2.194(1.447-3.325)$ \\
\hline Services and manual & 0.204 & 0.270 & $1.227(0.723-2.081)$ & $0.546^{\star * *}$ & 0.213 & 1.727 (1.137- 2.622) \\
\hline Sales & 0.213 & 0.241 & $1.238(0.771-1.987)$ & $0.522^{\star \star \star}$ & 0.194 & $1.686(1.152-2.466)$ \\
\hline Agriculture & 0.321 & 0.287 & $1.378(0.785-2.421)$ & $0.588^{\star \star \star}$ & 0.223 & $1.801(1.164-2.786)$ \\
\hline \multicolumn{7}{|l|}{ Household Wealth } \\
\hline \multicolumn{7}{|l|}{ Richest (reference) } \\
\hline Poorest & -0.054 & 0.307 & $0.947(0.519-1.731)$ & 0.339 & 0.229 & $1.403(0.895-2.200)$ \\
\hline Poorer & -0.247 & 0.260 & $0.781(0.469-1.302)$ & 0.138 & 0.193 & $1.148(0.786-1.677)$ \\
\hline Middle & -0.255 & 0.216 & $0.775(0.508-1.183)$ & -0.003 & 0.164 & $0.997(0.723-1.375)$ \\
\hline Richer & -0.075 & 0.180 & $0.928(0.652-1.32)$ & -0.032 & 0.141 & $0.968(0.734-1.277)$ \\
\hline \multicolumn{7}{|l|}{ Residence } \\
\hline \multicolumn{7}{|l|}{ Urban (reference) } \\
\hline Rural & $-0.291^{*}$ & 0.151 & $0.748(0.556-1.006)$ & $-0.249 * *$ & 0.113 & $0.779(0.624-0.974)$ \\
\hline \multicolumn{7}{|l|}{ Has health insurance } \\
\hline \multicolumn{7}{|l|}{ Yes (reference) } \\
\hline No & $-1.138^{\star \star \star}$ & 0.134 & $0.320(0.246-0.417)$ & $-0.188^{* *}$ & 0.087 & $0.829(0.699-0.983)$ \\
\hline \multicolumn{7}{|l|}{ Religion } \\
\hline \multicolumn{7}{|l|}{ Other Christian (reference) } \\
\hline Catholic & -0.194 & 0.188 & $0.824(0.57-1.19)$ & -0.116 & 0.141 & $0.891(0.676-1.173)$ \\
\hline Traditionalist/ none & 0.120 & 0.208 & $1.128(0.75-1.695)$ & 0.128 & 0.154 & $1.137(0.840-1.538)$ \\
\hline Islam & $-0.641^{* \star}$ & 0.292 & $0.527(0.297-0.932)$ & 0.104 & 0.179 & 1.109 (0.781- 1.575) \\
\hline \multicolumn{7}{|l|}{ Need permission for medical help } \\
\hline \multicolumn{7}{|l|}{ Not a big problem (reference) } \\
\hline Big problem & 0.036 & 0.246 & $1.036(0.64-1.678)$ & 0.015 & 0.186 & $1.015(0.705-1.462)$ \\
\hline \multicolumn{7}{|l|}{ Need money for medical help } \\
\hline \multicolumn{7}{|l|}{ Not a big problem (reference) } \\
\hline Big problem & 0.260 * & 0.133 & $1.297(0.999-1.682)$ & 0.050 & 0.098 & $1.051(0.868-1.273)$ \\
\hline \multicolumn{7}{|l|}{ Distance to health facility } \\
\hline Not a big problem (reference) & & & & & & \\
\hline Big problem & 0.213 & 0.158 & $1.237(0.908-1.685)$ & 0.152 & 0.118 & $1.164(0.923-1.466)$ \\
\hline Do not want to visit health facility & & & & & & \\
\hline Not a big problem (reference) & & & & & & \\
\hline Big problem & 0.017 & 0.187 & $1.017(0.705-1.465)$ & 0.020 & 0.143 & $1.020(0.771-1.349)$ \\
\hline
\end{tabular}


Odds ratio family planning Ghana (continued)

\begin{tabular}{|c|c|c|c|c|c|c|}
\hline \multirow[b]{2}{*}{ Background characteristics } & \multicolumn{3}{|c|}{$\begin{array}{l}\text { Medium access } \\
\text { (high access reference) }\end{array}$} & \multicolumn{3}{|c|}{$\begin{array}{l}\text { Poor access } \\
\text { (high access reference) }\end{array}$} \\
\hline & B & S.E. & $\operatorname{Exp} B(95 \% \mathrm{Cl})$ & B & S.E. & $\operatorname{Exp} B(95 \% \mathrm{Cl})$ \\
\hline \multicolumn{7}{|c|}{ Heard family planning on radio last few months } \\
\hline \multicolumn{7}{|l|}{ Yes (reference) } \\
\hline No & 0.094 & 0.132 & $1.099(0.848-1.424)$ & 0.023 & 0.097 & $1.023(0.846-1.236)$ \\
\hline \multicolumn{7}{|c|}{ Heard family planning on TV last few months } \\
\hline No & -0.190 & 0.145 & $0.827(0.623-1.098)$ & 0.011 & 0.107 & $1.012(0.819-1.249)$ \\
\hline \multirow{2}{*}{\multicolumn{7}{|c|}{$\begin{array}{l}\text { Heard family planning in print last few months } \\
\text { Yes (reference) }\end{array}$}} \\
\hline & & & & & & \\
\hline No & $-0.594^{* *}$ & 0.267 & $0.552(0.327-0.933)$ & -0.342 & 0.224 & $0.710(0.458-1.103)$ \\
\hline \multicolumn{7}{|l|}{ Region } \\
\hline \multicolumn{7}{|l|}{ Greater Accra (reference) } \\
\hline Western & $0.555^{\star \star}$ & 0.236 & $1.742(1.097-2.765)$ & -0.142 & 0.180 & $0.868(0.610-1.234)$ \\
\hline Central & -0.282 & 0.249 & $0.754(0.463-1.229)$ & $-0.370 * \star$ & 0.171 & $0.691(0.494-0.966)$ \\
\hline Volta & $1.160 * \star *$ & 0.267 & $3.191(1.890-5.389)$ & $-0.696^{* * *}$ & 0.218 & $0.499(0.326-0.764)$ \\
\hline Eastern & $-0.746^{\star \star \star}$ & 0.282 & $0.474(0.273-0.825)$ & -0.141 & 0.175 & $0.868(0.617-1.223)$ \\
\hline Ashanti & $0.648^{\star * *}$ & 0.211 & $1.912(1.264-2.893)$ & 0.098 & 0.159 & $1.103(0.808-1.505)$ \\
\hline Brong Ahafo & $0.602^{\star \star}$ & 0.248 & $1.826(1.123-2.969)$ & $-0.418^{\star *}$ & 0.192 & $0.658(0.451-0.960)$ \\
\hline Northern & 0.485 & 0.339 & $1.623(0.835-3.155)$ & $0.590 * \star$ & 0.260 & $1.805(1.083-3.006)$ \\
\hline Upper East & $-1.923^{\star \star \star}$ & 0.599 & $0.146(0.045-0.473)$ & -0.344 & 0.262 & $0.709(0.424-1.185)$ \\
\hline Upper West & $-1.338^{\star *}$ & 0.549 & $0.262(0.090-0.769)$ & -0.318 & 0.308 & $0.728(0.398-1.332)$ \\
\hline \multicolumn{7}{|l|}{ Ethnicity (Ghana) } \\
\hline \multicolumn{7}{|l|}{ Akan (reference) } \\
\hline Ga/Dangme & 0.258 & 0.261 & $1.294(0.775-2.159)$ & 0.133 & 0.188 & $1.142(0.790-1.650)$ \\
\hline Ewe & 0.043 & 0.213 & $1.044(0.687-1.584)$ & 0.057 & 0.156 & $1.059(0.780-1.437)$ \\
\hline Guan & 0.489 & 0.374 & $1.631(0.783-3.396)$ & -0.124 & 0.323 & $0.883(0.469-1.664)$ \\
\hline Mole-Dagbani & 0.169 & 0.230 & $1.184(0.754-1.860)$ & 0.060 & 0.174 & $1.061(0.754-1.493)$ \\
\hline Grusi & -0.060 & 0.334 & $0.942(0.490-1.811)$ & -0.383 & 0.245 & $0.682(0.422-1.103)$ \\
\hline Gurma & -0.053 & 0.304 & $0.948(0.523-1.720)$ & -0.125 & 0.230 & $0.883(0.563-1.385)$ \\
\hline Mande & -0.578 & 0.380 & $0.561(0.266-1.181)$ & -0.300 & 0.259 & $0.741(0.446-1.230)$ \\
\hline \multicolumn{7}{|l|}{ Attitude of the health workers } \\
\hline \multicolumn{7}{|l|}{ Big problem } \\
\hline \multicolumn{7}{|l|}{ Not a big problem (reference) } \\
\hline \multicolumn{7}{|l|}{ REGRESSION STATISTICS } \\
\hline$N$ observations & 4142 & & 4142 & & & \\
\hline-2 LL Ratio (Final) & $6947.847^{\star * *}$ & & $6947.847 * \star \star$ & & & \\
\hline Chi-Square (Pearson) & 559.727 & & 559.727 & & & \\
\hline Pseudo R Squared (McFadden) & 0.074 & & 0.074 & & & \\
\hline
\end{tabular}


Description of data: Odds ratio family planning: Nigeria

\begin{tabular}{|c|c|c|c|c|c|c|}
\hline \multirow[b]{2}{*}{ Background characteristics } & \multicolumn{3}{|c|}{$\begin{array}{l}\text { Medium access } \\
\text { (high access reference) }\end{array}$} & \multicolumn{3}{|c|}{$\begin{array}{l}\text { Poor access } \\
\text { (high access reference) }\end{array}$} \\
\hline & B & S.E. & $\operatorname{Exp~B~}(95 \% \mathrm{Cl})$ & B & S.E. & $\operatorname{Exp~B~}(95 \% \mathrm{CI})$ \\
\hline Intercept & $-1.137^{\star \star \star}$ & 0.396 & & -0.094 & 0.285 & \\
\hline Maternal age & $0.018^{*}$ & 0.010 & $1.018(0.998-1.038)$ & $0.015^{\star *}$ & 0.007 & $1.015(1.001-1.029)$ \\
\hline Number of children alive & $-0.061^{*}$ & 0.035 & $0.940(0.878-1.007)$ & $-0.256^{\star \star \star}$ & 0.025 & $0.774(0.738-0.813)$ \\
\hline \multicolumn{7}{|l|}{ Marital status } \\
\hline \multicolumn{7}{|l|}{ Married (reference) } \\
\hline Never married & $-1.084^{\star \star \star}$ & 0.308 & $0.338(0.185-0.619)$ & $-0.598^{* * *}$ & 0.169 & $0.550(0.395-0.767)$ \\
\hline Widowed/separated/divorced & 0.037 & 0.274 & $1.038(0.606-1.777)$ & $0.426^{\star \star}$ & 0.191 & $1.531(1.052-2.228)$ \\
\hline \multicolumn{7}{|l|}{ Maternal Education } \\
\hline \multicolumn{7}{|l|}{ Secondary/ Higher (reference) } \\
\hline No education & 0.229 & 0.246 & $1.257(0.776-2.037)$ & $0.934 * \star \star$ & 0.147 & $2.544(1.907-3.395)$ \\
\hline Primary & -0.192 & 0.128 & $0.825(0.642-1.061)$ & 0.105 & 0.086 & $1.111(0.939-1.314)$ \\
\hline \multicolumn{7}{|l|}{ Maternal Occupation } \\
\hline \multicolumn{7}{|l|}{ White collar (reference) } \\
\hline Not working & 0.006 & 0.181 & $1.006(0.706-1.435)$ & 0.126 & 0.128 & $1.135(0.882-1.459)$ \\
\hline Services and manual & 0.125 & 0.174 & $1.134(0.806-1.594)$ & $0.249 * \star$ & 0.126 & $1.283(1.002-1.642)$ \\
\hline Sales & -0.073 & 0.154 & $0.930(0.687-1.258)$ & -0.013 & 0.112 & $0.987(0.793-1.228)$ \\
\hline Agriculture & -0.216 & 0.241 & $0.805(0.503-1.291)$ & 0.178 & 0.164 & $1.194(0.867-1.646)$ \\
\hline \multicolumn{7}{|l|}{ Household Wealth } \\
\hline \multicolumn{7}{|l|}{ Richest (reference) } \\
\hline Poorest & -0.272 & 0.633 & $0.762(0.220-2.633)$ & $1.229 * * *$ & 0.320 & $3.417(1.825-6.396)$ \\
\hline Poorer & 0.026 & 0.256 & $1.026(0.621-1.694)$ & $0.825^{\star \star \star}$ & 0.160 & $2.282(1.669-3.120)$ \\
\hline Middle & 0.216 & 0.164 & $1.241(0.899-1.714)$ & $0.683^{\star \star *}$ & 0.114 & $1.979(1.583-2.475)$ \\
\hline Richer & $0.209 *$ & 0.118 & $1.232(0.977-1.553)$ & $0.533^{\star * *}$ & 0.083 & $1.704(1.448-2.006)$ \\
\hline \multicolumn{7}{|l|}{ Residence } \\
\hline \multicolumn{7}{|l|}{ Urban (reference) } \\
\hline Rural & 0.088 & 0.120 & $1.092(0.864-1.381)$ & -0.076 & 0.084 & $0.927(0.785-1.093)$ \\
\hline \multicolumn{7}{|l|}{ Has health insurance } \\
\hline \multicolumn{7}{|l|}{ Yes (reference) } \\
\hline No & -0.095 & 0.210 & $0.909(0.602-1.373)$ & $0.318^{* *}$ & 0.156 & $1.374(1.011-1.867)$ \\
\hline \multicolumn{7}{|l|}{ Religion } \\
\hline \multicolumn{7}{|l|}{ Other Christian (reference) } \\
\hline Catholic & 0.076 & 0.145 & $1.078(0.811-1.433)$ & 0.047 & 0.108 & $1.048(0.849-1.295)$ \\
\hline Traditionalist/ none & 0.280 & 0.842 & $1.324(0.254-6.898)$ & 0.715 & 0.604 & $2.043(0.625-6.678)$ \\
\hline Islam & 0.172 & 0.133 & $1.188(0.915-1.542)$ & $0.388^{\star * *}$ & 0.088 & $1.474(1.240-1.753)$ \\
\hline \multicolumn{7}{|l|}{ Need permission for medical help } \\
\hline \multicolumn{7}{|l|}{ Not a big problem (reference) } \\
\hline Big problem & 0.220 & 0.245 & $1.247(0.771-2.016)$ & 0.122 & 0.181 & $1.130(0.792-1.611)$ \\
\hline
\end{tabular}

${ }^{* * *} p \leq 0.01 ;{ }^{* \star} p \leq 0.05 ;{ }^{*} p \leq 0.10$ (two-tailed test of significance) 
Odds ratio family planning: Nigeria (continued)

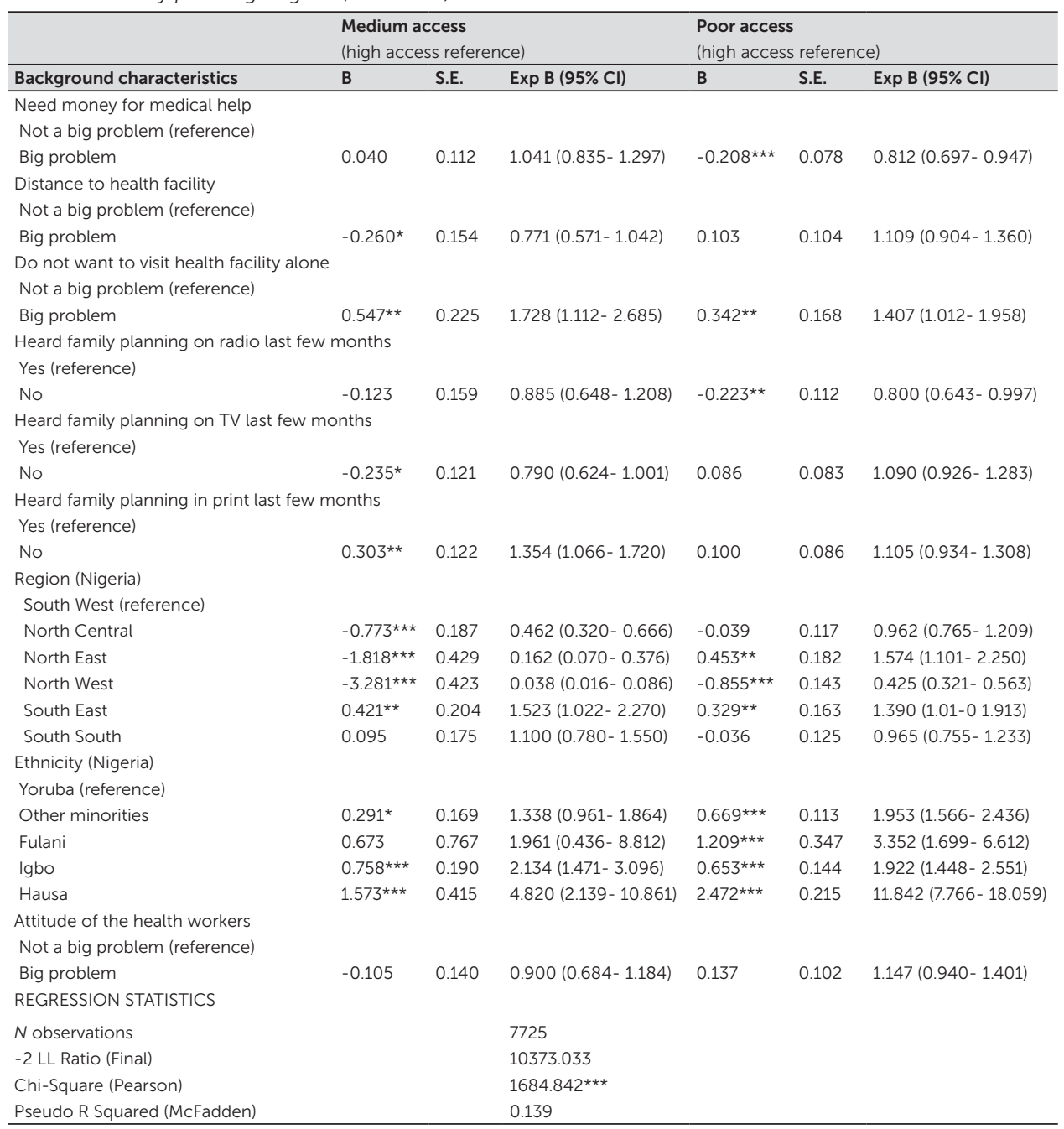

${ }^{* * *} p \leq 0.01 ;{ }^{* *} p \leq 0.05 ;{ }^{*} p \leq 0.10$ (two-tailed test of significance) 
Description of data: Odds ratio maternal health: Ghana

\begin{tabular}{|c|c|c|c|c|c|c|}
\hline \multirow[t]{2}{*}{ Background characteristics } & \multicolumn{3}{|c|}{ High access } & \multicolumn{3}{|c|}{ Middle access } \\
\hline & B & S.E. & $\operatorname{Exp} B(95 \% \mathrm{Cl})$ & B & S.E. & Exp B $(95 \% \mathrm{Cl})$ \\
\hline \multicolumn{7}{|l|}{ Higher access (reference) } \\
\hline Intercept & $-1.020 * *$ & 0.430 & & $-1.132^{\star \star \star}$ & 0.417 & \\
\hline Maternal age & $-0.024^{\star \star}$ & 0.010 & 0.977(0.957-0.997) & -0.017 & 0.011 & $0.984(0.963-1.005)$ \\
\hline Number of children alive & $0.099 * \star$ & 0.043 & $1.105(1.016-1.201)$ & $0.084^{*}$ & 0.044 & $1.088(0.998-1.185)$ \\
\hline \multicolumn{7}{|l|}{ Marital status } \\
\hline Never married & -0.019 & 0.173 & $0.981(0.699-1.377)$ & $-0.338^{*}$ & 0.186 & $0.713(0.495-1.026)$ \\
\hline Widowed/separated/ divorced & -0.023 & 0.188 & $0.978(0.676-1.413)$ & -0.141 & 0.197 & $0.869(0.59-1.278)$ \\
\hline \multicolumn{7}{|l|}{ Married (reference) } \\
\hline \multicolumn{7}{|l|}{ Maternal Education } \\
\hline Primary & -0.04 & 0.160 & $0.961(0.702-1.316)$ & -0.038 & 0.169 & $0.962(0.691-1.341)$ \\
\hline Secondary/ higher & -0.026 & 0.139 & $0.975(0.742-1.28)$ & 0.184 & 0.140 & $1.202(0.914-1.58)$ \\
\hline \multicolumn{7}{|l|}{ Higher (reference) } \\
\hline \multicolumn{7}{|l|}{ Ethnicity } \\
\hline Ga/Dangme & $0.465^{\star \star}$ & 0.204 & $1.592(1.068-2.374)$ & 0.252 & 0.234 & $1.286(0.813-2.035)$ \\
\hline Ewe & $-0.435^{\star \star}$ & 0.200 & $0.647(0.438-0.957)$ & $0.385^{\star \star}$ & 0.187 & $1.469(1.019-2.119)$ \\
\hline Guan & 0.434 & 0.421 & $1.544(0.676-3.525)$ & $1.324^{\star \star \star}$ & 0.412 & $3.76(1.678-8.424)$ \\
\hline Mole-Dagbani & -0.283 & 0.216 & $0.754(0.494-1.15)$ & 0.330 & 0.207 & $1.391(0.927-2.088)$ \\
\hline Grusi & -0.162 & 0.310 & $0.85(0.463-1.561)$ & 0.097 & 0.322 & $1.102(0.587-2.07)$ \\
\hline Gurma & -0.229 & 0.304 & $0.795(0.438-1.443)$ & 0.223 & 0.327 & $1.249(0.658-2.373)$ \\
\hline Mande & -0.504 & 0.328 & $0.604(0.318-1.149)$ & 0.229 & 0.335 & $1.257(0.651-2.425)$ \\
\hline \multicolumn{7}{|l|}{ Akan (reference) } \\
\hline \multicolumn{7}{|l|}{ Maternal Occupation } \\
\hline Not working & 0.071 & 0.261 & $1.074(0.644-1.79)$ & -0.260 & 0.248 & $0.771(0.474-1.254)$ \\
\hline Services and manual & 0.155 & 0.262 & 1.168(0.699-1.952) & -0.246 & 0.250 & $0.782(0.479-1.276)$ \\
\hline Sales & -0.194 & 0.244 & $0.824(0.511-1.329)$ & $-0.551^{\star \star}$ & 0.229 & $0.576(0.368-0.903)$ \\
\hline Agriculture & $0.577^{* \star}$ & 0.283 & $1.781(1.022-3.104)$ & -0.308 & 0.278 & $0.735(0.426-1.266)$ \\
\hline \multicolumn{7}{|l|}{ White collar (reference) } \\
\hline \multicolumn{7}{|l|}{ Household Wealth } \\
\hline Poorest & $1.317^{\star \star \star}$ & 0.276 & $3.732(2.171-6.415)$ & $1.342^{\star \star \star}$ & 0.292 & $3.826(2.16-6.775)$ \\
\hline Poorer & $0.922^{\star \star \star}$ & 0.229 & $2.514(1.605-3.938)$ & $0.855^{\star \star \star}$ & 0.236 & $2.351(1.479-3.737)$ \\
\hline Middle & $0.676^{\star * *}$ & 0.187 & $1.967(1.363-2.838)$ & $0.521^{\star \star *}$ & 0.193 & $1.684(1.153-2.459)$ \\
\hline Richer & 0.234 & 0.158 & $1.263(0.926-1.723)$ & $0.303^{\star}$ & 0.160 & $1.354(0.991-1.851)$ \\
\hline \multicolumn{7}{|l|}{ Richest (reference) } \\
\hline \multicolumn{7}{|l|}{ Residence } \\
\hline Rural & 0.139 & 0.130 & $1.149(0.889-1.483)$ & $0.265^{\star}$ & 0.135 & $1.303(0.999-1.699)$ \\
\hline \multicolumn{7}{|l|}{ Urban (reference) } \\
\hline \multicolumn{7}{|l|}{ Region } \\
\hline Western & $-0.996^{\star \star *}$ & 0.226 & $0.369(0.237-0.576)$ & $0.367^{\star}$ & 0.214 & $1.444(0.948-2.198)$ \\
\hline Central & -0.006 & 0.204 & $0.994(0.666-1.483)$ & $0.467^{\star \star \star}$ & 0.220 & $1.596(1.036-2.459)$ \\
\hline Volta & 0.125 & 0.250 & $1.133(0.695-1.849)$ & $-0.734^{\star \star \star}$ & 0.286 & $0.48(0.274-0.841)$ \\
\hline Eastern & $-0.812^{\star \star \star}$ & 0.221 & $0.444(0.288-0.684)$ & 0.205 & 0.216 & $1.228(0.804-1.874)$ \\
\hline Ashanti & -0.106 & 0.179 & $0.900(0.633-1.279)$ & $0.888^{\star * \star}$ & 0.188 & $2.429(1.681-3.511)$ \\
\hline Brong Ahafo & $-0.434^{\star}$ & 0.227 & $0.648(0.415-1.01)$ & $0.534^{\star \star}$ & 0.235 & $1.705(1.075-2.705)$ \\
\hline Northern & $0.711^{\star \star}$ & 0.286 & $2.036(1.163-3.564)$ & -0.283 & 0.346 & $0.753(0.382-1.484)$ \\
\hline Upper East & $1.298 * \star \star$ & 0.329 & $3.661(1.92-6.98)$ & 0.149 & 0.388 & $1.161(0.542-2.486)$ \\
\hline Upper West & 0.581 & 0.470 & $1.789(0.713-4.489)$ & $2.010 * * \star$ & 0.433 & $7.466(3.195-17.443)$ \\
\hline Greater Accra (reference) & & & & & & \\
\hline Has health insurance & & & & & & \\
\hline No & 0.136 & 0.110 & $1.146(0.923-1.422)$ & $0.231^{\star \star}$ & 0.113 & $1.26(1.009-1.573)$ \\
\hline Yes (reference) & & & & & & \\
\hline Religion & & & & & & \\
\hline Catholic & $-0.342^{\star \star}$ & 0.174 & $0.710(0.505-0.998)$ & $-0.36^{\star \star}$ & 0.178 & $0.698(0.492-0.99)$ \\
\hline Islam & 0.03 & 0.184 & $1.031(0.719-1.478)$ & -0.270 & 0.192 & $0.763(0.524-1.113)$ \\
\hline Traditionalist or religion & 0.107 & 0.279 & $1.112(0.644-1.921)$ & 0.258 & 0.296 & $1.294(0.724-2.314)$ \\
\hline Other Christian (reference) & & & & & & \\
\hline
\end{tabular}


Odds ratio maternal health: Ghana (continued)

\begin{tabular}{|c|c|c|c|c|c|}
\hline \multicolumn{3}{|c|}{ Poor access } & \multicolumn{3}{|l|}{ Low access } \\
\hline B & S.E. & $\operatorname{Exp~B~}(95 \% \mathrm{Cl})$ & B & S.E. & Exp B $(95 \% \mathrm{Cl})$ \\
\hline 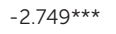 & 0.552 & & $-2.274 * \star \star$ & 0.544 & \\
\hline$-0.040 * * \star$ & 0.011 & $0.961(0.94-0.983)$ & 0.020 & 0.013 & $1.020(0.995-1.047)$ \\
\hline $0.214^{\star \star \star *}$ & 0.045 & $1.239(1.134-1.353)$ & 0.000 & 0.054 & $1.000(0.899-1.113)$ \\
\hline-0.128 & 0.192 & $0.880(0.604-1.281)$ & -0.031 & 0.222 & $0.97(0.628-1.498)$ \\
\hline-0.128 & 0.203 & $0.880(0.591-1.31)$ & -0.220 & 0.265 & $0.803(0.477-1.35)$ \\
\hline $0.433^{\star \star \star}$ & 0.166 & $1.542(1.115-2.132)$ & $-0.385^{\star}$ & 0.234 & $0.680(0.43-1.076)$ \\
\hline $0.322^{\star *}$ & 0.146 & $1.38(1.036-1.838)$ & $-0.521^{\star \star}$ & 0.204 & $0.594(0.398-0.886)$ \\
\hline 0.277 & 0.271 & $1.319(0.776-2.243)$ & 0.376 & 0.250 & $1.456(0.892-2.376)$ \\
\hline 0.143 & 0.21 & $1.154(0.764-1.743)$ & $0.49 * *$ & 0.213 & $1.633(1.076-2.477)$ \\
\hline 0.494 & 0.454 & $1.639(0.674-3.988)$ & $1.961^{\star \star \star}$ & 0.434 & $7.107(3.037-16.632)$ \\
\hline 0.054 & 0.228 & $1.056(0.676-1.649)$ & $0.473^{\star}$ & 0.265 & $1.605(0.955-2.698)$ \\
\hline 0.107 & 0.347 & $1.113(0.564-2.199)$ & 0.054 & 0.445 & $1.056(0.441-2.527)$ \\
\hline 0.363 & 0.305 & $1.438(0.792-2.613)$ & -0.074 & 0.528 & $0.929(0.33-2.615)$ \\
\hline 0.224 & 0.353 & $1.252(0.627-2.497)$ & -0.175 & 0.461 & $0.840(0.34-2.071)$ \\
\hline 0.073 & 0.391 & $1.076(0.500-2.313)$ & -0.118 & 0.259 & $0.889(0.535-1.476)$ \\
\hline 0.132 & 0.394 & $1.141(0.527-2.468)$ & $-0.828^{* \star *}$ & 0.281 & $0.437(0.252-0.757)$ \\
\hline-0.250 & 0.379 & $0.779(0.371-1.636)$ & $-0.465^{\star \star}$ & 0.231 & $0.628(0.4-0.987)$ \\
\hline 0.204 & 0.399 & $1.226(0.561-2.682)$ & $-0.617^{*}$ & 0.340 & $0.539(0.277-1.05)$ \\
\hline $3.027^{\star \star \star}$ & 0.365 & $20.631(10.086-42.199)$ & 0.636 & 0.400 & $1.889(0.863-4.135)$ \\
\hline $2.325^{\star \star \star}$ & 0.33 & $10.228(5.352-19.544)$ & $0.518^{*}$ & 0.291 & $1.679(0.949-2.97)$ \\
\hline $1.853^{\star \star \star}$ & 0.305 & $6.376(3.508-11.587)$ & 0.074 & 0.216 & $1.077(0.705-1.645)$ \\
\hline 0.284 & 0.317 & $1.328(0.713-2.474)$ & $-0.466^{\star * \star}$ & 0.173 & $0.627(0.447-0.881)$ \\
\hline $0.76^{\star \star \star}$ & 0.148 & $2.139(1.600-2.860)$ & $-0.437^{\star \star}$ & 0.181 & $0.646(0.453-0.921)$ \\
\hline 0.293 & 0.285 & $1.340(0.767-2.342)$ & 0.012 & 0.246 & $1.013(0.625-1.64)$ \\
\hline $0.743^{\star \star \star}$ & 0.281 & $2.101(1.211-3.648)$ & 0.158 & 0.248 & $1.171(0.721-1.904)$ \\
\hline-0.181 & 0.313 & $0.834(0.452-1.539)$ & $-0.89 * \star \star$ & 0.348 & $0.411(0.208-0.811)$ \\
\hline 0.226 & 0.274 & $1.253(0.733-2.142)$ & $-0.562^{\star *}$ & 0.275 & $0.57(0.333-0.977)$ \\
\hline 0.089 & 0.279 & $1.093(0.633-1.888)$ & $0.507^{\star \star \star *}$ & 0.196 & $1.66(1.131-2.435)$ \\
\hline-0.138 & 0.306 & $0.871(0.478-1.587)$ & $0.525^{\star \star}$ & 0.261 & $1.69(1.014-2.818)$ \\
\hline $1.086^{\star * \star}$ & 0.351 & $2.962(1.489-5.891)$ & $-1.492^{* \star}$ & 0.587 & $0.225(0.071-0.711)$ \\
\hline-0.408 & 0.429 & $0.665(0.287-1.541)$ & -0.272 & 0.570 & $0.762(0.249-2.329)$ \\
\hline $1.051^{\star \star}$ & 0.497 & $2.861(1.079-7.586)$ & -1.056 & 1.200 & $0.348(0.033-3.654)$ \\
\hline $0.635^{\star \star \star}$ & 0.117 & $1.888(1.500-2.376)$ & 0.189 & 0.140 & $1.209(0.919-1.59)$ \\
\hline$-0.388^{\star *}$ & 0.187 & $0.679(0.470-0.980)$ & $-0.701^{\star \star \star}$ & 0.256 & $0.496(0.3-0.82)$ \\
\hline-0.248 & 0.199 & $0.781(0.528-1.154)$ & -0.192 & 0.256 & $0.825(0.5-1.363)$ \\
\hline $0.808^{* \star *}$ & 0.259 & $2.244(1.351-3.726)$ & $0.905^{\star \star \star}$ & 0.343 & $2.472(1.263-4.837)$ \\
\hline
\end{tabular}


Odds ratio maternal health: Ghana (continued)

\begin{tabular}{|c|c|c|c|c|c|c|}
\hline \multirow[t]{2}{*}{ Background characteristics } & \multicolumn{3}{|c|}{ High access } & \multicolumn{3}{|c|}{ Middle access } \\
\hline & B & S.E. & $\operatorname{Exp~B~}(95 \% \mathrm{Cl})$ & B & S.E. & Exp B $(95 \% \mathrm{Cl})$ \\
\hline \multicolumn{7}{|l|}{ Need permission for medical help } \\
\hline Big problem & 0.019 & 0.172 & $1.020(0.728-1.427)$ & -0.070 & 0.178 & $0.932(0.658-1.321)$ \\
\hline \multicolumn{7}{|l|}{ Not a big problem (reference) } \\
\hline \multicolumn{7}{|l|}{ Need money for medical help } \\
\hline Big problem & 0.092 & 0.221 & $1.097(0.711-1.691)$ & -0.204 & 0.225 & $0.816(0.525-1.267)$ \\
\hline \multicolumn{7}{|l|}{ Not a big problem (reference) } \\
\hline \multicolumn{7}{|l|}{ Distance to health facility } \\
\hline Big problem & -0.001 & 0.117 & $0.999(0.794-1.256)$ & $0.214^{*}$ & 0.121 & $1.239(0.977-1.572)$ \\
\hline \multicolumn{7}{|l|}{ Not a big problem (reference) } \\
\hline \multicolumn{7}{|c|}{ Do not want to visit health facility alone } \\
\hline Big problem & $-0.279^{*}$ & 0.145 & $0.756(0.569-1.005)$ & -0.030 & 0.146 & $0.971(0.729-1.292)$ \\
\hline \multicolumn{7}{|l|}{ Not a big problem (reference) } \\
\hline \multicolumn{7}{|c|}{ Heard family planning on radio last few months } \\
\hline No & 0.166 & 0.118 & $1.180(0.937-1.487)$ & $0.416^{\star \star \star}$ & 0.122 & $1.515(1.193-1.925)$ \\
\hline \multicolumn{7}{|l|}{ Yes (reference) } \\
\hline \multicolumn{7}{|c|}{ Heard family planning on TV last few months } \\
\hline No & 0.086 & 0.128 & $1.090(0.848-1.399)$ & -0.161 & 0.133 & $0.852(0.656-1.105)$ \\
\hline \multicolumn{7}{|l|}{ Yes (reference) } \\
\hline \multicolumn{7}{|c|}{ Heard family planning in newspaper/magazine last few months } \\
\hline No & $0.550 * \star$ & 0.260 & $1.733(1.042-2.883)$ & -0.072 & 0.234 & $0.930(0.588-1.473)$ \\
\hline \multicolumn{7}{|l|}{ Yes (reference) } \\
\hline \multicolumn{7}{|l|}{ REGRESSION STATISTICS } \\
\hline N observations & & 4142 & & & & \\
\hline -2 LL Ratio (Final) & & $2013.318^{\star \star \star}$ & & & & \\
\hline Chi- Square (Pearson) & & 16475.971 & & & & \\
\hline Pseudo R Squared (McFadden) & & 0.162 & & & & \\
\hline
\end{tabular}


Odds ratio maternal health: Ghana (continued)

\begin{tabular}{|c|c|c|c|c|c|}
\hline \multicolumn{3}{|c|}{ Poor access } & \multicolumn{3}{|c|}{ Low access } \\
\hline B & S.E. & Exp B $(95 \%$ Cl) & B & S.E. & Exp B $(95 \%$ Cl) \\
\hline $0.359 * *$ & 0.173 & $1.431(1.02-2.008)$ & -0.072 & 0.230 & $0.93(0.592-1.462)$ \\
\hline-0.174 & 0.233 & $0.84(0.532-1.328)$ & $-0.739 * *$ & 0.374 & $0.478(0.23-0.993)$ \\
\hline-0.118 & 0.127 & $0.889(0.693-1.14)$ & $-0.404^{\star *}$ & 0.161 & $0.668(0.487-0.916)$ \\
\hline 0.095 & 0.145 & $1.099(0.827-1.461)$ & 0.274 & 0.194 & $1.315(0.899-1.924)$ \\
\hline 0.193 & 0.127 & $1.212(0.946-1.554)$ & $0.273^{*}$ & 0.156 & $1.314(0.968-1.784)$ \\
\hline-0.080 & 0.138 & $0.923(0.704-1.21)$ & -0.258 & 0.170 & $0.773(0.554-1.077)$ \\
\hline 0.178 & 0.339 & $1.195(0.614-2.324)$ & 0.423 & 0.264 & $1.526(0.909-2.562)$ \\
\hline
\end{tabular}


Description of data: Odds ratio maternal health: Nigeria

\begin{tabular}{|c|c|c|c|c|c|c|}
\hline \multirow{2}{*}{ Background characteristics } & \multicolumn{3}{|c|}{ High access } & \multicolumn{3}{|c|}{ Middle access } \\
\hline & B & S.E. & $\operatorname{Exp} B(95 \% \mathrm{Cl})$ & B & S.E. & $\operatorname{Exp} B(95 \% \mathrm{Cl})$ \\
\hline \multicolumn{7}{|l|}{ Higher access } \\
\hline Intercept & -0.152 & 0.340 & & $1.011^{\star \star \star}$ & 0.297 & \\
\hline Maternal age & $-0.023^{\star \star *}$ & 0.007 & $0.977(0.963-0.992)$ & $-0.020 * \star \star$ & 0.007 & $0.98(0.967-0.994)$ \\
\hline Number of children alive & $0.096^{\star \star \star}$ & 0.027 & $1.101(1.045-1.16)$ & $0.084^{* * *}$ & 0.026 & $1.087(1.033-1.145)$ \\
\hline \multicolumn{7}{|l|}{ Marital status } \\
\hline Never married & 0.078 & 0.206 & $1.081(0.722-1.619)$ & -0.112 & 0.203 & $0.894(0.6-1.332)$ \\
\hline \multicolumn{6}{|l|}{ Married (reference) } & $1.055(0.729-1.527)$ \\
\hline \multicolumn{7}{|l|}{ Maternal Education } \\
\hline No education & 0.135 & 0.122 & $1.145(0.902-1.454)$ & $-0.371^{\star \star \star}$ & 0.141 & $0.69(0.524-0.909)$ \\
\hline Primary & 0.034 & 0.097 & $1.034(0.855-1.251)$ & -0.082 & 0.096 & $0.921(0.763-1.111)$ \\
\hline \multicolumn{7}{|l|}{ Secondary/ Higher (reference) } \\
\hline \multicolumn{7}{|l|}{ Ethnicity } \\
\hline Other minorities & $-0.614^{* * *}$ & 0.139 & $0.541(0.412-0.71)$ & -0.013 & 0.120 & $0.987(0.78-1.249)$ \\
\hline Fulani & -0.385 & 0.265 & $0.681(0.405-1.143)$ & -0.142 & 0.395 & $0.868(0.4-1.883)$ \\
\hline Igbo & -0.276 & 0.194 & $0.759(0.519-1.109)$ & $0.592^{\star \star \star}$ & 0.154 & $1.808(1.336-2.447)$ \\
\hline Hausa & -0.149 & 0.193 & $0.862(0.59-1.259)$ & 0.163 & 0.223 & $1.177(0.76-1.822)$ \\
\hline \multicolumn{7}{|l|}{ Yoruba (reference) } \\
\hline \multicolumn{7}{|l|}{ Maternal Occupation } \\
\hline Not working & 0.051 & 0.154 & $1.052(0.779-1.421)$ & 0.093 & 0.132 & $1.097(0.847-1.422)$ \\
\hline Services and manual & $0.400 * * *$ & 0.151 & $1.492(1.109-2.006)$ & 0.195 & 0.131 & $1.215(0.94-1.57)$ \\
\hline Sales & $0.254^{\star}$ & 0.140 & $1.289(0.979-1.698)$ & $0.262^{\star \star}$ & 0.118 & $1.3(1.032-1.638)$ \\
\hline Agriculture & $0.529 * \star \star$ & 0.183 & $1.697(1.187-2.428)$ & 0.261 & 0.174 & $1.298(0.922-1.826)$ \\
\hline \multicolumn{7}{|l|}{ White collar (reference) } \\
\hline \multicolumn{7}{|l|}{ Household Wealth } \\
\hline Poorest & $1.553^{\star \star \star}$ & 0.235 & $4.726(2.982-7.489)$ & 0.426 & 0.297 & $1.531(0.855-2.74)$ \\
\hline Poorer & $1.012^{\star \star \star}$ & 0.158 & $2.75(2.018-3.748)$ & 0.099 & 0.167 & $1.104(0.796-1.533)$ \\
\hline Middle & $0.604^{\star * \star}$ & 0.124 & $1.829(1.433-2.334)$ & -0.184 & 0.121 & $0.832(0.657-1.054)$ \\
\hline Richer & $0.390 * * \star$ & 0.097 & $1.476(1.221-1.785)$ & $-0.310 * \star \star$ & 0.089 & $0.733(0.616-0.873)$ \\
\hline \multicolumn{7}{|l|}{ Richest (reference) } \\
\hline \multicolumn{7}{|l|}{ Residence } \\
\hline Rural & $-0.424^{\star \star \star}$ & 0.089 & $0.654(0.55-0.779)$ & 0.083 & 0.088 & $1.086(0.914-1.29)$ \\
\hline \multicolumn{7}{|l|}{ Urban (reference) } \\
\hline \multicolumn{7}{|l|}{ Region } \\
\hline North Central & $-0.974 * \star \star$ & 0.142 & $0.377(0.286-0.499)$ & $-1.093^{\star \star \star}$ & 0.121 & $0.335(0.264-0.425)$ \\
\hline North East & $-0.851^{\star \star \star}$ & 0.176 & $0.427(0.302-0.603)$ & $-2.791^{\star \star \star}$ & 0.226 & $0.061(0.039-0.096)$ \\
\hline North West & $-1.582^{\star \star \star}$ & 0.179 & $0.206(0.145-0.292)$ & $-3.498^{\star \star \star}$ & 0.217 & $0.03(0.02-0.046)$ \\
\hline South East & 0.107 & 0.211 & $1.112(0.736-1.681)$ & -0.259 & 0.169 & $0.771(0.554-1.073)$ \\
\hline South South & 0.031 & 0.151 & $1.031(0.767-1.387)$ & $-1.143^{\star \star *}$ & 0.136 & $0.319(0.244-0.417)$ \\
\hline \multicolumn{7}{|l|}{ South West (reference) } \\
\hline Has health insurance & & & & & & \\
\hline No & 0.155 & 0.212 & $1.167(0.77-1.77)$ & -0.237 & 0.163 & $0.789(0.574-1.085)$ \\
\hline Yes (reference) & & & & & & \\
\hline Religion & & & & & & \\
\hline Catholic & $0.218^{*}$ & 0.127 & $1.244(0.97-1.595)$ & $0.459 * \star \star$ & 0.113 & $1.582(1.269-1.973)$ \\
\hline Traditionalist & -0.441 & 0.484 & $0.643(0.249-1.661)$ & -0.755 & 0.535 & $0.47(0.165-1.34)$ \\
\hline Islam & -0.008 & 0.103 & $0.992(0.81-1.214)$ & -0.087 & 0.095 & $0.916(0.76-1.104)$ \\
\hline Other Christian (reference) & & & & & & \\
\hline Need permission for medical he & & & & & & \\
\hline Big problem & -0.010 & 0.171 & $0.99(0.709-1.384)$ & -0.248 & 0.188 & $0.781(0.54-1.128)$ \\
\hline Not a big problem (reference) & & & & & & \\
\hline Need money for medical help & & & & & & \\
\hline Big problem & -0.004 & 0.086 & $0.996(0.843-1.178)$ & -0.129 & 0.085 & $0.879(0.744-1.037)$ \\
\hline Not a big problem (reference) & & & & & & \\
\hline
\end{tabular}


Odds ratio maternal health: Nigeria (continued)

\begin{tabular}{|c|c|c|c|c|c|}
\hline \multicolumn{3}{|c|}{ Poor access } & \multicolumn{3}{|l|}{ Low access } \\
\hline B & S.E. & Exp B $(95 \% \mathrm{Cl})$ & B & S.E. & Exp B (95\% Cl) \\
\hline$-1.743^{\star \star \star}$ & 0.416 & & $-1.539 * \star$ & 0.693 & \\
\hline$-0.036^{\star \star \star}$ & 0.008 & $0.965(0.95-0.98)$ & $-0.045^{\star \star \star}$ & 0.014 & $0.956(0.93-0.982)$ \\
\hline $0.118^{\star * *}$ & 0.027 & $1.126(1.067-1.188)$ & $0.107^{\star \star}$ & 0.047 & $1.113(1.016-1.22)$ \\
\hline 0.324 & 0.207 & $1.382(0.922-2.072)$ & 0.438 & 0.423 & $1.55(0.676-3.551)$ \\
\hline 0.081 & 0.195 & $1.084(0.74-1.588)$ & 0.222 & 0.346 & $1.248(0.634-2.459)$ \\
\hline $0.358^{\star * *}$ & 0.120 & $1.431(1.132-1.809)$ & $0.615^{\star * *}$ & 0.201 & $1.849(1.247-2.742)$ \\
\hline $0.327^{\star \star \star}$ & 0.100 & $1.387(1.14-1.687)$ & $0.58^{\star \star *}$ & 0.183 & $1.786(1.247-2.557)$ \\
\hline-0.203 & 0.155 & $0.816(0.603-1.105)$ & 0.201 & 0.301 & $1.223(0.677-2.208)$ \\
\hline-0.127 & 0.260 & $0.881(0.529-1.466)$ & -0.109 & 0.443 & $0.897(0.376-2.139)$ \\
\hline$-0.703^{* \star *}$ & 0.247 & $0.495(0.305-0.803)$ & 0.014 & 0.504 & $1.014(0.378-2.722)$ \\
\hline $0.348^{*}$ & 0.196 & $1.416(0.965-2.078)$ & $0.803^{\star \star}$ & 0.351 & $2.231(1.122-4.438)$ \\
\hline $0.457^{\star \star}$ & 0.193 & $1.579(1.081-2.307)$ & 0.562 & 0.432 & $1.754(0.752-4.093)$ \\
\hline $0.542^{\star \star \star}$ & 0.196 & $1.719(1.172-2.522)$ & $0.885^{\star \star}$ & 0.435 & $2.422(1.032-5.686)$ \\
\hline $0.694^{\star \star \star}$ & 0.184 & $2.001(1.395-2.871)$ & $1.004^{\star \star}$ & 0.420 & $2.729(1.198-6.219)$ \\
\hline $0.511^{\star \star}$ & 0.224 & $1.667(1.076-2.584)$ & $1.448^{\star \star \star}$ & 0.456 & $4.253(1.741-10.392)$ \\
\hline $1.886^{\star \star *}$ & 0.224 & $6.592(4.247-10.233)$ & $1.173^{* \star *}$ & 0.337 & $3.23(1.668-6.255)$ \\
\hline $0.879 * \star \star$ & 0.163 & $2.408(1.75-3.312)$ & $0.458^{*}$ & 0.275 & $1.58(0.921-2.711)$ \\
\hline $0.593^{\star \star \star}$ & 0.131 & $1.81(1.401-2.338)$ & 0.237 & 0.238 & $1.268(0.795-2.023)$ \\
\hline $0.213^{\star \star}$ & 0.105 & $1.237(1.006-1.522)$ & $-0.408^{\star}$ & 0.219 & $0.665(0.433-1.022)$ \\
\hline$-0.202^{\star \star}$ & 0.095 & $0.817(0.678-0.985)$ & $-0.618^{\star \star \star}$ & 0.173 & $0.539(0.384-0.757)$ \\
\hline 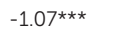 & 0.161 & $0.343(0.25-0.47)$ & -0.406 & 0.286 & $0.666(0.381-1.166)$ \\
\hline$-0.517^{\star \star \star *}$ & 0.181 & $0.596(0.418-0.85)$ & 0.150 & 0.311 & $1.162(0.632-2.136)$ \\
\hline$-1.063^{\star \star \star}$ & 0.181 & $0.346(0.242-0.492)$ & $-1.068^{\star \star \star}$ & 0.337 & $0.344(0.178-0.665)$ \\
\hline-0.040 & 0.269 & $0.961(0.567-1.629)$ & 0.538 & 0.506 & $1.712(0.635-4.618)$ \\
\hline $0.337^{* *}$ & 0.163 & $1.401(1.019-1.928)$ & $-1.394^{\star \star *}$ & 0.431 & $0.248(0.107-0.577)$ \\
\hline $0.451^{*}$ & 0.269 & $1.57(0.926-2.661)$ & -0.574 & 0.367 & $0.563(0.274-1.156)$ \\
\hline-0.049 & 0.150 & $0.952(0.709-1.278)$ & 0.304 & 0.239 & $1.355(0.847-2.166)$ \\
\hline-0.755 & 0.568 & $0.47(0.154-1.432)$ & -0.970 & 1.235 & $0.379(0.034-4.263)$ \\
\hline-0.025 & 0.112 & $0.976(0.783-1.215)$ & $0.357^{*}$ & 0.197 & $1.429(0.971-2.105)$ \\
\hline 0.016 & 0.169 & $1.016(0.73-1.415)$ & -0.477 & 0.350 & $0.62(0.312-1.232)$ \\
\hline $0.266^{\star \star \star *}$ & 0.087 & $1.304(1.1-1.547)$ & 0.032 & 0.151 & $1.033(0.768-1.389)$ \\
\hline
\end{tabular}


Odds ratio maternal health: Nigeria (continued)

\begin{tabular}{|c|c|c|c|c|c|c|}
\hline \multirow{2}{*}{ Background characteristics } & \multicolumn{3}{|l|}{ High access } & \multicolumn{3}{|c|}{ Middle access } \\
\hline & $\bar{B}$ & S.E. & $\operatorname{Exp} \mathrm{B}(95 \% \mathrm{Cl})$ & B & S.E. & $\operatorname{Exp} B(95 \% \mathrm{Cl})$ \\
\hline \multicolumn{7}{|l|}{ Distance to health facility } \\
\hline Big problem & $0.192^{*}$ & 0.111 & $1.211(0.974-1.506)$ & $0.502^{\star \star *}$ & 0.112 & $1.652(1.327-2.057$ \\
\hline \multicolumn{7}{|l|}{ Not a big problem (reference) } \\
\hline \multicolumn{7}{|c|}{ Do not want to visit health facility alone } \\
\hline Big problem & -0.140 & 0.163 & $0.869(0.632-1.196)$ & -0.018 & 0.163 & $0.982(0.714-1.351$ \\
\hline \multicolumn{7}{|l|}{ Not a big problem (reference) } \\
\hline \multicolumn{7}{|l|}{ Attitude of the health workers } \\
\hline Big problem & 0.008 & 0.120 & $1.008(0.797-1.275)$ & $-0.254^{\star *}$ & 0.121 & $0.776(0.612-0.983$ \\
\hline \multicolumn{7}{|l|}{ Not a big problem (reference) } \\
\hline \multicolumn{7}{|c|}{ Heard family planning on radio last few months } \\
\hline No & -0.087 & 0.090 & $0.917(0.768-1.095)$ & -0.068 & 0.089 & $0.934(0.785-1.112$ \\
\hline \multicolumn{7}{|l|}{ Yes (reference) } \\
\hline \multicolumn{7}{|c|}{ Heard family planning on TV last few months } \\
\hline No & -0.150 & 0.100 & $0.861(0.708-1.047)$ & -0.132 & 0.094 & $0.877(0.729-1.053$ \\
\hline \multicolumn{7}{|l|}{ Yes (reference) } \\
\hline \multicolumn{7}{|c|}{ Heard family planning in print last few months } \\
\hline No & $0.535^{\star * *}$ & 0.134 & $1.707(1.314-2.217)$ & $0.308^{* * *}$ & 0.109 & $1.361(1.098-1.686$ \\
\hline \multicolumn{7}{|l|}{ Yes (reference) } \\
\hline \multicolumn{7}{|l|}{ REGRESSION STATISTICS } \\
\hline N observations & 7725 & & & & & \\
\hline-2 LL Ratio (Final) & $3055.958^{* \star *}$ & & & & & \\
\hline Chi-Square (Pearson) & 29784.704 & & & & & \\
\hline Pseudo R Squared (McFadden) & 0.138 & & & & & \\
\hline
\end{tabular}

${ }^{* * *} p \leq 0.01 ;{ }^{* \star} p \leq 0.05 ;{ }^{*} p \leq 0.10$ (two-tailed test of significance) 
Odds ratio maternal health: Nigeria (continued)

\begin{tabular}{|c|c|c|c|c|c|}
\hline \multicolumn{3}{|c|}{ Poor access } & \multicolumn{3}{|c|}{ Low access } \\
\hline B & S.E. & Exp B $(95 \% \mathrm{Cl})$ & B & S.E. & Exp B $(95 \% \mathrm{Cl})$ \\
\hline $0.412^{\star \star \star}$ & 0.111 & $1.51(1.216-1.876)$ & 0.104 & 0.193 & $1.109(0.76-1.62)$ \\
\hline-0.235 & 0.171 & $0.791(0.566-1.106)$ & -0.153 & 0.306 & $0.859(0.471-1.565)$ \\
\hline$-0.605^{\star \star *}$ & 0.135 & $0.546(0.419-0.712)$ & $-0.871^{\star \star *}$ & 0.284 & $0.418(0.24-0.73)$ \\
\hline 0.072 & 0.093 & $1.075(0.896-1.29)$ & -0.075 & 0.166 & $0.928(0.67-1.286)$ \\
\hline 0.065 & 0.110 & $1.068(0.861-1.324)$ & 0.325 & 0.228 & $1.384(0.885-2.165)$ \\
\hline 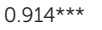 & 0.181 & $2.495(1.748-3.56)$ & -0.212 & 0.299 & $0.809(0.45-1.455)$ \\
\hline
\end{tabular}





\section{APPENDIX C}

Additional information for Chapter 4 


\section{Appendix C1 Definition of indicators used in the analysis, Ghana}

Description of data: Grouping description of outcome variable

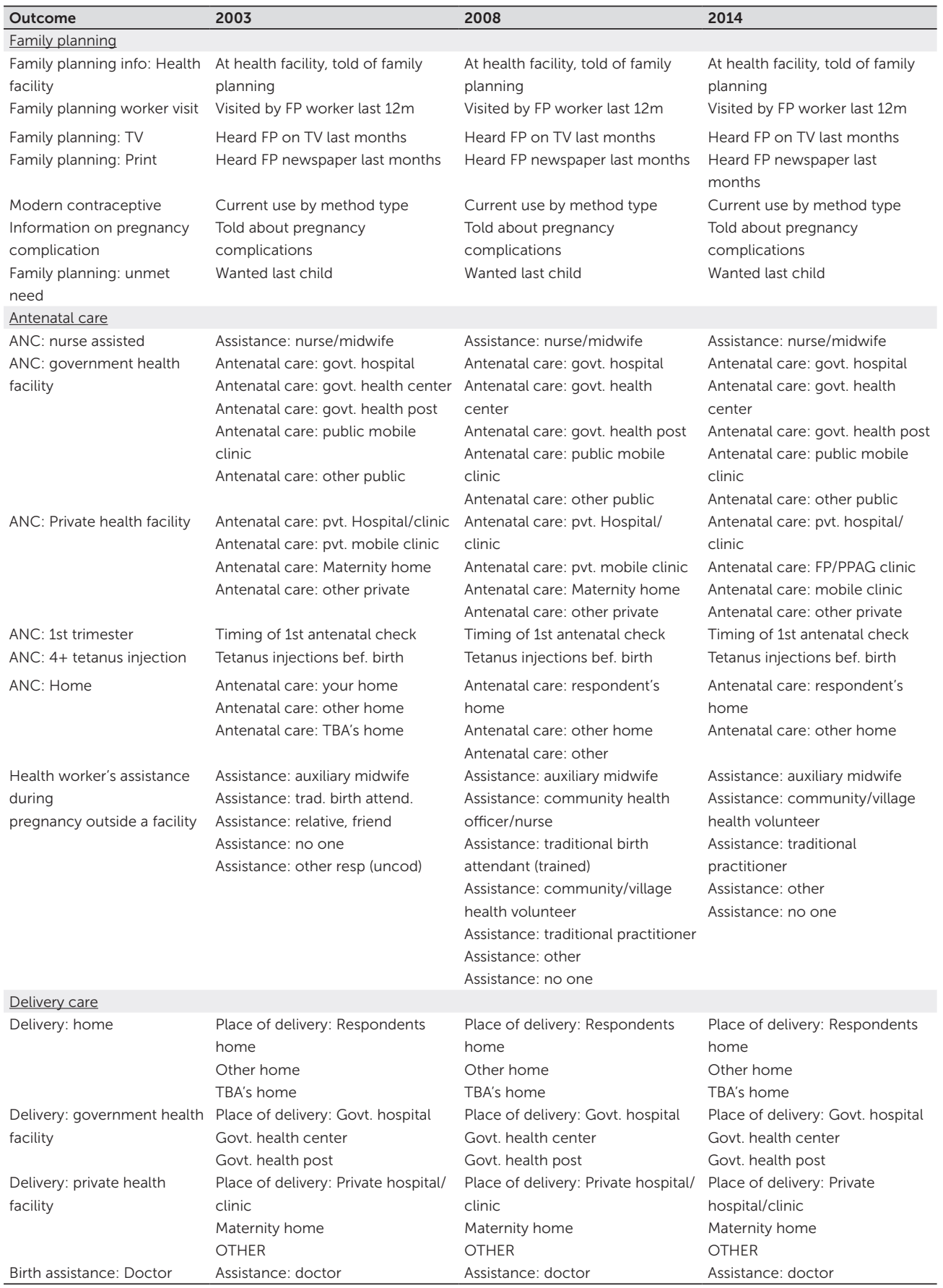




\begin{tabular}{|c|c|c|c|}
\hline Outcome & 2003 & 2008 & 2013 \\
\hline \multicolumn{4}{|l|}{ Family planning } \\
\hline $\begin{array}{l}\text { Family planning info: Health } \\
\text { facility }\end{array}$ & $\begin{array}{l}\text { At health facility, told of family } \\
\text { planning }\end{array}$ & $\begin{array}{l}\text { At health facility, told of family } \\
\text { planning }\end{array}$ & $\begin{array}{l}\text { At health facility, told of family } \\
\text { planning }\end{array}$ \\
\hline Family planning worker visit & Visited by FP worker last $12 \mathrm{~m}$ & Visited by FP worker last $12 \mathrm{~m}$ & Visited by FP worker last $12 \mathrm{~m}$ \\
\hline Family planning: TV & Heard FP on TV last months & Heard FP on TV last months & Heard FP on TV last months \\
\hline Family planning: Print & $\begin{array}{l}\text { Heard FP newspaper last } \\
\text { months }\end{array}$ & Heard FP newspaper last months & Heard FP newspaper last months \\
\hline Modern contraceptive & Current use by method type & Current use by method type & Current use by method type \\
\hline $\begin{array}{l}\text { Information on pregnancy } \\
\text { complication }\end{array}$ & $\begin{array}{l}\text { Told about pregnancy } \\
\text { complications }\end{array}$ & $\begin{array}{l}\text { Told about pregnancy } \\
\text { complications }\end{array}$ & $\begin{array}{l}\text { Told about pregnancy } \\
\text { complications }\end{array}$ \\
\hline Family planning: unmet need & Wanted last child & Wanted last child & Wanted last child \\
\hline \multicolumn{4}{|l|}{ Antenatal care } \\
\hline $\begin{array}{l}\text { ANC: nurse assisted } \\
\text { ANC: government health } \\
\text { facility }\end{array}$ & $\begin{array}{l}\text { Assistance: nurse/midwife } \\
\text { Antenatal care: govt. hospital } \\
\text { Antenatal care: govt. health } \\
\text { center } \\
\text { Antenatal care: govt. health } \\
\text { post } \\
\text { Antenatal care: public mobile } \\
\text { clinic } \\
\text { Antenatal care: other public }\end{array}$ & $\begin{array}{l}\text { Assistance: nurse/midwife } \\
\text { Antenatal care: govt. hospital } \\
\text { Antenatal care: govt. health center } \\
\text { Antenatal care: govt. health post/ } \\
\text { dispensary } \\
\text { Antenatal care: other public }\end{array}$ & $\begin{array}{l}\text { Assistance: nurse/midwife } \\
\text { Antenatal care: government } \\
\text { hospital } \\
\text { Antenatal care: government health } \\
\text { center } \\
\text { Antenatal care: government health } \\
\text { post/ dispensary } \\
\text { Antenatal care: other public sector }\end{array}$ \\
\hline ANC: Private health facility & $\begin{array}{l}\text { Antenatal care: pvt. hospital/ } \\
\text { clinic } \\
\text { Antenatal care: other private }\end{array}$ & $\begin{array}{l}\text { Antenatal care: pvt. hospital/clinic } \\
\text { Antenatal care: other private }\end{array}$ & $\begin{array}{l}\text { Antenatal care: private hospital/ } \\
\text { clinic } \\
\text { Antenatal care: other private } \\
\text { medical sector }\end{array}$ \\
\hline ANC: 1st trimester & Timing of 1st antenatal check & Timing of 1st antenatal check & Timing of 1st antenatal check \\
\hline ANC: $4+$ tetanus injection & Tetanus injections bef. birth & Tetanus injections bef. birth & Tetanus injections bef. birth \\
\hline ANC: Home & $\begin{array}{l}\text { Antenatal care: your home } \\
\text { Antenatal care: other home } \\
\text { Antenatal care: other }\end{array}$ & $\begin{array}{l}\text { Antenatal care: your home } \\
\text { Antenatal care: other home } \\
\text { Antenatal care: other }\end{array}$ & $\begin{array}{l}\text { Antenatal care: respondent's home } \\
\text { Antenatal care: other home } \\
\text { Antenatal care: other }\end{array}$ \\
\hline $\begin{array}{l}\text { Health worker's assistance } \\
\text { during } \\
\text { pregnancy outside a facility }\end{array}$ & $\begin{array}{l}\text { Assistance: auxiliary midwife } \\
\text { Assistance: trad.birth attend. } \\
\text { Assistance: relative, friend } \\
\text { Assistance: other resp (uncod) } \\
\text { Assistance: no one }\end{array}$ & $\begin{array}{l}\text { Assistance: auxiliary midwife } \\
\text { Assistance: trad.birth attend. } \\
\text { Assistance: relative, friend } \\
\text { Assistance: other resp (uncod) } \\
\text { Assistance: no one. }\end{array}$ & $\begin{array}{l}\text { Assistance: auxiliary midwife } \\
\text { Assistance: community extension } \\
\text { health worker } \\
\text { Assistance: traditional birth } \\
\text { attendant } \\
\text { Assistance: relative/ friend } \\
\text { Assistance: other Assistance: no } \\
\text { one }\end{array}$ \\
\hline \multicolumn{4}{|l|}{ Delivery care } \\
\hline Delivery: home & $\begin{array}{l}\text { Place of delivery: Respondents } \\
\text { home } \\
\text { Other home } \\
\text { TBA's home }\end{array}$ & $\begin{array}{l}\text { Place of delivery: Respondents } \\
\text { home } \\
\text { Other home } \\
\text { TBA's home }\end{array}$ & $\begin{array}{l}\text { Place of delivery: Respondents } \\
\text { home } \\
\text { Other home } \\
\text { TBA's home }\end{array}$ \\
\hline $\begin{array}{l}\text { Delivery: government health } \\
\text { facility }\end{array}$ & $\begin{array}{l}\text { Place of delivery: Govt. hospital } \\
\text { Govt. health center } \\
\text { Govt. health post }\end{array}$ & $\begin{array}{l}\text { Place of delivery: Govt. hospital } \\
\text { Govt. health center } \\
\text { Govt. health post }\end{array}$ & $\begin{array}{l}\text { Place of delivery: Govt. hospital } \\
\text { Govt. health center } \\
\text { Govt. health post }\end{array}$ \\
\hline Delivery: private health facility & $\begin{array}{l}\text { Place of delivery: Private hosp/ } \\
\text { clinic } \\
\text { Maternity home } \\
\text { OTHER }\end{array}$ & $\begin{array}{l}\text { Place of delivery: Private hosp/ } \\
\text { clinic } \\
\text { Maternity home } \\
\text { OTHER }\end{array}$ & $\begin{array}{l}\text { Place of delivery: Private hosp/ } \\
\text { clinic } \\
\text { Maternity home } \\
\text { OTHER }\end{array}$ \\
\hline Birth assistance: Doctor & Assistance: doctor & Assistance: doctor & Assistance: doctor \\
\hline
\end{tabular}




\section{Appendix C2 Definition of independent variables used in the analysis}

Description of data: Coding of the dependent and control variables

\begin{tabular}{|c|c|c|}
\hline Independent variable & Questions asked & Coding \\
\hline Age group & Age 5-year groups & Categorical \\
\hline \multirow[t]{2}{*}{$15-24$} & $15-19$ & \\
\hline & $20-24$ & \\
\hline \multirow[t]{5}{*}{$25-49$} & $25-29$ & \\
\hline & $30-34$ & \\
\hline & $35-39$ & \\
\hline & $40-44$ & \\
\hline & $45-49$ & \\
\hline Marital status & Current marital status & Categorical \\
\hline never & Never married & \\
\hline \multirow[t]{5}{*}{ now / then } & Married & \\
\hline & Living together & \\
\hline & Widowed & \\
\hline & Divorced & \\
\hline & Not living together & \\
\hline Maternal occupation & Respondent's occupation & Categorical \\
\hline not working & Not working & \\
\hline \multirow[t]{3}{*}{ Professional / sales } & Professional/technical/managerial & \\
\hline & Clerical & \\
\hline & Sales & \\
\hline \multirow[t]{2}{*}{ Agriculture } & Agricultural - self employed & \\
\hline & Agricultural - employee & \\
\hline \multirow[t]{4}{*}{ Others } & Household and domestic & \\
\hline & Services & \\
\hline & Skilled manual & \\
\hline & Unskilled manual & \\
\hline Location & Type of place of residence & Categorical \\
\hline \multicolumn{3}{|l|}{ urban } \\
\hline \multicolumn{3}{|l|}{ rural } \\
\hline Region (Ghana) & Region & Categorical \\
\hline Region (Nigeria) & Region & Categorical \\
\hline
\end{tabular}


Sociodemographic characteristics: Ghana (years 2000, 2005 \& 2014) and Nigeria (years 2003, 2008 \& 2013) Description of data: Distribution of the independent variables

\begin{tabular}{|c|c|c|c|c|c|c|}
\hline & GHANA & & & NIGERIA & & \\
\hline Maternal characteristic & 2003 & 2008 & 2014 & 2003 & 2008 & 2013 \\
\hline \multicolumn{7}{|l|}{ Age group } \\
\hline $25-49$ & $2128(76.6)$ & $1629(75.9)$ & $3371(78.5)$ & $2685(71.1)$ & $13249(73.5)$ & $15012(74.3)$ \\
\hline \multicolumn{7}{|l|}{ Marital status } \\
\hline Never & $87(3.1)$ & $117(5.4)$ & $363(8.5)$ & $103(2.7)$ & $455(2.5)$ & $538(2.7)$ \\
\hline \multicolumn{7}{|l|}{ Maternal occupation } \\
\hline Not working & $292(10.6)$ & $219(10.3)$ & 759 (17.7) & $1286(34.1)$ & $5704(31.8)$ & $5877(29.3)$ \\
\hline Professional / Sales & $689(25.0)$ & $753(35.3)$ & $1603(37.4)$ & $1556(41.2)$ & $6314(35.2)$ & $8498(42.3)$ \\
\hline Agriculture & $1260(45.7)$ & $812(38.0)$ & $1316(30.7)$ & $584(15.5)$ & 3508 (19.6) & $2458(12.2)$ \\
\hline Others & $519(18.8)$ & $351(16.4)$ & $608(14.2)$ & $349(9.2)$ & $2404(13.4)$ & $3240(16.1)$ \\
\hline \multicolumn{7}{|l|}{ Region (Ghana) } \\
\hline Western & $237(8.5)$ & $189(8.8)$ & $431(10.0)$ & - & - & - \\
\hline Central & $168(6.0)$ & $158(7.4)$ & $436(10.2)$ & - & - & - \\
\hline Greater Accra & $264(9.5)$ & $210(9.8)$ & $354(8.2)$ & - & - & - \\
\hline Volta & $202(7.3)$ & $181(8.4)$ & $346(8.1)$ & - & - & - \\
\hline Eastern & $228(8.2)$ & $187(8.7)$ & $397(9.2)$ & - & - & - \\
\hline Ashanti & $423(15.2)$ & $318(14.8)$ & $420(9.8)$ & - & - & - \\
\hline Brong Ahafo & $337(12.1)$ & $207(9.6)$ & $490(11.4)$ & - & - & - \\
\hline Northern & $429(15.4)$ & $306(14.3)$ & $622(14.5)$ & - & - & - \\
\hline Upper West & $265(9.5)$ & $181(8.4)$ & $434(10.1)$ & - & - & - \\
\hline South South & - & - & - & $380(10.1)$ & $2101(11.7)$ & $2500(12.4)$ \\
\hline South West & - & - & - & 429 (11.4) & $2263(12.6)$ & $2666(13.2)$ \\
\hline Total & 2777 & 2147 & 4294 & 3775 & 18028 & 20192 \\
\hline
\end{tabular}




\section{Appendix C3 Distribution of outcome variables (poorest $20 \%$, richest $20 \%$ and total sample size)}

Description of data: Distribution of the control variables, Ghana

\begin{tabular}{|c|c|c|c|c|c|c|c|c|c|}
\hline \multirow[t]{2}{*}{ Ghana } & & \multicolumn{5}{|c|}{2003} & \multirow[b]{2}{*}{ Poorest } & \multirow[b]{2}{*}{$\%$} & \multirow[b]{2}{*}{ Richest } \\
\hline & & Poorest & $\%$ & Richest & $\%$ & All women & & & \\
\hline Family planning info: Health & No & 141 & 18.98 & 178 & 23.96 & 743 & 115 & 15.13 & 190 \\
\hline \multirow[t]{2}{*}{ facility } & Yes & 147 & 18.8 & 183 & 23.4 & 782 & 116 & 20.03 & 123 \\
\hline & Total & 288 & 18.89 & 361 & 23.67 & 1525 & 231 & 17.25 & 313 \\
\hline \multirow[t]{3}{*}{ Family planning worker visit } & No & 492 & 20.95 & 482 & 20.52 & 2349 & 350 & 19.73 & 363 \\
\hline & Yes & 80 & 20.36 & 63 & 16.03 & 393 & 73 & 20.8 & 68 \\
\hline & Total & 572 & 20.86 & 545 & 19.88 & 2742 & 423 & 19.91 & 431 \\
\hline \multirow[t]{3}{*}{ FP Info: TV } & No & 507 & 29.02 & 98 & 5.61 & 1747 & 401 & 28.64 & 90 \\
\hline & Yes & 67 & 6.71 & 447 & 44.79 & 998 & 23 & 3.17 & 341 \\
\hline & Total & 574 & 20.91 & 545 & 19.85 & 2745 & 424 & 19.94 & 431 \\
\hline \multirow[t]{3}{*}{ FP Info: Print } & No & 567 & 23.29 & 350 & 14.38 & 2434 & 420 & 21.19 & 350 \\
\hline & Yes & 6 & 1.95 & 195 & 63.31 & 308 & 4 & 2.78 & 81 \\
\hline & Total & 573 & 20.9 & 545 & 19.88 & 2742 & 424 & 19.94 & 431 \\
\hline \multirow[t]{3}{*}{ Modern contraceptive } & No & 23 & 13.07 & 52 & 29.55 & 176 & 5 & 4.2 & 43 \\
\hline & Yes & 66 & 12.87 & 158 & 30.8 & 513 & 58 & 15.59 & 98 \\
\hline & Total & 89 & 12.92 & 210 & 30.48 & 689 & 63 & 12.83 & 141 \\
\hline Information on pregnancy & No & 251 & 24.25 & 152 & 14.69 & 1035 & 177 & 27.87 & 93 \\
\hline \multirow[t]{2}{*}{ complication } & Yes & 239 & 16.07 & 385 & 25.89 & 1487 & 213 & 15.24 & 331 \\
\hline & Total & 490 & 19.43 & 537 & 21.29 & 2522 & 390 & 19.18 & 424 \\
\hline \multirow[t]{3}{*}{ Family planning: unmet need } & No & 374 & 22.89 & 337 & 20.62 & 1634 & 290 & 21.9 & 287 \\
\hline & Yes & 197 & 17.91 & 207 & 18.82 & 1100 & 133 & 16.58 & 144 \\
\hline & Total & 571 & 20.89 & 544 & 19.9 & 2734 & 423 & 19.9 & 431 \\
\hline \multirow{3}{*}{$\begin{array}{l}\text { Health worker's assistance } \\
\text { during pregnancy outside } \\
\text { a facility }\end{array}$} & No & 110 & 9.81 & 445 & 39.7 & 1121 & 232 & 16.84 & 365 \\
\hline & Yes & 461 & 28.6 & 99 & 6.14 & 1612 & 190 & 25.47 & 66 \\
\hline & Total & 571 & 20.89 & 544 & 19.9 & 2733 & 422 & 19.87 & 431 \\
\hline \multirow[t]{3}{*}{ ANC: nurse assisted } & No & 466 & 28.59 & 125 & 7.67 & 1630 & 338 & 31.35 & 86 \\
\hline & Yes & 105 & 9.52 & 419 & 37.99 & 1103 & 84 & 8.03 & 345 \\
\hline & Total & 571 & 20.89 & 544 & 19.9 & 2733 & 422 & 19.87 & 431 \\
\hline \multirow{3}{*}{$\begin{array}{l}\text { ANC: government health } \\
\text { facility }\end{array}$} & No & 45 & 15.46 & 87 & 29.9 & 291 & 19 & 8.37 & 81 \\
\hline & Yes & 446 & 19.96 & 451 & 20.19 & 2234 & 375 & 20.73 & 341 \\
\hline & Total & 491 & 19.45 & 538 & 21.31 & 2525 & 394 & 19.35 & 422 \\
\hline ANC: Private health facility & No & 460 & 20.6 & 431 & 19.3 & 2233 & 382 & 20.6 & 345 \\
\hline & Yes & 31 & 10.62 & 107 & 36.64 & 292 & 12 & 6.59 & 77 \\
\hline & Total & 491 & 19.45 & 538 & 21.31 & 2525 & 394 & 19.35 & 422 \\
\hline ANC: 1st trimester & No & 285 & 22.44 & 203 & 15.98 & 1270 & 208 & 24.21 & 126 \\
\hline & Yes & 204 & 16.36 & 334 & 26.78 & 1247 & 180 & 15.38 & 294 \\
\hline & Total & 489 & 19.43 & 537 & 21.33 & 2517 & 388 & 19.12 & 420 \\
\hline ANC: +4 tetanus injection & No & 145 & 33.56 & 30 & 6.94 & 432 & 83 & 32.17 & 18 \\
\hline & Yes & 421 & 18.67 & 493 & 21.86 & 2255 & 335 & 18.19 & 407 \\
\hline & Total & 566 & 21.06 & 523 & 19.46 & 2687 & 418 & 19.9 & 425 \\
\hline ANC: Home & No & 476 & 19.23 & 537 & 21.7 & 2475 & 384 & 19.26 & 419 \\
\hline & Yes & 15 & 30 & 1 & 2 & 50 & 10 & 23.81 & 3 \\
\hline & Total & 491 & 19.45 & 538 & 21.31 & 2525 & 394 & 19.35 & 422 \\
\hline Home delivery & No & 121 & 10.06 & 466 & 38.74 & 1203 & 108 & 8.88 & 384 \\
\hline & Yes & 453 & 29.38 & 79 & 5.12 & 1542 & 316 & 34.69 & 47 \\
\hline & Total & 574 & 20.91 & 545 & 19.85 & 2745 & 424 & 19.93 & 431 \\
\hline Delivery: government health & No & 470 & 26.18 & 184 & 10.25 & 1795 & 325 & 29.73 & 121 \\
\hline facility & Yes & 104 & 10.95 & 361 & 38 & 950 & 99 & 9.57 & 310 \\
\hline & Total & 574 & 20.91 & 545 & 19.85 & 2745 & 424 & 19.93 & 431 \\
\hline Delivery: private health & No & 560 & 22.37 & 441 & 17.62 & 2503 & 416 & 21.38 & 357 \\
\hline facility & Yes & 14 & 5.79 & 104 & 42.98 & 242 & 8 & 4.42 & 74 \\
\hline & Total & 574 & 20.91 & 545 & 19.85 & 2745 & 424 & 19.93 & 431 \\
\hline Birth assistance: Doctor & No & 556 & 21.77 & 442 & 17.31 & 2554 & 412 & 21.54 & 330 \\
\hline & Yes & 15 & 8.38 & 102 & 56.98 & 179 & 10 & 4.74 & 101 \\
\hline & Total & 571 & 20.89 & 544 & 19.9 & 2733 & 422 & 19.87 & 431 \\
\hline Caesarean section & No & 561 & 21.32 & 487 & 18.51 & 2631 & 415 & 20.85 & 373 \\
\hline & Yes & 10 & 10.00 & 56 & 56.00 & 100 & 9 & 6.67 & 57 \\
\hline & Total & 571 & 20.91 & 543 & 19.88 & 2731 & 424 & 19.95 & 430 \\
\hline
\end{tabular}


Distribution of the control variables, Ghana (continued)

\begin{tabular}{|c|c|c|c|c|c|c|}
\hline \multicolumn{4}{|l|}{2008} & \multicolumn{3}{|c|}{2014} \\
\hline$\%$ & All women & Poorest & $\%$ & Richest & $\%$ & All women \\
\hline 25 & 760 & 312 & 22.29 & 246 & 17.57 & 1400 \\
\hline 21.24 & 579 & 354 & 24.2 & 220 & 15.04 & 1463 \\
\hline 23.38 & 1339 & 666 & 23.26 & 466 & 16.28 & 2863 \\
\hline 20.46 & 1774 & 854 & 23.47 & 586 & 16.1 & 3639 \\
\hline 19.37 & 351 & 174 & 26.56 & 76 & 11.6 & 655 \\
\hline 20.28 & 2125 & 1028 & 23.94 & 662 & 15.42 & 4294 \\
\hline 6.43 & 1400 & 889 & 35.24 & 141 & 5.59 & 2523 \\
\hline 46.97 & 726 & 139 & 7.85 & 521 & 29.42 & 1771 \\
\hline 20.27 & 2126 & 1028 & 23.94 & 662 & 15.42 & 4294 \\
\hline 17.66 & 1982 & 1018 & 24.7 & 581 & 14.1 & 4121 \\
\hline 56.25 & 144 & 10 & 5.78 & 81 & 46.82 & 173 \\
\hline 20.27 & 2126 & 1028 & 23.94 & 662 & 15.42 & 4294 \\
\hline 36.13 & 119 & 22 & 15.17 & 50 & 34.48 & 145 \\
\hline 26.34 & 372 & 220 & 19.98 & 152 & 13.81 & 1101 \\
\hline 28.72 & 491 & 242 & 19.42 & 202 & 16.21 & 1246 \\
\hline 14.65 & 635 & 181 & 24.97 & 72 & 9.93 & 725 \\
\hline 23.68 & 1398 & 791 & 23.11 & 585 & 17.09 & 3423 \\
\hline 20.86 & 2033 & 972 & 23.43 & 657 & 15.84 & 4148 \\
\hline 21.68 & 1324 & 769 & 25.43 & 500 & 16.53 & 3024 \\
\hline 17.96 & 802 & 259 & 20.39 & 162 & 12.76 & 1270 \\
\hline 20.27 & 2126 & 1028 & 23.94 & 662 & 15.42 & 4294 \\
\hline 26.49 & 1378 & 783 & 21.82 & 624 & 17.39 & 3588 \\
\hline 8.85 & 746 & 245 & 34.7 & 38 & 5.38 & 706 \\
\hline 20.29 & 2124 & 1028 & 23.94 & 662 & 15.42 & 4294 \\
\hline 7.98 & 1078 & 541 & 34.31 & 124 & 7.86 & 1577 \\
\hline 32.98 & 1046 & 487 & 17.92 & 538 & 19.8 & 2717 \\
\hline 20.29 & 2124 & 1028 & 23.94 & 662 & 15.42 & 4294 \\
\hline 35.68 & 227 & 34 & 9.42 & 133 & 36.84 & 361 \\
\hline 18.85 & 1809 & 941 & 24.75 & 526 & 13.83 & 3802 \\
\hline 20.73 & 2036 & 975 & 23.42 & 659 & 15.83 & 4163 \\
\hline 18.61 & 1854 & 939 & 24.61 & 526 & 13.78 & 3816 \\
\hline 42.31 & 182 & 36 & 10.37 & 133 & 38.33 & 347 \\
\hline 20.73 & 2036 & 975 & 23.42 & 659 & 15.83 & 4163 \\
\hline 14.67 & 859 & 381 & 26.53 & 160 & 11.14 & 1436 \\
\hline 25.13 & 1170 & 593 & 21.76 & 499 & 18.31 & 2725 \\
\hline 20.7 & 2029 & 974 & 23.41 & 659 & 15.84 & 4161 \\
\hline 6.98 & 258 & 160 & 32.52 & 42 & 8.54 & 492 \\
\hline 22.1 & 1842 & 858 & 22.81 & 616 & 16.37 & 3762 \\
\hline 20.24 & 2100 & 1018 & 23.93 & 658 & 15.47 & 4254 \\
\hline 21.01 & 1994 & 972 & 23.45 & 656 & 15.83 & 4145 \\
\hline 7.14 & 42 & 3 & 16.67 & 3 & 16.67 & 18 \\
\hline 20.73 & 2036 & 975 & 23.42 & 659 & 15.83 & 4163 \\
\hline 31.58 & 1216 & 560 & 18.02 & 631 & 20.3 & 3108 \\
\hline 5.16 & 911 & 468 & 39.46 & 31 & 2.61 & 1186 \\
\hline 20.26 & 2127 & 1028 & 23.94 & 662 & 15.42 & 4294 \\
\hline 11.07 & 1093 & 495 & 33.4 & 146 & 9.85 & 1482 \\
\hline 29.98 & 1034 & 533 & 18.95 & 516 & 18.35 & 2812 \\
\hline 20.26 & 2127 & 1028 & 23.94 & 662 & 15.42 & 4294 \\
\hline 18.35 & 1946 & 1001 & 25.03 & 547 & 13.68 & 3999 \\
\hline 40.88 & 181 & 27 & 9.15 & 115 & 38.98 & 295 \\
\hline 20.26 & 2127 & 1028 & 23.94 & 662 & 15.42 & 4294 \\
\hline 17.25 & 1913 & 974 & 25.65 & 482 & 12.69 & 3797 \\
\hline 47.87 & 211 & 54 & 10.87 & 180 & 36.22 & 497 \\
\hline 20.29 & 2124 & 1028 & 23.94 & 662 & 15.42 & 4294 \\
\hline 18.74 & 1990 & 972 & 25.54 & 503 & 13.22 & 3806 \\
\hline 42.22 & 135 & 56 & 11.48 & 159 & 32.58 & 488 \\
\hline 20.24 & 2125 & 1028 & 23.94 & 662 & 15.42 & 4294 \\
\hline
\end{tabular}


Description of data: Distribution of the control variables, Nigeria

\begin{tabular}{|c|c|c|c|c|c|c|}
\hline \multirow[t]{2}{*}{ Nigeria } & & \multicolumn{5}{|l|}{2003} \\
\hline & & Poorest & $\%$ & Richest & $\%$ & All women \\
\hline \multirow[t]{3}{*}{ Family planning info: Health facility } & No & 153 & 19.64 & 297 & 29.66 & 1135 \\
\hline & Yes & 51 & 19.64 & 176 & 29.54 & 469 \\
\hline & Total & 204 & 19.64 & 473 & 29.42 & 1604 \\
\hline \multirow[t]{3}{*}{ Family planning worker visit } & No & 707 & 19.64 & 659 & 29.3 & 3476 \\
\hline & Yes & 20 & 19.64 & 84 & 37.84 & 222 \\
\hline & Total & 727 & 19.64 & 743 & 20.09 & 3698 \\
\hline \multirow[t]{3}{*}{ FP Info: TV } & No & 703 & 19.64 & 370 & 12.29 & 3011 \\
\hline & Yes & 23 & 19.64 & 373 & 54.37 & 686 \\
\hline & Total & 726 & 19.64 & 743 & 20.1 & 3697 \\
\hline \multirow[t]{3}{*}{ FP Info: Print } & No & 709 & 21.29 & 554 & 16.64 & 3330 \\
\hline & Yes & 18 & 4.96 & 188 & 51.79 & 363 \\
\hline & Total & 727 & 19.69 & 742 & 20.09 & 3693 \\
\hline \multirow[t]{3}{*}{ Modern contraceptive } & No & 27 & 14.59 & 65 & 35.14 & 185 \\
\hline & Yes & 34 & 9.26 & 161 & 43.87 & 367 \\
\hline & Total & 61 & 11.05 & 226 & 40.94 & 552 \\
\hline \multirow[t]{3}{*}{ Information on pregnancy complication } & No & 185 & 17.19 & 224 & 20.82 & 1076 \\
\hline & Yes & 146 & 10.77 & 480 & 35.4 & 1356 \\
\hline & Total & 331 & 13.61 & 704 & 28.95 & 2432 \\
\hline \multirow[t]{3}{*}{ Unmet need for FP } & No & 609 & 19.67 & 599 & 19.35 & 3096 \\
\hline & Yes & 116 & 19.5 & 144 & 24.2 & 595 \\
\hline & Total & 725 & 19.64 & 743 & 20.13 & 3691 \\
\hline \multirow{3}{*}{$\begin{array}{l}\text { Health worker's assistance during pregnancy } \\
\text { outside a facility }\end{array}$} & No & 622 & 25.99 & 261 & 10.91 & 2393 \\
\hline & Yes & 104 & 8.04 & 481 & 37.17 & 1294 \\
\hline & Total & 726 & 19.69 & 742 & 20.12 & 3687 \\
\hline \multirow[t]{3}{*}{ ANC: nurse assisted } & No & 622 & 25.99 & 261 & 10.91 & 2393 \\
\hline & Yes & 104 & 8.04 & 481 & 37.17 & 1294 \\
\hline & Total & 726 & 19.69 & 742 & 20.12 & 3687 \\
\hline \multirow[t]{3}{*}{ ANC: Govt. hospital } & No & 91 & 11.82 & 254 & 32.99 & 770 \\
\hline & Yes & 245 & 14.64 & 451 & 26.94 & 1674 \\
\hline & Total & 336 & 13.75 & 705 & 28.85 & 2444 \\
\hline \multirow[t]{3}{*}{ ANC: Private hospital } & No & 273 & 15.4 & 450 & 25.38 & 1773 \\
\hline & Yes & 63 & 9.39 & 255 & 38 & 671 \\
\hline & Total & 336 & 13.75 & 705 & 28.85 & 2444 \\
\hline \multirow[t]{3}{*}{ ANC: 1st trimester } & No & 258 & 14.45 & 519 & 29.06 & 1786 \\
\hline & Yes & 73 & 11.53 & 181 & 28.59 & 633 \\
\hline & Total & 331 & 13.68 & 700 & 28.94 & 2419 \\
\hline \multirow[t]{3}{*}{ ANC: +4 tetanus injection } & No & 464 & 28.34 & 110 & 6.72 & 1637 \\
\hline & Yes & 253 & 12.74 & 618 & 31.12 & 1986 \\
\hline & Total & 717 & 19.79 & 728 & 20.09 & 3623 \\
\hline \multirow[t]{3}{*}{ ANC: Home } & No & 306 & 13.36 & 681 & 29.74 & 2290 \\
\hline & Yes & 30 & 19.48 & 24 & 15.58 & 154 \\
\hline & Total & 336 & 13.75 & 705 & 28.85 & 2444 \\
\hline Home delivery & No & 128 & 9.22 & 521 & 37.51 & 1389 \\
\hline & Yes & 600 & 25.96 & 222 & 9.61 & 2311 \\
\hline & Total & 728 & 19.68 & 743 & 20.08 & 3700 \\
\hline Delivery: government health facility & No & 658 & 22.5 & 468 & 16.01 & 2924 \\
\hline & Yes & 70 & 9.02 & 275 & 35.44 & 776 \\
\hline & Total & 728 & 19.68 & 743 & 20.08 & 3700 \\
\hline Delivery: private health facility & No & 671 & 21.67 & 497 & 16.05 & 3097 \\
\hline & Yes & 57 & 9.45 & 246 & 40.8 & 603 \\
\hline & Total & 728 & 19.68 & 743 & 20.08 & 3700 \\
\hline Birth assistance: Doctor & No & 711 & 20.92 & 590 & 17.36 & 3399 \\
\hline & Yes & 15 & 5.21 & 152 & 52.78 & 288 \\
\hline & Total & 726 & 19.69 & 742 & 20.12 & 3687 \\
\hline Caesarean section & No & 720 & 20.08 & 693 & 19.33 & 3586 \\
\hline & Yes & 5 & 7.14 & 38 & 54.29 & 70 \\
\hline & Total & 725 & 19.83 & 731 & 19.99 & 3656 \\
\hline
\end{tabular}


Distribution of the control variables, Nigeria (continued)

\begin{tabular}{|c|c|c|c|c|c|c|c|c|c|}
\hline \multicolumn{5}{|l|}{2008} & \multicolumn{5}{|l|}{2013} \\
\hline Poorest & $\%$ & Richest & $\%$ & All women & Poorest & $\%$ & Richest & $\%$ & All women \\
\hline 353 & 19.43 & 833 & 43.89 & 2726 & 545 & 19.63 & 707 & 5.224 & 3385 \\
\hline 461 & 17.08 & 1643 & 39.25 & 4524 & 728 & 18.14 & 1559 & 12.24 & 6128 \\
\hline 3489 & 15.9 & 3115 & 36.93 & 16677 & 4802 & 17.39 & 2338 & 15.75 & 17612 \\
\hline 3534 & 23.99 & 1689 & 11.47 & 14731 & 4909 & 29.41 & 1570 & 9.41 & 16691 \\
\hline 39 & 1.28 & 1871 & 61.24 & 3055 & 69 & 1.99 & 1511 & 43.49 & 3474 \\
\hline 3573 & 20.09 & 3560 & 20.02 & 17786 & 4978 & 24.69 & 3081 & 15.28 & 20165 \\
\hline 3562 & 21.24 & 2847 & 16.98 & 16769 & 4963 & 25.99 & 2505 & 13.12 & 19095 \\
\hline 124 & 7.46 & 755 & 45.43 & 1662 & 155 & 7.41 & 714 & 34.15 & 2091 \\
\hline 152 & 6.49 & 1127 & 48.1 & 2343 & 236 & 7.45 & 1131 & 35.71 & 3167 \\
\hline 548 & 13.09 & 767 & 18.33 & 4185 & 931 & 22.81 & 485 & 11.88 & 4081 \\
\hline 537 & 8.3 & 2531 & 39.13 & 6468 & 1004 & 10.9 & 2449 & 26.59 & 9211 \\
\hline 1085 & 10.18 & 3298 & 30.96 & 10653 & 1935 & 14.56 & 2934 & 22.07 & 13292 \\
\hline 3306 & 20.98 & 3034 & 19.25 & 15760 & 4609 & 25.65 & 2673 & 14.87 & 17971 \\
\hline 246 & 12.81 & 517 & 26.93 & 1920 & 349 & 16.37 & 401 & 18.81 & 2132 \\
\hline 3552 & 20.09 & 3551 & 20.08 & 17680 & 4958 & 24.66 & 3074 & 15.29 & 20103 \\
\hline 3324 & 25.97 & 1357 & 10.6 & 12799 & 4490 & 33.36 & 954 & 7.09 & 13461 \\
\hline 233 & 4.74 & 2191 & 44.61 & 4912 & 469 & 7.06 & 2127 & 32.04 & 6639 \\
\hline 1068 & 10.07 & 3298 & 31.1 & 10603 & 1920 & 14.45 & 2937 & 22.11 & 13285 \\
\hline 907 & 11.28 & 2082 & 25.89 & 8042 & 1679 & 16.1 & 1847 & 17.71 & 10429 \\
\hline 161 & 6.29 & 1216 & 47.48 & 2561 & 241 & 8.44 & 1090 & 38.17 & 2856 \\
\hline 1068 & 10.07 & 3298 & 31.1 & 10603 & 1920 & 14.45 & 2937 & 22.11 & 13285 \\
\hline 776 & 10.04 & 2376 & 30.73 & 7732 & 1396 & 14.38 & 2030 & 20.9 & 9711 \\
\hline 285 & 10.1 & 935 & 33.13 & 2822 & 547 & 14.78 & 920 & 24.86 & 3700 \\
\hline 1061 & 10.05 & 3311 & 31.37 & 10554 & 1943 & 14.49 & 2950 & 22 & 13411 \\
\hline 2739 & 32.56 & 455 & 5.41 & 8411 & 3278 & 41.97 & 267 & 3.42 & 7811 \\
\hline 808 & 8.8 & 3055 & 33.27 & 9182 & 1670 & 13.72 & 2776 & 22.81 & 12168 \\
\hline 3547 & 20.16 & 3510 & 19.95 & 17593 & 4948 & 24.77 & 3043 & 15.23 & 19979 \\
\hline 962 & 9.95 & 3113 & 32.19 & 9670 & 1860 & 14.5 & 2850 & 22.21 & 12830 \\
\hline 106 & 11.36 & 185 & 19.83 & 933 & 60 & 13.19 & 87 & 19.12 & 455 \\
\hline 1068 & 10.07 & 3298 & 31.1 & 10603 & 1920 & 14.45 & 2937 & 22.11 & 13285 \\
\hline 299 & 4.87 & 2742 & 44.69 & 6135 & 631 & 8.1 & 2470 & 31.69 & 7794 \\
\hline 3280 & 28.07 & 823 & 7.04 & 11684 & 4354 & 35.12 & 616 & 4.97 & 12398 \\
\hline 3579 & 20.09 & 3565 & 20.01 & 17819 & 4985 & 24.69 & 3086 & 15.28 & 20192 \\
\hline 3384 & 23.92 & 2095 & 14.81 & 14149 & 4549 & 30.14 & 1682 & 11.14 & 15092 \\
\hline 195 & 5.31 & 1470 & 40.05 & 3670 & 436 & 8.55 & 1404 & 27.53 & 5100 \\
\hline 3579 & 20.09 & 3565 & 20.01 & 17819 & 4985 & 24.69 & 3086 & 15.28 & 20192 \\
\hline
\end{tabular}




\section{Appendix C4}

Description of data: Concentration curves of use of family planning

Ghana (Years 2003, 2008, 2014)
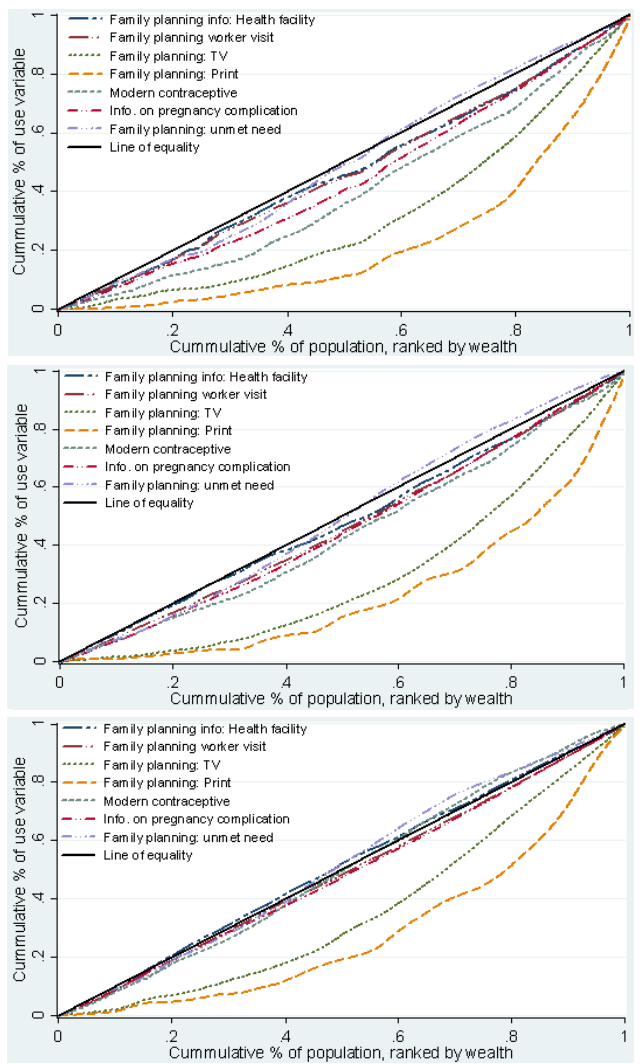

Nigeria (Years 2003, 2008, 2013)
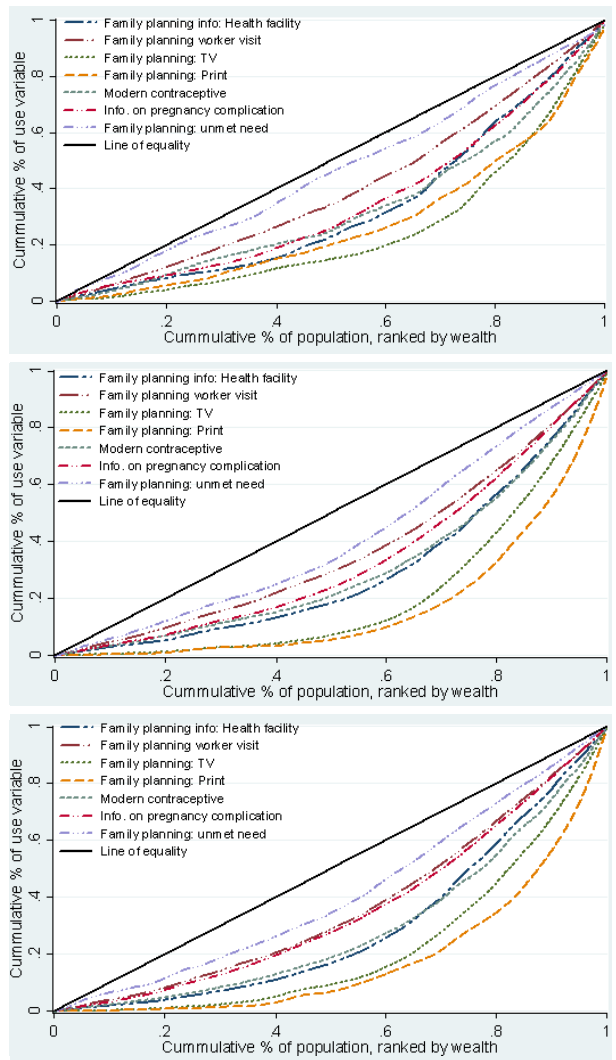


\section{Appendix C5}

Description of data: Concentration curves of use of Antenatal care

Ghana (Years 2003, 2008, 2014)
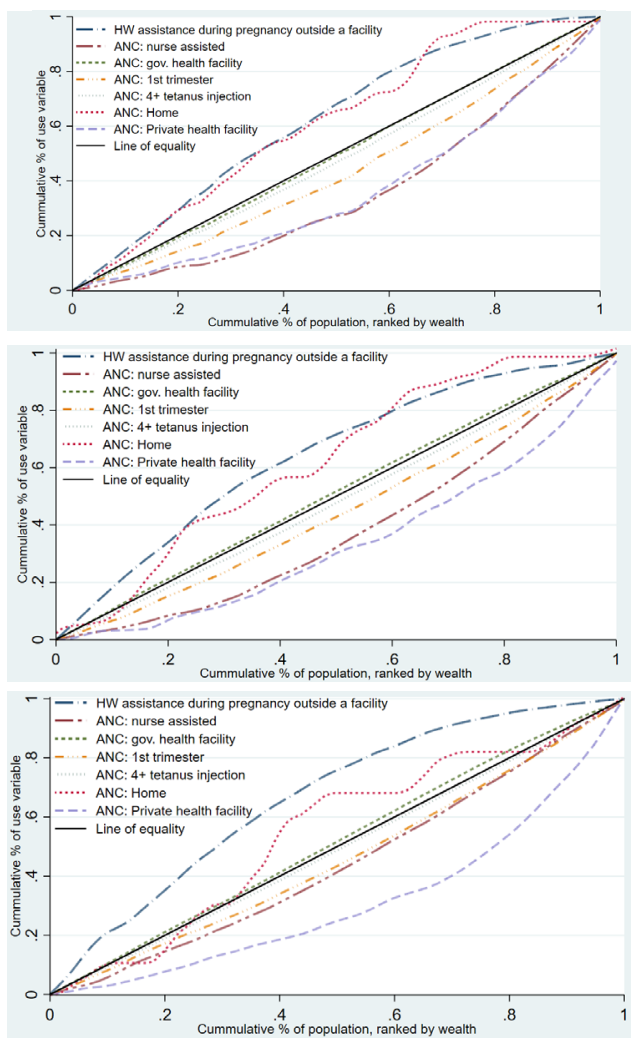

Nigeria (Years 2003, 2008, 2013)
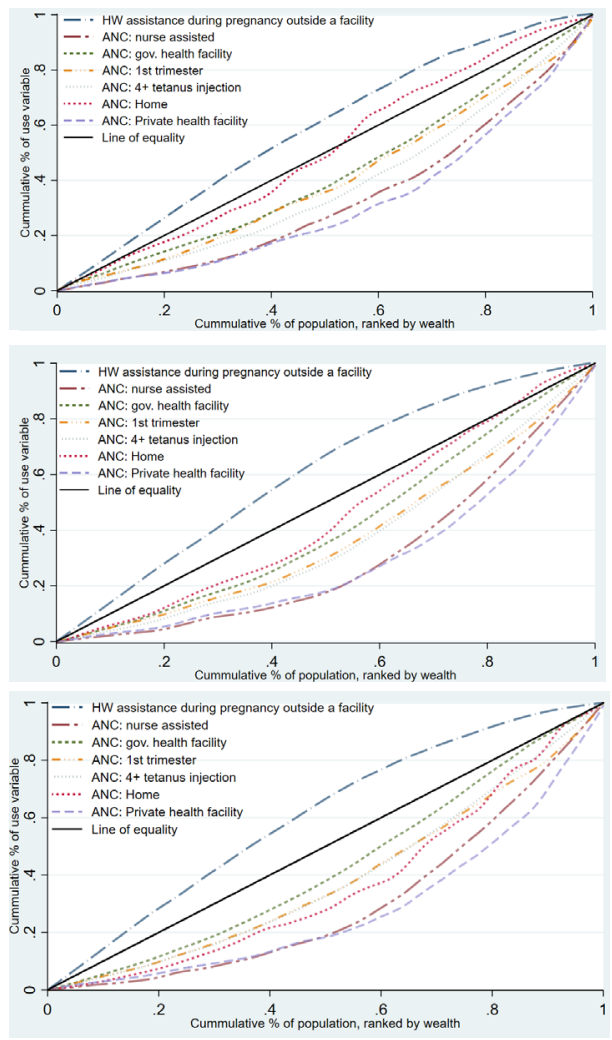


\section{Appendix C6}

Description of data: Concentration curves of use of Delivery care

Ghana (Years 2003, 2008, 2014)
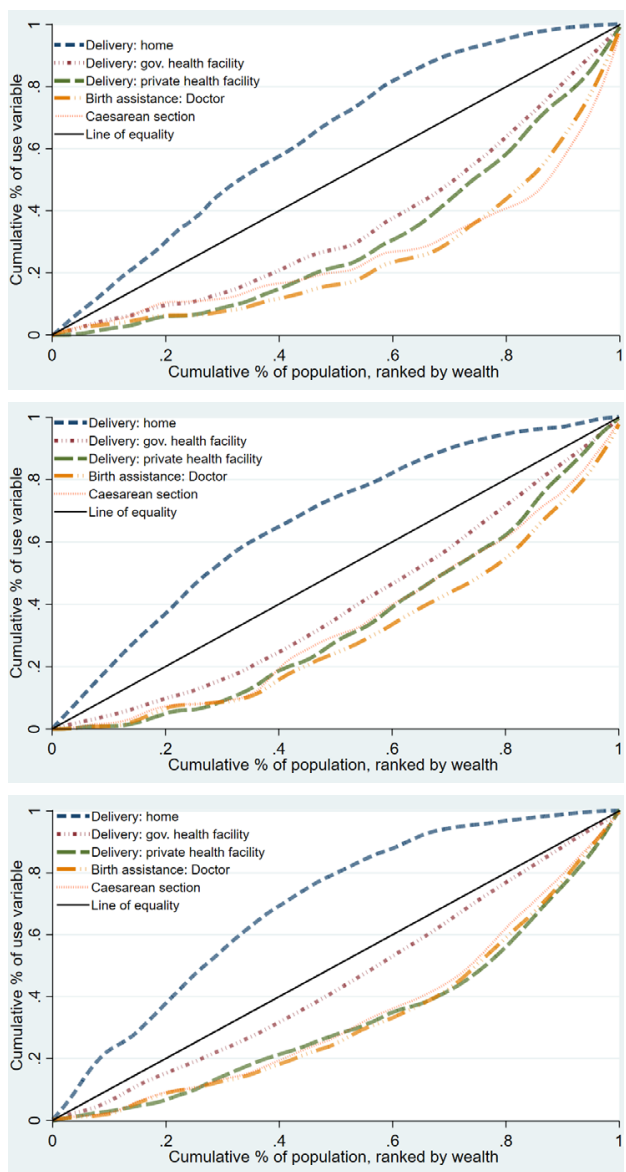

Nigeria (Years 2003, 2008, 2013)
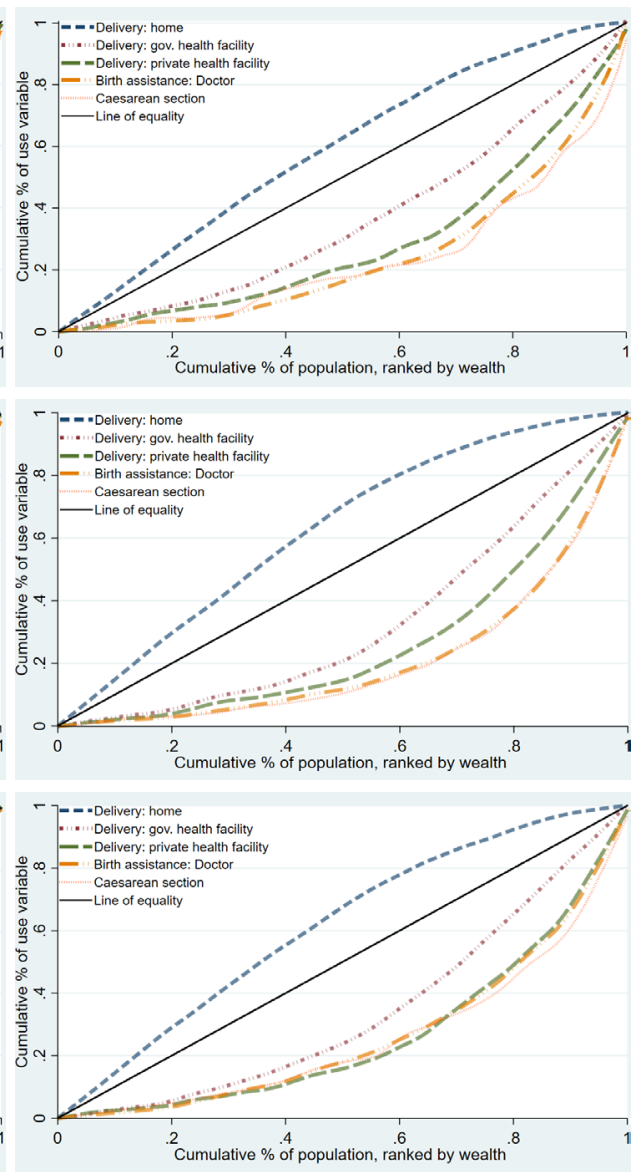


\section{Appendix C7}

Description of data: Concentration Indices of regional Covariates Ghana (years 2000, 2005 \& 2014) and Nigeria (years 2003, 2008 \& 2013)

\begin{tabular}{|c|c|c|c|c|c|c|}
\hline & GHANA & & & NIGERIA & & \\
\hline \multirow[t]{2}{*}{ Service use / covariate } & 2003 & 2008 & 2014 & 2003 & 2008 & 2013 \\
\hline & $\begin{array}{l}\text { concentration } \\
\text { index }\end{array}$ & $\begin{array}{l}\text { concentration } \\
\text { index }\end{array}$ & $\begin{array}{l}\text { concentration } \\
\text { index }\end{array}$ & $\begin{array}{l}\text { concentration } \\
\text { index }\end{array}$ & $\begin{array}{l}\text { concentration } \\
\text { index }\end{array}$ & $\begin{array}{l}\text { concentration } \\
\text { index }\end{array}$ \\
\hline Family planning info: health facility & -0.01 & -0.04 & $-0.04^{*}$ & $0.14^{\star}$ & $0.14^{*}$ & $0.15^{\star}$ \\
\hline Region (Ghana) & -0.01 & -0.04 & $-0.04^{\star}$ & & & \\
\hline Western & -0.09 & 0.06 & -0.07 & & & \\
\hline Central & 0.05 & 0.1 & -0.04 & & & \\
\hline Greater Accra & 0.04 & -0.01 & -0.06 & & & \\
\hline Volta & 0.07 & 0.01 & 0.03 & & & \\
\hline Eastern & -0.05 & -0.15 & -0.03 & & & \\
\hline Ashanti & 0.06 & $0.14^{\star}$ & 0 & & & \\
\hline Brong Ahafo & 0.01 & -0.03 & 0.01 & & & \\
\hline Northern & 0.06 & -0.15 & -0.01 & & & \\
\hline Upper West & -0.05 & 0.01 & 0.02 & & & \\
\hline Upper East & 0.07 & -0.06 & 0.07 & & & \\
\hline F-test & 1.60 & $2.63^{\star}$ & 2.25 & & & \\
\hline \multicolumn{7}{|l|}{ Region (Nigeria) } \\
\hline North Central & & & & 0.11 & 0.07 & $0.13^{*}$ \\
\hline North East & & & & 0.09 & $0.19 *$ & 0.1 \\
\hline North West & & & & $0.39 *$ & $0.29 *$ & $0.24^{*}$ \\
\hline South East & & & & -0.05 & 0.02 & 0.03 \\
\hline South South & & & & 0.1 & 0.02 & 0.05 \\
\hline South West & & & & -0.02 & 0.03 & 0 \\
\hline F-test & & & & $3.21^{\star}$ & $8.64^{*}$ & $22.72^{\star}$ \\
\hline $\begin{array}{l}\text { Family planning worker visit } \\
\text { Region (Ghana) }\end{array}$ & \multicolumn{5}{|c|}{ Region (Ghana) } & $0.4^{*}$ \\
\hline Western & -0.1 & 0.1 & 0 & & & \\
\hline Central & 0 & 0 & 0 & & & \\
\hline Greater Accra & -0.2 & 0 & -0.1 & & & \\
\hline Volta & 0 & 0.1 & -0.1 & & & \\
\hline Eastern & 0 & -0.1 & 0.1 & & & \\
\hline Ashanti & 0.1 & 0.1 & 0.1 & & & \\
\hline Brong Ahafo & -0.1 & 0.3 & 0.1 & & & \\
\hline Northern & 0 & 0 & 0 & & & \\
\hline Upper West & -0.2 & 0 & -0.1 & & & \\
\hline Upper East & 0 & 0 & 0.1 & & & \\
\hline F-test & 1.2 & 1.5 & 1.3 & & & \\
\hline \multicolumn{7}{|l|}{ Region (Nigeria) } \\
\hline North Central & & & & $0.47^{*}$ & $0.36^{*}$ & $0.33^{*}$ \\
\hline North East & & & & 0.3 & $0.34 *$ & $0.3^{*}$ \\
\hline North West & & & & 0.2 & $0.58^{*}$ & $0.37^{\star}$ \\
\hline South East & & & & $0.25^{\star}$ & $0.21^{\star}$ & 0.1 \\
\hline South South & & & & 0.1 & 0 & 0 \\
\hline South West & & & & 0 & $0.16^{\star}$ & $0.08^{\star}$ \\
\hline F-test & & & & $0.84^{*}$ & $22.52 *$ & $11.55^{\star}$ \\
\hline Family planning: TV & $0.37^{\star}$ & $0.4^{\star}$ & $0.28^{*}$ & $0.5^{*}$ & $0.58^{*}$ & $0.56^{\star}$ \\
\hline \multicolumn{7}{|l|}{ Region (Ghana) } \\
\hline Western & $0.22^{*}$ & $0.48^{*}$ & $0.17^{*}$ & & & \\
\hline Central & $0.18^{*}$ & $0.26^{*}$ & $0.22^{*}$ & & & \\
\hline Greater Accra & $0.19 *$ & $0.12^{\star}$ & $0.05^{*}$ & & & \\
\hline Volta & $0.42^{*}$ & $0.38^{*}$ & $0.25^{\star}$ & & & \\
\hline Eastern & $0.23^{*}$ & $0.32 *$ & $0.32^{\star}$ & & & \\
\hline Ashanti & $0.28^{\star}$ & $0.21^{\star}$ & $0.17^{\star}$ & & & \\
\hline Brong Ahafo & $0.28^{\star}$ & $0.44^{*}$ & $0.4^{*}$ & & & \\
\hline Northern & $0.5^{\star}$ & $0.41^{*}$ & $0.37^{\star}$ & & & \\
\hline
\end{tabular}


Concentration Indices of regional Covariates Ghana (years 2000, 2005 \& 2014) and Nigeria (years 2003, 2008 \& 2013) (continued)

\begin{tabular}{|c|c|c|c|c|c|c|}
\hline \multirow[b]{2}{*}{ Service use / covariate } & \multicolumn{3}{|l|}{ GHANA } & \multicolumn{3}{|l|}{ NIGERIA } \\
\hline & 2003 & 2008 & 2014 & 2003 & 2008 & 2013 \\
\hline & $\begin{array}{l}\text { concentration } \\
\text { index }\end{array}$ & $\begin{array}{l}\text { concentration } \\
\text { index }\end{array}$ & $\begin{array}{l}\text { concentration } \\
\text { index }\end{array}$ & $\begin{array}{l}\text { concentration } \\
\text { index }\end{array}$ & $\begin{array}{l}\text { concentration } \\
\text { index }\end{array}$ & $\begin{array}{l}\text { concentration } \\
\text { index }\end{array}$ \\
\hline \multicolumn{7}{|l|}{ Region (Ghana) } \\
\hline Upper West & $0.54^{\star}$ & $0.67^{*}$ & $0.4^{*}$ & & & \\
\hline Upper East & 0.4 & $0.55^{\star}$ & $0.52^{*}$ & & & \\
\hline F-test & $4.71^{*}$ & $6.48^{*}$ & $14.63^{*}$ & & & \\
\hline \multicolumn{7}{|l|}{ Region (Nigeria) } \\
\hline North Central & & & & $0.6^{*}$ & $0.65^{\star}$ & $0.56^{*}$ \\
\hline North East & & & & $0.46^{*}$ & $0.64^{*}$ & $0.69 *$ \\
\hline North West & & & & $0.51^{\star}$ & $0.77^{*}$ & $0.76^{*}$ \\
\hline South East & & & & $0.3^{\star}$ & $0.33^{\star}$ & $0.14^{*}$ \\
\hline South South & & & & $0.29 *$ & $0.33^{*}$ & $0.23 *$ \\
\hline South West & & & & $0.29 *$ & $0.28 *$ & $0.21^{*}$ \\
\hline F-test & & & & $3.84 *$ & $53.47^{*}$ & $85.20 *$ \\
\hline Family planning: Print & $0.54^{\star}$ & $0.52^{\star}$ & $0.42^{\star}$ & $0.43^{*}$ & $0.65^{\star}$ & $0.64^{\star}$ \\
\hline \multicolumn{7}{|l|}{ Region (Ghana) } \\
\hline Western & $0.69 *$ & 0.6 & 0.3 & & & \\
\hline Central & 0.4 & 0.3 & 0.4 & & & \\
\hline Greater Accra & $0.32^{*}$ & $0.47^{\star}$ & 0.1 & & & \\
\hline Volta & 0.4 & $0.57 *$ & $0.48^{*}$ & & & \\
\hline Eastern & 0.5 & 0.4 & 0.3 & & & \\
\hline Ashanti & $0.41^{*}$ & $0.49 *$ & $0.61^{*}$ & & & \\
\hline Brong Ahafo & $0.42^{*}$ & $0.64^{*}$ & $0.55^{\star}$ & & & \\
\hline Northern & 0.3 & 0.4 & 0.9 & & & \\
\hline Upper West & 0.9 & $0.74^{\star}$ & 0.5 & & & \\
\hline Upper East & 0.2 & $0.72^{\star}$ & 0.5 & & & \\
\hline F-test & 0.4 & 0.8 & 1.7 & & & \\
\hline \multicolumn{7}{|l|}{ Region (Nigeria) } \\
\hline North Central & & & & $0.57^{\star}$ & $0.75^{\star}$ & $0.75^{\star}$ \\
\hline North East & & & & $0.37 *$ & $0.61^{*}$ & $0.71^{\star}$ \\
\hline North West & & & & $0.32^{*}$ & $0.77^{\star}$ & $0.77^{*}$ \\
\hline South East & & & & $0.38^{*}$ & $0.45^{*}$ & $0.24^{*}$ \\
\hline South South & & & & $0.25^{*}$ & $0.49 *$ & $0.35^{\star}$ \\
\hline South West & & & & $0.42^{*}$ & $0.41^{*}$ & $0.45^{\star}$ \\
\hline F-test & & & & $1.51^{\star}$ & $12.52^{\star}$ & $19.37^{\star}$ \\
\hline Modern contraceptive & 0 & -0.1 & $-0.06^{\star}$ & 0 & $-0.04^{\star}$ & 0 \\
\hline \multicolumn{7}{|l|}{ Region (Ghana) } \\
\hline Western & 0 & -0.1 & 0 & & & \\
\hline Central & 0.1 & -0.1 & 0 & & & \\
\hline Greater Accra & -0.1 & 0 & $-0.11^{\star}$ & & & \\
\hline Volta & 0 & 0 & 0 & & & \\
\hline Eastern & 0 & 0.1 & 0 & & & \\
\hline Ashanti & 0 & 0 & $-0.09 *$ & & & \\
\hline Brong Ahafo & 0 & 0 & 0 & & & \\
\hline Northern & $0.19 *$ & 0 & 0 & & & \\
\hline Upper West & 0 & 0 & 0 & & & \\
\hline Upper East & 0 & 0 & 0 & & & \\
\hline F-test & 2.1 & 1.2 & $6.69^{*}$ & & & \\
\hline \multicolumn{7}{|l|}{ Region (Nigeria) } \\
\hline North Central & & & & 0 & 0 & 0 \\
\hline North East & & & & -0.1 & 0 & 0 \\
\hline North West & & & & $0.15^{\star}$ & 0 & $0.07 *$ \\
\hline South East & & & & $0.23^{\star}$ & -0.1 & -0.1 \\
\hline South South & & & & 0 & 0 & 0 \\
\hline South West & & & & 0 & 0 & 0 \\
\hline F-test & & & & $10.23^{*}$ & 1 & 2.5 \\
\hline
\end{tabular}


Concentration Indices of regional Covariates Ghana (years 2000, 2005 \& 2014) and Nigeria (years 2003, 2008

\begin{tabular}{|c|c|c|c|c|c|c|}
\hline \multirow[b]{2}{*}{ Service use / covariate } & \multicolumn{3}{|l|}{ GHANA } & \multicolumn{3}{|l|}{ NIGERIA } \\
\hline & 2003 & 2008 & 2014 & 2003 & 2008 & 2013 \\
\hline & concentration & concentration & concentration & concentration & concentration & concentration \\
\hline & index & index & index & index & index & index \\
\hline $\begin{array}{l}\text { Information on pregnancy } \\
\text { complication }\end{array}$ & $0.08^{\star}$ & $0.07^{\star}$ & $0.02^{*}$ & $0.13^{*}$ & $0.12^{*}$ & $0.1^{*}$ \\
\hline \multicolumn{7}{|l|}{ Region (Ghana) } \\
\hline Western & $0.12^{*}$ & $0.09 *$ & 0 & & & \\
\hline Central & 0.1 & 0.1 & 0 & & & \\
\hline Greater Accra & $0.08^{\star}$ & 0 & 0 & & & \\
\hline Volta & 0.1 & 0 & 0 & & & \\
\hline Eastern & $0.09 *$ & 0 & 0.1 & & & \\
\hline Ashanti & 0 & $0.07 *$ & 0.1 & & & \\
\hline Brong Ahafo & $0.06^{*}$ & $0.1^{*}$ & 0 & & & \\
\hline Northern & 0 & 0.1 & 0 & & & \\
\hline Upper West & 0.1 & 0.1 & $0.03^{*}$ & & & \\
\hline Upper East & 0.1 & 0 & -0.1 & & & \\
\hline F-test & 1.7 & $2.48^{*}$ & $3.71^{*}$ & & & \\
\hline \multicolumn{7}{|l|}{ Region (Nigeria) } \\
\hline North Central & & & & $0.11^{*}$ & $0.2^{*}$ & $0.09 *$ \\
\hline North East & & & & 0.1 & $0.07^{*}$ & 0 \\
\hline North West & & & & $0.12^{*}$ & $0.09 *$ & $0.08^{\star}$ \\
\hline South East & & & & $0.17^{*}$ & $0.11^{*}$ & 0 \\
\hline South South & & & & $0.16^{*}$ & $0.07 *$ & 0 \\
\hline South West & & & & 0 & $0.03^{*}$ & $0.05^{\star}$ \\
\hline F-test & & & & $7.17^{\star}$ & $17.87^{\star}$ & $4.13^{\star}$ \\
\hline Family planning: unmet need & 0 & 0 & 0 & 0.1 & $0.18^{*}$ & $0.18^{*}$ \\
\hline \multicolumn{7}{|l|}{ Region (Ghana) } \\
\hline Western & 0 & -0.1 & $-0.17^{\star}$ & & & \\
\hline Central & -0.1 & $-0.1^{*}$ & $-0.12^{\star}$ & & & \\
\hline Greater Accra & $-0.14^{*}$ & -0.1 & -0.1 & & & \\
\hline Volta & 0 & -0.1 & -0.1 & & & \\
\hline Eastern & 0 & 0 & -0.1 & & & \\
\hline Ashanti & -0.1 & -0.1 & $-0.1^{*}$ & & & \\
\hline Brong Ahafo & -0.1 & 0 & $-0.12^{\star}$ & & & \\
\hline Northern & 0 & 0 & 0 & & & \\
\hline Upper West & 0 & 0 & 0.1 & & & \\
\hline Upper East & 0 & -0.1 & 0 & & & \\
\hline F-test & 1.2 & 0.9 & 0.9 & & & \\
\hline \multicolumn{7}{|l|}{ Region (Nigeria) } \\
\hline North Central & & & & 0 & 0 & -0.1 \\
\hline North East & & & & $0.17^{*}$ & 0.1 & 0.1 \\
\hline North West & & & & 0 & $0.21^{*}$ & 0.2 \\
\hline South East & & & & $0.22^{*}$ & 0.1 & $-0.2^{\star}$ \\
\hline South South & & & & -0.1 & 0 & -0.1 \\
\hline South West & & & & 0 & 0 & 0 \\
\hline F-test & & & & $2.27^{\star}$ & $4.22^{\star}$ & $6.36^{\star}$ \\
\hline${ }^{*} p \leq 0.01$ & & & & & & \\
\hline
\end{tabular}

+Professional, technical, managerial, Clerical, or sales 


\section{Appendix C8}

Description of data: Concentration Indices with Covariates Ghana (years 2000, 2005 \& 2014) and Nigeria (years 2003,2008 \& 2013)

\begin{tabular}{|c|c|c|c|c|c|c|}
\hline & GHANA & & & NIGERIA & & \\
\hline \multirow[t]{2}{*}{ Service use / covariate } & 2003 & 2008 & 2014 & 2003 & 2008 & 2013 \\
\hline & $\begin{array}{l}\text { concentration } \\
\text { index }\end{array}$ & $\begin{array}{l}\text { concentration } \\
\text { index }\end{array}$ & $\begin{array}{l}\text { concentration } \\
\text { index }\end{array}$ & $\begin{array}{l}\text { concentration } \\
\text { index }\end{array}$ & $\begin{array}{l}\text { concentration } \\
\text { index }\end{array}$ & $\begin{array}{l}\text { concentration } \\
\text { index }\end{array}$ \\
\hline $\begin{array}{l}\text { Health worker's assistance during } \\
\text { pregnancy outside a facility }\end{array}$ & $-0.25^{\star}$ & $-0.21 *$ & $-0.27 *$ & $-0.17^{\star}$ & $-0.21^{*}$ & $-0.21^{\star}$ \\
\hline \multicolumn{7}{|l|}{ Region (Ghana) } \\
\hline Western & $-0.16^{\star}$ & $-0.29 *$ & $-0.32^{\star}$ & & & \\
\hline Central & -0.08 & $-0.19 *$ & 0.05 & & & \\
\hline Greater Accra & $-0.48^{*}$ & -0.14 & $-0.37^{\star}$ & & & \\
\hline Volta & $-0.14^{\star}$ & -0.02 & -0.07 & & & \\
\hline Eastern & $-0.14^{\star}$ & $-0.15^{\star}$ & $-0.21^{\star}$ & & & \\
\hline Ashanti & $-0.33^{\star}$ & $-0.33^{*}$ & -0.34 & & & \\
\hline Brong Ahafo & $-0.26^{\star}$ & $-0.19 *$ & -0.08 & & & \\
\hline Northern & $-0.05^{\star}$ & -0.1 & $-0.16^{*}$ & & & \\
\hline Upper West & $-0.13^{*}$ & -0.15 & $-0.31^{*}$ & & & \\
\hline Upper East & $-0.12^{\star}$ & $-0.16^{\star}$ & -0.02 & & & \\
\hline F-test & $12.3^{\star}$ & $3.5^{\star}$ & $4.53^{\star}$ & & & \\
\hline \multicolumn{7}{|l|}{ Region (Nigeria) } \\
\hline North Central & & & & $-0.21^{\star}$ & $-0.15^{\star}$ & $-0.19 *$ \\
\hline North East & & & & $-0.08^{*}$ & $-0.08^{*}$ & $-0.11^{\star}$ \\
\hline North West & & & & $-0.08^{*}$ & $-0.06^{\star}$ & $-0.08^{*}$ \\
\hline South East & & & & $-0.65^{\star}$ & $-0.27 *$ & $-0.29 *$ \\
\hline South South & & & & $-0.17^{\star}$ & $-0.21^{\star}$ & $-0.2^{\star}$ \\
\hline South West & & & & $-0.26^{*}$ & $-0.24^{*}$ & $-0.23^{*}$ \\
\hline F-test & & & & $16.92^{\star}$ & $67.68 *$ & $37.12^{\star}$ \\
\hline ANC: nurse assisted & $0.29 *$ & $0.24^{*}$ & $0.11^{\star}$ & $0.33^{*}$ & $0.4^{*}$ & $0.39 *$ \\
\hline \multicolumn{7}{|l|}{ Region (Ghana) } \\
\hline Western & $0.28^{*}$ & $0.21^{\star}$ & $0.08^{*}$ & & & \\
\hline Central & $0.24^{*}$ & $0.26^{*}$ & $0.1^{\star}$ & & & \\
\hline Greater Accra & 0.09 & $0.09 *$ & 0.05 & & & \\
\hline Volta & $0.31^{\star}$ & $0.22^{*}$ & $0.13^{\star}$ & & & \\
\hline Eastern & $0.17^{\star}$ & $0.18^{*}$ & $0.14^{*}$ & & & \\
\hline Ashanti & $0.17^{*}$ & $0.12^{*}$ & 0.01 & & & \\
\hline Brong Ahafo & $0.23^{*}$ & $0.18^{*}$ & $0.09 *$ & & & \\
\hline Northern & $0.31^{*}$ & $0.33^{*}$ & $0.19 *$ & & & \\
\hline Upper West & $0.25^{\star}$ & $0.27^{\star}$ & $0.06^{*}$ & & & \\
\hline Upper East & $0.36^{*}$ & $0.25^{\star}$ & 0.14 & & & \\
\hline$F$-test & $3.67^{*}$ & $5.28 *$ & $6.55^{\star}$ & & & \\
\hline \multicolumn{7}{|l|}{ Region (Nigeria) } \\
\hline North Central & & & & $0.21^{*}$ & $0.28^{*}$ & $0.27^{\star}$ \\
\hline North East & & & & $0.44^{*}$ & $0.53^{\star}$ & $0.5^{\star}$ \\
\hline North West & & & & $0.55^{\star}$ & $0.58^{*}$ & $0.51^{*}$ \\
\hline South East & & & & $0.1^{\star}$ & $0.13^{\star}$ & $0.09 *$ \\
\hline South South & & & & $0.2^{*}$ & $0.2^{*}$ & $0.19 *$ \\
\hline South West & & & & 0.05 & $0.1^{*}$ & $0.07^{*}$ \\
\hline F-test & & & & $12.36^{\star}$ & $83.28^{\star}$ & $106.45^{\star}$ \\
\hline $\begin{array}{l}\text { ANC: government health facility } \\
\text { Region (Ghana) }\end{array}$ & \multicolumn{5}{|c|}{ Region (Ghana) } & $-0.07^{\star}$ \\
\hline Western & -0.02 & -0.01 & -0.02 & & & \\
\hline Central & 0.02 & -0.02 & -0.03 & & & \\
\hline Greater Accra & -0.03 & -0.01 & $-0.07 *$ & & & \\
\hline Volta & -0.01 & -0.03 & 0.02 & & & \\
\hline Eastern & 0.01 & 0.02 & -0.02 & & & \\
\hline Ashanti & -0.02 & $-0.04^{\star}$ & $-0.05^{\star}$ & & & \\
\hline Brong Ahafo & 0.01 & 0.02 & -0.01 & & & \\
\hline
\end{tabular}


Description of data: Concentration Indices with Covariates Ghana (years 2000, 2005 \& 2014) and Nigeria (years 2003, 2008 \& 2013) (continued)

\begin{tabular}{|c|c|c|c|c|c|c|}
\hline & GHANA & & & NIGERIA & & \\
\hline \multirow[t]{2}{*}{ Service use / covariate } & 2003 & 2008 & 2014 & 2003 & 2008 & 2013 \\
\hline & $\begin{array}{l}\text { concentration } \\
\text { index }\end{array}$ & $\begin{array}{l}\text { concentration } \\
\text { index }\end{array}$ & $\begin{array}{l}\text { concentration } \\
\text { index }\end{array}$ & $\begin{array}{l}\text { concentration } \\
\text { index }\end{array}$ & $\begin{array}{l}\text { concentration } \\
\text { index }\end{array}$ & $\begin{array}{l}\text { concentration } \\
\text { index }\end{array}$ \\
\hline \multicolumn{7}{|l|}{ Region (Ghana) } \\
\hline Northern & -0.01 & -0.02 & 0 & & & \\
\hline Upper West & 0.01 & 0 & -0.02 & & & \\
\hline Upper East & 0.04 & -0.01 & 0 & & & \\
\hline F-test & $2.61^{*}$ & $4.95^{\star}$ & $8.66^{*}$ & & & \\
\hline \multicolumn{7}{|l|}{ Region (Nigeria) } \\
\hline North Central & & & & -0.04 & 0.03 & -0.02 \\
\hline North East & & & & -0.01 & 0.01 & 0 \\
\hline North West & & & & 0.01 & $-0.02^{\star}$ & 0 \\
\hline South East & & & & -0.12 & -0.05 & $-0.11^{*}$ \\
\hline South South & & & & 0.05 & 0.01 & -0.02 \\
\hline South West & & & & $-0.11^{*}$ & $-0.08^{*}$ & $-0.08^{*}$ \\
\hline$F$-test & & & & $4.82^{\star}$ & $19.02^{*}$ & $28.48^{*}$ \\
\hline ANC: Private health facility & $0.24 *$ & $0.3^{\star}$ & $0.36^{*}$ & $0.2^{*}$ & $0.23^{\star}$ & $0.27^{\star}$ \\
\hline \multicolumn{7}{|l|}{ Region (Ghana) } \\
\hline Western & 0.22 & 0.18 & 0.14 & & & \\
\hline Central & -0.07 & 0.22 & $0.36^{*}$ & & & \\
\hline Greater Accra & 0.12 & $0.27^{\star}$ & $0.28^{*}$ & & & \\
\hline Volta & 0.3 & 0.27 & -0.16 & & & \\
\hline Eastern & 0.12 & 0 & 0.11 & & & \\
\hline Ashanti & 0.13 & $0.23^{*}$ & $0.33^{*}$ & & & \\
\hline Brong Ahafo & $0.34 *$ & -0.06 & 0.13 & & & \\
\hline Northern & 0.31 & 0.44 & 0.15 & & & \\
\hline Upper West & -0.16 & -0.43 & 0.48 & & & \\
\hline Upper East & -0.19 & 0.56 & 0.71 & & & \\
\hline F-test & 1.39 & 1.97 & 0.9 & & & \\
\hline \multicolumn{7}{|l|}{ Region (Nigeria) } \\
\hline North Central & & & & 0.11 & 0.01 & 0.09 \\
\hline North East & & & & 0.2 & 0.11 & 0.01 \\
\hline North West & & & & 0 & $0.3^{\star}$ & 0.14 \\
\hline South East & & & & $0.13^{*}$ & $0.12^{*}$ & $0.13^{\star}$ \\
\hline South South & & & & 0.21 & $0.27^{\star}$ & $0.22^{*}$ \\
\hline South West & & & & $0.13^{*}$ & $0.14^{\star}$ & $0.11^{\star}$ \\
\hline F-test & & & & $4.15^{\star}$ & $8.98^{*}$ & $3.65^{\star}$ \\
\hline ANC: 1st trimester & $0.09 *$ & $0.09 *$ & $0.07^{*}$ & -0.01 & 0.03 & 0.04 \\
\hline \multicolumn{7}{|l|}{ Region (Ghana) } \\
\hline Western & 0.11 & 0.07 & 0.04 & & & \\
\hline Central & $0.17^{*}$ & 0.04 & $0.08^{*}$ & & & \\
\hline Greater Accra & 0.08 & $0.08^{*}$ & $0.08^{*}$ & & & \\
\hline Volta & 0.04 & 0.02 & 0.05 & & & \\
\hline Eastern & 0.12 & -0.03 & 0.04 & & & \\
\hline Ashanti & $0.09 *$ & $0.09 *$ & $0.07^{*}$ & & & \\
\hline Brong Ahafo & $0.12^{*}$ & 0.04 & $0.07^{\star}$ & & & \\
\hline Northern & 0.13 & $0.18^{*}$ & 0.04 & & & \\
\hline Upper West & -0.05 & 0 & 0.01 & & & \\
\hline Upper East & -0.03 & -0.02 & 0.01 & & & \\
\hline F-test & 1.53 & $3.1^{\star}$ & $3.82^{\star}$ & & & \\
\hline \multicolumn{7}{|l|}{ Region (Nigeria) } \\
\hline North Central & & & & -0.01 & $0.07^{*}$ & -0.01 \\
\hline North East & & & & -0.12 & -0.07 & -0.08 \\
\hline North West & & & & $-0.19 \star$ & $-0.21^{\star}$ & $-0.16^{\star}$ \\
\hline South East & & & & 0.23 & $0.1^{*}$ & $0.07^{*}$ \\
\hline
\end{tabular}


Description of data: Concentration Indices with Covariates Ghana (years 2000, 2005 \& 2014) and Nigeria (years 2003, 2008 \& 2013) (continued)

\begin{tabular}{|c|c|c|c|c|c|c|}
\hline & GHANA & & & NIGERIA & & \\
\hline \multirow[t]{2}{*}{ Service use / covariate } & 2003 & 2008 & 2014 & 2003 & 2008 & 2013 \\
\hline & $\begin{array}{l}\text { concentration } \\
\text { index }\end{array}$ & $\begin{array}{l}\text { concentration } \\
\text { index }\end{array}$ & $\begin{array}{l}\text { concentration } \\
\text { index }\end{array}$ & $\begin{array}{l}\text { concentration } \\
\text { index }\end{array}$ & $\begin{array}{l}\text { concentration } \\
\text { index }\end{array}$ & $\begin{array}{l}\text { concentration } \\
\text { index }\end{array}$ \\
\hline South South & & & & 0.1 & 0.07 & 0.04 \\
\hline South West & & & & 0.00 & $0.1^{*}$ & 0.07 \\
\hline F-test & & & & $6.23^{\star}$ & $39.75^{*}$ & $28.43^{*}$ \\
\hline $\begin{array}{l}\text { ANC: }+4 \text { tetanus injection } \\
\text { Region (Ghana) }\end{array}$ & \multicolumn{5}{|c|}{ Region (Ghana) } & $0.22^{*}$ \\
\hline Western & 0.01 & 0.03 & 0.02 & & & \\
\hline Central & 0.02 & 0.02 & -0.01 & & & \\
\hline Greater Accra & $0.04^{*}$ & $0.05^{\star}$ & 0.01 & & & \\
\hline Volta & $0.08^{*}$ & 0.01 & 0.01 & & & \\
\hline Eastern & 0.00 & 0.03 & 0.02 & & & \\
\hline Ashanti & $0.04^{*}$ & 0.02 & 0.01 & & & \\
\hline Brong Ahafo & $0.04^{*}$ & $0.05^{\star}$ & 0.01 & & & \\
\hline Northern & 0.05 & $0.05^{\star}$ & 0.01 & & & \\
\hline Upper West & 0.03 & 0.00 & 0.01 & & & \\
\hline Upper East & 0.04 & -0.02 & 0.03 & & & \\
\hline F-test & $2.66^{\star}$ & $4.58^{*}$ & 2.17 & & & \\
\hline Region (Nigeria) & & & & $0.23^{*}$ & $0.27^{\star}$ & $0.22^{\star}$ \\
\hline North Central & & & & $0.15^{\star}$ & $0.16^{*}$ & $0.14^{\star}$ \\
\hline North East & & & & $0.24^{*}$ & $0.3^{*}$ & $0.2^{\star}$ \\
\hline North West & & & & $0.43^{*}$ & $0.4^{*}$ & $0.29 *$ \\
\hline South East & & & & $0.04^{*}$ & $0.06^{*}$ & $0.02^{*}$ \\
\hline South South & & & & $0.13^{*}$ & $0.11^{*}$ & $0.07 *$ \\
\hline South West & & & & 0.02 & $0.06^{*}$ & $0.08^{*}$ \\
\hline F-test & & & & $25.79 *$ & $205.75^{*}$ & $121.51^{\star}$ \\
\hline ANC: Home & $-0.26^{\star}$ & $-0.25^{\star}$ & -0.1 & $-0.23^{\star}$ & $-0.15^{\star}$ & 0.05 \\
\hline \multicolumn{7}{|l|}{ Region (Ghana) } \\
\hline Western & -0.29 & & 0.35 & & & \\
\hline Central & -0.74 & & 0.63 & & & \\
\hline Greater Accra & -0.86 & & -0.83 & & & \\
\hline Volta & -0.21 & & -0.27 & & & \\
\hline Eastern & -0.02 & & * & & & \\
\hline Ashanti & 0.06 & & * & & & \\
\hline Brong Ahafo & $-0.59 *$ & & * & & & \\
\hline Northern & -0.04 & & * & & & \\
\hline Upper West & -0.23 & & * & & & \\
\hline Upper East & 0.25 & & * & & & \\
\hline$F$-test & 1.18 & & * & & & \\
\hline \multicolumn{7}{|l|}{ Region (Nigeria) } \\
\hline North Central & & & & 0.08 & $-0.22^{\star}$ & 0.04 \\
\hline North East & & & & 0.03 & $-0.31^{\star}$ & -0.41 \\
\hline North West & & & & -0.48 & -0.08 & -0.14 \\
\hline South East & & & & $-0.54^{\star}$ & $-0.39 *$ & $-0.23^{\star}$ \\
\hline South South & & & & $-0.26^{\star}$ & $-0.39 *$ & $-0.21^{\star}$ \\
\hline South West & & & & -0.17 & -0.12 & -0.07 \\
\hline F-test & & & & $1.93^{\star}$ & $5.23^{\star}$ & 1.13 \\
\hline${ }^{*} p \leq 0.01$ & & & & & & \\
\hline
\end{tabular}




\section{Appendix C9}

Description of data: Concentration Indices with Covariates Ghana (years 2000, 2005 \& 2014) and Nigeria (years 2003,2008 \& 2013)

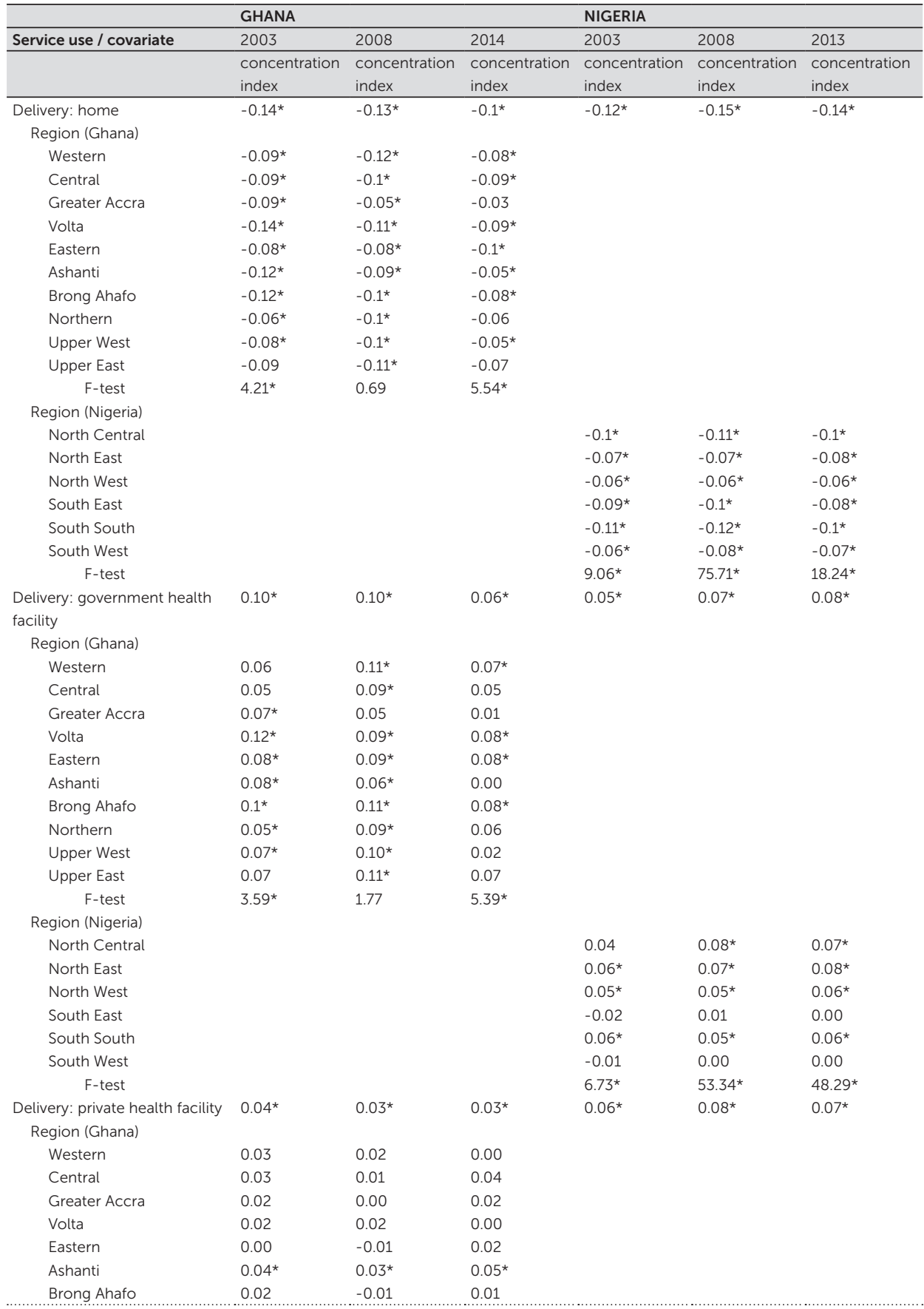


Concentration Indices with Covariates Ghana (years 2000, 2005 \& 2014) and Nigeria (years 2003, 2008 \& 2013) (continued)

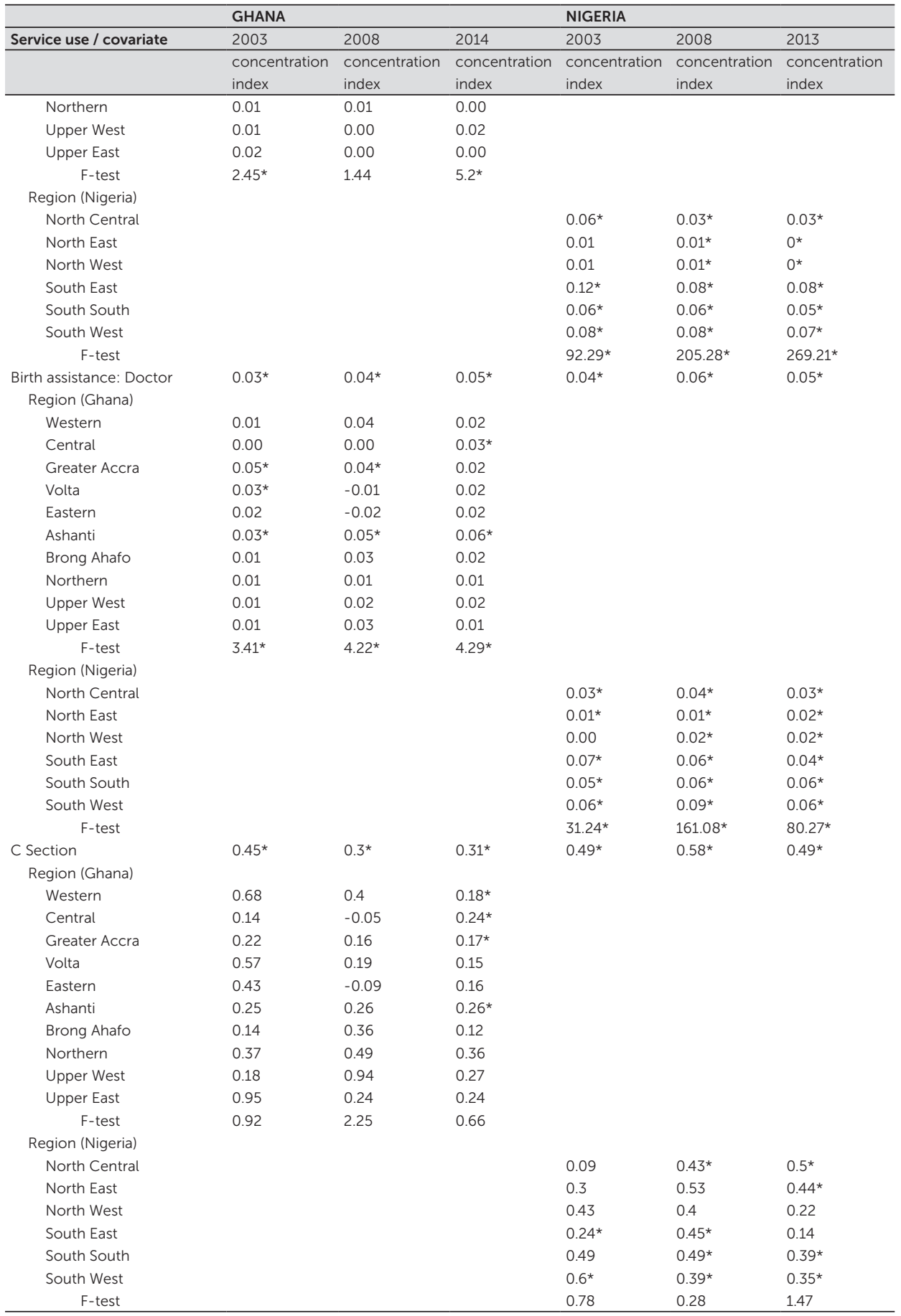

${ }^{*} p \leq 0.01$ 
APPENDIX C - FOR CHAPTER 3 



\section{APPENDIX D}

Additional information for Chapter 5 


\section{Appendix D1}

Description of data: Summary of regional covariates included in the analysis

\begin{tabular}{|c|c|c|c|c|}
\hline \multirow[t]{2}{*}{ Country / Region } & \multicolumn{2}{|c|}{ Low-wealth } & \multicolumn{2}{|c|}{ Higher wealth } \\
\hline & Mean & $\underline{\mathrm{SD}}$ & Mean & $\underline{S D}$ \\
\hline \multicolumn{5}{|l|}{ Burkina Faso } \\
\hline Cascades & 0.01 & 0.10 & 0.05 & 0.21 \\
\hline Centre & 0.01 & 0.11 & 0.12 & 0.32 \\
\hline Centre Est & 0.05 & 0.22 & 0.09 & 0.28 \\
\hline Centre Ouest & 0.08 & 0.27 & 0.08 & 0.27 \\
\hline Centre Sud & 0.04 & 0.20 & 0.05 & 0.22 \\
\hline Est & 0.22 & 0.41 & 0.08 & 0.27 \\
\hline Hauts Basins & 0.06 & 0.23 & 0.12 & 0.33 \\
\hline Nord & 0.06 & 0.23 & 0.08 & 0.27 \\
\hline \multicolumn{5}{|l|}{ Niger } \\
\hline Agadez & 0.02 & 0.14 & 0.01 & 0.12 \\
\hline Diffa & 0.02 & 0.14 & 0.03 & 0.17 \\
\hline Dosso & 0.09 & 0.29 & 0.13 & 0.34 \\
\hline Maradi & 0.21 & 0.41 & 0.22 & 0.41 \\
\hline Tahoua & 0.28 & 0.45 & 0.20 & 0.40 \\
\hline Tillaberi & 0.12 & 0.32 & 0.13 & 0.34 \\
\hline Zinder & 0.25 & 0.44 & 0.19 & 0.40 \\
\hline Niamey & 0.00 & 0.00 & 0.07 & 0.26 \\
\hline \multicolumn{5}{|l|}{ Nigeria } \\
\hline \multicolumn{5}{|l|}{ Ghana } \\
\hline Western & 0.03 & 0.16 & 0.12 & 0.33 \\
\hline Central & 0.02 & 0.13 & 0.13 & 0.34 \\
\hline Volta & 0.02 & 0.12 & 0.20 & 0.40 \\
\hline Eastern & 0.08 & 0.27 & 0.07 & 0.26 \\
\hline Greater Accra & 0.06 & 0.24 & 0.10 & 0.30 \\
\hline Ashanti & 0.05 & 0.21 & 0.21 & 0.41 \\
\hline Brong Ahafo & 0.10 & 0.31 & 0.09 & 0.28 \\
\hline Northern & 0.41 & 0.49 & 0.04 & 0.19 \\
\hline Upper East & 0.16 & 0.36 & 0.01 & 0.11 \\
\hline Upper West & 0.08 & 0.27 & 0.01 & 0.11 \\
\hline \multicolumn{5}{|l|}{ Senegal } \\
\hline Dakar & 0.00 & 0.00 & 0.26 & 0.44 \\
\hline Ziguinchor & 0.01 & 0.10 & 0.04 & 0.19 \\
\hline Diourbel & 0.07 & 0.25 & 0.15 & 0.36 \\
\hline SaintLouis & 0.06 & 0.24 & 0.06 & 0.24 \\
\hline Tambacounda & 0.15 & 0.35 & 0.03 & 0.16 \\
\hline Kaolack & 0.11 & 0.31 & 0.08 & 0.27 \\
\hline This & 0.03 & 0.17 & 0.15 & 0.36 \\
\hline
\end{tabular}




\section{Appendix D2}

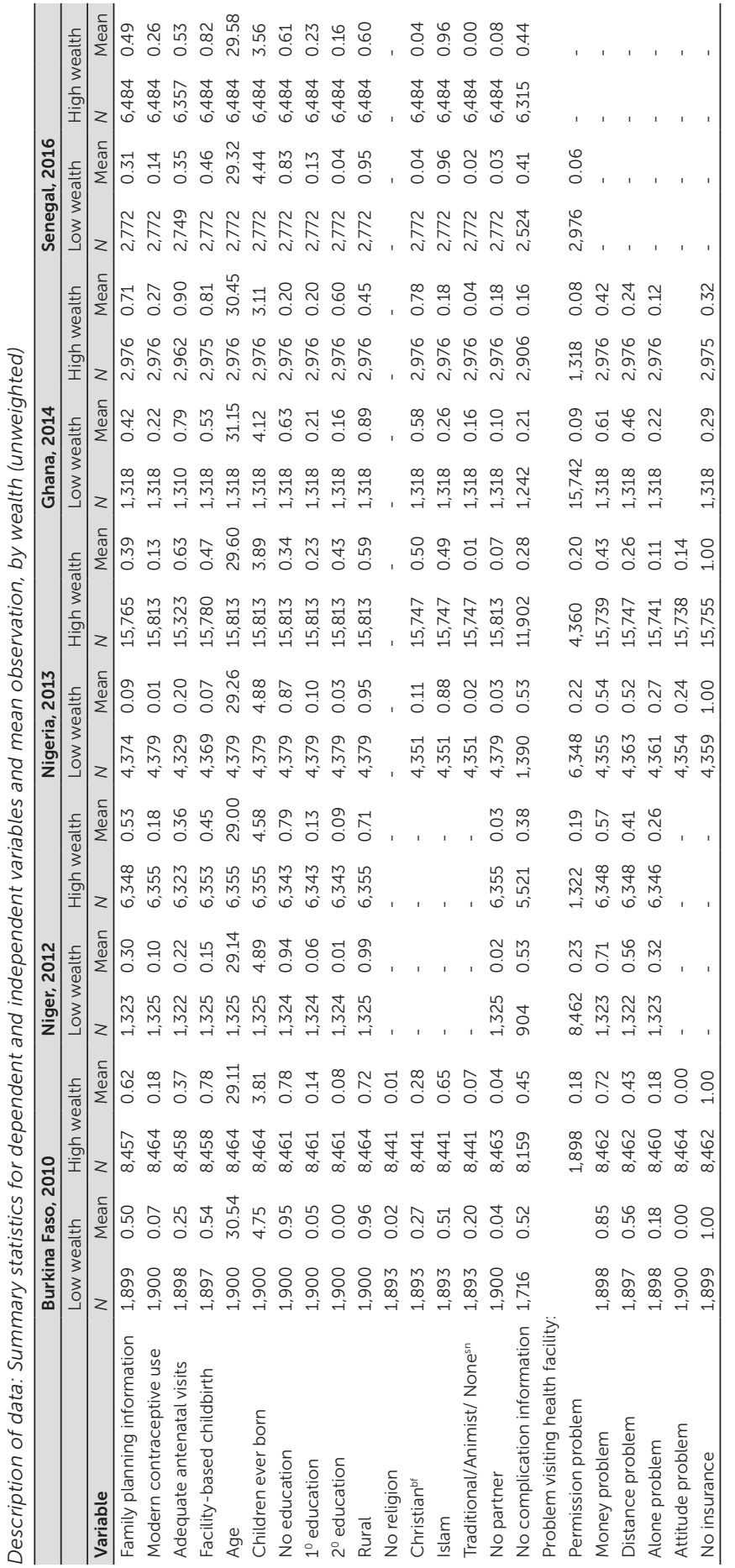


Summary statistics of regional variables and mean observation, by wealth (unweighted)

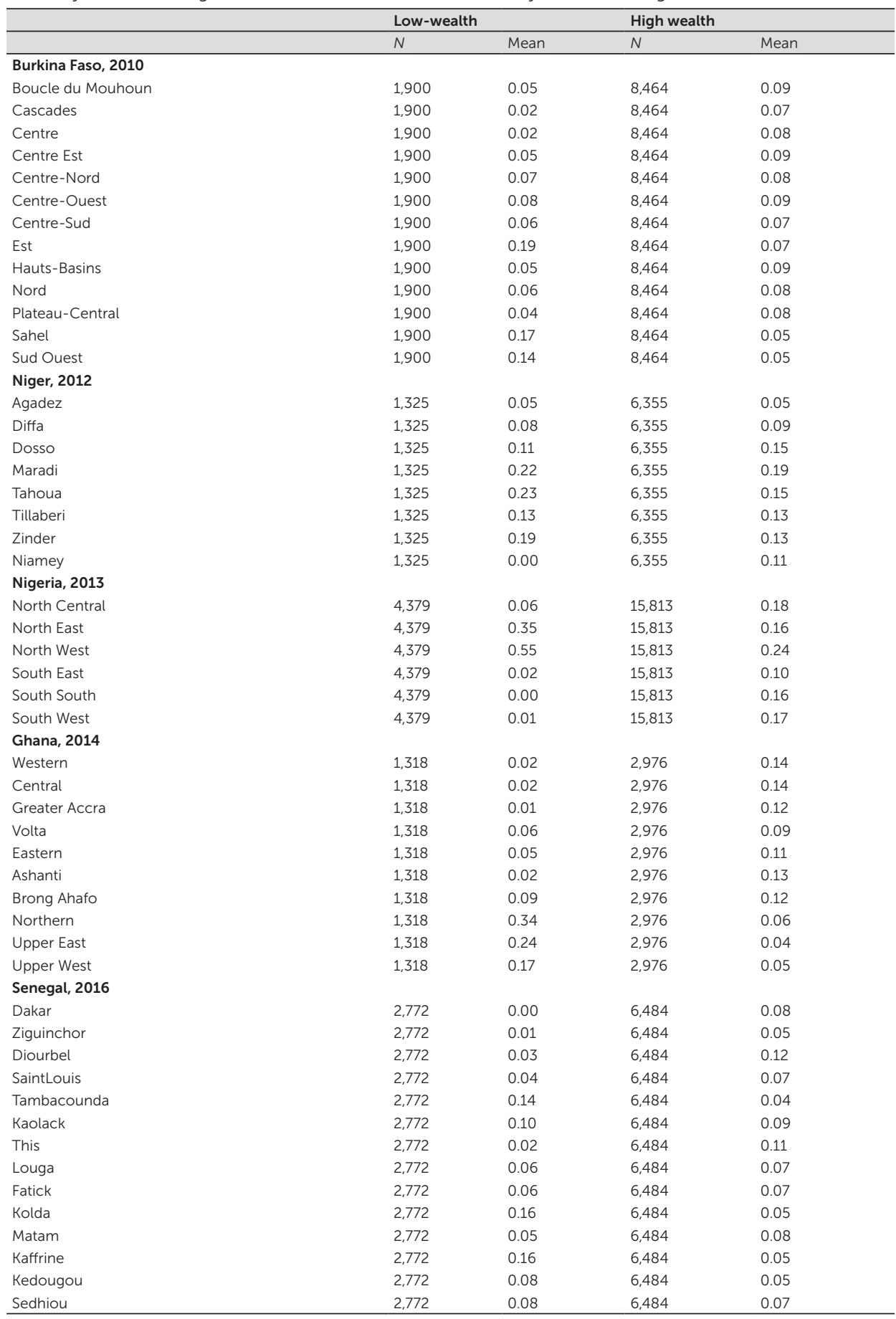


APPENDIX D - FOR CHAPTER 5 

SUMMARY

VALORIZATION ADDENDUM

ACKNOWLEDGMENTS

CURRICULUM VITAE

PUBLICATIONS 
SUMMARY

\section{SUMMARY}


Within- and between-country inequities in health care exist irrespective of the health system and the amount of money spent on it. Countries, including those in the SubSaharan African region, aim to provide equal access to health care services with adequate quality. To narrow inequalities and ensure universal access to sexual and reproductive health, it is imperative to ascertain barriers to equitable reproductive health care and how these vary across different health systems. Evidence on inequalities is scarce in Sub-Saharan African countries and even more so are comparative studies. This may largely be due to the limited capability of developing equity-focused interventions. In Sub-Saharan African countries, most reproductive health care data are limited to the likelihood of service use by sociodemographic or socioeconomic status, thus overlooking differences in reproductive health care services, needs and use across sociodemographic groups. Moreover, the contribution of other demand-side factors, cum socioeconomic status, to inequality in the use of reproductive health care services has scarcely been investigated across Sub-Saharan African countries.

This dissertation analyses the association between socioeconomic disparity and the pattern of reproductive health care services use in Sub-Saharan African countries. The dissertation provides an analyses of the magnitude of wealth-related inequality in the use of reproductive health care services and changes over time, as well as on horizontal inequalities in the determinants of inequalities. The sociodemographic factors that contribute to the observed wealth-based inequalities in reproductive health care services use among women of reproductive age (15-49 years) across SubSaharan Africa countries are identified. Cross-country comparisons further provide evidence on the ways a group position determines service use in diverse health system settings. In view of this, the dissertation fills the knowledge gap on inequalities in the use of reproductive health care services across Sub-Saharan African countries. This dissertation has six chapters summarized below.

Chapter 1 of this dissertation outlines the scope of the dissertation as well as the key concepts. The chapter also provides background information on reproductive health care in Sub-Saharan Africa, the study context and the central aim of the dissertation and datasets used. As stated in the chapter, the purpose of the dissertation is to explore the association between inequalities in the utilization of reproductive health care and society-wide inter-group inequalities among women in Sub-Saharan African countries, using Ghana and Nigeria as case studies. Given this aim, the research questions addressed are: (1) what evidence is there on the inequalities in the utilization of reproductive health care services in Sub-Saharan Africa and what is their association with socioeconomic factors?; (2) what are the patterns of reproductive health care 
service use in Ghana and Nigeria and what are the differences and similarities between the countries?; (3) how did the inequalities in reproductive health service use in Ghana and Nigeria change over time?; and lastly, (4) how do the inequalities in reproductive health service use in Ghana and Nigeria compare to those in other countries in the West Africa region?. The dissertation uses different quantitative methods combining a systematic literature review and meta-analysis, cluster analysis and multinomial regression analysis, concentration curve and concentration index, and Fairlie decomposition technique to answer the research questions. The chapter concludes with an outline of the dissertation.

Chapter 2 presents the results of a meta-analysis on inequalities in reproductive health care services across Sub-Saharan Africa. The review included studies published between January 1, 2008 and June 6, 2019 in peer-reviewed journals. The search was limited to articles in the English language. Three databases, PubMed, POPLINE, and JSTOR, were searched for studies that tested the association of socioeconomic position with access/use of reproductive health care services among women living in any Sub-Saharan African country. The search term consisted of four blocks: "inequalities", "access or use", "reproductive health", and "Sub-Saharan Africa". Each block combined various synonyms related to that block. Studies that were quantitative in nature, measured access or use of reproductive health care service at the country level and used the concentration index as a measure of inequality, were selected for review.

In total, 22 articles were identified and included in our meta-analysis. We find that socioeconomic inequality in the use of reproductive health care services was present to the detriment of poorer women. The overall average inequality in reproductive health care services utilization was much lower compared to some service-specific inequality values, e.g. for family planning services, contraceptive services, skilled antenatal care (ANC) services, other components of ANC, and skilled childbirth services. Service categories with an inequality less than the overall average, included HIV services, four or more ANC visits, and postnatal care services. The use of skilled childbirth services, relative to other reproductive health care service categories, was characterized by the most inequitable service use. The use was in favor of women with better socioeconomic status, usually wealthier households. It appeared from the results that countries in Sub-Saharan Africa have put emphasis on reducing the high rates of maternal mortality by focusing interventions on access to skilled care during childbirth. However, programs implemented have not been successful in eliminating socioeconomic disparity in use. 
This chapter, therefore, concludes that the provision of user fees exemptions should encompass all sexual and reproductive health care services. In this way, the differentials in reproductive health care needs and the ability to overcome the use barriers by women in various socioeconomic groups would be equitably addressed from the supply-side. This means that the promotion of context-specific interventions that are needs-based and address socioeconomic inequalities should be embraced.

Chapter 3 studies the patterns in the use of reproductive health care services in Ghana and Nigeria and the related disparities between social groups. Data from the Ghana and Nigeria Demographic Health Surveys (DHS) of 2014 and 2013 are analyzed in this chapter. DHS data are nationally representative cross-sectional surveys carried out in low- and middle-income countries periodically. A cluster analysis method was applied to group women based on the type of reproductive health care services they used. The resulting clusters were titled based on the quality and adequacy of care used by women in each cluster compared to what is usually provided in government-licensed medical facilities. Specifically, in the poor-access cluster, on average, women reported using less and lower quality care than the care usually provided at government-licensed facilities, and, in the high access cluster, women reported using more and better care. Further, regression analyses provided an understanding of factors associated with the differences between the clusters generated in the cluster analyses.

The cluster analysis of family planning services classified $64.2 \%$ of women in Ghana and $71.5 \%$ in Nigeria to the poor-access cluster. The cluster analyses of maternal health services classified $18.5 \%$ of women in Nigeria and $25.4 \%$ of women in Ghana to the poor-access cluster, including women who did not receive institutionalized maternal care. For both countries, the poor-access cluster had a high proportion of members who had home childbirth and used traditional birth attendants during childbirth. As shown by the cluster analysis, a large proportion of women in Ghana and Nigeria had poor-access to family planning services. Most women did not have access to modern contraceptives. They used traditional birth control methods or did not have the means for needed services. These differences in access to maternal health services in both countries reflected a broader gap between women who accessed ANC at government hospitals, government facilities for childbirth with a physician present, and the lowaccess group of women limited to services such as government health posts without skilled assistance during childbirth, or ANC private vendors. This confirms that among women of reproductive age in Ghana and Nigeria, there is unequal access to reproductive health services. 
The chapter concludes that health initiatives, which seek to stimulate the use of reproductive health services in Ghana and Nigeria, need to take into account the variation in access, as reported in this study, to assure the user-centeredness and overall effectiveness of these programs.

Chapter 4 further investigates how the use of reproductive health care services in Ghana and Nigeria has changed over time and whether policies to reduce inequality in access to reproductive health care services in Ghana and Nigeria were effective. The study in this chapter is based on the analysis of cross-sectional country-level data over a 10-year period obtained from the DHS in Ghana and Nigeria. The chapter presents evidence on the magnitude and trends in wealth-related inequality in the use of reproductive health care services in both countries. It also provides insights into horizontal inequalities by describing the changes in the determinants of inequalities in access to reproductive health care services over the years. The analysis in this chapter used concentration curves and concentration indices to measure the magnitude of socioeconomic-related inequalities and horizontal inequalities in the use of reproductive health care services.

The results indicated overall progress in narrowing disparities in the use of reproductive health care services among women in Ghana and Nigeria. However, the results also confirmed that reproductive health care services remained inequitably distributed and that there were differences in the magnitude of inequality within socioeconomic groups of women. We provided insight into horizontal inequalities by describing the changes in the determinants of inequalities in the use of reproductive health care services over the years. We showed that health worker's assistance during pregnancy outside a facility, ANC at government facilities, and home childbirths were more prevalent among poor women in both Ghana and Nigeria. At the same time, the use of $\mathrm{C}$-section services was less common among poorer women in Ghana and Nigeria. We find that country inequalities persisted over time and horizontal inequalities did not reduce substantially over the years. In particular, over the years, women in poorer households in Nigeria experienced changes in the use of reproductive health care services that were not in their favor. Overall, ANC at private facilities, ANC in government facilities, non-facility formations for ANC, family planning information via mass media became less pro-poor over the years observed.

Based on the results, the chapter concludes that the gains made in reducing inequality in the use of reproductive health care services, are short-lived and erode over time, usually before the poorest population group can benefit. To reduce inequality in 
reproductive health care use, interventions should not only be pro-poor oriented, but they should also be sustainable and user-centered.

Chapter $\mathbf{5}$ focuses on the variation in the use of reproductive health care services due to wealth inequalities and the determinants of this variation in Ghana and Nigeria compared to other countries in the region. Using data from the countrywide DHS in Burkina Faso, Niger, Nigeria, Ghana, and Senegal, we applied the decomposition analysis method to quantify the contribution of sociodemographic characteristics to observed disparities in reproductive health care service use between the poorest women and wealthiest women. We used data provided by women of reproductive age who had given birth during the last 5 years before the survey. To determine the extent to which wealth-based differences in exposure to mass media family planning information, use of modern contraceptives, adequate use of ANC visits, use of facilitybased childbirth services and C-sections are due to differences in the observed respondents' characteristics, a non-linear decomposition technique, called the Fairlie decomposition, was applied. The overall gap in the use of reproductive health care services between women in the low-wealth group and the high-wealth group was observed.

Results showed that differences in characteristics between the poorest and wealthiest women explain at least $24 \%$ of the difference in the use of the services studied. The analysis also showed the contribution of maternal characteristics to the observed gap in the use of reproductive health care services. The number of children a woman has ever had and health care seeking autonomy stood out among other maternal characteristics that increased inequality and explained the observed gap in reproductive health care services use. Moreover, the results for the three maternal health services suggested that not all countries, which have abolished user fees for maternal care completely, have performed similarly in reducing the inequality between wealth groups. Nonetheless, in countries with complete fee exemption policies, namely Ghana, Niger, and Senegal, the between wealth groups gaps in having an adequate number of ANC visits, facility-based childbirth and C-section were smaller.

Overall, the chapter concludes that the probability of the use of reproductive health care services is not parallel to the inequality gap. Population-wide policies are too broad and often benefit the better-off. Interventions, which do not promote fairness in access for most vulnerable groups, usually widen the inequality gaps. Public health interventions are needed to target sociodemographic disparities and health facility seeking problems that disadvantage women in poor households. 
Chapter 6 presents a general discussion of the key findings of this dissertation. The key findings are presented in the form of five statements:

\section{Statement 1: The emphasis on childbirth services across Sub-Saharan African countries pulls attention away from pre-pregnancy, pregnancy, emergency obstetrics, and postpartum services.}

This dissertation showed that skilled childbirth services have the largest wealthbased inequalities in service use compared to other reproductive health care services. Other pre-pregnancy, pregnancy, emergency obstetrics, and postpartum services have less pro-wealthy inequality. The findings further confirmed that in Sub-Saharan African countries, the utilization of skilled childbirth services is higher in case of user fee exemption for facility-based childbirth. In addition, services in the continuum of reproductive health care services, including pre-conception, prenatal and postnatal health care have not been given as much attention. Reproductive health care policies in Sub-Saharan African countries appear uncomprehensive in terms of the proportion of services covered. To address the gaps in reproductive health care services use among women, prioritization of all aspects of reproductive health care services has to be the guiding principle in future policy initiatives. It is also imperative to consider contextual characteristics of the country and target population groups when health innovations are transferred from one to another country, or when research is being translated for policy action.

\section{Statement 2: The wealth-gap in the use of reproductive health care services by women follows a comparable pattern in Sub-Saharan African countries.}

As outlined in this dissertation, the user fee waiver policies for maternal care services alone do not increase equity in the use of reproductive health care services in SubSaharan African countries. While subsidies or removal of user fees for service may increase utilization among pregnant women, the effect on equity is marginal and not consistent across Sub-Saharan African countries. In particular, countries with pro-poor programs, like user fee waivers, still have persistent inequalities in service use. It is important to revise the resources and current user fee policies to reflect and address the needs of the vulnerable population. In addition, inequity in the use of reproductive health care is not limited to socioeconomic or wealth barriers and constraints encountered at the point of service use. Equity should be made a national and state health priority. A comprehensive approach towards equity in health care service at all stages of policy processes is needed to foster the reduction of inequalities in health outcomes and to improve access to quality reproductive health care services across social groups more effectively. 
Statement 3: Wealth-based inequalities in the use of reproductive health care services by women in Sub-Saharan Africa, sustain over time because poor women in this region are not adequately targeted for additional support in population-wide health programs.

As shown in this dissertation, over the years and across socioeconomic groups, most equity indicators of reproductive health care services use in Ghana slightly improved. In Nigeria, equity improvements in the use of reproductive health care services over the years were unfavorable to women in poor households. Thus, poor women in diverse social groups remain at a disadvantage of adequate reproductive health care service use while wealthier women benefit from the services improvements. Countries with national maternal health care policies like Burkina Faso, Ethiopia, Ghana, and South Africa have appreciable narrow inequalities and lower wealth-based gaps, unlike Nigeria and Senegal with limited regional population coverage. The expectation is that new interventions will diffuse to disadvantaged groups after the wealthier population has almost total coverage. However, this dissertation shows that reproductive health care interventions do not progress to the point they eventually benefit the poorer populations absolutely. Where people cannot (are unable to or not allowed to) make use of health services, such policies only benefit advantaged groups, who are able to get to the point of service delivery.

Statement 4: The reproductive health care interventions in Sub-Saharan African countries need to be structured, evidence-driven and adequately monitored to be able to narrow the inequality gap in use of services and sustain the gains made.

As indicated by this dissertation, inequities in the use of reproductive care exist and persist over time. However, countries with longer on-going fee exemption policies have comparable inequality gaps that appear to stall. To reduce inequalities in service use, health care stakeholders need to move away from the single path-dependent fee waivers in reproductive health care and should acknowledge the uncertainties by employing a multi-perspective approach combining the fee waiver policies with other equity-enhancing interventions. Placing an issue on the policy agenda, formulating policies and instruments for appropriate action and then implementing action effectively to improve health status should be evidence-based. Particularly in the Sub-Saharan African region, there are indications that decision-makers do not always adequately use relevant information from research. Across Sub-Saharan countries, there is a strong need for evidence to be generated and translated to inform policymaking, resource allocation and patterns of services, and the use of resources by practitioners in delivering patient care. Decision-makers in this region need to reconsider the traditional decision-making approaches in health care and integrate 
adaptive systems principles when designing new interventions, including reproductive health care interventions.

\section{Statement 5: To be effective, targeted fee exemptions policies for poor women need to address both coverage gaps and equity gaps.}

This dissertation examines both the inequity gap in reproductive health care services use in Sub-Saharan Africa, taking Ghana and Nigeria as case studies, as well as the coverage gap in reproductive health care services in this region. However, as shown in the dissertation, policies in Sub-Saharan Africa have not addressed these gaps throughout the entire continuum of reproductive health care, from pre-pregnancy through post-partum care. Differences in determinants of health prevent women from benefitting from pro-poor reproductive health care interventions and create disparities in access to reproductive health care services across population groups. The gap in the use of available services is as important as the availability of needed health care services. For policies to be effective and leave no one who needs reproductive health care services behind, it is necessary to address both gaps simultaneously. There is a need to monitor both the service availability and related coverage gaps, as well as disparities in service use and related equity gaps. This will help to obtain insights about what reproductive health care program is most equitable and effective and therefore contribute to women's health.

This dissertation has shown that barriers to equitable care vary with health system characteristics, social context, social groups, and type of services. An understanding of the determinants of the variation of inequality in service use is important to narrow the disparity in the use of reproductive health care across Sub-Saharan African countries. 


\section{VALORIZATION ADDENDUM}




\section{Introduction}

Reproductive health in Sub-Saharan Africa requires strong health systems that acknowledge the diverse social contexts and essential services needed. Particularly health systems need to promote equitable use and maximize access to family planning and maternal care services for vulnerable population groups. Macro-level forces, including socioeconomic and political, as well as micro-level factors at the individuallevel, shape the distribution of health and use of these health services. However, due to the complex nature of the health systems, developments take place in isolation rather than synchronously. Because of this, an evidence-based approach is warranted to bridge the divide between macro- and micro-level factors. In the Sub-Saharan African health systems, considerations of equity and access in policy formulations for reproductive health care services are deficient. Therefore, an understanding of the systematic differences between social groups in the use of services, such as that provided in this dissertation, is vital to promote universal access to sexual and reproductive health care services and ensure the wellbeing of women before, during, and after pregnancy.

\section{Target audience}

The reduction in government funding and the introduction of fees for health care services in the '1980s brought with it inequity in the access to quality reproductive health care in Sub-Saharan African countries. Taking Ghana and Nigeria as case studies, this dissertation aims to increase our knowledge and understanding of the determinants of inequalities in reproductive health care in Sub-Saharan Africa. Policymakers and other stakeholders in developmental organizations are the targeted audiences of this dissertation. Women and society are the ultimate beneficiaries.

\section{Result related contents and products}

The evidence from this dissertation shows the inefficiency in the pro-poor policies that aim to achieve long-term objectives through short-term and irregular frameworks. Wealth-based inequalities in the use of reproductive health care services thus sustain or widen. The focus on the provision of a specific reproductive health care service in policies has had detrimental effects on equitable use over time and has reduced coverage of other needed care services. For instance, in one of the case study countries, Ghana, despite user fee exemption policies, there was no significant equity improvement in doctor-assisted births, antenatal care provided at non-facility formations, government and private facilities. For the same indicators in the other case study country, Nigeria, there were instead pro-wealthy equity changes. There were also insignificant equity changes in the magnitude of inequality for unmet needs for 
family planning in both countries.

Though this dissertation notes that the utilization of childbirth services can increase in a favorable health policy environment, like in Ghana, neglect of individual characteristics results in continued inequalities. This is because programs that aim to abate negative outcomes of reproductive health do not pay exclusive attention to the individual-level factors. Access of pregnancy care information, health care coverage in rural areas, the influence of religion or socio-cultural elements, lack of women's autonomy in health facility seeking decision, the need to pay at the health care facility, and distance to health care facilities are such factors. Empirical findings show that shortcomings related to these factors contribute to an increasing disparity in reproductive health care use across all countries on top of wealth differences, regardless of user-fee clauses. This evidence suggests that current user-fees exemption, waiver or subsidies policies increase the use of selected services positively at the cost of other services along the continuum of reproductive health care. Women in Sub-Saharan African countries will benefit from health care systems that are more proactive than reactive by enabling the potential to utilize all services across the continuum of reproductive health care. Moreover, equity frameworks would be useful to guide policy and program activities related to reproductive health in Sub-Saharan African countries. In addition to equity being an ethical notion with no fixed definition, the motivation for reproductive health policies is determined by political agendas, available funds, rather than visions and values. For the reproductive health of women, policy and program activities that seek to increase the lifetime health and decrease sub-group inequality between them should be implemented. Disregard for equity frameworks results in the implementation of sporadic programs that fixates on part/s of reproductive health care that seeks to address maternal mortality. The lack of a structured and consistent approach to reproductive health care reflects the inability to capitalize on failures or achievements, or to account for resource constraints. For these reasons, countries in Sub-Saharan Africa endure ineffective, inefficient, and short-lived gains in lessening inequalities in reproductive health care service provision. The findings of this dissertation indicate that the fixation on wealth barriers and the supply-side factor of a single/selected reproductive health care service, such as childbirth services, creates disparities in the amount and quality of health of different population groups. Women in their reproductive years in Sub-Saharan Africa are using services available along the continuum of care for reproductive health care services, and outside of it that are not necessarily of good quality.

To reduce inequalities in the use of reproductive health care across Sub-Saharan 
African countries, clear public health policies and set agendas that maximizes women's health are crucial. Since polices for reproductive health care tend to increase utilization, although inequitably, the neglect of needs and context appropriateness during the design and implementation creates use gaps. Furthermore, a recognition that reproductive health care interventions are long-term development goals that need to be managed with equally long-term frameworks is crucial to ensure that scarce resources are targeted to where they have the greatest outcome. Based on the evidence that inequalities in Sub-Saharan African countries sustain over time and policies addressing reproductive health issues do not benefit the disadvantaged groups, a bottom-up targeted approach providing an advantage for vulnerable groups would provide equal opportunities for health services use. This can be done by providing opportunities for enrollment (a commitment to a care relationship) of population groups characterized by no or low education, who belong to sales/services/manual occupation groups, who are in low wealth households, or who lack insurance into registries. This should be followed by reproductive health care resource allocation to ensure the adequate supply of services that match needs and can be utilized with little or no cost implication for enrollees nationwide. This strategy can be employed to bridge the divide between macro- and micro-level factors.

\section{Dissemination of products}

All empirical chapters of this Ph.D. dissertation are published in peer-reviewed international journals. Research results have also been presented to peers at academic meetings, at symposiums and published in conference abstracts. Other intended approaches to share relevant research syntheses are targeted messages, face-toface meetings with knowledge users, and engagement with media and participating in researcher/knowledge user networks. Targeted messages from this research will be submitted to stakeholders in health at national and international development organizations, as well as in their funding partners including and not limited to Christian Health Associations in Africa, Islamic Relief, African Union, Economic Community of West African States, UNFPA, USAID, WHO. The author's research interest lies in health evaluation and policy, health equity, social determinants of health, global health agenda setting, and implementation research. He plans to continue research on global and public health issues after this Ph.D. project. 
ACKNOWLEDGEMENTS

\section{ACKNOWLEDGMENTS}


Although this Ph.D. journey started in my mind, I will like to thank the people who helped actualize it. The idea for this book evolved from my employment experience in an NGO in Nigeria, my travels across Africa, and a Global Health master's program. I started this Ph.D. research in 2017 and completed it in 2020. In these past years, I have learned it is easier to turn disappointment into success, than excuses into possibilities. I also learned that positive thinking is not a given but a choice. A debt of gratitude is owed to my family and friends, colleagues, and staff of the Health Services Research department who have contributed to my steadiness over these years.

I thank my supervisors, Professor Milena and Professor Wim. The best team ever! Thank you for your guidance, feedback, and support. Thank you for your trust over the years. I appreciate the guidance and support that goes beyond academia. I must also acknowledge their inspiration individually. Professor Milena, your encouragement to start and continue my Ph.D. despite the obstacles faced - you went above to help me reach my goal. Your tenacity is infectious. From the conversations we had and your own experiences you shared, I could draw from your positive mindset. Professor Wim, for the patience, the support, the freedom that you gave me in my research, and helpful comments, "just a little more", I was able to push my boundaries. I always feel more confident after each meeting. You were a listener who spoke little and did a lot. The best team ever!!

I also thank the members of the assessment committee, Professors Drs. Hans Bosma, Angela Brand, Franziska Gassmann, Philip Baba Adongo, Federica Angeli, for making time to review this book critically and give helpful comments. This helped me to improve the final dissertation greatly.

The Health Services Research department of Maastricht University has been my home for several years and I shared this home with many kind people. I must mention Brigitte, my friend Jo, and Susane. Thank you Suus putting things together behind the scene - organizing meetings and responding to last minute emails. Also Dennis and Svenja. The fond memories of vlaais, soups, and fruits and department outings with you all will remain with me. Thanks to Cho for creating fun - summer and winter. My paranimf, Svenja I say thank you for the time we shared an office and a magic pineapple. Bode and Bimbo for moral support. I cannot forget the kapsalon crew Alex, Anaelle, Jessica, and Lisa. Amazing people!

Iya mi, my dearest mother, Mrs. Mojisola S. Ogundele (MSO). You are gold. I am thankful for your trust and selflessness. You supported my dream of getting a doctorate at 
Maastricht University. Your prayers are being answered! My dear Sisters, Omowumi (Wumzy), and Oluwafemi (Femchi), I thank you for your encouragement, patience, and understanding. You all made sure I never felt alone - selflessly giving time and energy to your younger brother. I still feel your unrestrained love and care. I love you Sisters.

Finally, my wife, Syvetlana, you continuously encourage me and stand by me. You are an inspiration! How you kept me going. I owe you a debt of gratitude for providing sanity when the "curves were not curving", comfort when tests were failing, enduring love when the light at the end of the tunnel was bleak. The future is ours!

This is the Lord's doing; it is marvelous in our eyes. 
ACKNOWLEDGEMENTS 


\section{CURRICULUM VITAE}


Oluwasegun J. Ogundele was born on 20 September 1985 in Ile-Ife, Nigeria. He obtained his bachelor's degree in Demography and Social Statistics at Obafemi Awolowo University, Nigeria in 2010. He later obtained a master's degree in Demography after completing his master's thesis at Southampton University, the United Kingdom in 2013. After this, he was employed as a Consultant Program Officer for the Strengthening HIV/ AIDS Intervention Programs for Most-at-risk Populations at Society for Family Health in Nigeria. In August 2015, he left for the Netherlands to pursue a master's degree in Global Health at Maastricht University. Upon completion, he worked as a teaching assistant for the Faculty of Health, Medicine, and Life Sciences. Covering modules on Global Health Economics, Global Health Policy Analysis, Economic Analysis of Health Systems, Horizontal Trajectory 'Skills', Economics of Healthcare.

Oluwasegun is passionate about improving the health of vulnerable populations, whether that means hands-on fieldwork or in a research capacity. His experience working in Nigeria as a Program Officer for an HIV/AIDS prevention program among female sex workers led him to work on the topic of inequalities in the use of reproductive health care in Sub-Saharan Africa. Since September 2017, Oluwasegun has continued as a Ph.D. researcher at Maastricht University. His research interest includes evidencebased research for population health interventions, health equity, global health agenda setting, and implementation research. 
PUBLICATIONS

\section{PUBLICATIONS}


Ogundele, O. J., Pavlova, M., \& Groot, W. (2018). Examining trends in inequality in the use of reproductive health care services in Ghana and Nigeria. BMC Pregnancy and Childbirth, 18(1), 492. doi:10.1186/s12884-018-2102-9

Ogundele, O. J., Pavlova, M., \& Groot, W. (2020). Inequalities in reproductive health care use in five West-African countries: A decomposition analysis of the wealth-based gaps. International Journal for Equity in Health, 19(1), 44. doi:10.1186/s12939-02001167-7

Ogundele, O. J., Pavlova, M., \& Groot, W. (2020). Patterns of access to reproductive health services in Ghana and Nigeria: results of a cluster analysis. BMC Public Health, 20(1), 549. doi:10.1186/s12889-020-08724-3

Ogundele, O. J., Pavlova, M., \& Groot, W. (2020). Socioeconomic inequalities in reproductive health care services across Sub-Saharan Africa. A systematic review and meta-analysis. Sex Reprod Healthc, 25, 100536. doi:10.1016/j.srhc.2020.100536 
